\title{
Advanced modelling of domain wall dynamics for spintronic devices
}

Author:

Michele Voto
Supervisor:

Prof. Luis LÓPEZ DÍAZ

Co-Supervisor:

Prof. Luis TORRES RINCÓN

A thesis submitted in fulfilment of the requirements

for the degree of Doctor of Philosophy

in the

Doctoral Program on Applied Physics and Technology

November 2, 2017

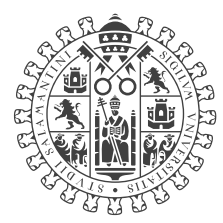

VNIVERSiDAD

BSALAMANCA

CAMPUS DE EXCELENCIA INTERNACIONAL 

D. Luis López Díaz profesor titular de universidad y D. Luis Torres Rincón catedrático de universidad, ambos miembros del Área de Electromagnetismo del Departamento de Física aplicada de la Universidad de Salamanca,

\section{Certifican}

Que la presente Memoria, titulada "Advanced modelling of domain wall dynamics for spintronics devices" ha sido realizada bajo su dirección en el Área de Electromagnetismo del Departamento de Física aplicada de la Universidad de Salamanca por D. Michele Voto, y constituye su Tesis para optar al grado de Doctor en Física.

D. Michele Voto

November 2, 2017

D. Luis López Díaz

D. Luis Torres Rincón

Profesor Titular de Universidad Catedrático de Universidad 



\section{Abstract}

The study of magnetism at the nanoscale has important applications in everyday life. As an example, the vast majority of all data is currently stored in magnetic hard drives, while magnetic sensors are ubiquitous in automotive applications and in the internet of things (IoT) technology. The interaction between the spins of conducting electrons and those of the localized magnetic moments of a ferromagnet is at the base of a new field of studies called Spintronics, whose technological perspectives are to overcome the existing semiconductor technology in terms of power saving, endurance and reliability.

Domain wall propagation is the mechanism through which a magnetic system changes its state when its equilibrium is perturbed via an external action and its dynamics is well described using the micromagnetic formalism. Micromagnetic numerical simulations are a proficient tool that links experimental observations and theoretical predictions, leading the way in the theoretical understanding of magnetization dynamics and domain wall motion.

In chapter 1 we lay down the fundamental physical concepts of the micromagnetic description of magnetism and present the principal analytical tools used throughout the the rest of the work. Chapter 2 is dedicated to the description of the numerical solver used in this work: a custom micromagnetic code based on $\mathrm{C}++$ and CUDA programming languages, developed within the group. Subsequently, we focus on two different problems, making use of the descriptive and predictive power of micromagnetic simulations respectively.

In chapter 3, we investigate the effect of disorder on field driven domain wall dynamics in $\mathrm{CoFeB}$ thin films. Such structures are the building blocks of MRAM memories and the understanding of field driven domain wall dynamics is a key step towards the optimization of device functionality. Exploiting the ability of micromagnetic simulations to reproduce certain disorder features realistically, we get insight into magnetization dynamics taking place at a scale below instruments resolution, uncovering important connections between domain wall dynamics and material disorder features. Disorder triggers internal domain wall dynamics that generates a faster energy dissipation and a faster domain wall propagation in the highfield regime.

In chapter 4, we propose a new spintronic device based on the emission of spin waves by means of the controlled rotation of a domain wall in a ferromagnetic wire. The transmission of information via the periodic oscillatory perturbation of magnetization, called spin wave, offers new perspectives in the design of low power sensors and emitters. We design a system with realistic material characteristics and investigate how the self oscillatory state of a domain wall, induced by the injection of a charge current, can emit a spin wave signal at frequency of tens of $\mathrm{GHz}$ that directly depends on the injected current intensity. 



\section{Acknowledgements}

A doctorate is a long and winding road; looking back, from this end, I see the faces of the many people I met and who accompanied me in this journey. It is a difficult task to fit the gratitude that I feel in these few lines.

To begin with, I think that I have been very lucky to find myself doing something as special as a doctorate school. For that I have to thank my parents, Pasquale and Gabriella, who encouraged me, sustained my choice from the beginning and made me feel loved independently on the outcome of this adventure.

To Gianfranco, I owe my initial discovery of the WALL network and his encouragement in taking a step towards applied physics. I owe him the warm welcome he offered me in my visits at ISI and INRIM, and the pleasure of working together. Importantly, I owe him the calm and friendly attitude with which he managed the project, and his focus on the training of young researchers as the most important goal.

I am especially grateful to Luis, who took me as a student and guided me with care and patience through these years of personal growth. I thank him for the trust and support he has given me from the beginning, for his gentle manners and all the advice he has given me so far. I also thank him for his patience in reading and correcting this thesis.

I'd like to thank Simone, with whom I shared most of this journey, beside the office and several hotel rooms. I am glad that we have been friends rather than colleagues, that we have shared our opinions and discussed about any topics, well beyond physics. I thank him for the collaboration and virtuous competition between us at work, which pushed higher our standards. I would have accomplished much less without him.

My gratitude goes to Luis T., Eduardo, Rocío, Ana, Marcelino, Auxi, Victor, Ricardo and all the Electromagnetism group for the friendly and supportive working environment, for the weekly meetings and for the daily coffees. A special thanks to Eduardo for the many interesting discussions and important advices, and to Luis T., for the guidance, for showing his support and for reading and correcting this thesis.

A big thank you! goes to the ESR fellows of the WALL network with whom I shared this experience, including an amazing Summer School and many meetings, discussions, dinners and drinks all around Europe and beyond.

Thank you to Noel, for all the help and advice in the early stages of this doctorate, thank you to Salvatore for the very special and fun semester we shared at work.

Hard work can be achieved if there is a proper relief outside of it. In Salamanca I found a very special and international group of friends and I can say that part of this thesis has been accomplished thanks to them.

Finally, I thank Cecilia for her love and support, for the peace she brings to me. 



\section{Contents}

$\begin{array}{lr}\text { Introduction } & 1\end{array}$

1 Theoretical background $\mathbf{5}$

1.1 From single spin dynamics to micromagnetics . . . . . . . . . . . 5

1.1.1 Angular momentum and magnetic moment . . . . . . . . 5

1.1.2 Precession of magnetic moment . . . . . . . . . 7

1.1.3 From single spin to continuum approximation . . . . . . . . 7

1.1.4 Phenomenological damping and Landau-Lifshitz-Gilbert equa-

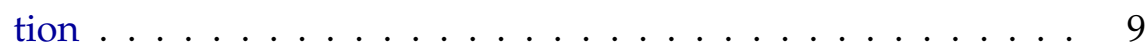

1.2 Free energy and effective field . . . . . . . . . . . . . . 10

1.2.1 Internal free energy and Brown's equations . . . . . . . . . . 11

1.2.2 Exchange interaction . . . . . . . . . . . . . . . . 11

1.2 .3 Anisotropy . . . . . . . . . . . . . . . . 12

1.2.4 Zeeman energy . . . . . . . . . . . . . . . . . . 13

1.2 .5 Magnetostatic energy . . . . . . . . . . . . . . . 13

1.2.6 Dzyaloshinskii-Moriya interaction . . . . . . . . . . . . . . 15

1.3 From nanoparticles to mesoscale magnetism . . . . . . . . . . . . . 17

1.3.1 Monodomains, vortices, multidomains . . . . . . . . . . . 17

1.3.2 Landau and Lifshitz theory of domain walls . . . . . . . . . . 18

1.4 Domain wall dynamics . . . . . . . . . . . . . . . . . . . . 21

1.4.1 Energy minimization and field driven dynamics . . . . . . . . . 21

1.4 .2 One dimensional model . . . . . . . . . . . . . . . 23

2 Numerical simulations $\quad 31$

2.1 Micromagnetic solver . . . . . . . . . . . . . . . . . . . . . . . . . . 31

2.1 .1 Finite differences . . . . . . . . . . . . . . . . 32

2.1.2 Effective field: energy based evaluation . . . . . . . . . . . 32

2.1 .3 Demagnetizing field . . . . . . . . . . . . . . . . . 34

2.1.4 Thermal field . . . . . . . . . . . . . . . . . . . 36

2.1 .5 Workflow .................... . 36

2.1 .6 Time integration . . . . . . . . . . . . . . . 39

2.1 .7 Code verification . . . . . . . . . . . . . . . . 40

2.2 Parallel computing . . . . . . . . . . . . . . . . . . . 41

2.2.1 Hardware architecture . . . . . . . . . . . . . . . . 42

2.2.2 GPU for scientific computing . . . . . . . . . . . . . . . 43 
2.2.3 A parallel computing example: Voronoi tessellation . . . . . . . 44

3 Domain wall dynamics in thin films with disorder 47

3.1 Introduction . . . . . . . . . . . . . . . . 47

3.1 .1 General context . . . . . . . . . . . . . . . . 47

3.1 .2 Motivations . . . . . . . . . . . . . . . . . 48

3.1 .3 The chapter's structure . . . . . . . . . . . . . . . . . . . 49

3.2 Modelling disorder in CoFeB thin films . . . . . . . . . . . 50

3.2 .1 Depinning field . . . . . . . . . . . . . . 50

3.2 .2 Disorder in simulations . . . . . . . . . . . . . . 52

3.2.3 Numerical fit of experimental data . . . . . . . . . . . . . . 54

3.3 Domain wall velocity and energy dissipation . . . . . . . . . . 57

3.3.1 Energy dissipation in ideal and disordered samples . . . . . . . 57

3.3.2 Domain wall velocity and variation of grain diameter . . . . . 60

3.4 Influence of disorder at high fields . . . . . . . . . . . 63

3.4 .1 Gilbert damping and disorder . . . . . . . . . . . 63

3.4.2 Vertical Bloch line dynamics . . . . . . . . . . . . . . . 68

3.4.3 Augmented one dimensional model . . . . . . . . . . . . 73

3.5 Conclusions . . . . . . . . . . . . . . . . . . . . 74

4 Domain wall oscillator as a spin-wave emitter $\quad 77$

4.1 Introduction . . . . . . . . . . . . . . . . . 77

4.2 Theoretical background $\ldots \ldots \ldots$. . . . . . . . . . . . 79

4.2.1 Current induced domain wall motion . . . . . . . . . . . 79

4.2.2 One dimensional theory of pinned domain wall oscillator . . . 82

4.2 .3 Dipolar-exchange spin wave theory . . . . . . . . . . . . . . 84

4.3 Towards spin wave emitter $\ldots \ldots \ldots$. . . . . . . . . 87

4.3.1 Non-uniform current distribution . . . . . . . . . . . 87

4.3.2 Effects of geometrical constriction on domain wall pinned dynamic . . . . . . . . . . . . . . . . . 88

4.3 .3 Out of equilibrium initial condition . . . . . . . . . . . . . . . . . . . . . . . . . . . . . . . .

4.4 Spin wave emission . . . . . . . . . . . . . . . . . . . . . 93

4.4 .1 Specifications . . . . . . . . . . . . . 93

4.4.2 Spin Wave Emission . . . . . . . . . . . . . . . . . . 94

4.4 .3 Application of external field . . . . . . . . . . . . . . . . . 99

4.4.4 Role of dipolar field and unidirectionality . . . . . . . . . . . 101

5 Conclusions and Outlook $\quad 107$

5.1 Domain wall dynamics in disordered systems . . . . . . . . . . . . . . . . . . . . . . .

5.2 Domain Wall-based Spin Wave emitter . . . . . . . . . . . . . . . 110

$\begin{array}{ll}\text { A Voronoi tessellation code } & 113\end{array}$

$\begin{array}{ll}\text { List of publications } & 117\end{array}$ 
Bibliography 



\section{List of Figures}

1.1 Angular momentum and magnetic moment . . . . . . . . . . . . 6

1.2 Classification of materials according to their magnetic properties . . . 8

1.3 Magnetic moment dynamics . . . . . . . . . . . . . . . . . . . . 10

1.4 Interfacial DMI sketch . . . . . . . . . . . . . . . 16

1.5 Energy terms competition: the origin of domains . . . . . . . . . . . 19

1.6 Bloch domain wall in an infinite medium . . . . . . . . . . . . . 20

1.7 head to head domain wall . . . . . . . . . . . . . . 21

1.8 Spherical coordinates . . . . . . . . . . . . . . 23

1.9 One dimensional Bloch wall . . . . . . . . . . . . . . . . . . . . . 24

1.101 D model torques sketch . . . . . . . . . . . . . . . . . . . . 29

1.11 Velocity-field relationship . . . . . . . . . . . . . . . 30

2.1 Runtime operations of micromagnetic code . . . . . . . . . . . . 37

2.2 Workflow of a micromagnetic solver . . . . . . . . . . . 38

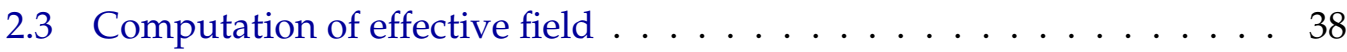

2.4 Initial configuration of standard problem $\# 4 \ldots \ldots$. . . . . . . . . . 41

2.5 Magnetization dynamics in standard problem \#4 . . . . . . . . . . . . 42

2.6 Detail of $\langle\mathbf{m}\rangle_{y}$ evolution in standard problem $\# 4 \ldots \ldots$. . . . . . . 43

2.7 CUDA basic kernel example . . . . . . . . . . . . . . . . . . 45

2.8 Generation of Voronoi tessellation using parallel computing . . . . . . 46

3.1 Creep, depinning, flow regimes . . . . . . . . . . . . . . . 48

3.2 Energy landascape of a thin film . . . . . . . . . . . . . 51

3.3 DW velocity-field curves . . . . . . . . . . . . . . . 52

3.4 Anisotropy constant and easy axis variation over grains . . . . . . . . . 53

3.5 Uniaxial anisotropy easy axis in-plane component distribution . . . . . 54

3.6 Bloch DW separating up and down magnetzed domains . . . . . . . . 55

3.7 DW velocity as a function of the applied field, experiment and simu-

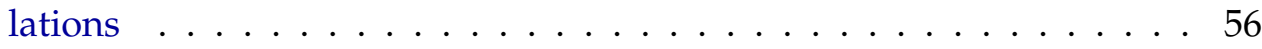

3.8 Time resolved DW dynamics in different regimes . . . . . . . . . . . . 57

3.9 Snapshots of magnetization and dissipated power . . . . . . . . . . 58

3.10 Dissipated power at a critical event . . . . . . . . . . . . . . . . . 59

3.11 DW velocity dependence on grain size . . . . . . . . . . . . . . 60

3.12 Dependence of frequency of critical events with grain size . . . . . . . 61

3.13 High field DW dynamics varying grain size and $\alpha \ldots \ldots$. . . . . . 64 
3.14 Micromagnetic simulations with disorder and 1D model with tilted easy axis . . . . . . . . . . . . . . . 666

3.15 Schematic representation of a vertical Bloch line . . . . . . . . . . 68

3.16 VBL nucleation, propagatio and annihilation . . . . . . . . . . . 69

3.17 Comparison of time-variation of topological charge and instantaneous dissipated power . . . . . . . . . . . . . 70

3.18 DW velocity in micromagnetic simulations and augmented analytical model . . . . . . . . . . . . . . . . . . 75

4.1 Full SW dispersion relation from simulations . . . . . . . . . . . . . 86

4.2 Comparing harmonic and sinc excitation . . . . . . . . . . . 87

4.3 Current distribution in an ellipsoidal particle . . . . . . . . . . . . 89

4.4 Geometrical specifications for depinning threshold study . . . . . . . . 89

4.5 Current distribution for three different notch shapes . . . . . . . . . . . 90

4.6 Nominal and effective depinning current density for different notches and widths . . . . . . . . . . . . . . . . . . . . . . 91

4.7 Snapshots of magnetization in the pinned precession regime . . . . . 92

4.8 External field pulse to trigger precession . . . . . . . . . . . . . 92

4.9 Scheme of the system under study and DW dynamics representation . 94

4.10 Snapshots of magnetization dynamics showing SW emission . . . . . . 95

4.11 Study of DW rotation frequency and SW emission frequency . . . . . . . . 96

4.12 Frequency analysis of DW rotation and SW emission . . . . . . . . . 98

4.13 DW rotation frequency and position as function of effective current density . . . . . . . . . . . . . . . . . . . . 999

4.14 DW rotation and SWs dynamics in the presence of an external field . . 100

4.15 Comparison of the SW excitation caused by a rotating dipolar field and a pinned rotating DW . . . . . . . . . . . . . 102

4.16 Role of non-local stray field in exciting SW . . . . . . . . . . . . 103

4.17 Competition between the in-plane components of exchange and dipolar field is the origin of SW excitation . . . . . . . . . . . . . . 104

4.18 Non-geometrical constriction produces bidirectional SW emission . . . 105

A.1 Generating the 2D tessellation . . . . . . . . . . . . . . . . . 113

A.2 Generate N random seed positions . . . . . . . . . . . . . . . . . 114

A.3 Place seeds at their mesh grid position in 2D space . . . . . . . . . . . 114

A.4 Kernel function that checks the four cells at distance $\pm \mathrm{x}$ Jump, $\pm \mathrm{y}$ Jump from each target cell . . . . . . . . . . . . . . . . . . 115

A.5 Flooding algorithm . . . . . . . . . . . . . . . . . 115

A.6 Jump-flooding algorithm . . . . . . . . . . . . . . . . 116 


\section{List of Tables}

2.1 Butcher tableau of Runge-Kutta methods . . . . . . . . . . . . . . . 40

4.1 Out of plane torques acting on the DW . . . . . . . . . 81

4.2 In plane torques acting on the $\mathrm{DW} \ldots \ldots . \ldots . \ldots 81$ 



\title{
List of Abbreviations
}

\author{
LLG Landau-Lifshitz-Gilbert \\ DMI Dzyaloshinskii-Moriya Interaction \\ SOC Spin Orbit Coupling \\ DW Domain Wall \\ 1D One Dimensional \\ VBL Vertical Bloch Line \\ STT Spin Transfer Torque \\ SW Spin Wave \\ PMA Perpendicular Magnetic Anisotropy \\ RK4 Runge-Kutta solver of order 4 \\ RK5(4)7FC Adaptive time step Runge-Kutta solver of predictor order 5 and corrector 4, \\ 7 steps, First-as-last property, version $\mathbf{C}$ \\ FSAL First Same As Last \\ GPU Graphical Processing Unit \\ CPU Central Processing Unit \\ CUDA Compute Unified Device Architecture
}



Ai miei genitori 



\section{Introduction}

Mankind has been studying magnetism and exploiting its properties for thousands of years, since the lodestones studied by ancient Greeks and the magnetic compasses first used by Chinese sailors. However, the microscopic understanding of magnetism has known a major breakthrough only starting from the late 19th century and throughout the 20th century, following the revolutions of general relativity and quantum mechanics. The quantum mechanical notions of electron's spin and spin dynamics allow us to describe the physics at the atomic scale and explain the origin of magnetism [1]. The magnetization dynamics of a ferromagnetic sample, which consists of a large assembly of atoms, is better described by the micromagnetic theory [2] that examines magnetic materials at a scale small enough to describe local variations of magnetization, while allowing us to use a continuous approximation of magnetization rather than the discrete atomic lattice.

The understanding of magnetism has led to a number of applications that form part of our daily life, well beyond fridge magnets. Magnetic tapes stored information in video and audio cassettes and disks until not so long ago, the vast majority of all data is currently stored in magnetic hard drives, encoded in the magnetization orientation of small regions of magnetic disks. On the other hand, a lot of research is also dedicated to permanent magnets, which are ubiquitous in electrical motors and generators.

The fact that electrons have a spin has additional consequences beyond the generation of an atomic magnetic moment. In a conducting ferromagnet, the localized spins interact with the conduction electrons' spins via the exchange interaction. This effect can lead to the large modification of the resistance of a magnetic spin-valve by changing the magnetization, on one hand, and to the variation of the magnetization orientation by means of a flowing current on the other. Such interaction is at the base of a new field of studies called Spintronics [3], a multidisciplinary research area, where scientists coming from physics, chemistry, materials science, device fabrication and electronics engineering collaborate closely. The technological perspectives of spintronics are to impact on nowadays electronics technology, based on semiconductors devices such as metal-oxide-semiconductor field-effect transistor (MOSFET), with promising applications in new magnetic random-access-memories (MRAM), sensors, emitters and energy harvesting. The most relevant advantage is the natural ability of magnetic systems to preserve their state, which guarantees that no energy is needed to preserve and maintain the state of a device, with subsequent dramatic reduction of energy consumption. 
Domain walls are the interfaces separating two regions (domains) uniformly magnetized along different directions, where the magnetic moments gradually rotate from the orientation of one domain to the next. Domain walls are ubiquitous in magnetism; in fact, their propagation governs magnetization dynamics at the scale relevant for existing technological applications such as MRAM cells [4] and sensors [5] and proposed devices such as racetrack memories [6], shift registers [7] and logic devices [8]. Domain wall motion is, in most cases, the mechanism through which a magnetic system changes its state when its equilibrium is perturbed via an external action. For this reason, domain wall dynamics is deeply linked to the characteristics of the magnetic system, making the study of wall dynamics a common strategy to investigate some of their properties, such as the density of defects [9] and the strength of certain interactions such as Dzyaloshinskii-Moriya interaction (DMI) [10]. Moreover, domain wall dynamics in thin films in the low-field regime belongs to a large class of different physical processes that can be described as the motion of an elastic interface in a weakly disordered medium, and its study can be used to deepen the understanding of critical phenomena of different nature [11,12]

As previously mentioned, magnetization dynamics is well described using the micromagnetic formalism. Micromagnetic numerical simulations are a proficient tool that links experimental observations and theoretical predictions [13], and have led the way in the theoretical understanding of magnetization dynamics and domain wall motion. Recent years' developments in parallel computing, which exploits graphical processing units (GPU) for calculations, allows us to perform simulations with unprecedented computational performances [14], giving access to more intensive systematic simulations of much larger systems.

The objective of the present work is to use advanced micromagnetic simulations to investigate domain wall dynamics in different systems relevant for applications. In chapter 1 we lay down the fundamental physical concepts of the micromagnetic description of magnetism, presenting the principal analytical tools used throughout the the rest of the work. Chapter 2 is dedicated to the description of the numerical solver used in this work. Simulations are performed using a custom micromagnetic code based on $\mathrm{C}++$ and CUDA programming languages, developed within the group throughout the years according to the needs of research. Subsequently, we focus on two different problems, making use of the descriptive and predictive power of micromagnetic simulations, respectively.

The first challenge, studied in chapter 3 , is to investigate the effect of disorder on field driven domain wall dynamics in $\mathrm{CoFeB}$ thin films. $\mathrm{Ta} / \mathrm{CoFeB} / \mathrm{MgO}$ is a common multilayer system, whose properties are widely studied and present in several different experimental devices. Moreover, such structures are the building blocks of MRAM memories and the understanding of field driven domain wall dynamics is a key step towards the optimization of device operation times and functionality. In this sense, an important aspect to consider in domain wall dynamics is the influence 
of the material inhomogeneities and defects in the system, which generate local potential wells that can trap the domain wall, causing the malfunctioning of a device. The generation of a certain amount of defects and material disorder is unavoidable during device fabrication and the complex nature of domain wall propagation in a disordered system cannot be fully described with analytical models. Even though a phenomenological theory that treats the domain wall as an elastic interface moving in a weakly disordered medium exists, which is able to describe domain wall propagation in the low field regime, the effect of disorder on domain wall dynamics at higher fields has not been investigated thoroughly. Exploiting the ability of micromagnetic simulations to reproduce certain disorder features realistically, we are able to get insight into magnetization dynamics taking place at a scale below instruments resolution, uncovering important connections between domain wall dynamics and material disorder features. Disorder triggers internal domain wall dynamics that generates a faster energy dissipation and a faster domain wall propagation in the high-field regime. Importantly, the increment in velocity depends more on disorder features than on the applied field.

The second research aspect, covered in chapter 4 of this work, is the proposal of a new spintronic device based on the emission of spin waves by means of the controlled rotation of a domain wall in a ferromagnetic wire. The transmission of information via the periodic oscillatory perturbation of magnetization, called spin wave, offers new perspectives in the design of low power sensors and emitters. We design a system with realistic material characteristics of $\mathrm{CoFeB}$ and investigate how the self oscillatory state of a domain wall, induced by the injection of a charge current, can emit a spin wave signal at frequency of tens of $\mathrm{GHz}$ that directly depends on the injected current intensity. We perform systematic simulations to investigate the dependence of the emitted signal frequency from the applied current. Moreover, we show how the application of an external field influences such emission by changing the spin waves propagation conditions. We present the requirements for spin wave emission to take place and we describe the conditions through which domain wall pinned rotation is achieved and the mechanism through which spin waves are excited. 



\section{Chapter 1}

\section{Theoretical background}

This first chapter serves as an introduction to the theory of micromagnetics, the formalism used in this thesis. It is inspired by several textbooks $[13,15,1,16,17]$ and it intends to present a brief introduction to the field, while providing the reader with a toolbox of the necessary concepts and expressions common to the more specialized chapters.

\subsection{From single spin dynamics to micromagnetics}

The first step to be taken is the description of the fundamental object of magnetism: the magnetic moment. Its classical definition is intimately linked with angular momentum, providing the basic understanding of the precessional dynamics of magnetic moments. The inclusion of a phenomenological damping, which allows energy dissipation, provides a full description of the observed dynamics, establishing the basic equation that describes magnetization dynamics. Finally, the study of extended mesoscale objects requires the study of the dynamics of a large number of magnetic moments. The passage from a discrete lattice of magnetic moments to a continuous vector field representation of magnetization, provides an effective tool to study magnetization dynamics at the scale we are interested in.

\subsubsection{Angular momentum and magnetic moment}

Let's consider the ideal situation, presented in fig. 1.1, in which a steady current $I$ is flowing along a planar loop immersed in a uniform magnetic field B. Each segment $d \boldsymbol{\ell}$ of the loop will experience a Lorentz force $d \mathbf{F}=I d \boldsymbol{\ell} \times \mathbf{B}$. Due to symmetry, no net force will act on the loop: $\mathbf{F}=I \oint d \boldsymbol{\ell} \times \mathbf{B}=0$. However, the torque on the system will be non-zero if the magnetic field is not perpendicular to the loop plane.

$$
\begin{aligned}
\boldsymbol{\tau} & =\oint \mathbf{r} \times d \mathbf{F}=I \oint \mathbf{r} \times(d \boldsymbol{\ell} \times \mathbf{B}) \\
& =\frac{I}{2}(\oint \mathbf{r} \times d \boldsymbol{\ell}) \times \mathbf{B}+\frac{I}{2} \oint d(\mathbf{r} \times(\overrightarrow{\mathbf{r} \times \mathbf{B}))} 0 \\
& =I A \hat{\mathbf{n}} \times \mathbf{B} .
\end{aligned}
$$




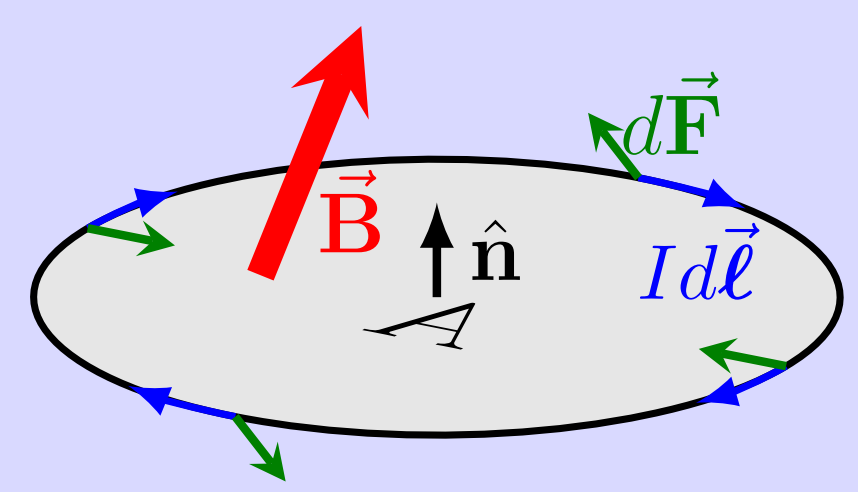

FIGURE 1.1: Schematic representation of the Lorentz's force acting on a loop of electric current in presence of a magnetic field.

Here, $A$ is the surface enclosed by the loop and $\hat{\mathbf{n}}$ is the vector normal to the surface. The vector quantity $\boldsymbol{\mu}=I A \hat{\mathbf{n}}$ is called the magnetic moment and has units of $\mathrm{A} \mathrm{m}^{2}$. A current loop is due to the motion of one or more electrical charges. Since the charge carriers have mass, the magnetic moment $\boldsymbol{\mu}$ is always associated to its angular momentum. The ratio between the magnitudes of angular momentum $L$ and magnetic moment of a given system is called gyromagnetic ratio and denoted with $\gamma$ :

$$
\boldsymbol{\mu}=\gamma \mathbf{L}
$$

Let's consider a very specific system: a classical model of the hydrogen atom, where an electron $e$ is orbiting around the nucleus' proton. This orbiting electron generates the simplest current $I=\frac{q_{e}}{T}$, where $T=2 \pi r / v$ is the electron's orbital period. The magnetic moment associated to its motion has a magnitude $I A=\frac{q_{e} v r}{2}$. This charged particle also has a mass, and its angular momentum is given by $\mathbf{L}_{e}=$ $\mathbf{r} \times \mathbf{p}=m_{e} r v \hat{\mathbf{n}}$. The electron's orbital magnetic moment, as defined above, is then anti-parallel to the electron orbital angular momentum, since the electron charge is negative. The constant of proportionality between these two quantities is $\gamma=\frac{q_{e}}{2 m_{e}}$

In the ground state, the orbital angular momentum of the orbiting electron is $\mathbf{L}_{e}=\hbar \hat{\mathbf{n}}$, so that the associated magnetic moment intensity is

$$
\mu_{B}=|\gamma| L=\frac{q_{e} \hbar}{2 m_{e}} .
$$

$\mu_{B}$ represents a convenient unit to define the size of magnetic moments at the microscopic level. It is called the Bohr magneton and has a value of $9.274 \times 10^{-24} \mathrm{~A} \mathrm{~m}^{2}$. So far we used classical mechanics to describe the electron's orbital motion and the associated orbital angular momentum $\mathbf{L}$ and magnetic moment $\boldsymbol{\mu}$. However, the electron possesses an intrinsic angular momentum, the spin, which cannot be described classically. The intrinsic magnetic moment associated with the electron spin plays a crucial role in magnetism, since without taking it into consideration there would be no net magnetization (see for example [1] pag. 8). To isolate the intrinsic 
angular momentum of the electron, we consider a free electron, not orbiting around an atom's nucleus. Its spin takes a value of $s= \pm \frac{\hbar}{2}$ when measured along a given direction and the associated magnetic moment has a value of $g \mu_{B}$. The factor $g$, simply called $g$-factor, describes the proportionality between the observed magnetic moment and the angular momentum quantum number times the Bohr magneton, it takes a value of 2.00231 for the free electron. The gyromagnetic ratio of the free electron, relating its intrinsic angular momentum or spin and its magnetic moment is

$$
\gamma_{e}=\frac{g_{e} e}{2 m_{e}}=-\frac{g_{e} \mu_{B}}{\hbar}
$$

whose value is $\left|\gamma_{e}\right|=1.76 \times 10^{11} \mathrm{rad} \mathrm{T}^{-1} \mathrm{~s}^{-1}$.

\subsubsection{Precession of magnetic moment}

The torque $\tau$ on a physical system describes the rate of change of the total angular momentum: $\boldsymbol{\tau}=\frac{\mathrm{d} \mathbf{L}}{\mathrm{d} t}$. On the other hand, we saw that an electric charge current loop gives rise to a magnetic moment $\boldsymbol{\mu}$ and that the torque exerted on the loop by an external magnetic field $\mathbf{B}$ is

$$
\tau=\mu \times \mathbf{B} .
$$

We can substitute $\boldsymbol{\tau}$ with $\frac{\mathrm{d} \mathbf{L}}{\mathrm{d} t}$ and $\frac{\mathrm{d} \mathbf{L}}{\mathrm{d} t}$ with $\frac{\mathrm{d} \boldsymbol{\mu}}{\mathrm{d} t} \gamma^{-1}$ to obtain

$$
\frac{\mathrm{d} \boldsymbol{\mu}}{\mathrm{d} t}=\gamma \boldsymbol{\mu} \times \mathbf{B} .
$$

This is the equation of dynamics for a single magnetic moment in a uniform external field. It tells us that the change in $\boldsymbol{\mu}$ is perpendicular to both $\boldsymbol{\mu}$ and $\mathbf{B}$ and that the modulus of the magnetic moment $|\boldsymbol{\mu}|$ is not modified by $\mathbf{B}$. Applying an external magnetic field at an angle $\theta$ with the magnetic moment $\boldsymbol{\mu}$ will make it precess around the applied field, keeping the angle $\theta$ fixed, at a frequency $f_{L}=|\gamma| B(2 \pi)^{-1}$ called the Larmor frequency. Moreover, it states that a stable equilibrium configuration for a magnetic moment in an external field is achieved only when

$$
\boldsymbol{\mu} \times \mathbf{B}=0,
$$

i.e. when the magnetic moment is aligned with $\mathbf{B}$.

\subsubsection{From single spin to continuum approximation}

A solid is said to be magnetic if there is a net magnetic moment per unit volume in the absence of external field. The magnetization $\mathbf{M}$ is defined as the magnetic moment per unit volume $\mathbf{M}=\sum \boldsymbol{\mu} / V$, where the volume $V$ is large compared to the atomic lattice scale and contains a large amount of magnetic moments, whose average varies smoothly in space, so that $\mathbf{M}$ can be considered as a smooth vector field inside the solid [1]. It is measured in units of $\mathrm{A} \mathrm{m}^{-1}$. 
Depending on the magnetic properties of a material, its magnetization can have different responses to an external magnetic field. From now on we will distinguish between the quantity magnetic field $\mathbf{H}$ that act over a magnetic medium and the magnetic flux density $\mathbf{B}$ in units of Tesla (T). In general, the application of an external field $\mathbf{H}$ influences the organization of magnetic moments inside a medium, which in turn influences the total magnetic flux density, following the relation

$$
\mathbf{B}=\mu_{0}(\mathbf{M}+\mathbf{H}) .
$$

In the vacuum, where no magnetic material is present, the relation reduces to $\mathbf{B}=$ $\mu_{0} \mathbf{H}$, where $\mu_{0}=4 \pi \times 10^{-7} \mathrm{H} \mathrm{m}^{-1}$ is the magnetic permeability of vacuum.

Depending on the magnetic properties of materials, a useful classification is schematically shown in fig. 1.2.

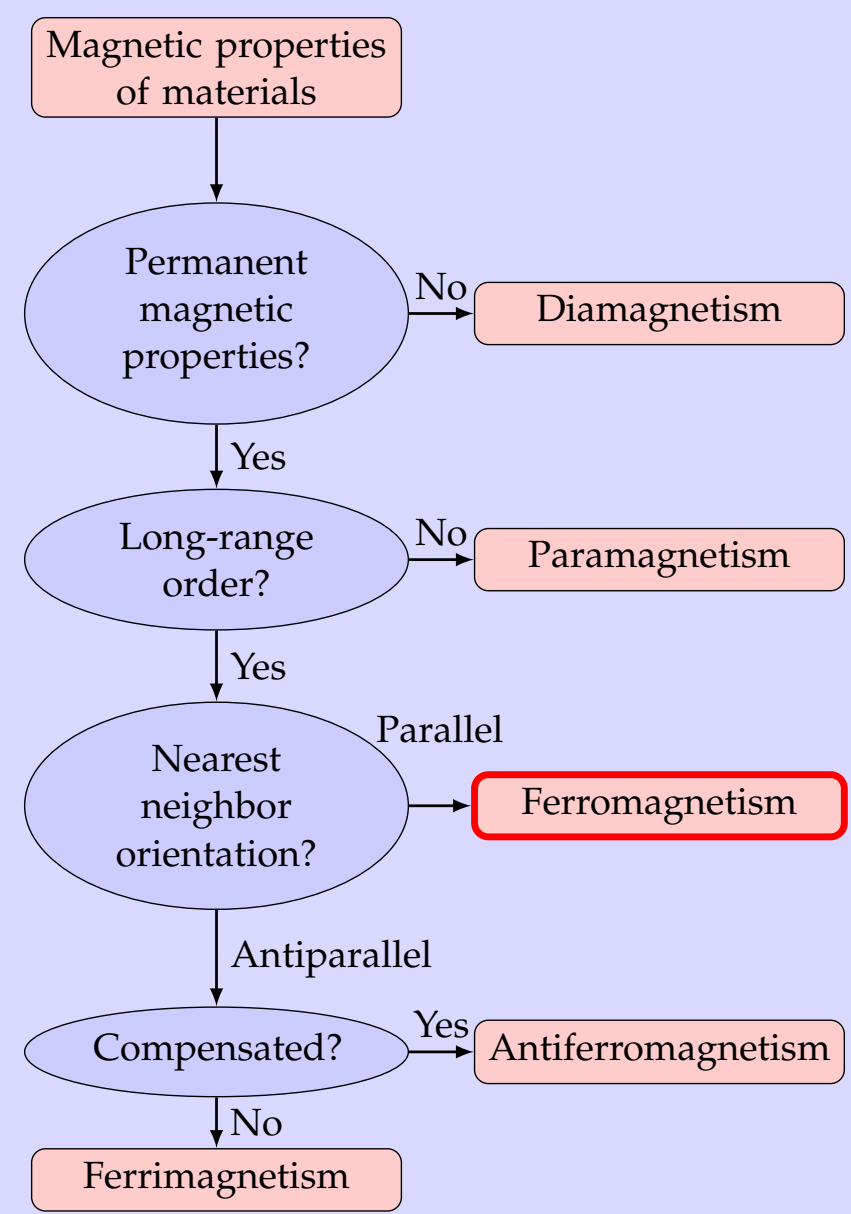

FIGURE 1.2: Classification of materials according to their magnetic properties.

In this work all the magnetic materials considered are ferromagnetic: materials that show a spontaneous magnetization in absence of external fields and whose spins tend to order parallel to each other. The origin of this behaviour will become clear in the next section of this chapter, where we discuss the energy terms that drive 
magnetization dynamics and determine equilibrium states. This behaviour allows us to assume that for every point $\mathbf{r}$ of the sample, in a small volume $V(\mathbf{r})$ all magnetic moments are aligned and it can be defined the saturation magnetization $M_{s}$ as the modulus of the magnetization $|\mathbf{M}(\mathbf{r})|=M_{s} . \quad M_{s}$ is considered constant and dependent on the temperature of the specimen. Assuming constant and uniform temperature in the magnetic system, we can express

$$
\mathbf{M}=M_{s} \mathbf{m},
$$

where $\mathbf{m}$ is the dimensionless normalized magnetization vector. Since we assume a constant modulus for $\mathbf{M}$ and $\mathbf{m}$, the derivatives of these vectors will always be orthogonal to them.

$$
\begin{aligned}
\frac{\partial}{\partial \zeta}(\mathbf{M} \cdot \mathbf{M}) & =\frac{\partial M_{s}^{2}}{\partial \zeta}=0 \\
\frac{\partial}{\partial \zeta}(\mathbf{M} \cdot \mathbf{M}) & =2 \mathbf{M} \cdot \frac{\partial \mathbf{M}}{\partial \zeta} \\
\mathbf{M} \cdot \frac{\partial \mathbf{M}}{\partial \zeta} & =0, \quad \zeta=t, x, y, z .
\end{aligned}
$$

\subsubsection{Phenomenological damping and Landau-Lifshitz-Gilbert equation}

In a ferromagnetic medium each magnetic moment tries to align with a local field, called effective field $\mathbf{H}_{\text {eff }}$, in an attempt to minimise the system's energy. The nature of $\mathbf{H}_{\text {eff }}$ will be described later on. However, in principle, for each spin in our system eq. (1.1) should hold, that is to say, at every point $\mathbf{r}$ :

$$
\frac{\mathrm{d} \mathbf{M}(\mathbf{r})}{\mathrm{d} t}=-\gamma_{0} \mathbf{M}(\mathbf{r}) \times \mathbf{H}_{\mathrm{eff}}(\mathbf{r})
$$

where $\gamma_{0}=|\gamma| \mu_{0}$. This means that magnetic moments would precess indefinitely with a constant angle as in fig. 1.3-a. Experimental evidence, however, tells us that magnetization in samples exposed to an external field tends to align parallel to it, and that magnetic systems evolve towards an equilibrium corresponding to a local energy minimum [16]. To describe the dynamics of this energy minimization process, a phenomenological dissipative term that takes into account a wide range of local dissipative phenomena is added to eq. (1.5). Adding to the effective field a contribution of the form

$$
-\frac{\alpha}{\gamma_{0} M_{s}} \frac{\mathrm{d} \mathbf{M}}{\mathrm{d} t}
$$

in eq (1.5), yields

$$
\frac{\mathrm{d} \mathbf{M}}{\mathrm{d} t}=-\gamma_{0} \mathbf{M} \times \mathbf{H}_{\text {eff }}+\frac{\alpha}{M_{s}} \mathbf{M} \times \frac{\mathrm{d} \mathbf{M}}{\mathrm{d} t},
$$

called Landau-Lifshitz-Gilbert equation (LLG), which prescribes that each spin spirals towards equilibrium as in fig. 1.3-b. This equation is central to the whole thesis. 
It describes accurately magnetization dynamics at a local scale, and its predictive power is proven by the extensive use of micromagnetic simulations in the literature.

Equation (1.6) can be rewritten in explicit form multiplying both sides by $\mathbf{M}$, using simple vector algebra $\mathbf{A} \times(\mathbf{B} \times \mathbf{C})=(\mathbf{A} \cdot \mathbf{C}) \mathbf{B}-(\mathbf{A} \cdot \mathbf{B}) \mathbf{C}$ and the fact that $\mathbf{M} \cdot \frac{\mathrm{d} \mathbf{M}}{\mathrm{d} t}=0$ since the modulus of $\mathbf{M}$ is fixed:

$$
\mathbf{M} \times \frac{\mathrm{d} \mathbf{M}}{\mathrm{d} t}=-\gamma_{0} \mathbf{M} \times\left(\mathbf{M} \times \mathbf{H}_{\mathrm{eff}}\right)-\frac{\alpha}{M_{s}} \frac{\mathrm{d} \mathbf{M}}{\mathrm{d} t} .
$$

Substituting $\mathbf{M} \times \frac{\mathrm{d} \mathbf{M}}{\mathrm{d} t}$ in eq. (1.6) gives the explicit form of LLG equation.

$$
\frac{\mathrm{d} \mathbf{M}}{\mathrm{d} t}=-\frac{\gamma_{0}}{1+\alpha^{2}}\left[\mathbf{M} \times \mathbf{H}_{\mathrm{eff}}+\frac{\alpha}{M_{s}} \mathbf{M} \times\left(\mathbf{M} \times \mathbf{H}_{\mathrm{eff}}\right)\right] .
$$

Equation (1.7) is the basic equation of micromagnetics; in what follows we will discuss the main contributions to the effective field $\mathbf{H}_{\text {eff }}$.
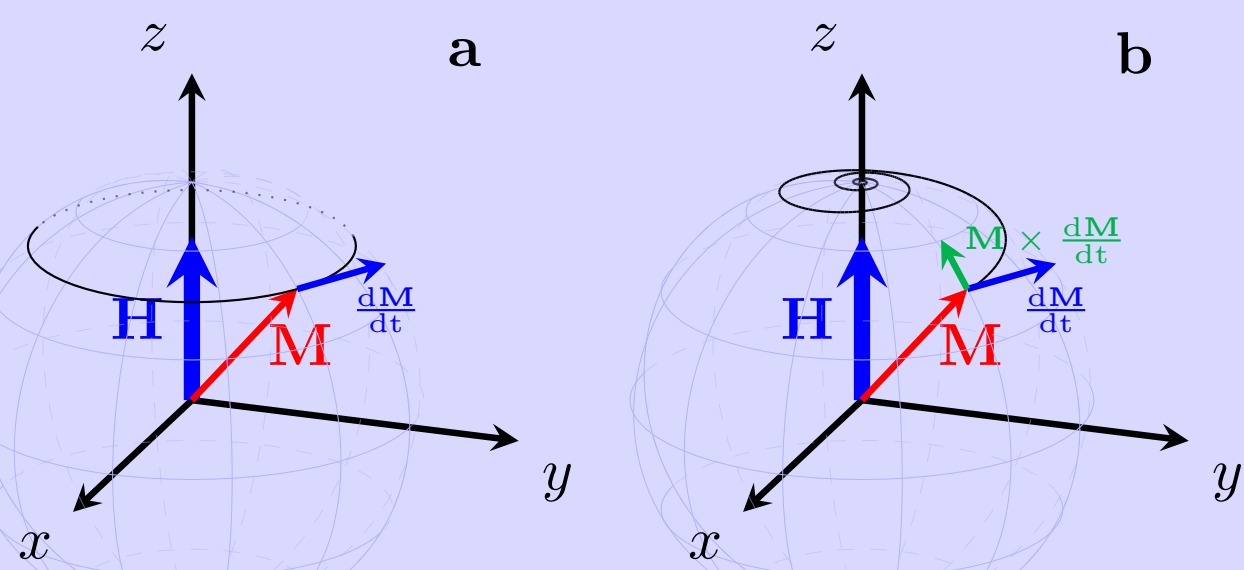

FIGURE 1.3: Magnetic moment dynamics. a A magnetic moment precesses around an effective field with a constant polar angle. b When a dissipative term is introduced, the magnetic moment tends to align to the effective field with a spiraling trajectory, result of the combination of precessional and dissipative dynam-

ics.

\subsection{Free energy and effective field}

As anticipated, magnetization in extended media tends to align to an effective field in order to minimize the internal free energy of the system. In this section, a definition of internal free energy is given and, from that, the effective field is derived. Brown's equilibrium conditions for magnetization are obtained and the most relevant energy terms concurring to the system's free energy are presented. 


\subsubsection{Internal free energy and Brown's equations}

Let's consider a finite sample extending over a region $\Omega$; its internal free energy density $\mathcal{E}$ is a function of the magnetization configuration. It can be shown from variational principles $[2,18]$ that a static equilibrium configuration is obtained when the total free energy is minimized, and this corresponds to

$$
\mathbf{M} \times \frac{\delta \mathcal{E}}{\delta \mathbf{M}}=0
$$

inside the volume, and

$$
\frac{\partial \mathbf{M}}{\partial \mathbf{n}}=0
$$

on the boundary $\partial \Omega$ for the simple case of free boundary conditions. Equations (1.8) and (1.9) are called Brown's equations. They need to be solved in order to find equilibrium configurations of a magnetic system.

The effective field acting on the magnetization is obtained as the functional variation of the energy of the system with respect to the magnetization $[2,18]$ :

$$
\mathbf{H}_{\mathrm{eff}}=-\frac{1}{\mu_{0} M_{s}} \frac{\delta \mathcal{E}}{\delta \mathbf{m}}
$$

we can see that, using eq. (1.10), eq. (1.8) takes the form of the equilibrium condition of a single classical magnetic moment (1.2).

The internal free energy density $\mathcal{E}$ is the sum of different energy contributions that depend on different features of the material and of its magnetization configuration.

\subsubsection{Exchange interaction}

Ferromagnetic materials show spontaneous magnetization above the microscopic scale. This is due to the tendency of magnetic moments in such materials to align parallel to each other, giving rise to magnetic order inside the material. The origin is the exchange interaction, a quantum mechanical effect whose description and complete treatment can be found in $[1,19]$.

It all boils down to a spin dependent effective Hamiltonian, the Heisenberg Hamiltonian

$$
\hat{\mathcal{H}}=-2 \sum_{<i, j>} J_{i j} \mathbf{S}_{i} \cdot \mathbf{S}_{j}=-2 J S^{2} \sum_{<i, j>} \cos \phi_{i, j},
$$

where the sum is over first neighbours only, $J_{i j}$ is the exchange integral between spins $i$ and $j$ and $\phi_{i, j}$ is the angle between the two spins. Such interaction is of short range. When $J_{i j}>0$, the energy of the system is minimized when spins lie parallel to one another. The formulation of this requirement in the continuum considers that the angle between neighbouring spins is small so that the scalar product in (1.11) 
can be approximated as [1]

$$
\cos \phi_{i, j} \approx 1-\frac{\phi_{i, j}^{2}}{2}
$$

and the energy of the system becomes

$$
E=\text { constant }+J S^{2} \sum_{<i, j>} \phi_{i, j}^{2}
$$

The passage to the continuous approximation results in the energy density term

$$
\mathcal{E}_{\mathrm{ex}}=A\left[\left(\nabla m_{x}\right)^{2}+\left(\nabla m_{y}\right)^{2}+\left(\nabla m_{z}\right)^{2}\right]
$$

where the constant $A=2 J S^{2} z / a \mathrm{Jm}^{-1}$ is the exchange stiffness constant of the material, assuming a uniform exchange integral $J, a$ is the first neighbour distance and $z$ is a lattice constant that depends on the crystalline structure.

If we apply (1.10) to (1.12) we obtain the expression for the exchange contribution to the effective field.

$$
\mathbf{H}_{\mathrm{ex}}=-\frac{1}{\mu_{0} M_{s}} \frac{\delta \mathcal{E}_{\mathrm{ex}}}{\delta \mathbf{m}}=\frac{2 A}{\mu_{0} M_{s}} \nabla^{2} \mathbf{m}
$$

\subsubsection{Anisotropy}

Exchange interaction is isotropic, this means that in the ideal situation of a magnetic specimen whose spins are all aligned, the energy level will be the same regardless of the orientation of the magnetization $\mathrm{M}$. In the common case in which the specimen has a crystalline structure, the atoms, and hence the magnetic moments, are positioned at the vertices of the lattice. Due to spin-orbital interactions, orientations of magnetization along certain crystallographic axes are favoured with respect to others, breaking the symmetry of the isotropic exchange interaction. This effect is described by the so-called magnetocrystalline anisotropy.

Hexagonal and tetragonal crystals show a uniaxial anisotropy. Up to fourth order, the energy density dependence on the magnetization orientation can be written as

$$
\mathcal{E}_{\text {an }}=K_{u 1} \sin ^{2} \theta+K_{u 2} \sin ^{4} \theta .
$$

Here, $\theta$ is the angle between the magnetization direction and the anisotropy easy-axis $\hat{\mathbf{u}}$. The coefficients $K_{u 1}$ and $K_{u 2}$ are the anisotropy energy density constants $\left(\mathrm{J} \mathrm{m}^{-3}\right)$. Neglecting the fourth order terms this energy can be rewritten as

$$
\mathcal{E}_{\text {an }}=K_{u}\left(1-(\mathbf{m} \cdot \hat{\mathbf{u}})^{2}\right)
$$

since $\mathbf{m} \cdot \hat{\mathbf{u}}=\cos \theta$. A negative value of $K_{u}$ describes an easy-plane anisotropy, where all orientations of magnetization lying in the plane perpendicular to $\hat{\mathbf{u}}$ are equally favoured. On the other hand, a positive value of $K_{u}$ gives rise to a easy-axis anisotropy, where magnetization configurations parallel to $\hat{\mathbf{u}}$ have lower energy. In 
thin films, the coupling of few atomic layers of ferromagnetic metals with an oxide $[20,21]$ gives rise to a uniaxial anisotropy perpendicular to the interface between the two materials, whose strength scales with the inverse of the thickness of the ferromagnet. It is the case, for example, of $\mathrm{CoFeB} / \mathrm{MgO}$ interfaces. Here a surface anisotropy constant $K_{S}$ exists that adds to the bulk constant as

$$
K_{u}=K_{\mathrm{bulk}}+\frac{K_{S}}{t_{0}}
$$

where $t_{0}$ is the ferromagnet's thickness. In this thesis we will be dealing with the effects of surface anisotropy in $\mathrm{CoFeB} / \mathrm{MgO}$, including its effect directly in the value of $K_{u}$.

The effective field associated with anisotropy, derived using eq. (1.10) is

$$
\mathbf{H}_{\mathrm{an}}=\frac{2 K_{u}}{\mu_{0} M_{s}}(\mathbf{m} \cdot \hat{\mathbf{u}}) \hat{\mathbf{u}}
$$

\subsubsection{Zeeman energy}

In the simple case of the application of an external magnetic field $\mathbf{H}_{\mathrm{a}}$, generated from a coil or a magnet in the laboratory, we have already seen when discussing equilibrium conditions for magnetization that energy is minimized when $\mathbf{M}$ is parallel to $\mathbf{H}_{\mathrm{a}}$ : the so-called Zeeman energy density is given by

$$
\mathcal{E}_{\mathrm{Z}}=-\mu_{0} M_{s} \mathbf{m} \cdot \mathbf{H}_{\mathrm{a}} .
$$

The associated effective field is, obviously, $\mathbf{H}_{Z}=\mathbf{H}_{\mathrm{a}}$. The anticipation of this simple energy term due to an interaction external to the material is useful to describe the more complicated dipolar interaction, which is the energy term arising from the reciprocal interaction among the magnetic moments of a magnetic system.

\subsubsection{Magnetostatic energy}

Magnetostatic, demagnetizing, stray field, dipolar interaction. All these terms are used in the literature in different situations to describe the same interaction and associated energy and effective field. A point-like magnetic moment $\boldsymbol{\mu}$ generates a dipolar field

$$
\mathbf{H}(\mathbf{r})=\frac{1}{4 \pi}\left(\frac{3 \mathbf{r}(\boldsymbol{\mu} \cdot \mathbf{r})}{r^{5}}-\frac{\boldsymbol{\mu}}{r^{3}}\right) .
$$

If we consider a specimen of $n$ magnetic moments positioned in space, they will all interact with all the others giving an energy density that, using (1.17), reads

$$
\mathcal{E}_{\mathrm{d}}=-\frac{\mu_{0} M_{s}}{2} \sum_{i}^{n} \boldsymbol{\mu}_{i} \cdot\left(\sum_{j}^{n} \mathbf{H}_{j}\left(\mathbf{r}_{i}\right)\right) \text {. }
$$


where $\mathbf{H}_{j}\left(\mathbf{r}_{i}\right)$ is the dipolar field generated by the $j$-th magnetic moment evaluated at the $i$-th moment position. This rather complicated computation has workarounds, both from a theoretical point of view and from the computational one, as will be shown in the next chapter. Here it is sufficient to say that in the continuum approximation, from Maxwell's equations a scalar potential $\Phi$ can be extracted from the magnetization as

$$
\begin{aligned}
\Phi(\mathbf{r}) & =\frac{M_{s}}{4 \pi}\left[\int_{\Omega} \frac{\rho\left(\mathbf{r}^{\prime}\right)}{\left|\mathbf{r}-\mathbf{r}^{\prime}\right|} \mathrm{d} \mathbf{r}^{\prime}+\int_{\partial \Omega} \frac{\sigma\left(\mathbf{r}^{\prime}\right)}{\left|\mathbf{r}-\mathbf{r}^{\prime}\right|} \mathrm{d} \mathbf{r}^{\prime}\right] \\
\rho & =-\nabla \cdot \mathbf{m} \\
\sigma & =\mathbf{m} \cdot \hat{\mathbf{n}} .
\end{aligned}
$$

Here, $\rho$ is the so-called volume magnetic charge density and $\sigma$ is the surface charge density. Then $\mathbf{H}_{\mathrm{d}}=-\nabla \Phi$ and $\mathcal{E}_{\mathrm{d}}=-\frac{\mu_{0} M_{s}}{2} \mathbf{m} \cdot \mathbf{H}_{\mathrm{d}}$.

So far, inside a body uniformly magnetized along an anisotropy axis, $\mathbf{M}(\mathbf{r})=$ $\mathbf{M}_{0}$, the only non-zero effective field contribution is the dipolar field. In general, $\mathbf{H}_{\mathrm{d}}(\mathbf{r})$ is not uniform. However, in the particular case of an ellipsoid ${ }^{1}$, the internal field is uniform and depends on the direction of magnetization as

$$
\mathbf{H}_{\mathrm{d}}=-\overline{\mathbf{N}} \cdot \mathbf{M}_{0}
$$

where $\overline{\mathbf{N}}$ is a symmetric tensor called the demagnetizing tensor. The reason for this name is that if coordiantes are chosen along the principal exes of the ellipsoid, $\overline{\mathbf{N}}$ is diagonal, so that when the magnetization lies along one of the three axes of the ellipsoid, the tensor multiplication equals a scalar multiplication and $\mathbf{H}_{\mathrm{d}}$ results to be antiparallel to $\mathbf{M}$, thus opposing magnetization inside the sample. The three diagonal elements $N_{x}, N_{y}, N_{z}$, called demagnetizing factors, obey the constraint $N_{x}+$ $N_{y}+N_{z}=1$. In a uniformly magnetized ellipsoid the magnetostatic energy density is uniform over the sample and equal to:

$$
\mathcal{E}_{\mathrm{d}}=\frac{\mu_{0} M_{s}^{2}}{2} \mathbf{m} \cdot(\overline{\mathbf{N}} \cdot \mathbf{m})=\frac{\mu_{0} M_{s}^{2}}{2}\left(N_{x} m_{x}^{2}+N_{y} m_{y}^{2}+N_{z} m_{z}^{2}\right)
$$

If the ellipsoid has symmetry of revolution around the x axis, $N_{z}=N_{y}$ so that

$$
\begin{aligned}
\mathcal{E}_{\mathrm{d}} & =\frac{\mu_{0} M_{s}^{2}}{2}\left[N_{x} m_{x}^{2}+N_{y}\left(1-m_{x}^{2}\right)\right] \\
& =\frac{\mu_{0} M_{s}^{2}}{2}\left(N_{x}-N_{y}\right) m_{x}^{2}+\mathrm{const} \\
& =K_{0}(\mathbf{m} \cdot \hat{\mathbf{x}})^{2}+\mathrm{const}
\end{aligned}
$$

where

$$
K_{0}=\frac{\mu_{0} M_{s}^{2}}{2}\left(N_{x}-N_{y}\right)
$$

\footnotetext{
${ }^{1}$ It can be shown that this applies to any second-degree surface, however, the ellipsoid is the only one that is finite in space and hence has a practical relevance.
} 
This magnetostatic energy term has the form of a uniaxial anisotropy along the major axis of the sample (1.15). For this reason it is called shape anisotropy. In a prolate spheroid, where $x$ axis is longer than $y$ and $z$ axes, $N_{x}<N_{y}$ so that $K_{0}<0$ and energy is minimized when $\mathbf{m}$ lies along $x$ axis, which corresponds to the anisotropy easy axis. In an oblate spheroid, on the other hand, where $x$ axis is shorter than the other two, $K_{0}>0$ and shape anisotropy corresponds to an easy plane anisotropy.

Exchange, anisotropy, Zeeeman and demagnetizing are the energy terms usually considered in standard micromagnetics. Recently the fabrication of nanostructures with broken inversion symmetry led to the appearance of an additional interaction at the interfaces between magnetic materials and elements heavy metals, which turns out to have an important effect on magnetization dynamics. Therefore we present it in the following section.

\subsubsection{Dzyaloshinskii-Moriya interaction}

Exchange interaction has been presented with a fully symmetric Hamiltonian. However, its generalized form can be written as

$$
\mathcal{H}_{i j}=\mathbf{S}_{i} \cdot\left(\bar{M}_{i j} \mathbf{S}_{j}\right)
$$

where $\bar{M}_{i j}$ is a matrix representing the bilinear form of the energy of two spins. This can be decomposed in a symmetric and antisymmetric part. The symmetric part represents the usual exchange interaction that we presented as fully isotropic with a scalar product (1.11). The antisymmetric part can be rewritten as cross product by a vector $\mathbf{D}_{i j}$ :

$$
\mathbf{S}_{i} \cdot\left(\bar{M}_{i j} \mathbf{S}_{j}\right)=J_{i j} \mathbf{S}_{i} \cdot \mathbf{S}_{j}+\mathbf{S}_{i} \cdot\left(\mathbf{D}_{i j} \times \mathbf{S}_{j}\right) .
$$

The antisymmetric term is usually rewritten as

$$
\mathcal{H}_{i j}^{D M}=-\mathbf{D}_{i j} \cdot\left(\mathbf{S}_{i} \times \mathbf{S}_{j}\right) .
$$

This energy term is due to the presence of spin-orbit interaction, which connects the lattice with the spin symmetry. The broken parity of the lattice gives rise to an additional interaction that breaks the inversion invariance of the Heisenberg Hamiltonian, this is the Dzyaloshinskii-Moriya interaction (DMI). In the systems studied in this thesis, DMI is an interfacial effect, arising from the interaction of a ferromagnetic layer with an adjacent lattice of heavy metal atoms with large spin-orbit coupling (see fig. 1.4). The vector $\mathbf{D}$ is then determined by the interaction at the interface as $\propto \mathbf{r}_{i j} \times \hat{\mathbf{n}}$, where $\mathbf{r}_{i j}$ is the vector connecting spin $\mathbf{S}_{i}$ and $\mathbf{S}_{j}$ and $\hat{\mathbf{n}}$ is the interface plane vector.

As presented in fig. 1.4, we consider a broken inversion symmetry along the $\hat{z}$ direction, so that the DMI vector $\mathbf{D}$ is directed, for any in-plane direction $\hat{u}$, as $\mathbf{D}=$ $D \hat{z} \times \hat{u}$ [22]. In the continuous micromagnetic approximation, the energy density 


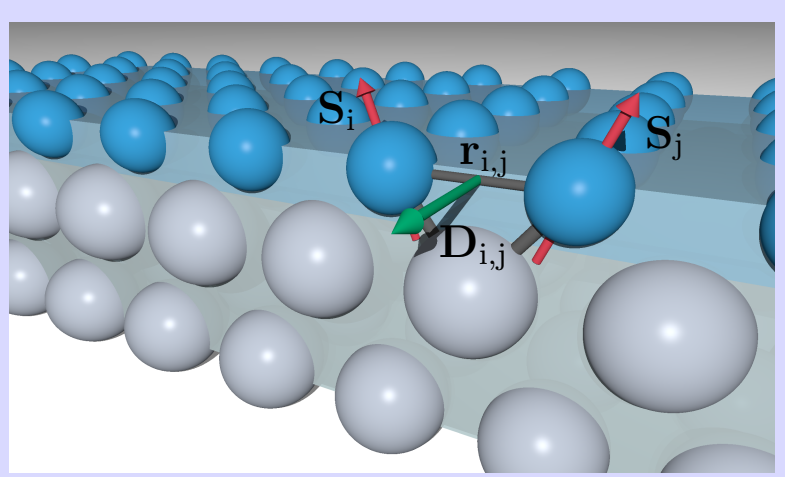

FIGURE 1.4: Sketch of DMI at the interface between a ferromagnetic metal (blue) and a heavy metal (grey). The DMI vector $\mathbf{D}_{i, j}$ related to the triangle composed of two magnetic sites $\mathbf{S}_{\mathrm{i}}$ and $\mathbf{S}_{\mathrm{j}}$ and an atom with a large spin orbit coupling (SOC) is perpendicular to the plane of the triangle.

term due to Dzyaloshinskii-Moriya interaction reads then

$$
\mathcal{E}_{\mathrm{DM}}=D\left[(\mathbf{m} \cdot \nabla) m_{z}-(\nabla \cdot \mathbf{m}) m_{z}\right] .
$$

Boundary conditions are also modified as [23]

$$
\frac{\partial \mathbf{m}}{\partial \mathbf{n}}=\frac{D}{2 A}(\hat{z} \times \mathbf{n}) \times \mathbf{m},
$$

where $\mathbf{n}$ is the vector normal to the surface. Finally, the effective DMI field is

$$
\mathbf{H}_{\mathrm{DM}}=\frac{2 D}{\mu_{0} M_{s}}\left[(\nabla \cdot \mathbf{m}) \hat{z}-\nabla m_{z}\right] .
$$

In conclusion, the energy density of a ferromagnetic specimen is

$$
\begin{aligned}
\mathcal{E}= & A \sum_{i=x, y, z}\left(\nabla m_{i}\right)^{2}+K_{u}\left(1-(\mathbf{m} \cdot \hat{\mathbf{u}})^{2}\right)-\frac{\mu_{0} M_{s}}{2} \mathbf{m} \cdot \mathbf{H}_{\mathrm{d}} \\
& -\mu_{0} M_{s} \mathbf{m} \cdot \mathbf{H}_{\mathrm{a}}+D\left[(\mathbf{m} \cdot \nabla) m_{z}-(\nabla \cdot \mathbf{m}) m_{z}\right] .
\end{aligned}
$$

According to (1.10), a general expression for the effective field can be obtained from (1.27), yielding

$$
\begin{aligned}
\mathbf{H}_{\mathrm{eff}}= & \frac{2 A}{\mu_{0} M_{s}} \nabla^{2} \mathbf{m}+\frac{2 K_{u}}{\mu_{0} M s}(\mathbf{m} \cdot \hat{\mathbf{u}}) \hat{\mathbf{u}}+\mathbf{H}_{\mathrm{d}} \\
& +\mathbf{H}_{\mathrm{a}}+\frac{2 D}{\mu_{0} M_{s}}\left[(\nabla \cdot \mathbf{m}) \hat{z}-\nabla m_{z}\right] .
\end{aligned}
$$




\subsection{From nanoparticles to mesoscale magnetism}

Solution of equation (1.8) and (1.9), that leads to equilibrium magnetization configurations is not a trivial task. Moreover, depending on the initial configuration, metastable states can be attained, which is the foundation of hysteretic behaviour in magnetism. In this section a qualitative description of the different effects of the various energy terms of a ferromagnetic system will provide the justification for the existence of magnetic domains and finally of domain walls, the main actors of magnetization dynamics at the mesoscale. To conclude this section, the concepts of domain wall width and energy are briefly explained.

Exchange interaction favours alignment of a spin parallel to its neighbours, regardless of their direction, whereas uniaxial anisotropy favours alignment with the easy axis of anisotropy. The application of an external field tends to align the magnetization with the external field. These are all short range energy terms, that depend on the orientation of the single spin or its direct neighbours at the most. The fact that domains exist in extended ferromagnets lies predominantly in the minimization of magnetostatic energy, which is a long range interaction. This means that, although at the local level its effect is negligible compared to local energy terms, it plays an important role when considering a large assembly of magnetic moments. The complicated nature of this interaction makes it difficult to schematically represent its minimization in a general way. However, by considering equations (1.19), (1.20) and (1.21), we can see that the two conditions to minimize magnetostatic energy are absence of volume magnetic charges $(\nabla \cdot \mathbf{m}=0)$ and absence of surface charges $(\mathbf{m} \cdot \mathbf{n}=0)$. This means that magnetostatic energy is minimized when magnetization configuration realizes a flux closure inside the volume of the magnetic specimen and is tangential to the surface of the sample. In order to do so, magnetization has to change direction inside the sample at a cost for anisotropy and exchange. Exchange interaction, however, depends on the angle between neighbouring spins so that, if the sample is large enough, the magnetization can change direction in space keeping a low gradient, which means a low exchange energy cost (1.12).

\subsubsection{Monodomains, vortices, multidomains}

In order to understand better the competition of different magnetic energies that gives rise to formation of domains in ferromagnets we will consider the following example. Let's consider tiles of Permalloy, a common NiFe alloy that has a negligible magnetocrystalline anisotropy, with aspect ratios $20 \delta: 10 \delta: \delta$ along the coordinate axes $x, y$ and $z$ respectively, and observe which is the equilibrium magnetization when minimizing energy starting from a random magnetic configuration. Fig. 1.5 shows the numerical results of this simple study. When $\delta=3 \mathrm{~nm}$, the sample is very small. Here exchange interaction dominates and an almost uniform state is formed. By looking at the energy density distribution, we see that exchange energy 
is minimized, while magnetic charges $\sigma \neq 0$ are formed at the edges of the magnet. This has a cost in dipolar energy for the system that, nevertheless, is small due to the small size of the sample.

In a bigger tile where $\delta=15 \mathrm{~nm}$, the situation is already much different, here magnetization prefers to bend smoothly, to be tangent to the sample edges and avoid surface charges. This curled configuration, called a vortex state, causes the tilt out of the plane of the magnetization at the centre, where, due to symmetry, there is no preferable in-plane direction. By looking at the magnetic energies distribution, $\mathcal{E}_{\text {ex }}$ is mostly concentrated at the vortex core, where magnetization is forced to rapidly curl and tilt out of plane. The almost constant angle between neighbouring spins in the rest of the sample has a small and almost uniform energy cost, only slightly increased along the diagonals. Dipolar energy density is also mostly concentrated at the vortex core, due to the surface charge generated by the out of plane spin at the center. Along the diagonals, the presence of volume charges where the magnetization direction changes with a larger angle is visible.

When the sample is even larger, $\delta=30 \mathrm{~nm}$, the situation is different again, here the flux closure is obtained via two adjacent vortices. However, the magnetization rotates now between one region to the next by a sharp $90^{\circ}$ rotation, leaving the magnetization in each region uniform, thus achieving flux closure while minimizing exchange energy too. By looking at the energy density distributions, energy results now concentrated in the two vortex cores and along the regions' boundaries, where the gradient of magnetization is larger. The regions of uniform magnetization in a magnetic sample are called magnetic domains, the interfaces between domains, where $\mathbf{M}$ rotates continuously from one direction to the other are called domain walls. Domain walls play a fundamental role in most magnetization dynamics phenomena.

The domains in fig. 1.5 are perpendicular to their neighbours, so that inside the domain walls magnetization rotates by $90^{\circ}$. The presence of a uniaxial anisotropy, of magnetocrystalline, interfacial or geometrical origin, favours the formation of antiparallel domains, so that domain walls carry a $180^{\circ}$ degree rotation of magnetization. This second type of domain wall will be the one studied in this thesis, so that it is useful to explore its properties in more detail.

\subsubsection{Landau and Lifshitz theory of domain walls}

We consider an infinite crystal with uniaxial anisotropy where magnetization is divided in two antiparallel domains. The magnetization aligns to the $\pm z$ direction imposed by anisotropy, and varies along the $x$ direction as shown in fig. 1.6. In the wall that separates the two domains, magnetization rotates from up to down direction, tilting away from the $z$ axis. This can, in principle, happen through any of the planes containing the $z$ axis. However, since our sample is infinite and we are assuming that the only direction along which magnetization varies is $x$. This means that zero volume charges $\nabla \cdot \mathbf{m}$ are achieved if $m_{x}=0$ always, i.e. magnetization rotates inside the $y z$ plane, as in fig. 1.6. This configuration has zero volume charges 


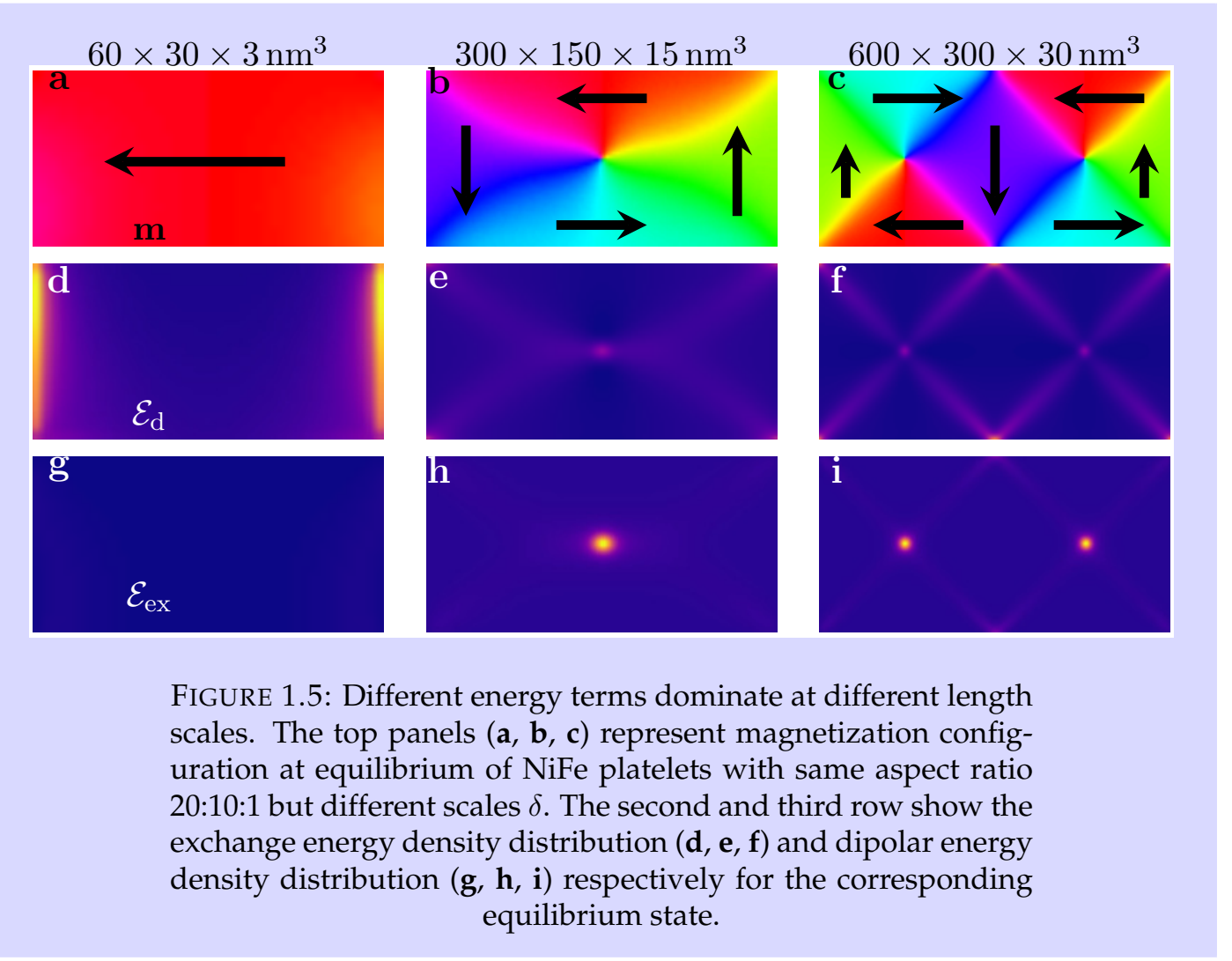

and is called a Bloch domain wall. It would lead to surface charges at the boundaries, but our assumption of an infinite crystal makes this energy term disappear too.

In such configuration there is no magnetostatic energy contribution at the domain wall and the only terms are due to anisotropy and exchange. The total energy density in this system is then

$$
\mathcal{E}=K_{u} m_{y}^{2}+A\left[\left(\frac{d m_{y}}{d x}\right)^{2}+\left(\frac{d m_{z}}{d x}\right)^{2}\right]
$$

This can be rewritten substituting $m_{z}=\cos \theta$ and $m_{y}=\sin \theta$. The total energy per unit area is obtained integrating along $x$ :

$$
E_{w}=\int_{-\infty}^{\infty}\left[K_{u} \sin ^{2} \theta+A\left(\frac{d \theta}{d x}\right)^{2}\right] d x
$$

It can be shown [19] that the configuration that minimizes this energy while respecting the fact that this is a domain wall $(\sin \theta=0$ for $x \rightarrow \pm \infty)$ is

$$
m_{z}(x)=\cos \theta(x)=-\tanh \left(\frac{x}{\Delta}\right),
$$

where the domain wall center is assumed to lie at $x=0 . \Delta$ is the domain wall width parameter, since it is the parameter that gives information about the extension of the region across which most of the rotation of magnetization is performed. The actual 
width of the domain wall can be estimated by first defining the domain wall as the only region where magnetization is not parallel to $z$ axis and thus integrating the transverse component of the magnetization $m_{\perp}=\sin \theta(x)$ along $x$ :

$$
\int_{-\infty}^{\infty} \sin \theta d x=\int_{0}^{\pi} \Delta d \theta=\pi \Delta
$$

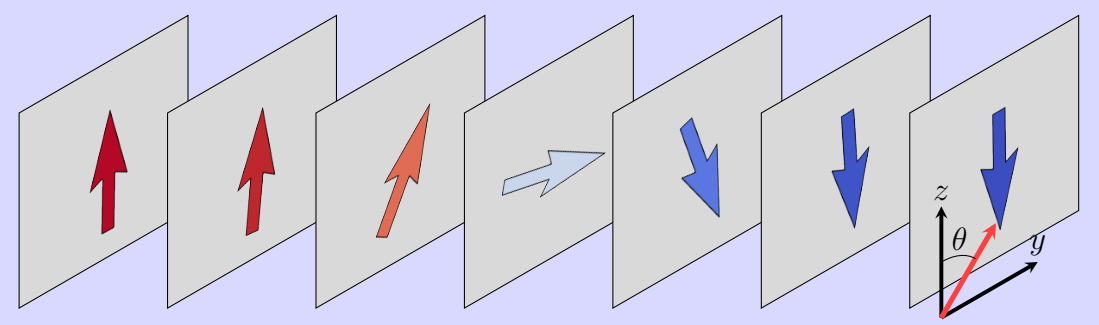

FIGURE 1.6: Representation of a Bloch domain wall in an infinite medium. Magnetization rotates between two antiparallel domains rotating inside the plane dividing the two domains or in other words, inside the plane perpendicular to the direction $x$, along which magnetization changes.

The domain wall width parameter $\Delta$ obtained here gives important information about the length scale over which magnetization can change direction in an equilibrium configuration. The value of $\Delta$ depends on the characteristics of the given system, since it represents the competition between exchange interaction trying to keep small angles between neighbouring spins and forces trying to curl magnetization as quickly as possible to align spins along a particular direction. In the case of a sample infinite along the three dimensions, such role is played exclusively by magnetocrystalline anisotropy and the value of $\Delta$ that minimizes (1.30) is

$$
\Delta=\sqrt{A / K_{u}}
$$

The value $\sqrt{A / K_{u}}$ is also called Bloch length $\ell_{B l o c h}$ of the system, since it is related with the Bloch domain wall configuration. Later on we will see that also magnetostatics can affect the domain wall width. Substituting the expression (1.31) into (1.30) and integrating along $x$ we obtain the domain wall surface energy density

$$
\sigma=4 \sqrt{A K_{u}}
$$

We consider now a different system, a wire infinite along $x$ but of limited cross section, magnetized along the wire axis, with a head to head domain wall separating antiparallel domains (see fig. 1.7), the magnetostatic energy can be interpreted as a shape anisotropy trying to align magnetization along the wire's axis. 


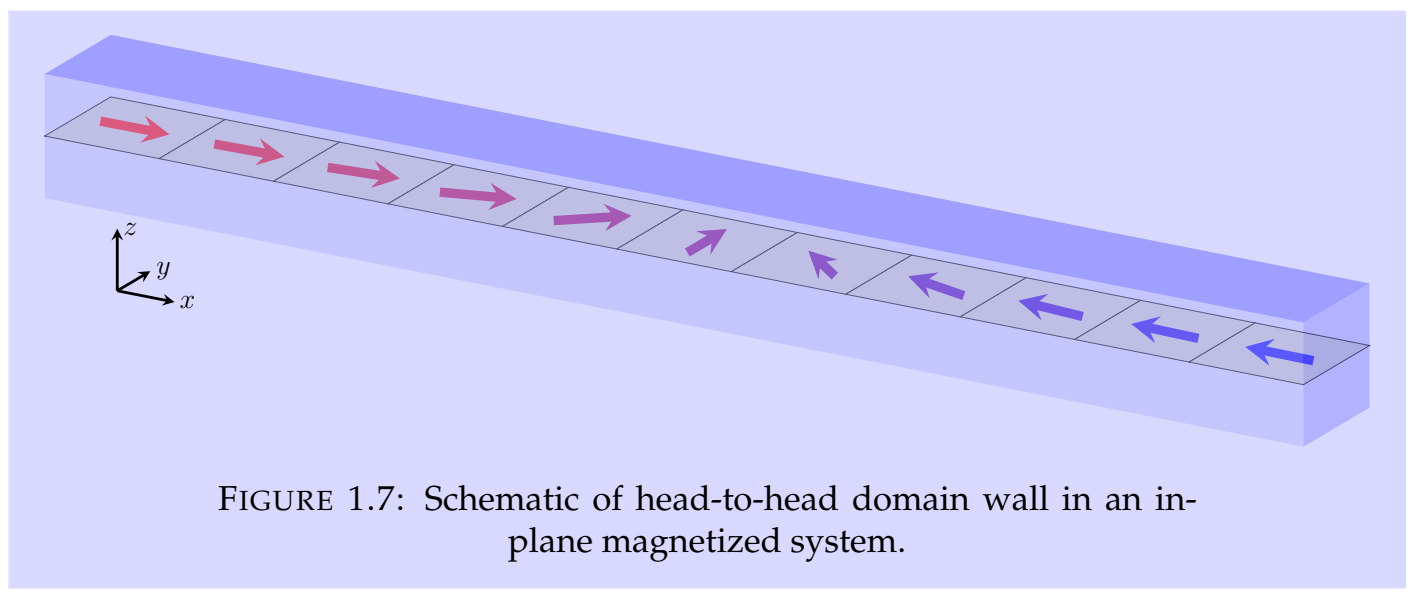

The energy of the system is then, in a first approximation

$$
\mathcal{E}=\frac{\mu_{0} M_{s}}{2} \mathbf{m} \cdot \mathbf{H}_{\mathrm{d}}+A\left[\left(\frac{d m_{x}}{d x}\right)^{2}+\left(\frac{d m_{\perp}}{d x}\right)^{2}\right] .
$$

Here the critical length describing the competition between exchange and shape anisotropy is the exchange length where the magnetocrystalline anisotropy constant is replaced by the shape anisotropy constant of demagnetizing origin (1.23) $K_{0}=$ $\frac{1}{2} \mu_{0} M_{s}^{2}$.

$$
\ell_{\mathrm{ex}}=\sqrt{2 A /\left(\mu_{0} M_{s}^{2}\right)}
$$

\subsection{Domain wall dynamics}

In this section domain wall dynamics is finally introduced. The simplest case of a domain wall moving due to the expansion of a domain parallel to an external field is discussed using energy dissipation arguments deriving directly from LLG equation. The modifications to domain wall dynamics in a disordered system is considered and the analytical theory useful to understand domain wall dynamics in presence of disorder is briefly presented.

\subsubsection{Energy minimization and field driven dynamics}

In the case where none of the energy densities depends explicitly on time, but only on $\mathbf{M}(t)$, the energy dissipation rate can be written as

$$
\begin{aligned}
\frac{\mathrm{d} E}{\mathrm{~d} t} & =\int_{V} \frac{\delta \mathcal{E}}{\delta \mathbf{M}} \cdot \frac{\mathrm{d} \mathbf{M}}{\mathrm{d} t} \mathrm{~d} V \\
& =-\mu_{0} \int_{V}\left(\mathbf{H}_{\text {eff }} \cdot \frac{\mathrm{d} \mathbf{M}}{\mathrm{d} t}\right) \mathrm{d} V
\end{aligned}
$$


On the other hand, multiplying LLG equation (1.6), by $\mathbf{H}_{\text {eff }}$ we obtain

$$
\mathbf{H}_{\mathrm{eff}} \cdot \frac{\mathrm{d} \mathbf{M}}{\mathrm{d} t}=\frac{\alpha}{M_{s}} \mathbf{H}_{\mathrm{eff}} \cdot\left(\mathbf{M} \times \frac{\mathrm{d} \mathbf{M}}{\mathrm{d} t}\right),
$$

while multiplying it by $\frac{\mathrm{dM}}{\mathrm{d} t}$ yields

$$
\left(\frac{\mathrm{d} \mathbf{M}}{\mathrm{d} t}\right)^{2}=-\gamma_{0} \frac{\mathrm{d} \mathbf{M}}{\mathrm{d} t} \cdot\left(\mathbf{M} \times \mathbf{H}_{\mathrm{eff}}\right) .
$$

From (1.36) and (1.37), we get the relation

$$
\mathbf{H}_{\mathrm{eff}} \cdot \frac{\mathrm{d} \mathbf{M}}{\mathrm{d} t}=\frac{\alpha}{\gamma_{0} M_{s}}\left(\frac{\mathrm{d} \mathbf{M}}{\mathrm{d} t}\right)^{2}
$$

This relation is valid at every point of our system and at every instant. By substituting equation (1.38) in (1.35) we obtain a relation for the total energy dissipation rate of our magnetic system:

$$
\frac{\mathrm{d} E}{\mathrm{~d} t}=-\frac{\alpha \mu_{0}}{\gamma_{0} M_{s}} \int_{V}\left(\frac{\mathrm{d} \mathbf{M}}{\mathrm{d} t}\right)^{2} \mathrm{~d} V
$$

This is the amount of magnetic energy that is transferred to the lattice due to dissipative effects during magnetization dynamics, it has no local counterpart because the energy lost locally by a single spin is not entirely transferred to the lattice but can go increasing the magnetic energy of other spins the system.

Let's consider an infinite sample divided in two antiparallel domains, separated by a $180^{\circ}$ domain wall as the one represented in fig. 1.6. By applying an external magnetic field $\mathbf{H}_{a}$, constant in time and uniform along the $+z$ axis, we modify the energy density of the system. A term describing the Zeeman energy needs to be added to eq. (1.30):

$$
E_{w}=\int_{-\infty}^{\infty}\left[K_{u} \sin ^{2} \theta+A\left(\frac{d \theta}{d x}\right)^{2}-\mu_{0} M_{s} H_{a} \cos \theta\right] d x
$$

Since $H_{a}$ is chosen positive, the energy of the system is minimum when $\cos \theta=1$ for all the spins in the system. This can be achieved by flipping of the spins in the right domain or by the expansion of the left domain and the shrinking of the right one. It is proven both experimentally and theoretically, that for systems larger than a critical size and for applied fields below a certain (high) threshold, the latter process is the one taking place [17]. In fact, here we are interested in this second situation, which results in domain wall motion. 


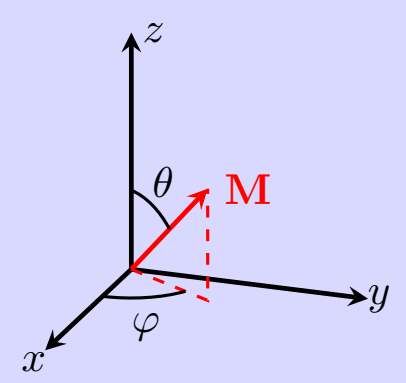

FIGURE 1.8: Polar (spherical) coordinate system for magnetization. The radius is fixed as $M_{s}$ for $\mathbf{M}$ and to 1 for $\mathbf{m}$. The polar angle is $\theta$ and the azimuthal angle is $\varphi$.

LLG equation (1.6) can be expressed in polar coordinates $\theta(\mathbf{r}, t), \varphi(\mathbf{r}, t)$ for the magnetization, as shown in fig. 1.8

$$
\begin{aligned}
\dot{\theta} & =-\frac{\gamma_{0}}{\mu_{0} M_{s} \sin \theta} \frac{\delta \mathcal{E}}{\delta \varphi}-\alpha \dot{\varphi} \sin \theta, \\
\dot{\varphi} \sin \theta & =\frac{\gamma_{0}}{\mu_{0} M_{s}} \frac{\delta \mathcal{E}}{\delta \theta}+\alpha \dot{\theta}
\end{aligned}
$$

where the dot indicates time derivative and

$$
\mathbf{H}_{\mathrm{eff}}=-\frac{1}{\mu_{0} M_{s}} \frac{\delta \mathcal{E}}{\delta \theta} \hat{\theta}-\frac{1}{\mu_{0} M_{s} \sin \theta} \frac{\delta \mathcal{E}}{\delta \varphi} \hat{\varphi} .
$$

In this notation, time variation of magnetic energy density $\dot{\mathcal{E}}$ can be written as:

$$
\dot{\mathcal{E}}=\frac{\delta \mathcal{E}}{\delta \theta} \dot{\theta}+\frac{\delta \mathcal{E}}{\delta \varphi} \dot{\varphi}=-\frac{\alpha \mu_{0} M_{s}}{\gamma_{0}}\left[\dot{\theta}^{2}+\dot{\varphi}^{2} \sin ^{2} \theta\right] .
$$

This is the polar coordinates equivalent of equation (1.38), describing energy conservation in our system: at every point, at every instant of time, variation of internal magnetic energy density equals the energy density dissipation at that point, at that instant. This is not a local relationship because inside $\frac{\delta \mathcal{E}}{\delta \theta}$ and $\frac{\delta \mathcal{E}}{\delta \varphi}$ there are non local dependencies on $\theta$ and $\varphi$, such as long range dipolar interaction and exchange interaction that depends on magnetization gradient. In order to extract information on domain wall dynamics from this relationship, we need to simplify the problem introducing an adequate approximation: the one dimensional model.

\subsubsection{One dimensional model}

It has been shown that in an infinite sample whose magnetization consists of two domains anti-parallel to each other, magnetization orientation is uniform inside the $y z$ plane defined by the domain wall and changes only along the direction $x$ perpendicular to the wall plane. We called this equilibrium configuration the Bloch domain wall. This means that $\varphi=\frac{\pi}{2}$ or $-\frac{\pi}{2}$ over the whole sample. 
The application of an external field along the $z$ direction does not exert any torque on the spins inside the domains since $\mathbf{m} \| \mathbf{H}_{\mathrm{a}}$. At the domain wall however, this is not true and a tilt of $\mathbf{m}$ inside the $x y$ plane will take place, described by a change in $\varphi$ for the spins inside the domain wall. We assume then that magnetization is described by the Bloch profile (1.31) in the out of plane direction, with an internal domain wall angle $\phi$ describing the $x y$-plane orientation of the spins inside the domain wall. We assume:

$$
\begin{aligned}
& \theta(\mathbf{r}, t)=2 \arctan \left[\exp \left(\frac{x-q(t)}{\Delta}\right)\right], \\
& \varphi(\mathbf{r}, t)=\phi(t),
\end{aligned}
$$

where $\Delta$ is the domain wall width parameter and $q$ is the parameter describing the domain wall position along the $x$ direction. We obtain the relationships

$$
\begin{array}{lll}
\frac{\partial \theta}{\partial x}=\frac{\sin \theta}{\Delta} & \delta \theta=-\frac{\sin \theta}{\Delta} \mathrm{d} q & \dot{\theta}=-\dot{q} \frac{\sin \theta}{\Delta} \\
\frac{\partial \varphi}{\partial x}=0 & \delta \varphi=\mathrm{d} \phi & \dot{\varphi}=\dot{\phi}
\end{array}
$$

Additionally, we consider a system with boundaries: an infinite wire along the $x$ direction. This introduces in the system dipolar energy in the form of surface magnetic charges at the boundaries of the domain wall that affect both the statics and the dynamics. However, the assumption that magnetization is uniform along $y$ and $z$ will hold as long as the cross section is small.

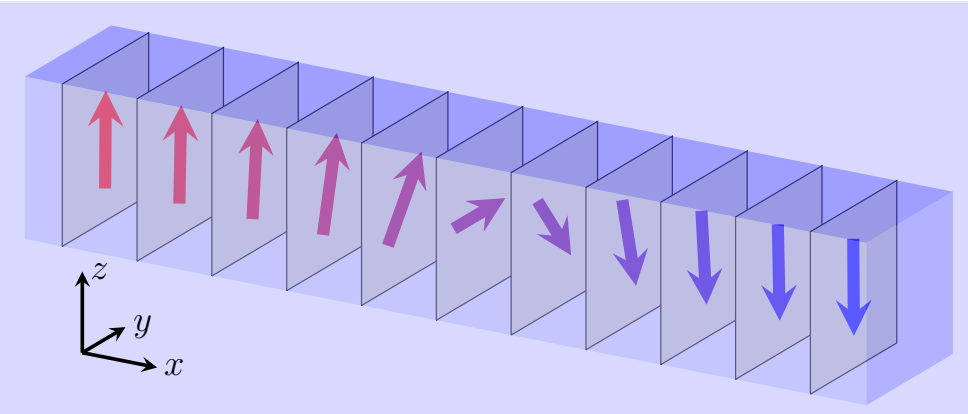

FIGURE 1.9: One dimensional representation of a wire of infinite length magnetized in two anti-parallel domains separated by a Bloch wall. Magnetization varies along $x$ while it is uniform over the cross section. 
Energy densities in polar coordinates are:

$$
\begin{aligned}
\mathcal{E}_{\text {ex }} & =A\left[(\nabla \theta)^{2}+(\nabla \varphi)^{2} \sin ^{2} \theta\right] \\
\mathcal{E}_{a n} & =K_{u} \sin ^{2} \theta \\
\mathcal{E}_{d} & =\frac{\mu_{0} M_{s}^{2}}{2}\left(N_{z}+\sin ^{2} \theta\left(N_{x}-N_{z}\right)+\sin ^{2} \theta \sin ^{2} \varphi\left(N_{y}-N_{x}\right)\right) \\
\mathcal{E}_{z} & =-\mu_{0} M_{s} H_{a} \cos \theta
\end{aligned}
$$

Assuming the rigid magnetic configuration described by eq. (1.44) and (1.45), the variation $\delta \mathcal{E}$ of $\mathcal{E}$ is much easier to write, since $\mathcal{E}$ reduces to

$$
\mathcal{E}=A \frac{\sin ^{2} \theta}{\Delta^{2}}+\sin ^{2} \theta\left(K_{0}+K \sin ^{2} \phi\right)-\mu_{0} M_{s} H_{a} \cos \theta,
$$

where we write an effective anisotropy term as

$$
\mathcal{E}_{a n}+\mathcal{E}_{d}=\underbrace{\left(K_{u}+\frac{1}{2} \mu_{0} M_{s}^{2}\left(N_{x}-N_{z}\right)\right)}_{K_{0}} \sin ^{2} \theta+\underbrace{\left(\frac{1}{2} \mu_{0} M_{s}^{2}\left(N_{y}-N_{x}\right)\right)}_{K} \sin ^{2} \theta \sin ^{2} \phi
$$

and we neglect the constant term $\frac{\mu_{0} M_{s}^{2}}{2} N_{z}$ from energy calculations. The hard-axis anisotropy constant $K$ has a sign that depends on the cross section of the wire. If $K$ is negative, the DW plane is an easy plane, and the wall has a Bloch configuration. When $K$ is positive, on the other hand, the wire axis is an easy axis for the domain wall spins and the domain wall minimum energy state will be that of a Néel domain wall.

We already know that, in general, from LLG equations (1.41), (1.42):

$$
\begin{aligned}
\delta \mathcal{E} & =\frac{\delta \mathcal{E}}{\delta \theta} \delta \theta+\frac{\delta \mathcal{E}}{\delta \varphi} \delta \varphi \\
& =-\frac{\mu_{0} M_{s}}{\gamma_{0}}[(\alpha \dot{\theta}-\dot{\varphi} \sin \theta) \delta \theta+(\dot{\theta}+\alpha \dot{\varphi} \sin \theta) \sin \theta \delta \varphi] .
\end{aligned}
$$

We eliminate the dependence on $\theta$ upon integration over $x$ :

$$
\begin{aligned}
\mathrm{d} \sigma & =\int_{-\infty}^{\infty} \delta \mathcal{E} \mathrm{d} x \\
& =-\frac{\mu_{0} M_{s}}{\gamma_{0}}\left[\int_{-\infty}^{\infty}(\alpha \dot{\theta}-\dot{\varphi} \sin \theta) \delta \theta \mathrm{d} x+\int_{-\infty}^{\infty}(\dot{\theta}+\alpha \dot{\varphi} \sin \theta) \sin \theta \delta \varphi \mathrm{d} x\right](1.5)
\end{aligned}
$$

To do so easily, we plug in the expressions obtained with the rigid model (1.46) and (1.47) to have

$$
\begin{aligned}
& \mathrm{d} \sigma=-\frac{\mu_{0} M_{s}}{\gamma_{0}}\left[\left(\alpha \frac{\dot{q}}{\Delta}+\dot{\phi}\right) \mathrm{d} q \int_{-\infty}^{\infty} \frac{\sin ^{2} \theta}{\Delta} \mathrm{d} x+\left(-\frac{\dot{q}}{\Delta}+\alpha \dot{\phi}\right) \mathrm{d} \phi \int_{-\infty}^{\infty} \sin ^{2} \theta \mathrm{d} x\right] \\
& \mathrm{d} \sigma=-\frac{2 \mu_{0} M_{s}}{\gamma_{0}}\left[\left(\alpha \frac{\dot{q}}{\Delta}+\dot{\phi}\right) \mathrm{d} q+(-\dot{q}+\alpha \Delta \dot{\phi}) \mathrm{d} \phi\right]
\end{aligned}
$$


The linear wall energy $\sigma\left(\mathrm{J} \mathrm{m}^{-2}\right)$ is simply

$$
\sigma=\int_{-\infty}^{\infty} \mathcal{E} \mathrm{d} x=\frac{2 A}{\Delta}+2 \Delta\left(K_{0}+K \sin ^{2} \phi\right)-2 q M_{s} H_{a}
$$

so that

$$
\mathrm{d} \sigma=\frac{\mathrm{d} \sigma}{\mathrm{d} q} \mathrm{~d} q+\frac{\mathrm{d} \sigma}{\mathrm{d} \phi} \mathrm{d} \phi=-2 \mu_{0} M_{s} H_{a} \mathrm{~d} q+2 \Delta K \sin (2 \phi) \mathrm{d} \phi .
$$

Equating the differentials in the two expressions of $\mathrm{d} \sigma,(1.58)$ and (1.60), we obtain the two one dimensional model equations:

$$
\begin{aligned}
-\frac{2 \mu_{0} M_{s}}{\gamma_{0}}\left(\alpha \frac{\dot{q}}{\Delta}+\dot{\phi}\right) & =-2 \mu_{0} M_{s} H_{a} \\
-\frac{2 \mu_{0} M_{s}}{\gamma_{0}}(-\dot{q}+\alpha \Delta \dot{\phi}) & =2 \Delta K \sin (2 \phi)
\end{aligned}
$$

rewritten as

$$
\begin{aligned}
\alpha \frac{\dot{q}}{\Delta}+\dot{\phi} & =\gamma_{0} H_{a} \\
\frac{\dot{q}}{\Delta}-\alpha \dot{\phi} & =\frac{2 \gamma_{0} K}{\mu_{0} M_{s}} \frac{\sin (2 \phi)}{2}
\end{aligned}
$$

It is useful to decouple the two equations

$$
\begin{aligned}
& \dot{q}=\frac{\gamma_{0} \Delta}{1+\alpha^{2}}\left(\alpha H_{a}+H_{K} \frac{\sin (2 \phi)}{2}\right) \\
& \dot{\phi}=\frac{\gamma_{0}}{1+\alpha^{2}}\left(H_{a}-\alpha H_{K} \frac{\sin (2 \phi)}{2}\right) .
\end{aligned}
$$

Where we introduced the shape anisotropy field

$$
H_{K}=\frac{2 K}{\mu_{0} M_{s}} .
$$

Moreover, the domain wall width parameter that minimizes the energy of the system is found imposing $\frac{\mathrm{d} \sigma}{\mathrm{d} \Delta}=0$ :

$$
\Delta=\sqrt{\frac{A}{K_{0}+K \sin ^{2} \phi}} .
$$

$\Delta$ is a time varying parameter since it depends on $\phi(t)$. It is useful to define the static domain wall width $\Delta_{0}=\Delta(\phi=\pi / 2)=\sqrt{A /\left(K_{0}+K\right)}$.

Equation (1.65) is the expression of the instantaneous velocity of the domain wall, which is constituted by a constant term dependent on the external field and a time dependent contribution due to the shape anisotropy field and the in-plane orientation of the domain wall. These equations prescribe two different dynamic regimes of propagation for the domain wall. 
Energy dissipation rate in the simplified system is described by:

$$
\begin{aligned}
\dot{\sigma} & =\frac{\mathrm{d} \sigma}{\mathrm{d} q} \dot{q}+\frac{\mathrm{d} \sigma}{\mathrm{d} \phi} \dot{\phi} \\
& =-2 \mu_{0} M_{s} H_{a} \dot{q}+2 \Delta K \sin (2 \phi) \dot{\phi},
\end{aligned}
$$

whereas energy conservation equation (1.43) gives:

$$
-2 \mu_{0} M_{s} H_{a} \dot{q}+2 \Delta K \sin (2 \phi) \dot{\phi}=\frac{-2 \alpha \mu_{0} M_{s}}{\gamma_{0}}\left[\frac{\dot{q}^{2}}{\Delta}+\Delta \dot{\phi}^{2}\right]
$$

If we assume that the domain wall is not precessing, $\dot{\phi}=0$, then it has a constant velocity

$$
v=\frac{\gamma_{0} \Delta}{1+\alpha^{2}}\left(\alpha H_{a}+H_{K} \frac{\sin \left(2 \phi^{*}\right)}{2}\right) .
$$

The value $\phi^{*}$ is obtained equating eq. (1.66) to zero, yielding

$$
\phi^{*}=\frac{1}{2} \arcsin \left(\frac{2 H_{a}}{\alpha H_{K}}\right) \text {, }
$$

which means that a constant solution to (1.66) exists only if $\left|H_{a}\right| \leq \frac{\alpha H_{K}}{2}$. For such applied fields, the domain wall velocity is directly proportional to the applied field and inversely proportional to Gilbert's damping $\alpha$.

$$
v=\frac{\gamma_{0} \Delta}{\alpha} H_{a}
$$

The limit field $H_{W}=\frac{\alpha H_{K}}{2}$ is called Walker field.

We can obtain eq. (1.72) directly from the energy conservation equation (1.71) substituting $\dot{\phi}=0$

$$
\begin{aligned}
-2 \mu_{0} M_{s} H_{a} v & =\frac{-2 \alpha \mu_{0} M_{s}}{\gamma_{0}} \frac{v^{2}}{\Delta} \\
v & =\frac{\gamma_{0} \Delta}{\alpha} H_{a},
\end{aligned}
$$

meaning that in this regime, since no energy is dissipated through precession of the spins inside the domain wall, the Zeeman energy is minimized solely by the domain wall displacement. The efficiency of an external field at moving the domain wall is described by the domain wall mobility $\mu$, which in this regime is

$$
\mu=\frac{v}{H_{a}}=\frac{\gamma_{0} \Delta}{\alpha} .
$$

For applied fields above $H_{W}$ there is no constant solution to (1.65) and the domain wall spins precess in the plane, giving rise to a time-varying velocity. Equation 
(1.66) has analytical solution [24]:

$$
\phi(t)=\arctan \left[\left(\tan \left[\left(\frac{\gamma_{0} H_{W}}{1+\alpha^{2}} t+C_{0}\right) \sqrt{h^{2}-1}\right] \sqrt{h^{2}-1}+1\right) \frac{1}{h}\right],
$$

where $h=H_{a} / H_{W}$. The period of precession is

$$
T=\frac{2 \pi\left(1+\alpha^{2}\right)}{\gamma_{0} H_{W} \sqrt{h^{2}-1}} .
$$

For an applied field above $H_{W}$ the averaged domain wall velocity reads

$$
\begin{aligned}
v & =\langle\dot{q}\rangle_{T}=\frac{1}{T} \int_{T} \dot{q} \mathrm{~d} t= \\
& =\frac{\alpha \gamma_{0} \Delta}{1+\alpha^{2}} H_{a}+\frac{\gamma_{0} \Delta}{\alpha\left(1+\alpha^{2}\right)}\left[H_{a}-\sqrt{H_{a}^{2}-H_{W}^{2}}\right] .
\end{aligned}
$$

In the limit for $H_{a} \gg H_{W}$ the domain wall average velocity becomes linear with the applied field as

$$
v=\frac{\alpha \gamma_{0} \Delta}{1+\alpha^{2}} H_{a},
$$

with a mobility $\mu=\alpha \gamma_{0} \Delta\left(1+\alpha^{2}\right)^{-1}$. Since usually $\alpha<1$, this regime has a mobility lower than the rigid regime below Walker field by a factor $\alpha^{2}\left(1+\alpha^{2}\right)^{-1}$. The two regimes of field-driven domain wall motion described by the one dimensional model are called throughout this thesis the rigid or linear regime when $\dot{\phi}=0$ and the precessional regime above Walker field.

To conclude this treatment on domain wall dynamics, we would like to exploit the simplicity of the one dimensional model to give a deeper understanding on the competition of the different torques acting on the domain wall and how this generates two different regimes. If we take into consideration only the spin at the center of the domain wall, this lies in the $x y$ plane and four different torques act on it. These torques are the precessional and damping torque of both the external field and of the demagnetizing field. It is helpful to rewrite equation (1.65) as

$$
-\sin \theta \dot{\theta}=\frac{\dot{q}}{\Delta}=\frac{\gamma_{0}}{1+\alpha^{2}}\left(\alpha H_{a}+H_{K} \frac{\sin (2 \phi)}{2}\right)
$$

From equation (1.66), the applied field exerts a torque in plane $\tau_{a}=\frac{\gamma_{0}}{1+\alpha^{2}} H_{a}$ that rotates the spin counter-clockwise as shown in figure 1.10-a. At the same time, the damping torque $\tau_{a}^{\alpha}=\alpha \tau_{a}$ rotates the spin out of the plane, effectively moving forward the domain wall. As soon as the domain wall spin is not aligned in the Bloch orientation inside the $y z$ plane, volume magnetostatic charges form, this magnetostatic field is described in the one dimensional model as the demagnetizing field of a uniformly magnetized ellipsoid, in fact we described demagnetizing energy using demagnetizing factors. The magnitude of this field depends on the rotation of the 
domain wall and is $H_{K} \frac{\sin 2 \phi}{2}$. Its orientation is along the $x$ axis, so that its precessional torque $\tau_{d}=\frac{\gamma_{0}}{1+\alpha^{2}} H_{K} \frac{\sin 2 \phi}{2}$ is oriented perpendicular to the $x y$ plane, as shown in figure 1.10-b. The damping torque associated to this field $\tau_{d}^{\alpha}$, on the other hand, is perpendicular to the plane of $\mathbf{m}$ and $z$ axis, so it has the same direction of the applied field precessional torque but opposite sign. The strength of the in plane torque $\tau_{d}^{\alpha}$, opposing the precession induced by $\tau_{a}$, grows while the domain wall orientation changes from $\phi=\pi / 2$ to $\phi=3 \pi / 4$, eventually blocking the precession and stabilizing the in-plane domain wall configuration. This results in the rigid motion regime below Walker field. If the applied field is larger than the maximum strength of the magnetostatic field, achieved at $\phi=3 \pi / 4$, then precession cannot be stopped by the magnetostatic damping torque $\tau_{d}^{\alpha}$ and precessional regime is attained. The demagnetizing torques change sign alternately in the quadrants since their sign depend on $\sin 2 \theta$, as shown in fig. 1.10, so that there is an alternate contribution to the domain wall motion. For applied fields close to $H_{W}$ this can cause negative values of instantaneous velocity, but the averaged contribution to the domain wall motion over $2 \pi$ is, however, always positive. The precession rate $\dot{\phi}$ is not constant in time, and domain wall rotation is slower in the II and IV quadrants, where $\tau_{d}^{\alpha}$ opposes $\tau_{a}$ and slows down precession.
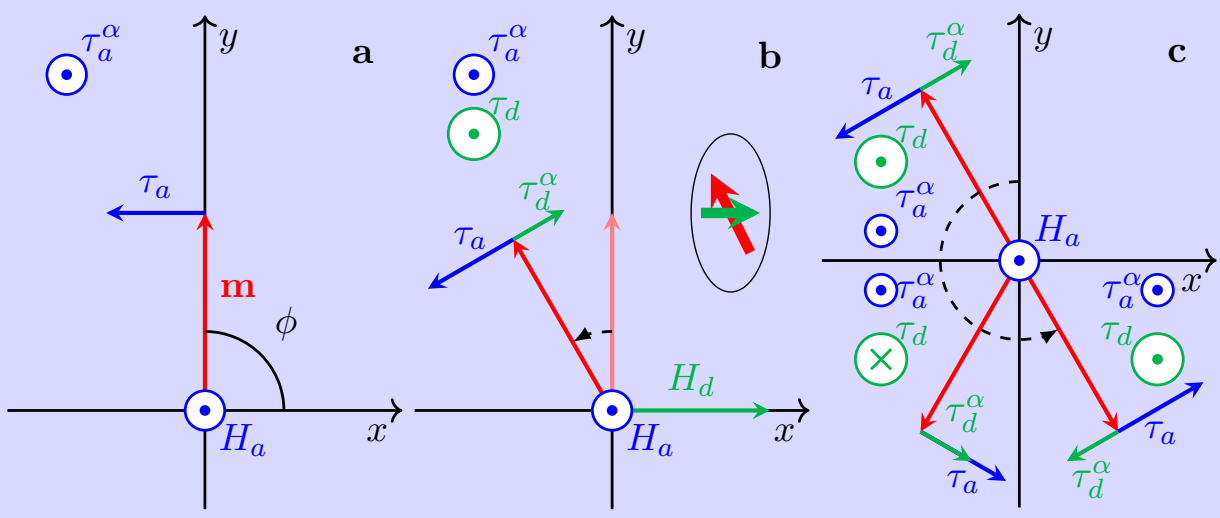

FIGURE 1.10: The torques acting on the domain wall according to the 1D model. The action of the external and dipolar fields expressed as the separate torques exerted on the magnetization at the center of the domain wall. a At rest, no magnetic charges are present, the only acting torques are those due to external applied field $\mathbf{H}_{\mathrm{a}}$. b The in-plane rotation of the domain wall produced by the external field creates magnetic charges and an inplane dipolar field acts on the magnetization producing an out of plane torque and a damping torque opposing in-plane precession. If applied field is below Walker field, an equilibrium is reached between $\tau_{a}$ and $\tau_{d}^{\alpha}$ so that domain wall moves driven by the sum of the two out of plane torques. $\mathrm{c}$ If the external field is above Walker field, the damping torque of the local stray field is not strong enough to stop precession and domain wall precesses in-plane. The motion is oscillatory and non-uniform since the driving torques $\tau_{a}^{\alpha}$ and $\tau_{d}$ are alternatively parallel in II and IV quadrant and anitparallel in the I and III quadrants. 
In figure 1.11 we plot the domain wall velocity as function of field from equation (1.72) and (1.76), the two different colors show the contributions to domain wall displacement due to the two different torques: the contribution from $\tau_{a}^{\alpha}$, shown in blue, is linear with the applied field, while the contribution from $\tau_{d}$, shown in green, plays the most important role in the rigid regime, while its contribution drops after Walker's field, when domain wall precession takes place, and vanish at very high fields when domain wall precession becomes almost uniform.

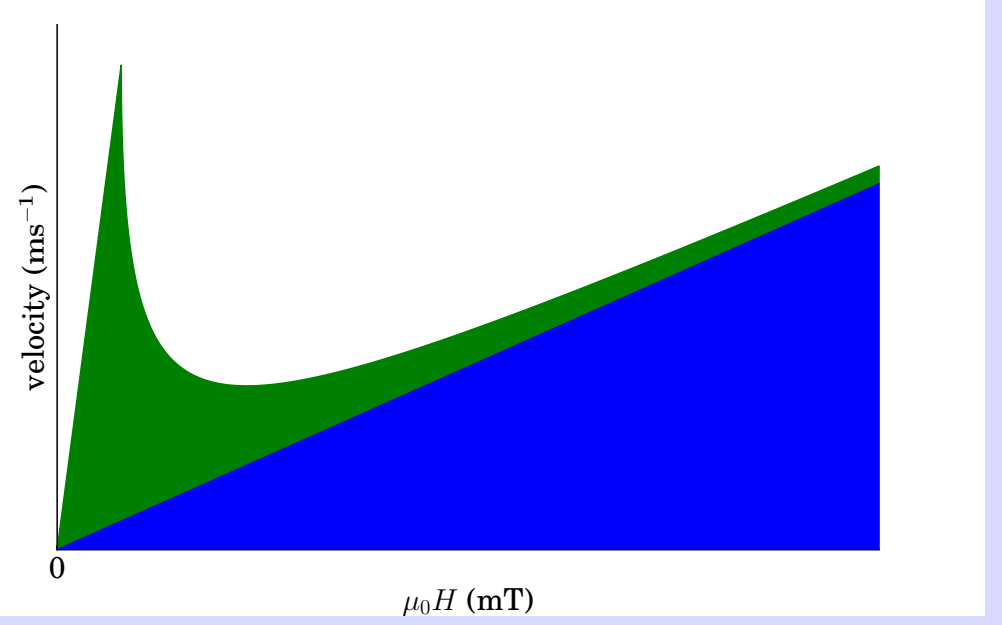

FIGURE 1.11: Domain wall velocity as function of the applied field as predicted by one dimensional model. The blue part is the contribution to DW velocity from the external field torque and the green part is the contribution from the demagnetizing field torque. As can be observed, the dipolar field torque contribution is dominant in the linear regime while decresing in the precessional regime. At high fields, where precession rate is almost constant, the positive and negative contributions of the demagnetizing torque cancel out.

Since we have seen that also the domain wall width parameter is time-dependent, the same argument used to derive equations (1.63) and (1.64) can be modified to include the variation of linear energy $\sigma$ with $\Delta$ and obtain a third equation for dynamics

$$
\dot{\Delta}=\frac{12 \gamma_{0}}{\alpha \mu_{0} M_{s} \pi^{2}}\left(\frac{A}{\Delta}-\Delta\left(K_{0}+K \sin ^{2} \phi\right)\right) .
$$

The inclusion of this time-varying parameter does not affect qualitatively the domain wall dynamics described so far. It adds to the dynamics the change in width of the domain wall that evolves towards an equilibrium width $\Delta^{*}=\Delta\left(\phi^{*}\right)$ in the rigid regime or periodically widens and narrows with a breathing mode in the precessional regime. 


\section{Chapter 2}

\section{Numerical simulations}

As it has been presented in chapter 1, micromagnetics is based on a continuum approximation of inter-atomic exchange interactions and on Maxwell's equations in the static limit for the evaluation of demagnetizing field. The integration of LandauLifshitz-Gilbert equation gives a precise description of magnetization distribution in finite bodies both in space and time. However, analytical solutions are hardly found $[19,17]$ and, in order to investigate magnetization dynamics in realistic and useful systems, numerical approximation are unavoidable. Numerical micromagnetic solvers are an important tool that connect theoretical predictions with experimental measurements. This chapter is composed of two parts, in the first one we present the structure of the micromagnetic solver developed to obtain the results this thesis. The second section contains no physics but introduces the interested reader to parallel computing with CUDA, the language in which the micromagnetic solver is written.

\subsection{Micromagnetic solver}

To integrate a differential equation such as (1.6) we need to discretize the volume of the sample so that the continuous vector field of magnetization $\mathbf{M}$ is sampled at a finite set of points, converting the problem into a finite set of coupled equations that are integrated in time simultaneously. Two different approaches exist for discretization: the finite-difference approach [25, 26, 27, 14, 28, 29], which divides the sample in a rectangular mesh, sampling $\mathbf{M}$ at equispaced nodes, and the finiteelement approach [30,31,32], where space is subdivided in non-uniform tetrahedral mesh. Both methods present advantages and disadvantages. The most computationally demanding part of a micromagnetic solver is the evaluation of the demagnetizing field, whose computation can be accelerated in a finite-difference solver. Finite-element irregular meshing, on the other hand, produces a more precise discretization of boundaries and better resolution of magnetization dynamics in samples with curved surfaces. The micromagnetic solver used in this work is based on the finite-difference approach. 


\subsubsection{Finite differences}

In a finite-difference approach to solve equation (1.7), magnetization is sampled on a uniform rectangular mesh at points $\mathbf{x}_{\mathrm{ijk}}=\left(x_{0}+i \Delta_{x}, y_{0}+j \Delta_{y}, z_{0}+k \Delta_{z}\right)$. The computational cell has dimensions $\Delta_{x} \times \Delta_{y} \times \Delta_{z}$ and magnetization is assumed uniform inside the cell. This gives rise to artefacts at the boundaries, where the geometry of the sample is approximated as a staircase over curved surfaces. The term finite-difference rises from the direct substitution of derivatives with their discrete approximations, difference quotients:

$$
\frac{d F}{d x}\left(x_{0}\right)=\lim _{\Delta_{x} \rightarrow 0} \frac{F\left(x_{0}+\Delta_{x}\right)-F\left(x_{0}\right)}{\Delta_{x}} \simeq \frac{F\left(x_{0}+\Delta_{x}\right)-F\left(x_{0}\right)}{\Delta_{x}}
$$

for $\Delta_{x}$ small. This, of course, introduces an error in the evaluation of derivatives proportional to $\Delta_{x}$. The numerical estimation of derivative at the node $x_{i}=x_{0}+i \Delta_{x}$ can be achieved in different ways via a finite difference:

$$
\begin{array}{lr}
\overrightarrow{F\left(x_{i}\right)^{\prime}}=\frac{F\left(x_{i+1}\right)-F\left(x_{i}\right)}{\Delta_{x}} & \text { forward difference } \\
\overleftrightarrow{F\left(x_{i}\right)^{\prime}}=\frac{F\left(x_{i}\right)-F\left(x_{i-1}\right)}{\Delta_{x}} & \text { backward difference } \\
\overleftrightarrow{F\left(x_{i}\right)^{\prime}}=\frac{F\left(x_{i+\frac{1}{2}}\right)-F\left(x_{i-\frac{1}{2}}\right)}{\Delta_{x}} & \text { centered difference }
\end{array}
$$

The centered difference method gives a better approximation since its error is of second order $\overleftrightarrow{F(x)^{\prime}}-F(x)^{\prime}=o\left(\Delta_{x}^{2}\right)$, whereas the forward and backward methods are both of first order. In the same way second derivatives are approximated by second order finite differences:

$$
\overleftrightarrow{F\left(x_{i}\right)^{\prime \prime}}=\frac{F\left(x_{i+\frac{1}{2}}\right)^{\prime}-F\left(x_{i-\frac{1}{2}}\right)^{\prime}}{\Delta_{x}}
$$

By using the centered differences at $x_{i+\frac{1}{2}}$ and $x_{i-\frac{1}{2}}$ we get

$$
\overleftrightarrow{F\left(x_{i}\right)^{\prime \prime}}=\frac{F\left(x_{i+1}\right)-2 F\left(x_{i}\right)+F\left(x_{i-1}\right)}{\Delta_{x}^{2}}
$$

\subsubsection{Effective field: energy based evaluation}

When using a finite difference method to integrate eq. (1.7), the effective field needs to be evaluated at every time step at every node of the mesh. Two strategies can be used here: either compute directly the effective field at a mesh point from the magnetization (1.28) or to first compute the energy density terms (1.27) and then use eq. (1.10) to obtain the effective field value at a mesh point assuming that energy density is uniform in the cell.

In this second method the value of the field obtained is not the one at the centre of the computational cell but the averaged value across the whole cell. The second 
approach has the advantage of providing a safer approximation, since cell-averaged values permit to take into consideration large variation of field that can take place inside discretization cells, especially at boundaries for the demagnetizing field.

Using this second approach, the approximation introduced by the discretization of the problem is made on the energy calculation, from which an effective field is obtained without additional approximations. Discretized energies and associated effective fields are:

- Zeeman energy

$$
E_{z}=-\mu_{0} \int_{\Omega} \mathbf{M} \cdot \mathbf{H}_{\mathrm{a}} d V \simeq-\mu_{0} \sum_{i} \mathbf{M}\left(\mathbf{r}_{\mathrm{i}}\right) \cdot \mathbf{H}_{\mathrm{a}}\left(\mathbf{r}_{\mathrm{i}}\right) V_{i} .
$$

where $V_{i}$ is the volume of the $i$-th cell. The discretized expression for Zeeman's field derived from this approximation is simply

$$
\mathbf{H}_{\mathrm{Z}, \mathrm{i}}=\mathbf{H}_{\mathrm{a}}\left(\mathbf{r}_{\mathrm{i}}\right) .
$$

- Uniaxial anisotropy

$$
E_{K}=-\int_{\Omega} K_{u}(\mathbf{m} \cdot \hat{\mathbf{u}})^{2} d V \simeq-\sum_{i} K_{u}\left(\mathbf{m}\left(\mathbf{r}_{\mathrm{i}}\right) \cdot \hat{\mathbf{u}}\left(\mathbf{r}_{\mathrm{i}}\right)\right)^{2} V_{i} .
$$

The discretized uniaxial anisotropy field is then

$$
\mathbf{H}_{\mathrm{K}, \mathrm{i}}=\frac{2 K_{u}}{\mu_{0} M_{s}}\left(\mathbf{m}\left(\mathbf{r}_{\mathrm{i}}\right) \cdot \hat{\mathbf{u}}\left(\mathbf{r}_{\mathrm{i}}\right)\right) \hat{\mathbf{u}}\left(\mathbf{r}_{\mathrm{i}}\right) .
$$

- Exchange interaction

$$
E_{e x}=-A \int_{\Omega} \mathbf{m} \cdot\left(\frac{\partial^{2} \mathbf{m}}{\partial x^{2}}+\frac{\partial^{2} \mathbf{m}}{\partial y^{2}}+\frac{\partial^{2} \mathbf{m}}{\partial z^{2}}\right) d V
$$

where we used the identity $(\nabla f)^{2}=\nabla \cdot(f \nabla f)-f \nabla^{2} f$ for the scalar smooth functions $m_{x}, m_{y}, m_{z}$, and $\nabla \cdot(f \nabla f)$ term is responsible for the standard boundary conditions rising from Brown's equation (1.9).

Here the discretization is not trivial but becomes straightforward once we use finite differences in place of derivatives:

$$
E_{e x} \simeq-A \sum_{i} V_{i} \mathbf{m}\left(\mathbf{r}_{\mathrm{i}}\right) \cdot\left(\sum_{j=x, y, z} \delta_{j}^{2} \mathbf{m}\left(\mathbf{r}_{\mathrm{i}}\right)\right)
$$

where

$$
\delta_{j}^{2} \mathbf{m}\left(\mathbf{r}_{\mathrm{i}}\right)=\frac{\mathbf{m}\left(\mathbf{r}_{\mathrm{i}}+\Delta_{j}\right)-2 \mathbf{m}\left(\mathbf{r}_{\mathrm{i}}\right)+\mathbf{m}\left(\mathbf{r}_{\mathrm{i}}-\Delta_{j}\right)}{\Delta_{j}^{2}} .
$$


The discretized exchange field is then

$$
\mathbf{H}_{\mathrm{ex}, \mathrm{i}}=\frac{2 A}{\mu_{0} M_{s}} \sum_{j=x, y, z} \frac{\mathbf{m}\left(\mathbf{r}_{\mathrm{i}}+\Delta_{j}\right)-2 \mathbf{m}\left(\mathbf{r}_{\mathrm{i}}\right)+\mathbf{m}\left(\mathbf{r}_{\mathrm{i}}-\Delta_{j}\right)}{\Delta_{j}^{2}} .
$$

It only remains to express the demagnetizing energy and associated field in discretized form. This operation requires additional accuracy, since magnetostatic interaction, being long range, is the result of the sum of a number of interactions. A small error in the numerical description of such interaction can yield large errors. Moreover, the estimation of the demagnetizing field is, by far, the most time-consuming part of micromagnetic simulations, so that numerical strategies need to be applied to reduce the computational weight of this step.

The next section is dedicated to describe the strategy used to compute in an accurate yet fast way the demagnetizing field.

\subsubsection{Demagnetizing field}

It has been shown how the magnetostatic field is the gradient of a scalar potential $\Phi$ (1.19). Let's consider a finite volume $\Omega^{\prime}$ uniformly magnetized $\mathbf{M}=\mathbf{M}_{\Omega^{\prime}}$. The divergence of $\mathbf{M}$ is zero inside $\Omega^{\prime}$ and

$$
\Phi(\mathbf{r})=\frac{1}{4 \pi} \mathbf{M}_{\Omega^{\prime}} \cdot \int_{\partial \Omega^{\prime}} \frac{d \mathbf{S}^{\prime}}{\left|\mathbf{r}-\mathbf{r}^{\prime}\right|}
$$

The dipolar field generated by the magnetized volume $\Omega^{\prime}$ at the point $\mathbf{r}$ in space is

$$
\mathbf{H}_{\mathrm{d}}^{\Omega^{\prime}}(\mathbf{r})=-\nabla \Phi(\mathbf{r})=-\frac{1}{4 \pi} \mathbf{M}_{\Omega^{\prime}} \cdot \nabla \int_{\partial \Omega^{\prime}} \frac{d \mathbf{S}^{\prime}}{\left|\mathbf{r}-\mathbf{r}^{\prime}\right|} .
$$

Let's take now a finite volume $\Omega$ around $\mathbf{r}$. The average dipolar field over $\Omega$ is

$$
\left\langle\mathbf{H}_{\mathrm{d}}^{\Omega^{\prime}}\right\rangle_{\Omega}=\frac{1}{\Omega} \int_{\Omega} \mathbf{H}_{\mathrm{d}}^{\Omega^{\prime}}(\mathbf{r}) d r^{3}
$$

which can be rewritten as

$$
\left\langle\mathbf{H}_{\mathrm{d}}^{\Omega^{\prime}}\right\rangle_{\Omega}=-\frac{1}{4 \pi \Omega} \mathbf{M}_{\Omega^{\prime}} \cdot \int_{\Omega} \nabla\left(\int_{\partial \Omega^{\prime}} \frac{d \mathbf{S}^{\prime}}{\left|\mathbf{r}-\mathbf{r}^{\prime}\right|}\right) d r^{3} .
$$

This can be shortened as

$$
\left\langle\mathbf{H}_{\mathrm{d}}^{\Omega^{\prime}}\right\rangle_{\Omega}=-\mathbf{M}_{\Omega^{\prime}} \cdot \overline{\mathbf{N}}
$$

where the tensor $\overline{\mathbf{N}}$ is given by

$$
\overline{\mathbf{N}}=\frac{1}{4 \pi \Omega} \int_{\Omega} \nabla\left(\int_{\partial \Omega^{\prime}} \frac{d \mathbf{S}^{\prime}}{\left|\mathbf{r}-\mathbf{r}^{\prime}\right|}\right) d r^{3}=\frac{1}{4 \pi \Omega} \int_{\partial \Omega} d \mathbf{S} \int_{\partial \Omega^{\prime}} \frac{d \mathbf{S}^{\prime}}{\left|\mathbf{r}-\mathbf{r}^{\prime}\right|}
$$


where $d \mathbf{S}=\mathbf{n} d S, \mathbf{n}$ being the versor normal to the surface, and we have used divergence theorem to pass from volume to surface integral [33].

We now consider a non uniform magnetization distribution. As considered in (1.1.3), in the micromagnetics framework, it is correct to represent the magnetization or magnetic field at a point as the average of magnetic moments in a small volume around that point [2]

$$
\mathbf{M}(\mathbf{r})=\frac{1}{\Omega(\mathbf{r})} \sum_{\boldsymbol{\mu} \in \Omega(\mathbf{r})} \boldsymbol{\mu},
$$

where the dimensions of the volume are smaller than the exchange length 1.3.2 and the Bloch length 1.3.2. In the numerical finite-difference approximation of the problem, the magnetized sample is discretized in rectangular-shaped volumes centered at the mesh points, while magnetization is uniform inside each cell. If we consider only two distinct cells $i$ and $j$ the magnetic field generated by magnetization at cell $j$ averaged over cell $i$ is $\mathbf{H}_{\mathrm{d}, \mathrm{i}}=-\overline{\mathbf{N}}_{\mathrm{i}, \mathrm{j}} \cdot \mathbf{M}_{\mathbf{j}}$. If we now consider the whole sample, average dipolar field at cell $i$ is the sum of the contributions from all other cells, including cell $i$ itself.

$$
\mathbf{H}_{\mathrm{d}, \mathrm{i}}=-\sum_{j} \overline{\mathbf{N}}_{\mathrm{i}, \mathrm{j}} \cdot \mathbf{M}_{\mathrm{j}}
$$

where

$$
\overline{\mathbf{N}_{\mathrm{i}, \mathrm{j}}}=\frac{1}{4 \pi \Omega_{i}} \int_{S_{i}} d \mathbf{S} \int_{S_{j}} \frac{d \mathbf{S}^{\prime}}{\left|\mathbf{r}-\mathbf{r}^{\prime}\right|}
$$

and we refer to the surface boundary as $S$ instead of $\partial \Omega$. As can be observed from its expression, the demagnetizing tensor is symmetric $\overline{\mathbf{N}}_{\mathrm{i}, \mathrm{j}}=\overline{\mathbf{N}}_{\mathrm{j}, \mathrm{i}}$. Moreover, the value of the demagnetizing tensor for two cells only depends on their relative position and shape [33]. The exact value of such integrals is calculated at the beginning of a numerical simulation.

From what has been shown so far, the computation of $\mathbf{H}_{\mathrm{d}}$ requires $O\left(N^{2}\right)$ operations for $N$ computational cells, since the evaluation of the magnetostatic field at every cell $i$ requires the multiplication of magnetization at each cell $j$ by the demagnetizing tensor $\overline{\mathbf{N}}_{\mathrm{i}, \mathrm{j}}$ (2.2). However, as anticipated at the beginning of the chapter, equispaced meshing allows us to exploit the Fast Fourier Transform (FFT) algorithm [25] bringing the computational effort down to $O(N \log N)$ operations. This is possible because equation (2.2) can be recognized as a discrete convolution, which corresponds to multiplication in Fourier's space:

$$
\mathcal{F}\left(\mathbf{H}_{\mathrm{d}, \mathrm{i}}\right)=\mathcal{F}\left(\overline{\mathbf{N}}_{\mathrm{i}, \mathrm{j}}\right) \cdot \mathcal{F}\left(\mathbf{M}_{\mathrm{j}}\right)
$$

However, convolution computed via FFT assumes that input repeats periodically, while magnetization over a finite sample is not. To avoid artifacts from FFT computation, a so-called zero padding technique need to be performed along all three dimensions of our sample [25]. This increases by a factor of eight the computational effort. 


\subsubsection{Thermal field}

Thermal fluctuation in a magnetic system can be described by including a random noise in the deterministic dynamic equation (1.6), turning it into a stochastic equation. Brown showed [34] what are the statistical properties of the random field to be included in order to reproduce correctly the equilibrium thermodynamic of a single domain particle. Although this does not apply directly to a spatially varying magnetic distribution, the discretized system studied via micromagnetic simulations can be formally regarded as an assembly of single-domain interacting particles [14] and, therefore, Brown's formalism can be applied.

A random thermal field $\mathbf{H}_{\mathrm{th}}$ is added to the effective field, having the properties

$$
\begin{aligned}
\left\langle\mathbf{H}_{\mathrm{th}}^{\alpha, i}(t)\right\rangle & =0 \\
\left\langle\mathbf{H}_{\mathrm{th}}^{\alpha, i}(t) \mathbf{H}_{\mathrm{th}}^{\beta, j}\left(t^{\prime}\right)\right\rangle & =2 D \delta_{i j} \delta_{\alpha \beta} \delta\left(t-t^{\prime}\right) .
\end{aligned}
$$

meaning that a random value is assigned to each Cartesian component of $\mathbf{H}_{\mathrm{th}}$ at each computational cell. Such random values follow a Gaussian distribution with zero mean value, and variance $2 D$, uncorrelated in space $\delta_{i j}$, time $\delta\left(t-t^{\prime}\right)$ and component-wise $\delta_{\alpha \beta}$. The coefficient $D$ is determined by imposing that the stationary solution of the stochastic equation satisfies Maxwell-Boltzmann statistics [35] when thermodynamic equilibrium is reached

$$
D=\frac{\alpha k_{B} T}{\left(1+\alpha^{2}\right) \gamma_{0} \mu_{0} M_{s} V}
$$

Here, $\alpha$ is Gilbert's damping parameter, $k_{B}$ is Boltzmann constant, $T$ the system's temperature and $V$ the micromagnetic cell volume. The added field has the form:

$$
\mathbf{H}_{\mathrm{th}, \mathrm{i}}=\mathbf{N}_{\mathrm{i}}(t) \sqrt{\frac{2 D}{\Delta_{t}}}
$$

where $\mathbf{N}_{\mathrm{i}}(t)$ is a stochastic vector whose components follow a standard normal distribution and $\Delta_{t}$ the discrete time step of the numerical integration.

\subsubsection{Workflow}

A micromagnetic software is usually comprised of two main runtime steps as shown in fig. 2.1. The first part takes care of the initialization process: reading input from a file, from a graphical user interface or directly from variables initialized inside the executable itself, the dimensions of the sample, the discretization mesh size, the material parameters and the initial magnetization are defined. In this first step also the demagnetizing tensor is computed and stored in memory for later usage. Moreover, all the simulation parameters, such as error tolerance, stopping criteria for the simulation, time step, output writing directives need to be specified at this time. The 
second part is dedicated to the integration of the differential equation (1.7) describing magnetization dynamics using an iterative numerical method. The time span of integration, the stopping conditions and output saving parameters specified earlier on affect the duration of the simulation. Since the operations performed in the definition and initialization step are only called once per simulation, the focus can be fully oriented on the accuracy of calculations, neglecting the computing performance. In the time iteration step, on the other hand, operations and routines are repeated thousands of times and efficiency is a fundamental parameter.

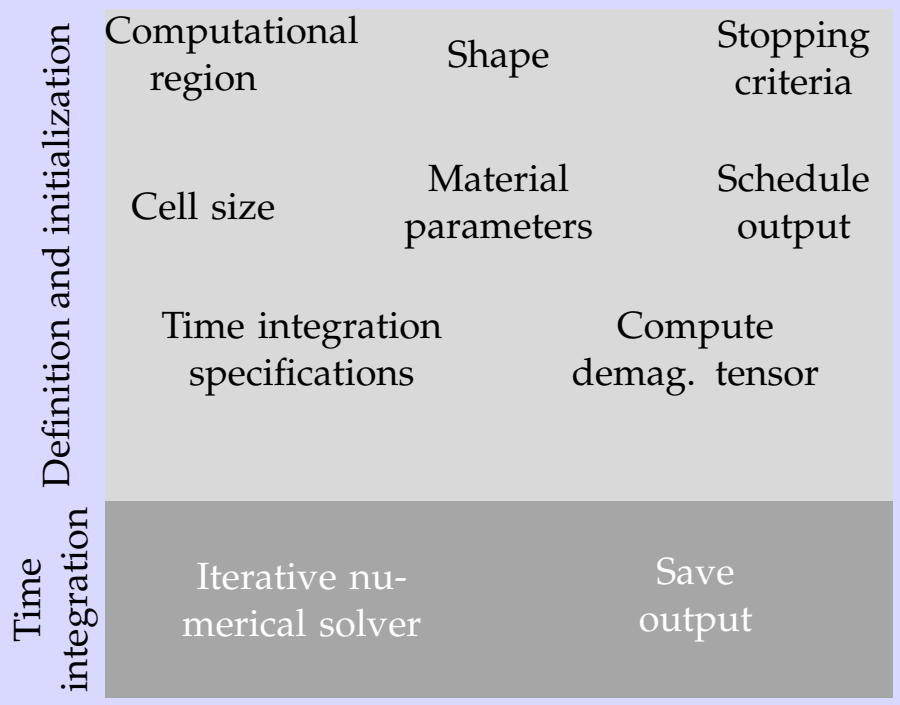

FIGURE 2.1: Division of the two parts of runtime processing of a micromagnetic solver: light gray upper part is the initialization stage, where operations are performed once and performance is not important. Lower part is the iterative stage, where iterative methods are applied to numerically evaluate the dynamics.

The time integration step follows the algorithm schematically represented in fig. 2.2. Unless the conditions for stopping the numerical integration are true (maximum torque smaller than $\epsilon$, maximum number of iterations reached, stopping time reached ) the effective field is computed and the magnetization is updated. Output from the simulation can be saved at every time-step, periodically, to save memory, or only at the end of the simulation, if we are only interested in the final state. The computation of the effective field, presented in 2.3, is the most time-consuming part.

As can be observed in fig. 2.3, the computation of the effective field contains the evaluation of the demagnetizing field, where the FFT algorithm is used twice to transform convolution in point-wise product in Fourier space, as discussed in subsection 2.1.3. It is important to note that the demagnetizing tensor is only needed in its transformed form $\mathcal{F}(\overline{\mathbf{N}})$. It is useful then to compute it only once at the initialization step and store the transformed version of the tensor. 


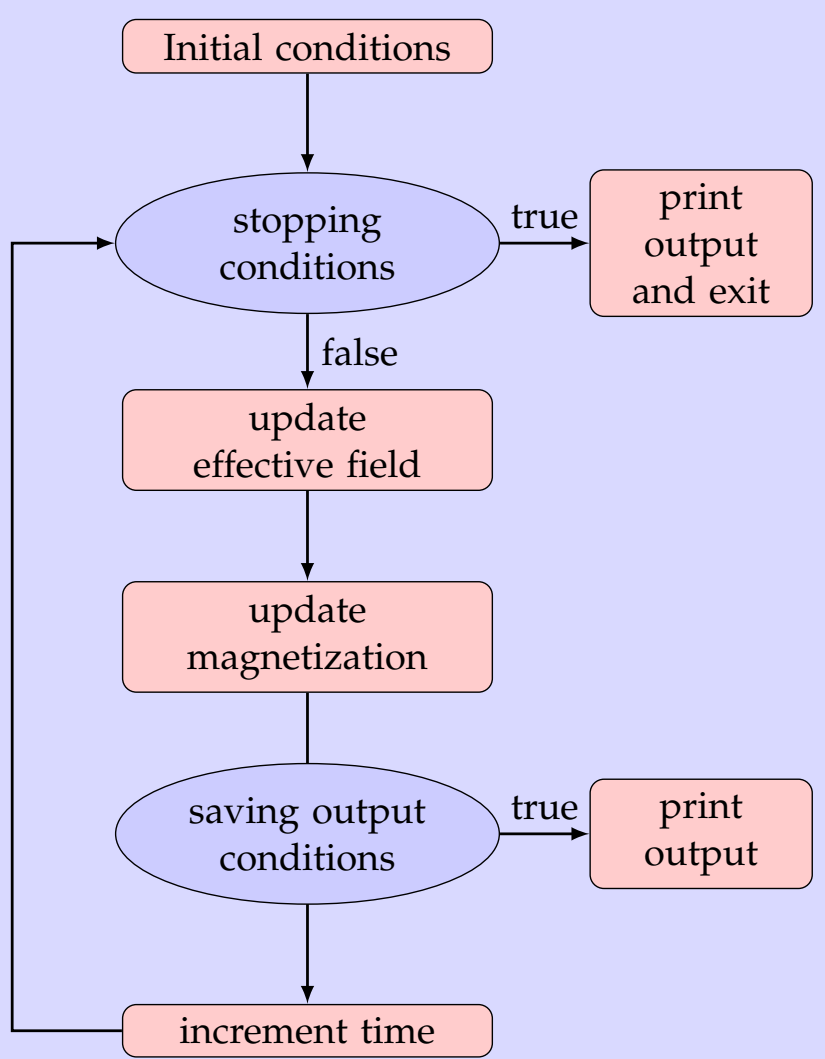

FIGURE 2.2: Schematic of the global workflow of the iterative stage of a micromagnetic solver.

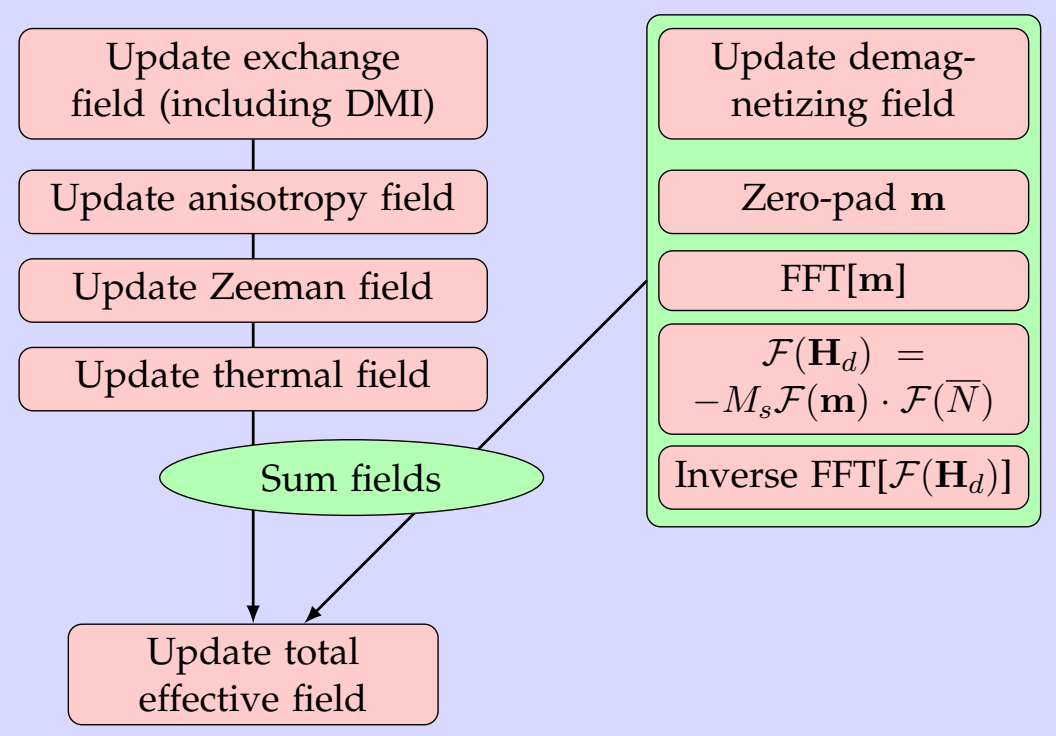

FIGURE 2.3: Schematic representation of effective field computation. Demagnetizing field computation is by far the most computationally demanding step. 


\subsubsection{Time integration}

The core of a numerical micromagnetic solver is the time-integrator engine, the algorithm that computes the magnetization distribution at successive instants of time. Many different algorithms exist to find numerical solutions of systems of differential equations with given initial values [36]. In our code, as it is done in some of the most popular solvers (OOMMF [27], MuMax3 [28]), explicit Runge-Kutta methods have been used: fixed step fourth order method (RK4) and adaptive time step method of order $5[37,38]$ (RK5(4)7FC). In what follows we briefly describe these methods.

Let's represent our initial values problem as

$$
\begin{aligned}
\frac{d \mathbf{m}}{d t}(t) & =f(t, \mathbf{m}(t)), \\
\mathbf{m}\left(t_{0}\right) & =\mathbf{m}_{0} .
\end{aligned}
$$

If $\mathbf{m}_{\mathrm{n}}=\mathbf{m}\left(t_{n}\right)$ is the exact solution at the instant of time $t_{n}=t_{0}+n \Delta_{t}$, an approximated solution $\tilde{\mathbf{m}}_{\mathrm{n}+1}$ of $\mathbf{m}_{\mathrm{n}+1}=\mathbf{m}\left(t_{n+1}\right)$ is given by

$$
\tilde{\mathbf{m}}_{\mathrm{n}+1}=\mathbf{m}_{\mathrm{n}}+\sum_{i=1}^{s} b_{i} \mathbf{k} i,
$$

where

$$
\begin{aligned}
& \mathbf{k}_{1}=\Delta_{t} f\left(t, \mathbf{m}_{\mathrm{n}}\right) \\
& \mathbf{k}_{\mathrm{i}}=\Delta_{t} f\left(t+c_{i} \Delta_{t}, \mathbf{m}_{\mathrm{n}}+\sum_{j=1}^{i-1} a_{i j} \mathbf{k}_{\mathrm{j}}\right), \quad i=2,3, \ldots, s .
\end{aligned}
$$

Runge-Kutta methods are improved versions of Taylor's methods [36], they keep the high order local truncation error, without requiring the evaluation of the higher derivatives of the function required by Taylor's methods: approximation at time $t_{n+1}=t_{n}+\Delta_{t}$ is performed by evaluating approximations $\mathbf{k}_{\mathrm{i}}$ of the increment at intermediate steps $t_{n}+c_{i} \Delta_{t}$ (2.1.6) and then taking a particular weighted average of such intermediate approximations to approximate the increment at time $t_{n+1}$ (2.1.6). A Runge-Kutta method is fully characterized by its Butcher's tableau [39] where the coefficients $a_{i j}, b_{i}$ and $c_{i}$ are explicitly written.

The Butcher tableau for RK4 and RK5(4)7FC are shown in table 2.1.

The RK5(4)7FC algorithm [38] has several advantages. Even though it requires seven evaluations of the effective field per time step, it offers much higher accuracy and stability compared to lower order methods. This, combined with its adaptive time step properties, translates into a much larger time step for integration of (1.7) that can considerably speed up simulations while keeping high accuracy. It also has the First Same As Last (FSAL) property [37], meaning that in case of acceptance of the previous step evaluation, the first coefficient $\mathbf{k}_{1}$ is already computed as the $\mathbf{k}_{7}$ of the previous step. This feature reduces the effective field evaluations per iteration to 


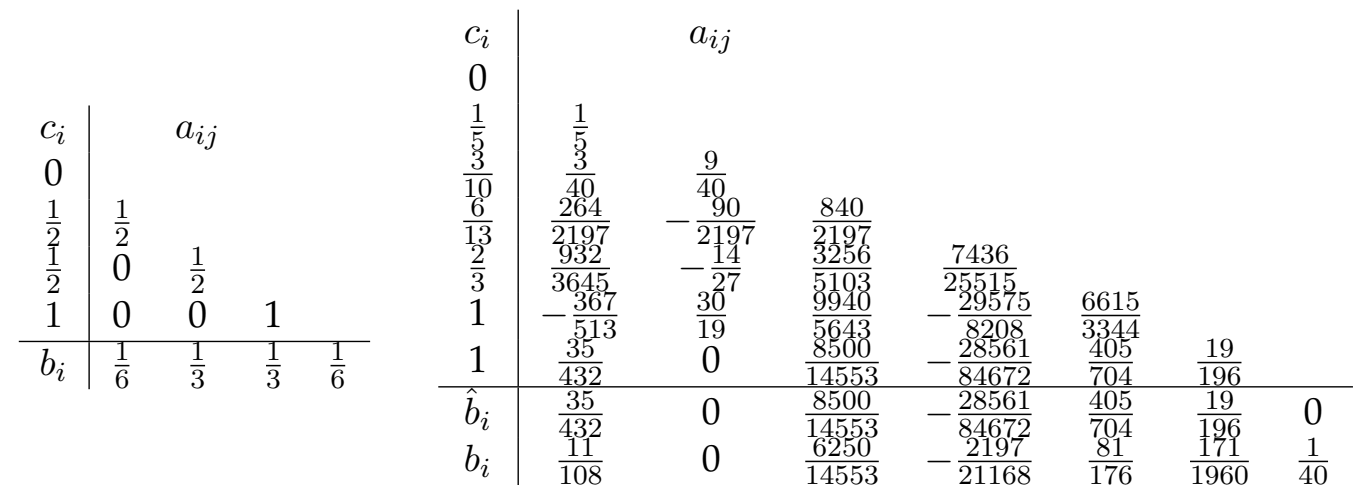

TABLE 2.1: Butcher tableau for Runge-Kutta fourth order solver (left) and for Dormand-Prince fifth order solver.

six. Other two versions of RK5(4)7 are implemented, RK5(4)7FM, default in Mumax3 and OOMMF, which has higher accuracy but lower stability, and RK5(4)7FS, which offers larger stability at the cost of lower accuracy.

The remarkable feature of an adaptive solver such as RK5(4)7FC appears in its Butcher's tableau, where two different $b_{i}$ coefficients are shown. In fact, with the computed $\mathbf{k}_{\mathrm{i}}$, weighted in the two ways, $\hat{b}_{i}$ and $b_{i}$, two different approximations of the solution can be obtained and their difference can be used to estimate the error of the given step. This on-the-run error estimation allows the solver to increase the time step for the subsequent iterations if the estimated error is below a given precision, or to reject the approximated solution and recompute it with a smaller time step. Such procedure has two main benefits: on one hand, the time step for slow dynamics simulations tends to stabilize at the maximum time step allowed by the numerical precision, on the other, in the case of studies where large abrupt variations of magnetizations take place during dynamics, for example a large pulsed field at some instant of time, there is no need to worry about the choice of the time step. Where the dynamics becomes more irregular, thus requiring a smaller time step for numerical integration, the solver will automatically adjust the time step to take care of it.

\subsubsection{Code verification}

Even though micromagnetic theory was well established by the early 1960's [2], the computational efforts required to simulate even simple structures were too demanding. Since the late 1980's, when computers became fast enough, micromagnetic simulations have become an important tool in research and a number of different groups over the world developed their own micromagnetic code. Efforts were made then to unify and standardize strategies and to test the validity of different micromagnetic codes, both in the numerical precision and in the capability to reproduce adequately the physics. To this aim a series of so called standard problems have been designed and proposed to the numerical micromagnetic community by the $\mu \mathrm{MAG}$ - Micromagnetic Modeling Activity Group. In particular, the Standard problem \#4 allows 
us to test the accuracy of a micromagnetic code in describing complex magnetization dynamics. The specifications of this problem are the following. A magnetic platelet of permalloy material parameters and dimension $500 \times 125 \times 3 \mathrm{~nm}^{3}$ is at equilibrium in the so called S-state shown in fig. 2.4, obtained by by slowly relaxing an initial off-equilibrium uniform configuration.

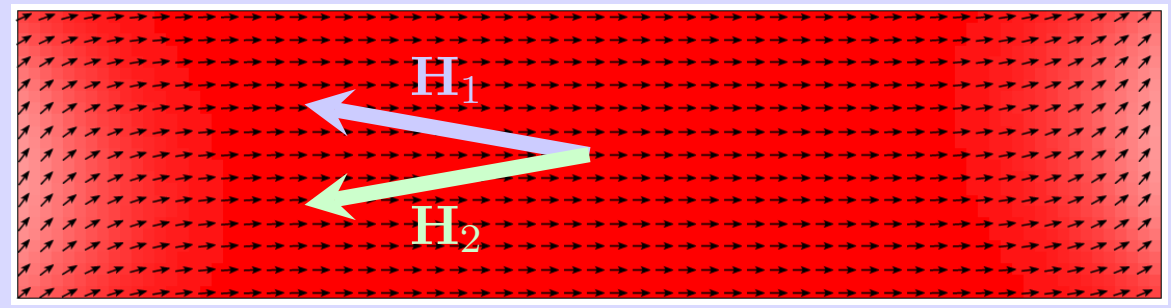

FIGURE 2.4: S-state initial configuration for standard problem \#4 black arrows show in-plane magnetization orientation. The two light color arrows represent the external fields $\mathbf{H}_{1}=25 \mathrm{mT}$ and $\mathbf{H}_{2}=36 \mathrm{mT}$ applied to the equilibrium configuration.

Subsequently, magnetization orientation is switched by the application of two different external fields, each one producing a different dynamical evolution during reversal. The variation of the averaged components of magnetization is monitored in time and it is used to control the correct time evolution of the system, together with snapshots of magnetization at precise instants of time. On the $\mu \mathrm{MAG}$ website, a number of solutions have been submitted by different groups and it is hence possible to benchmark one's code by comparing the own solution against other well established micromagnetic solvers. Figure 2.5 shows the comparison of the solution obtained with our code with the one obtained by M. J. Donahue and D. G. Porter in 2014 with the double precision open source software OOMMF [27], where they use full precision and discretize the sample in $1 \mathrm{~nm}$ sized cubic cells.

The agreement is good and the two solutions appear to describe the same reversal dynamics. The ultimate test is the quantitative comparison of the $\langle\mathbf{m}\rangle_{y}$ time evolution. As can be observed in fig. 2.6, the solution with a larger cell size presents numerical discrepancies due to the different discretization while describing the same physics. It is in fact important to note that reducing the cell size the solution converges to the reference one. One last control is the comparison of a snapshot of magnetization taken when $\langle\mathbf{m}\rangle_{x}=0$. As shown in figure 2.5, even the coarser discretization used gives a consistent result.

\subsection{Parallel computing}

This section is devoted to the presentation of parallel computing performed on Graphic Processor Units (GPU) with the programming language CUDA ( Compute Unified Device Architecture), a general purpose parallel computing language that allows the 

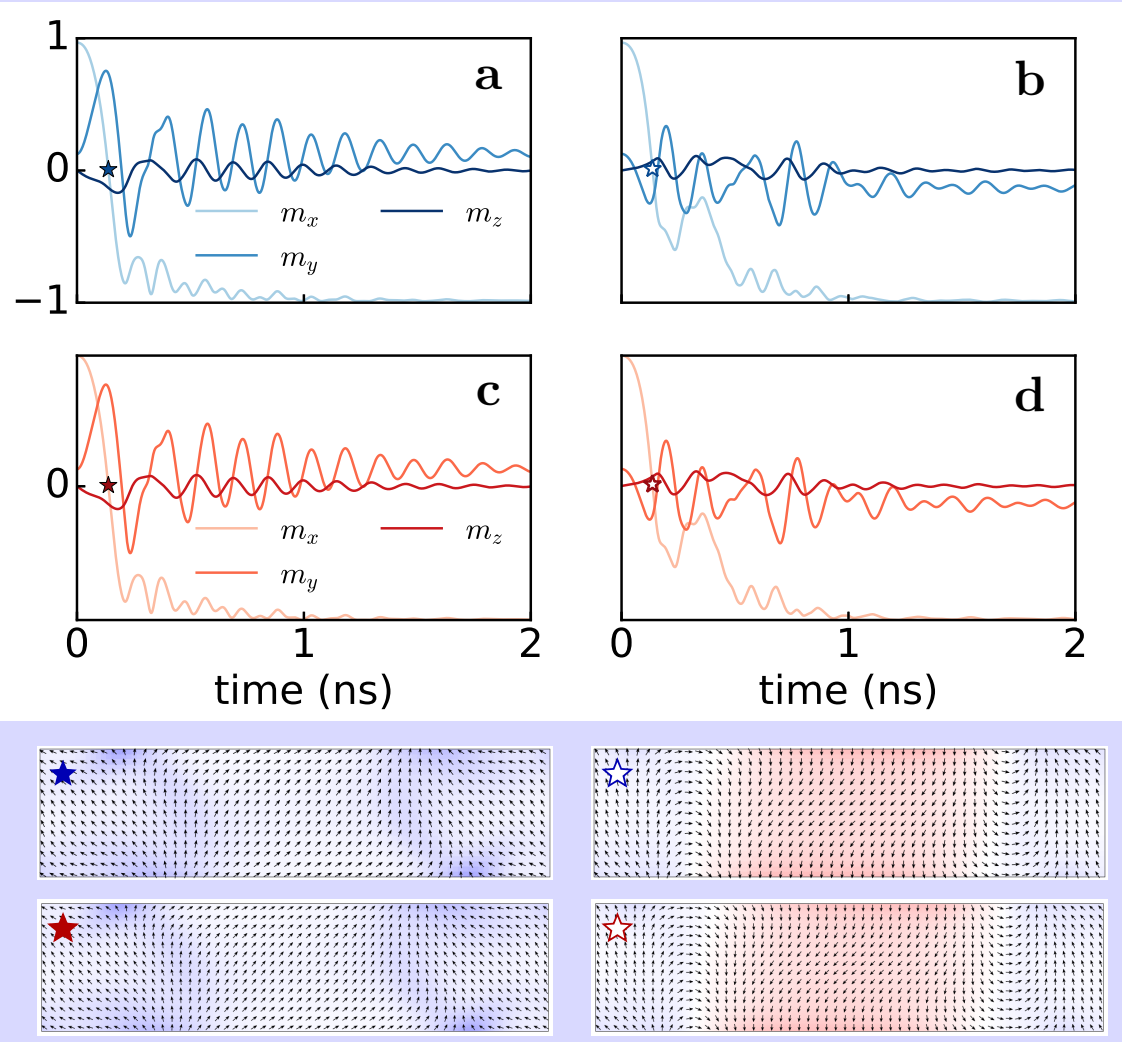

FIGURE 2.5: Average magnetization evolution for the applied field $\mathbf{H}_{1}$ ( $\mathbf{a}$ and $\mathbf{c}$ ) and $\mathbf{H}_{2}$ ( $\mathbf{b}$ and $\mathbf{d}$ ). In $\mathbf{a}$ and $\mathbf{b}$ the soluion provided by our code with mesh size $\Delta_{x}=\Delta_{y}=5 \mathrm{~nm}$ and $\Delta_{z}=3 \mathrm{~nm}$. In $\mathrm{c}$ and $\mathbf{d}$ the solution submitted by Donahue and Porter on $\mu \mathrm{MAG}$ website. The corresponding snapshots of magnetization taken when $\langle\mathbf{m}\rangle_{x}=0$ are shown below for comparison.

programmer to control the parallel compute engine in NVIDIA GPUs to solve complex computational problems in a more efficient way than on a central processing unit (CPU) [40]. CUDA comes with a software environment that allows developers to use $\mathrm{C} / \mathrm{C}++$ as a high-level programming language.

\subsubsection{Hardware architecture}

Being designed with the purpose of intensive computations required for graphics rendering, GPUs are particularly suitable for performing a large number of operations in parallel, dedicating more transistors to logic and arithmetic operations than to flow control and to cache.

From the CUDA programming guide, it is possible to check how nowadays graphic cards outperform state of the art CPU processors in terms of number of 


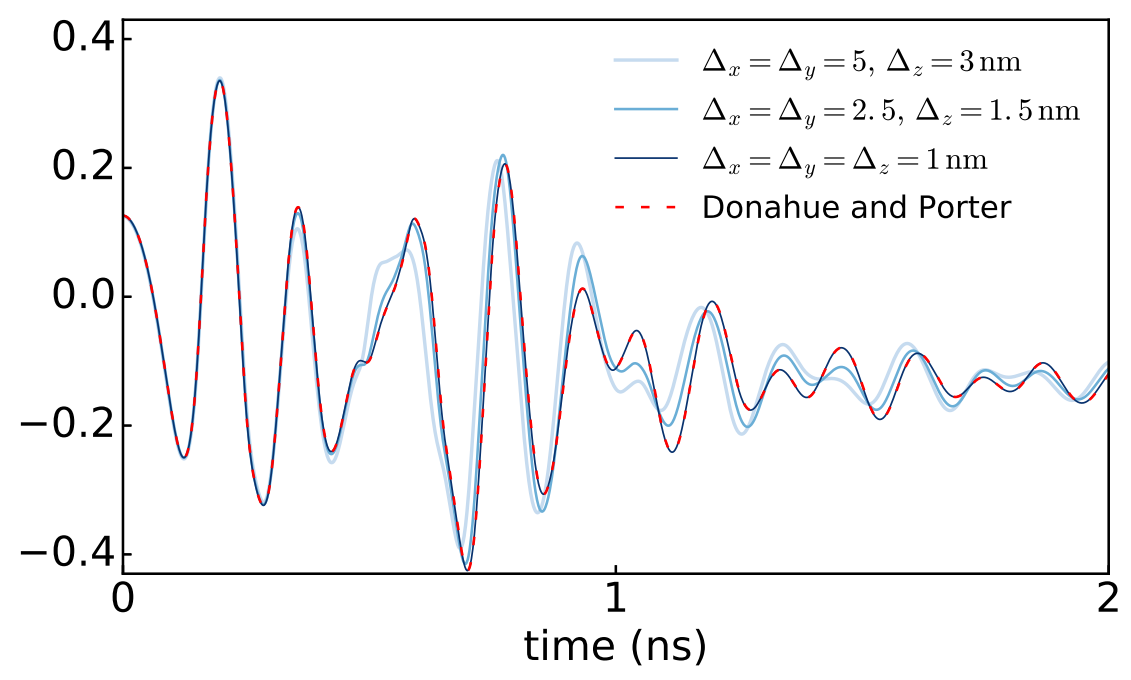

FIGURE 2.6: Evolution of $\langle\mathbf{m}\rangle_{y}$ for the second applied field. Different discretization mesh sizes are compared with the solution of Donahue and Porter.

floating-point operations per second and memory bandwidth ${ }^{1}$. Moreover, processors technology has reached a plateau in terms of single threaded clock speed and is turning to multi-thread, multi-core processing [41]. This means that, with adequate programming technique, the computational power of a tabletop desktop computer with a couple of good graphic cards can be in the order of a small computer cluster, while being much easier to handle, to maintain and much cheaper.

\subsubsection{GPU for scientific computing}

It is desirable that a software dedicated mostly to mathematical calculations like a micromagnetic solver makes use of GPU computational power. In order to do so the software must be designed in such a way that the CPU that handles the sequence of operations can order the GPU to perform packets of computational work. A specific programming language has to be used to interact with GPUs: CUDA, developed by NVIDIA, and Stream SDK, by AMD, have been developed to be used on their respective graphic cards. OpenCL, on the other hand, is a versatile open source framework for parallel computing, being platform independent. The language used in programming our software is CUDA, since NVIDIA has been pioneering this branch of software engineering, specific packages and optimized libraries are available for developers. With CUDA we are able to allocate memory on the GPU, copy data back and forth between GPU (Device) and CPU (Host) and, of course, define functions that perform operations on GPU. These functions are called Kernels. A kernel

\footnotetext{
${ }^{1}$ the rate at which data can be read from or stored into memory
} 
is called from the CPU and upon call the number of parallel threads that will simultaneously perform the operation is defined. Concurrent threads are organized in Blocks and blocks are organized in a grid. The dimension of blocks and grid are specified at the kernel call. The main bottleneck of this kind of software is the transfer of memory between host and device, so that for best overall performance it is crucial to minimize data transfer between host and device. Sometimes copying to host is mandatory, since certain operations, such as printing output to file, cannot be performed by GPU. When writing parallel code to solve a given problem, the ratio between computation and communication between host and device is called granularity [14]. In a coarse granularity program, large portions of code are executed without any communication between CPU and GPU, so that the power of GPU computing can be exploited avoiding the data transfer bottleneck.

\subsubsection{A parallel computing example: Voronoi tessellation}

The simplest speed up provided by parallel computing that can be imagined is the conversion of an operation iterated over an array to a single operation performed by a kernel launch over the whole array. This results in a considerable speed-up for a finite difference solver where the same differential equation needs to be timeintegrated over an array of mesh points. Faster computation is achieved in many different routines whose translation from serial to parallel code is not always straightforward. Voronoi tessellation of plane serves as a good example of a non-standard routine that benefits from being translated to GPU code. It also introduces some physics into a section that has been devoted exclusively to computer and software engineering so far.

Many magnetic materials present a polycrystalline structure, meaning that the crystalline structure is not homogeneous but varies in different regions. This can happen during a sputtering deposition process, when the first atoms of the material deposit on the substrate at isolated random locations, serving as seeds for the subsequent atoms that stick to the seeds. This process naturally forms a Voronoi tessellation of the deposited magnetic film. The tessellation of plane is obtained by placing a number of points (seeds) in the plane at random positions, then dividing the plane in polygons formed by the points that are closer to the seed they contain than to others. In this way the polygon edges are segments of the axis between two seeds. Voronoi tessellation appears naturally when structures grow simultaneously from different points filling space and it is ubiquitous in nature: tissue cells, territories of animals, fur and shell patterns (giraffe, turtle...), crystals and grains growth, cracks in dried mud. Voronoi tessellation is henceforth extensively used in many branches of science from biology to archaeology to computer science.

A standard algorithm to realize a Voronoi tessellation of plane with a serial code has $O\left(N^{2}\right)$ time complexity in number of seeds $N$, while an efficient algorithm is Fortune's Algorithm [42], which has $O(N \log N)$ complexity. 


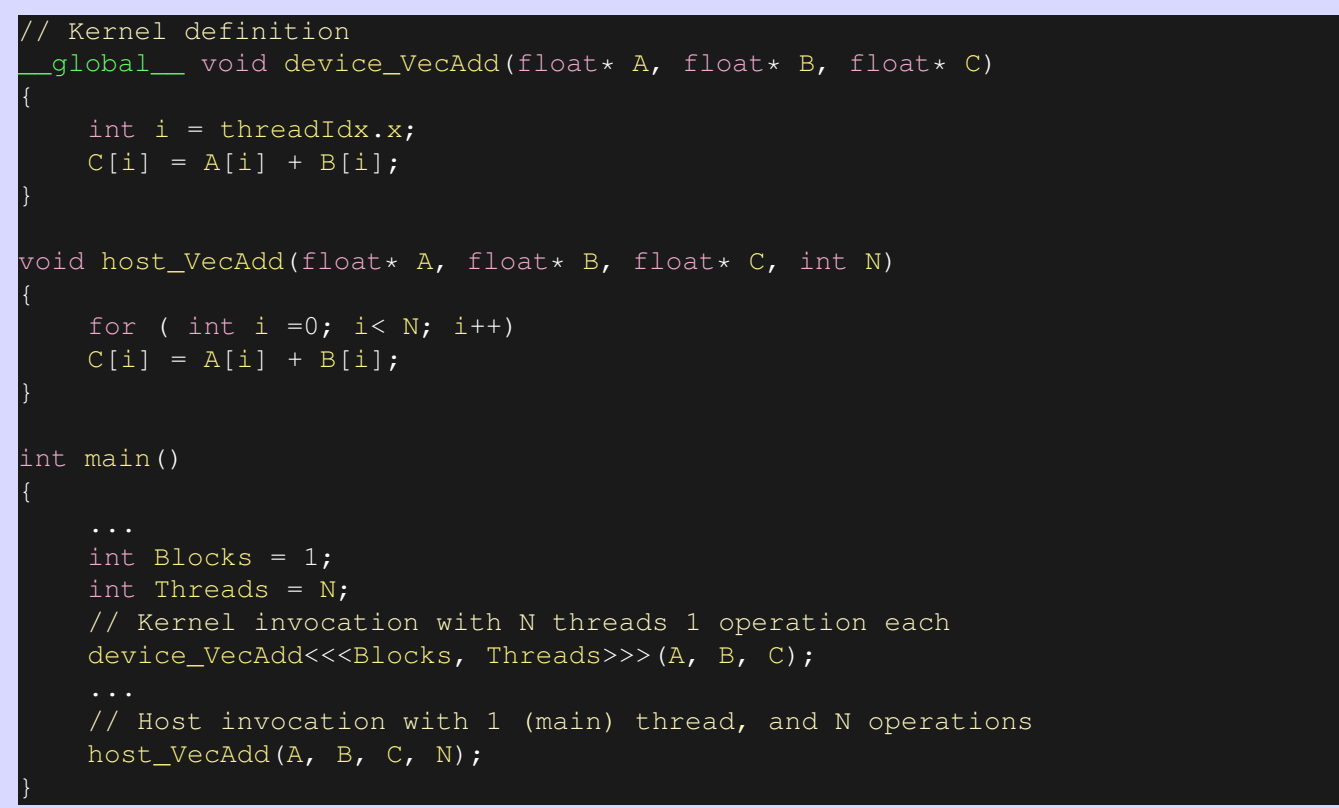

FIGURE 2.7: CUDA basic kernel example. A kernel is defined as a void function with the _ global__ keyword. A kernel call asks for the number of blocks in the grid and the number of threads per block.

A parallel algorithm to compute Voronoi tessellation of a finite plane is the flooding algorithm: it considers each seed as a color source and expands the corresponding coloured region radially until a neighbouring Voronoi cell is met. Such algorithm complexity is linear in the average radius of the Voronoi cells, which is $r=$ $\sqrt{S / \pi N_{\text {seeds }}}$, where $S$ is the surface that we want to tessellate. An improvement of such algorithm is the so called jump flooding algorithm (JFA) $[43,44]$ where instead of expanding the region surrounding each seed, we jump from the cell where the seed is by a step $\Delta=n / 2^{k}$. We reach the eight cells given by $(x+i, y+j)$ where $i, j=\{-\Delta, 0, \Delta\}$ and colour it of the seed's color if it is not coloured yet. If it already has another seed's color we check which of the two seeds is closer to the cell under consideration and assign that color. This algorithm completes the tessellation in $\log _{2} n$ steps, $n$ being the number of cells along the largest dimension of the sample. The two processes are compared in fig. 2.8. The code used to generate the two dimensional Voronoi tessellation is presented in the Appendix A. 

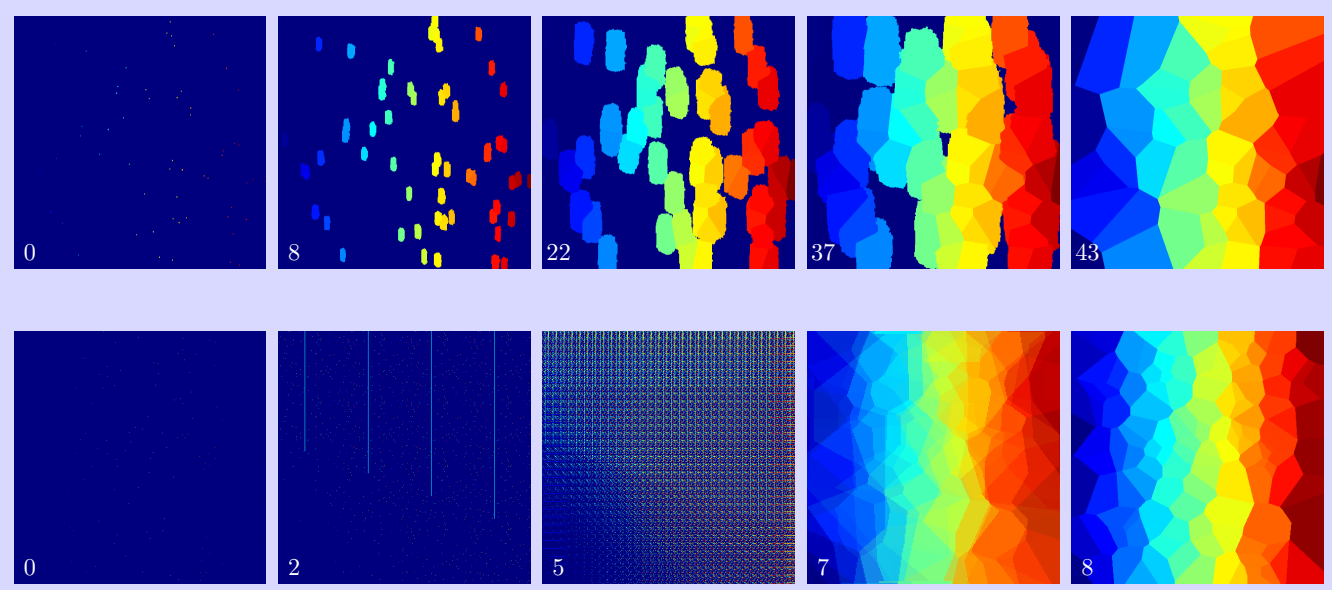

FIGURE 2.8: Generation of Voronoi tessellation using parallel computing. Flooding algorithm (top row) completes the task in $O(r)$ steps where $r$ is the average radius of the Voronoi cell. Jump flooding algorithm (bottom row) takes $O\left(\log _{2} n\right)$ steps, where $n$ is the maximum lateral size of the computational region. 


\section{Chapter 3}

\section{Domain wall dynamics in thin films with disorder}

\subsection{Introduction}

\subsubsection{General context}

This chapter is devoted to the the study of field-driven domain wall dynamics in CoFeB thin films. Magnetic domain wall (DW) dynamics in thin films and nanostrips have become the subject of renewed interest over the last years because of recently proposed applications in data storage [45, 46], logic [8] and sensing devices [5] based on controlled motion of domain walls. In addition, domain wall dynamics can play an important role in the switching process of magnetic tunnel junction cells, one of the most relevant technological application of spintronics [47]. For such applications, ultrathin films with high perpendicular magnetic anisotropy (PMA) present advantages over in-plane magnetized materials, such as narrower domain walls and better scalability. Among the materials with high PMA being currently explored, $\mathrm{CoFeB}$ thin films sandwiched between heavy metals such as Ta or Pt and oxides like $\mathrm{AlO}_{\mathrm{x}}$ or $\mathrm{MgO}$ are one of the most promising ones [21], since they exhibit low depinning fields [9] and reasonably low damping [48] while maintaining the relevant ferromagnetic properties useful for applications.

The success of domain wall based applications, however, strongly relies on the ability to move domain walls in a predictable and controllable way. To achieve such degree of control, it is necessary to understand how disorder in manufactured thin films (structural defects, grain boundaries, edge roughness, inhomogeneities at the interface with substrates and thickness fluctuations) affects domain wall motion.

The main features of field-driven domain wall dynamics in ferromagnetic strips have been presented in chapter 1 and are well captured by the one dimensional model of Walker and Slonczewski $[49,50]$. We recall that this model predicts a velocity field dependence characterized by two main regimes: the linear steady regime below the Walker field $H_{a}<H_{W}$, where the DW moves rigidly and velocity is inversely proportional to Gilbert's damping parameter $\alpha$, and the linear precessional regime [51, 13], where the mobility is proportional to $\left(\alpha+\alpha^{-1}\right)^{-1}$. These two linear 
regimes are separated by an intermediate regime after Walker field, where velocity shows a non linear dependence on the driving field [50].

\subsubsection{Motivations}

The reduced 1D model that treats the domain wall as a particle is of limited applicability to study DW motion in extended thin films with disorder. In fact, in two dimensional systems domain walls no longer behave as particles but more like elastic strings, where magnetization tends to be non-uniform along the domain wall length.
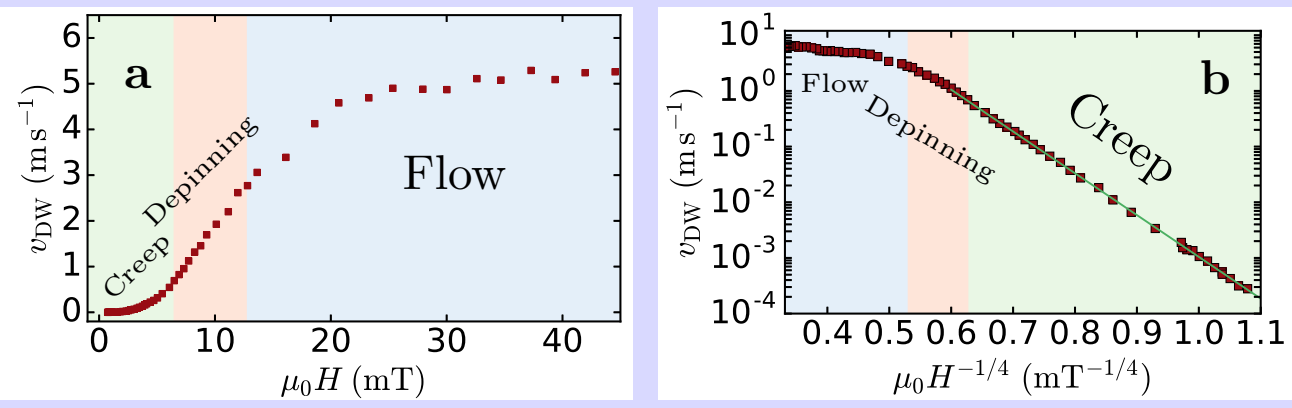

FIGURE 3.1: Different visualization of experimental data of field driven bubble expansion in thin PMA films. a. Standard fieldvelocity diagram where the three different regimes are mareked with different shadings. b. Same data presented in logarithmic scale versus $H^{-1 / 4}$ to show the exponential law relationship in the creep regime. Data are courtesy of L. Herrera Diez (Institut d'Électronique Fondamentale, Université Paris-Sud).

Due to disorder, it exists a depinning field $\left(\mathbf{H}_{\mathrm{dep}}\right)$ below which confinement forces are stronger than driving forces and no domain wall motion is expected. At finite temperature, the domain wall can move for fields lower than $\mathbf{H}_{\text {dep }}$ via thermally activated localized depinning events, i.e. jumping from a local minimum to the next. This dynamics has been investigated in thin films with high PMA [11, 51, 9, 52] and it has been found that DW velocity dependence on applied field follows a very general law that describes the motion of an elastic interface in a medium with weak disorder $[11,53,54,55]$, the so-called creep regime. Creep theory describes well domain wall dynamics at very low fields and information on the material disorder can be extracted by the fitting of experimental data with this model 3.1-b. It ceases to be effective when approaching the depinning field (see fig. 3.1-b), so that domain wall dynamics in a regime useful for applications cannot be studied with this model. On the other hand, for fields higher than $\mathbf{H}_{\text {dep }}$ the wall accelerates up to a viscous flow regime (fig. 3.1-a) where the velocity shows a wide plateau $[9,56]$. For such high applied fields, the pinning strength is thought to be negligible compared to the Zeeman force that drives DW motion. Therefore, disorder has not been considered to play a significant role in this regime [56] and even claimed to be irrelevant [51]. However, 
an analytical model capable of reproducing extended domain wall dynamics in the flow regime is lacking.

The saturation of DW velocity in the flow regime has been attributed to dissipation effects characterized by the intrinsic damping coefficient $\alpha$ and turbulent precessing dynamics of the DW rather than disorder [56, 9]. Nevertheless, Gilbert damping constant is a phenomenological parameter that allows us to fit experimental data up to a certain accuracy, but it does not provide any insight on how the system actually dissipates energy.

Different contributions to damping during DW propagation are emphasized in [57], where it is shown that the experimental value of damping extracted from FMR measurements is considerably smaller than the one extracted from DW mobility in permalloy strips and, therefore, additional effects arising from DW dynamics need to be considered.

Finally, in systems showing high Dzyaloshinskii-Moriya interaction (DMI), the plateau in the velocity has been explained by blocking [58] and annihilation [59] of vertical Bloch lines (VBL) induced by DMI. However, a similar saturation of DW velocity above Walker Breakdown is observed in systems without or with negligible DMI like the ones considered in our study $[9,52]$.

Micromagnetic simulation appears to be an adequate tool to understand the plateauing of DW velocity, since it allows for a detailed reproduction of the system and the introduction of disorder in a realistic way, making it possible to investigate domain wall motion in these materials in its full complexity. In fact, it was investigated via micromagnetic simulations that material defects and disorder affect the motion of vortex domain walls in soft in-plane magnetized nanostrips [60,61] and it has been proposed that disorder introduces a new mean of dissipating the energy in the system, resulting in an extrinsic contribution to the damping.

The question at the origin of this study is that of uncovering the internal DW dynamics that yields a flow regime velocity different from the one predicted by the one dimensional model, using micromagnetic simulations combined with a realistic model to describe material disorder and with realistic material parameters.

\subsubsection{The chapter's structure}

In this chapter, we combine the considerations on disorder-induced energy dissipation with the study of PMA films. By means of realistic full micromagnetic simulations some light is shed on the role played by disorder and by the material structure of the system, contributing to give a better understanding of the DW dynamics in thin films in the flow regime. Our explanation of the DW dynamics in the plateau velocity regime relies on the crystalline material structure and the disorder introduced in it.

In section 3.2 the technique used to simulate material disorder is introduced, the details of the numerical methods used are presented and it is shown how experimental data can be reproduced quite accurately. 
In section 3.3, we investigate how domain wall dynamics is affected by the variation of one of the parameters that define disorder, which will give us more insight on the role played by disorder in the flow regime. We focus on the different way in which a system with disorder dissipates energy and identify critical events at which peaks in the dissipated power appear. We correlate domain wall velocity with the frequency of such events, which also depend on disorder parameters.

In section 3.4 we move to a higher field regime, where the domain wall dynamics is expected to obey to the one dimensional model prediction. We vary both disorder and damping parameter $\alpha$, finding that the variation of disorder introduces a shift in domain wall velocity that cannot be described as a variation of $\alpha$ even where the domain wall dynamic is well described by the one dimensional model. We identify which feature of disorder is responsible for the velocity shift and describe the mechanism through which the system dissipates extra energy. Finally, following the one dimensional model, we consider the additional energy dissipated by including another dissipative term in the one dimensional energy conservation equation (1.43), allowing us to take into account energy dissipated in the domains.

\subsection{Modelling disorder in CoFeB thin films}

\subsubsection{Depinning field}

In section 1.4 field driven domain wall dynamics has been presented as the consequence of energy minimization in a multi-domain sample. From an initial magnetization configuration, the domain wall moves as the consequence of the expansion of a domain whose orientation is energetically favourable for the system under study, so that a domain expansion implies, in the case of the application of an external field, the reduction of Zeeman energy. The force that moves the domain wall is the opposite of the gradient of such global potential energy (1.10).

Material imperfections in a magnetic sample, such as edge roughness or intrinsic defects like impurities or dislocations in the crystal lattice, can alter the energy density distribution, introducing non-uniformities, local maxima and minima in the energy landscape as shown in fig. 3.2. This non-uniform energy landscape, has an overall negative slope in the direction of domain wall propagation when an external field is applied. However, it can have positive gradient at some places, which means that local forces act on the domain wall opposing its propagation. In that case, it is required a minimum critical force $f_{c}$ for the domain wall to have non-zero velocity. In case of field-driven dynamics a depinning field $\mathbf{H}_{\text {dep }}$ is defined as the minimum external field necessary to overcome the local energy barrier opposing domain wall motion [12]. In fig 3.3 the ideal domain wall dynamics is described by the red line, representing the linear regime below Walker Breakdown or the linear regime well above it (see fig.1.11). The blue line describes DW dynamics in presence of material 


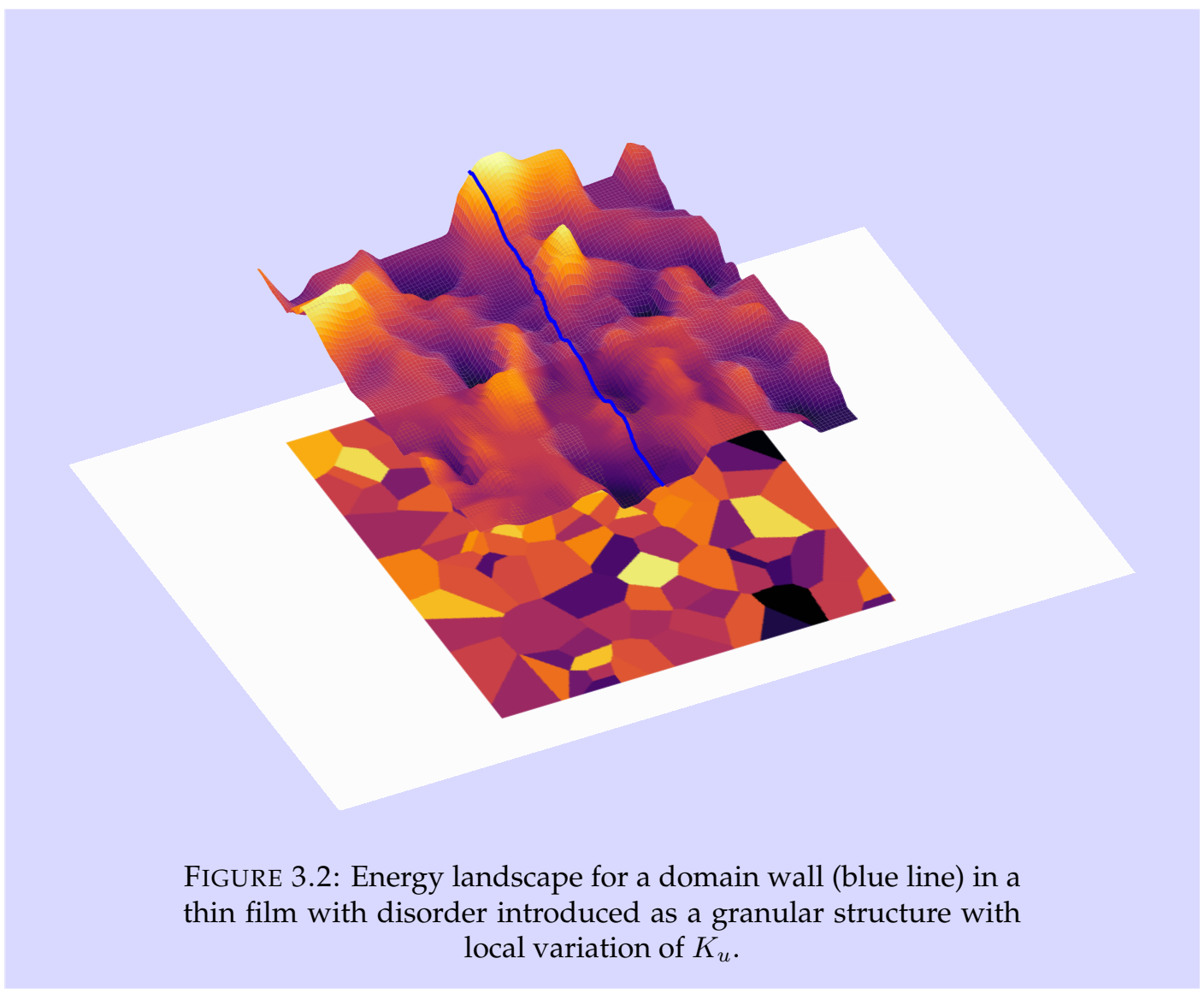

disorder and pinning sites. Due to disorder, there is no motion below the depinning field.

The presence of thermal agitation alters this binary situation in which the domain wall is either at rest or moving. At low fields below $\mathbf{H}_{\text {dep }}$ a domain wall in a strip hops between local minima via thermal activation, behaving as a particle moving with a diffusive Brownian motion and where the applied field only adds a drifting term. In an extended film this picture is complicated by the extension of the DW that is no longer a particle but rather a string showing an internal elasticity: since the domain wall has a linear energy density (1.30), its extension has an energetic cost. Creep theory, developed originally for vortices dynamics in superconductors [62, 55], applies well to domain wall dynamics in PMA thin films [11, 63, 9]. Moreover, temperature smooths out the transition around the depinning point so that experimentally there exists a smooth transition between the domain wall rest and the flow regime. There, different non-linear dynamic regimes appear [64], depending on the competition between thermally activated stochastic motion and deterministic field driven motion, as shown by the dashed green line in fig. 3.3, making more difficult an experimental detection of the depinning field [64, 12]. 


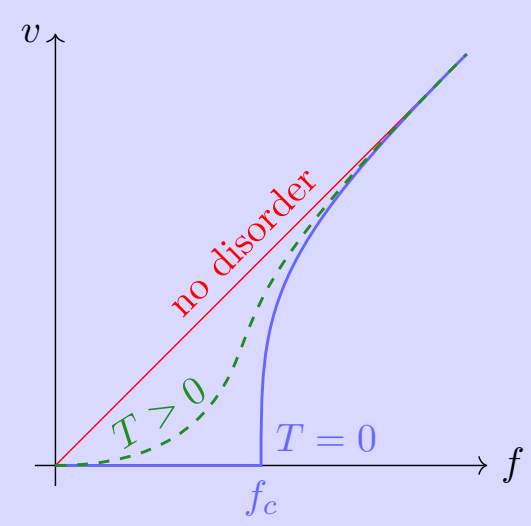

FIGURE 3.3: Domain wall velocity versus driving force in three different systems: ideal system with no disorder (red line) realistic system with disorder at zero temperature (blue line), disordered system where the action of thermal activation is taken into account (green dashed line).

\subsubsection{Disorder in simulations}

Reproducing the effects of disorder in micromagnetic simulations is not a simple task. Most of the features of disorder take place at the atomic scale (vacancies, dislocations, impurities) so that their effect cannot be well reproduced with the nanometer-sized micromagnetic cell discretization. Various techniques for introducing disorder in micromagnetic simulations exist: the inclusion of non-magnetic cells in the magnetic sample $[65,66]$, the variation of thickness by removing micromagnetic cells or reducing saturation magnetization locally [67], the reduction of stiffness constant $A$ at grains boundaries in a regular granular structure [68], the variation of saturation magnetization over a realistic granular structure [60, 61].

All the studies mentioned above, however, refer to domain wall dynamics in in-plane magnetized materials, Permalloy in most of the cases. Since this study concerns DW motion in $\mathrm{CoFeB}$ thin films sandwiched between tantalum and $\mathrm{MgO}$, it is useful to focus on the features of this material. CoFeB grows amorphous with a rather homogeneous texture upon sputtering. To enhance the uniaxial out-of-plane anisotropy in such stacks, an annealing post-deposition procedure is common [9, 69]. Upon annealing, CoFeB thin films crystallize [70, 71, 72], creating a polycrystalline structure with an average grain size between 8 and $20 \mathrm{~nm}$.

As described in the previous chapter (2.2.3), the granular structure of a material can be well described in the micromagnetic code by a Voronoi tessellation of plane. In particular, different grains will show different thickness and a different interface with the overlying $\mathrm{MgO}$ layer. This will mean a different contribution to the surface anisotropy constant. Since in the micromagnetic code the value of $K_{s}$ is included in $K_{u}$, we will focus on the spatial variation of this parameter.

Disorder is introduced in our model by generating a grain distribution with average grain diameter $d$. Typical grain distributions are shown in figure 3.2 and 3.4. 
Both uniaxial anisotropy constant $K_{u}$ and easy axis $\hat{u}$ orientation values are assigned to each grain randomly with a normal distribution around their nominal value with a standard deviation $\sigma$ (see fig. 3.4):

$$
\begin{aligned}
& K_{u, i}=\mathcal{N}\left(K_{u}, \sigma\right)_{i} \quad \hat{u}_{i}=\mathcal{N}(\hat{u}, \sigma)_{i}, \\
& i=1 \ldots N_{\text {grains }}
\end{aligned}
$$

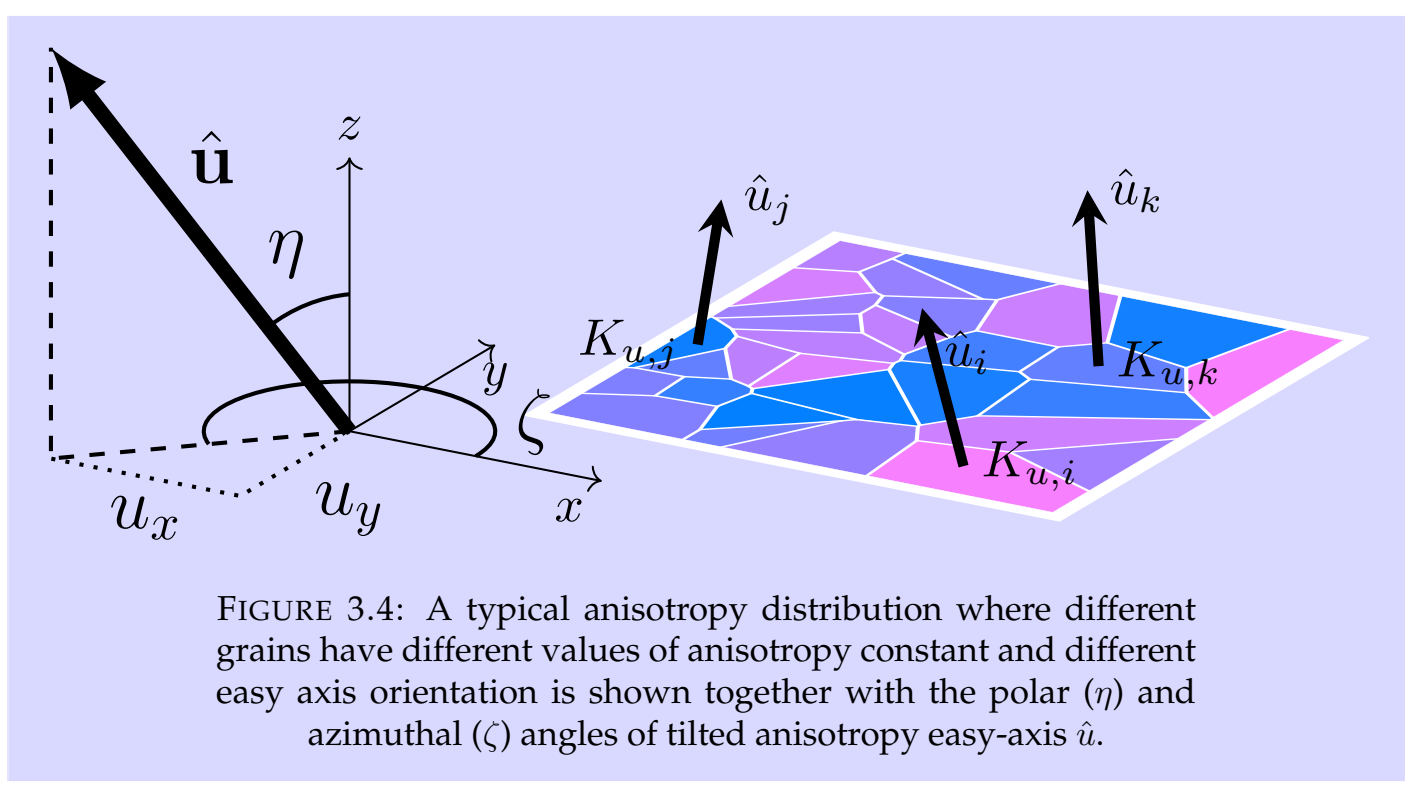

The perturbation of the easy axis orientation in a given grain of our sample can be expressed in the following way:

$$
\begin{aligned}
& u_{x}=n_{x} \\
& u_{y}=n_{y} \\
& u_{z}=\sqrt{1-n_{x}^{2}-n_{y}^{2}}
\end{aligned}
$$

where $n_{x}, n_{y} \in \mathcal{N}(0, \sigma)$ are normally distributed random numbers of zero mean value and standard deviation $\sigma$. The distribution of in-plane orientation among grains can be observed in figure 3.5-a for a case with $\sigma=0.05$. The averaged easyaxis vector is pointing out of the plane with no in-plane component.

If, instead of plotting the distribution of the in-plane components $\left(u_{x}, u_{y}\right)$, we plot the distribution of the spherical coordinates $(\eta, \zeta)$ defined as:

$$
\begin{aligned}
\eta & =\arccos \left(u_{z}\right) \\
& =\arcsin \left(\sqrt{u_{x}^{2}+u_{y}^{2}}\right) \\
& =\arcsin \left(\sqrt{n_{x}^{2}+n_{y}^{2}}\right) \\
\zeta & =\arctan \left(\frac{n_{y}}{n_{x}}\right),
\end{aligned}
$$


we get the plots shown in figure 3.5-b. One can see in fig. 3.5-a (and inset of fig. 3.5b confirms it) that the angle $\zeta$ that describes the orientation of $\hat{u}$ in the xy-plane is uniformly distributed on $[0,2 \pi)$. The $\eta$ angle, on the other hand, describes the tilt from the $z$ axis and its values are always positive (3.1). The distribution that best represents our angle $\eta$ is the Rayleigh distribution [73], defined as the in plane displacement of a particle perturbed with two Gaussian distributions along the coordinate axes. Indeed, for small perturbations, $\arcsin (x) \simeq x$ and so $\eta \simeq \sqrt{n_{x}^{2}+n_{y}{ }^{2}}$. This means that looking at a randomly chosen easy-axis in our sample, one expects to find it tilted away from the z-axis of $\sigma \sqrt{\pi / 2}$ radians, which is the mean value for a Rayleigh distribution originating from Gaussian distributions with standard deviation $\sigma$. The Rayleigh distribution fit is shown with a red line in fig. 3.5-b.
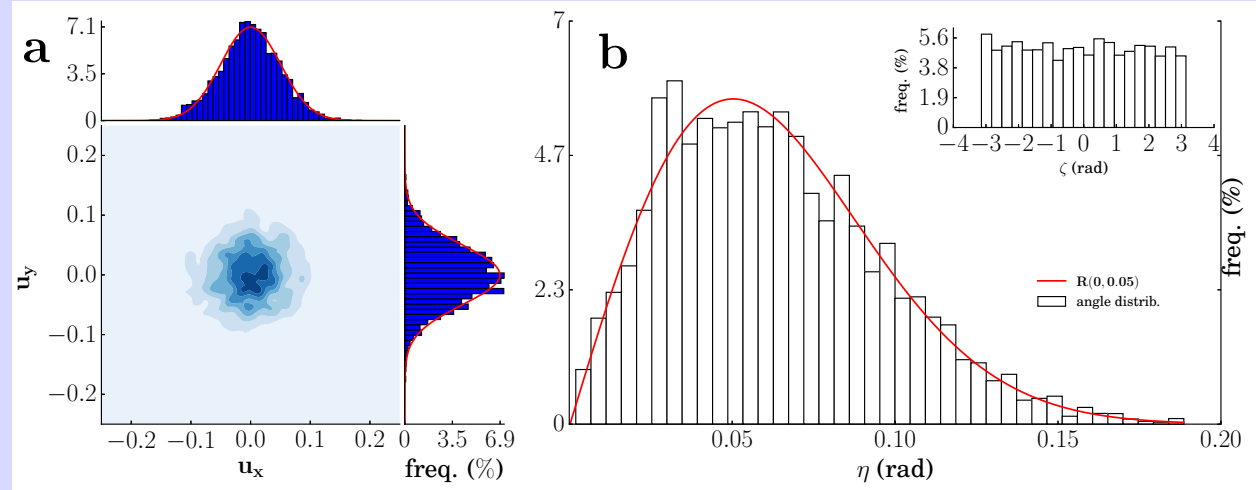

FIGURE 3.5: a. Distribution of the in-plane components of $\hat{u}$ for a sample with grain size $d=30 \mathrm{~nm}$. A Gaussian distribution of standard deviation $\sigma=0.05$ and mean 0 is used to perturb the $x$ and $y$ components at each grain. $\mathbf{b}$. Corresponding distribution of the polar $(\eta)$ and azimuthal $(\zeta$, inset) angles of the easy-axis for the same sample. Red line is the Rayleigh distribution.

\subsubsection{Numerical fit of experimental data}

The system under study is schematically represented in figure 3.6. A domain wall (gray, not on scale) separating up (red) and down (blue) domains is considered. An external field $\mathbf{H}_{\text {ext }}$ is applied perpendicular to the plane favouring the motion of the wall towards the right. Our system is intended to mimic the annealed $\mathrm{Ta} / \mathrm{Co}_{20} \mathrm{Fe}_{60} \mathrm{~B}_{20} / \mathrm{MgO}$ thin films studied experimentally in [52], and, consequently, we have used the material parameter values reported there and also in [9], where similar thin films were considered: exchange constant $A=23 \times 10^{-12} \mathrm{~J} \mathrm{~m}^{-1}$, anisotropy constant $K_{u}=8.34 \times 10^{5} \mathrm{~J} \mathrm{~m}^{-3}$, saturation magnetization $M_{s}=8.83 \times 10^{5} \mathrm{~A} \mathrm{~m}^{-1}$ and Gilbert damping constant $\alpha=0.015$. The computational region is $2 \times 2 \mu \mathrm{m}^{2}$ and the film thickness is $1 \mathrm{~nm}$. The DW structure in these systems, studied elsewhere $[74,75]$, does not present relevant deviations from Bloch structure, and we do not consider the effect of DMI in our simulations. To study the DW dynamics 


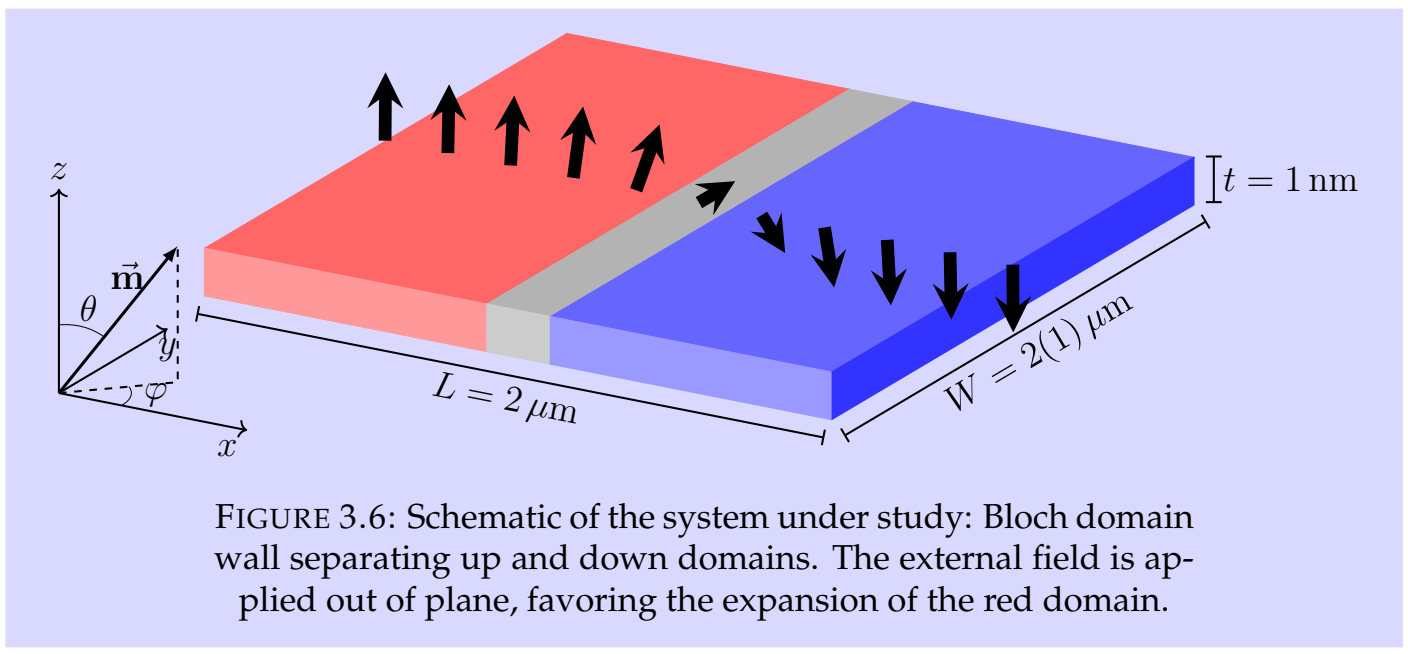

we integrate the Landau-Lifshitz-Gilbert equation (1.6) numerically discretizing the computational region in $4 \times 4 \times 1 \mathrm{~nm}^{3}$ cells.

We introduce thermal fluctuations in our model that correspond to a laboratory temperature $T=300 \mathrm{~K}$. The RK4 scheme is used to numerically integrate the stochastic LLG equation with a fixed time step $\Delta t=4 \times 10^{-13} \mathrm{~s}$. The DW velocity is measured from the change in the average normalized magnetization along the z-axis, $\Delta\left\langle m_{z}\right\rangle$, as

$$
v=\frac{1}{2} L_{x} \Delta\left\langle m_{z}\right\rangle \Delta t_{w}{ }^{-1},
$$

where $L_{x}=2 \mu \mathrm{m}$ is the length of the computational region and $\Delta t_{w}$ is the duration of the simulations. Time windows $\Delta t_{w}=100 \mathrm{~ns}$ and $200 \mathrm{~ns}$ have been considered for the results presented in this section. For high fields, however, the time window is not fixed but the simulation is stopped once $\left\langle m_{z}\right\rangle$ reaches a certain threshold value, typically $\left\langle m_{z}\right\rangle=0.8$. In any case, the velocity is always calculated using (3.2) and the value obtained does not depend noticeably on the time window considered.

In order to fit experimental data, for each grain size $d$ we generate three samples with the same material parameters but different grain distributions. For every value of the external field, three different stochastic realizations are performed for each sample for a total of nine realizations for each point.

Figure 3.7 shows the DW velocity as a function of the external field obtained from micromagnetic simulations considering a perfect film (blue diamonds) and a film with a grain distribution of average size $d=10 \mathrm{~nm}$ and dispersion $\sigma=5 \%$ (black pentagons). These data are compared with the DW velocities measured experimentally in the $\mathrm{Ta} / \mathrm{CoFeB} / \mathrm{MgO}$ thin films from where we took our material parameter values (red circles). The data correspond to the non-irradiated film in figure 1(a) and 1 (b) of [52]. The values of $d=10 \mathrm{~nm}$ and $\sigma=5 \%$ for the grain distribution in the sample with disorder have been chosen in order to obtain a depinning field $\mathbf{H}_{\text {dep }}$ at $0 \mathrm{~K}$ equivalent to the propagation field $\mathbf{H}_{\mathrm{p}}=8.9 \mathrm{mT}$ measured experimentally as the value of $\mathbf{H}_{\text {ext }}$ for which the DW leaves the creep regime. 


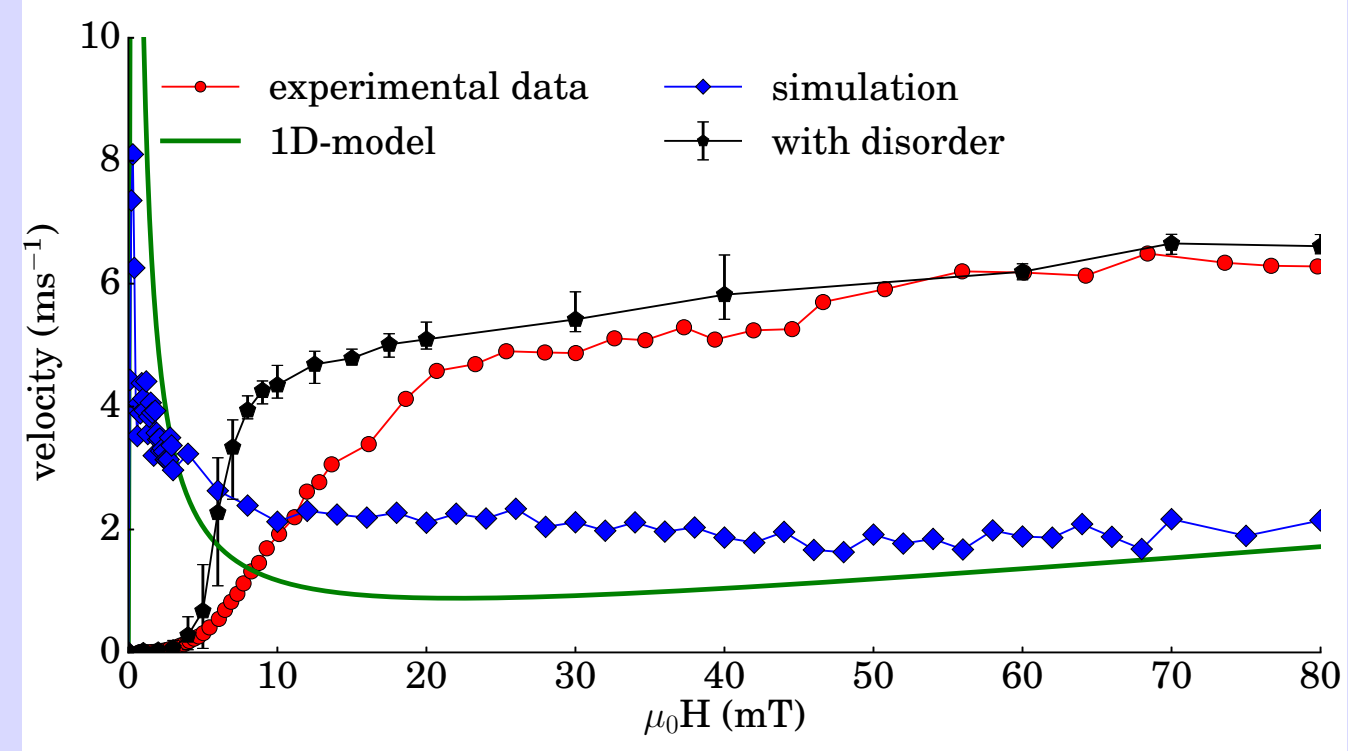

FIGURE 3.7: DW velocity as a function of the applied field. Experimental data courtesy of L. Herrera Diez, (Institut d'Électronique Fondamentale, Université Paris-Sud).

For the perfect film, a rapid linear increase of the DW velocity is obtained for fields below the Walker breakdown $[76,56,63] H_{W} \simeq 0.4 \mathrm{mT}$, difficult to appreciate in the figure, above which a drop in the velocity is found, leading to a large plateau region where the DW moves with incoherent precession and a velocity hardly dependent on the external field $[56,63,9,77,78]$. The low-field linear regime is not observed in the experimental data, since the propagation field in the samples, $\mathbf{H}_{\mathrm{p}}=8.9$ $\mathrm{mT}$, is well above Walker breakdown. Instead, typical thermally activated creep regime $[11,53,51]$ is observed. This regime has been characterized in detail for the system under study elsewhere $[9,52]$ and it will not be discussed here. A thermally activated regime is also obtained with simulations for the sample with grains, as observed in fig. 3.8 at low fields, although a sharper transition around the propagation field $\mathbf{H}_{\mathrm{p}}$ is obtained in this case (see fig. 3.7), due to the limitations imposed by the extension of the time window. In fact, an accurate modelling of this regime would require much larger time scales than the ones considered here, which makes it prohibitive from the computational point of view [79].

For larger fields $\left(\mathbf{H}_{\text {ext }}>20 \mathrm{mT}\right)$ the experimental data show a viscous flow regime in which the velocity is weakly dependent on the external field. As can be observed, the velocity values in this regime are well reproduced in the simulations for the sample with grains, whereas significantly lower values are obtained for the perfect film. This indicates that disorder plays an important role in the viscous flow regime up to field values that are much larger than depinning fields. It is also important to note that, as can be observed in fig. 3.8,temperature does not affect 
significantly the domain wall velocity in the flow regime.
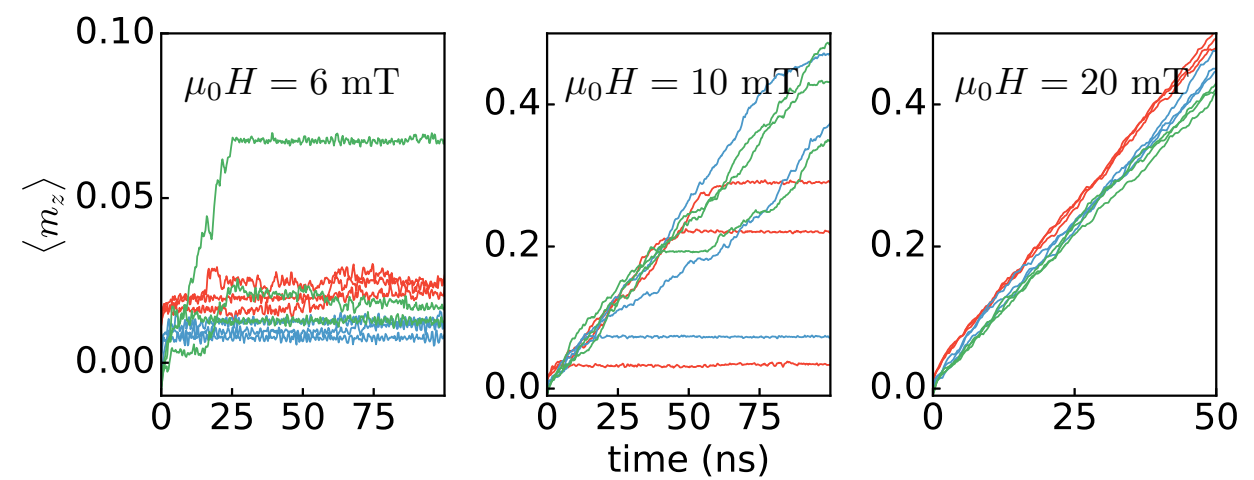

FIGURE 3.8: Average $m_{z}$ component describing DW position as function of time. Three different realizations of disorder (different colors) are presented; each for three different stochastic realizations with thermal field. Thermal activation dominates domain wall dynamics for fields below the depinning threshold as in the $6 \mathrm{mT}$ case. It can be observed to play an important role in the depinning regime $(10 \mathrm{mT})$, while becomes negligible in the flow regime $(20 \mathrm{mT})$.

\subsection{Domain wall velocity and energy dissipation}

\subsubsection{Energy dissipation in ideal and disordered samples}

To understand how disorder affects domain wall motion, in fig. 3.9-a we compare snapshots of the magnetization distribution in the perfect sample (i, ii, iii) with those in a sample with grains (iv, v, vi) at the same consecutive instants of time for the same value of the applied field $\mathbf{H}_{\text {ext }}=40 \mathrm{mT}$ in simulations without thermal noise. In the sample without grains DW displacement takes place via the precession of the spins inside the wall. In an infinite film the wall would be straight and all the spins inside it would precess coherently. Since we are considering a strip of finite width $\left(L_{y}=2 \mu \mathrm{m}\right)$ the effective field is not uniform across the width of the sample due to the surface charges forming at the edges, leading to asynchronous precession of the spins inside the wall and to a slight wall deformation [77]. Nevertheless, taking into account that the color scale in fig. 3.9-a represents the orientation of the inplane component of the magnetization, it is clear that precession takes place counterclockwise across the wall length. In the presence of disorder, however, this global precessing motion pattern is partially lost and overrun by a more chaotic dynamics, which leads to a more pronounced deformation in the DW as observed in snapshots iv, $v$ and vi of fig. 3.9-a. 

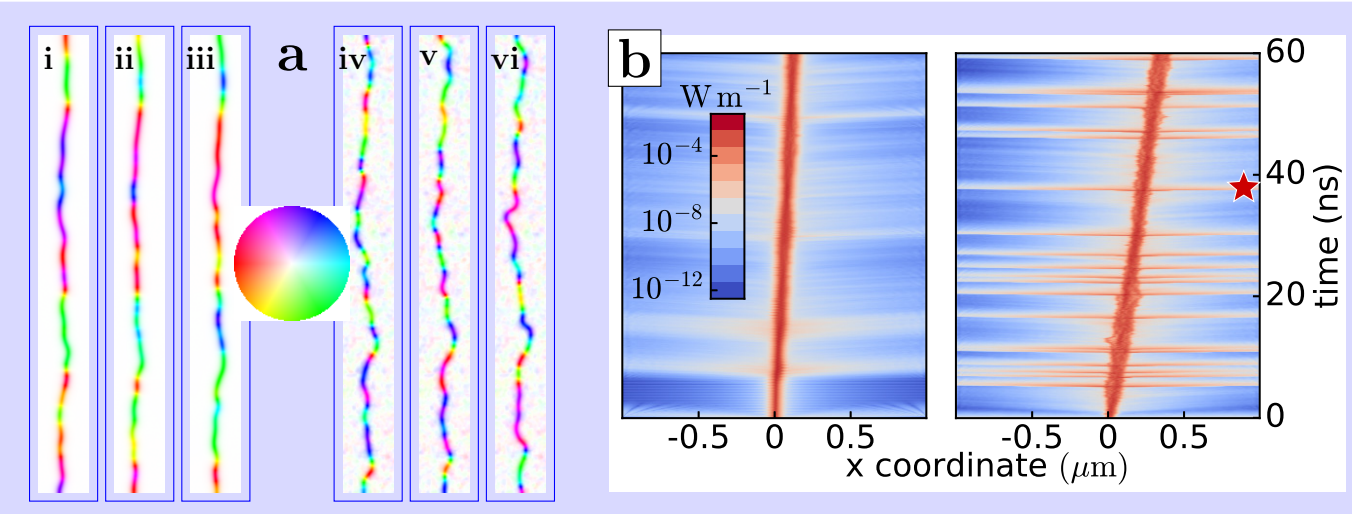

FIGURE 3.9: a. Snapshots of magnetization configuration under an applied field $\mathbf{H}_{\text {ext }}=40 \mathrm{mT}$ without thermal field contribution for a sample without disorder (i,ii,iii) and for a disordered sample (iv,v,vi). Magnetization is displayed as in-plane component at $t=40 \mathrm{~ns}$ (i, iv); $t=40.1 \mathrm{~ns}$ (ii, v); $t=40.2 \mathrm{~ns}$ (iii, vi). b. Time evolution of the dissipated power linear density for the same films as in (a). It can be seen how in the perfect film (left) the energy is dissipated almost exclusively by the spins precessing inside the DW, while the presence of grains and disorder causes the emission of spin waves as an additional channel to dissipate energy in the lattice. The red star marks the critical event represented in fig 3.10 .

To have a deeper understanding of the qualitative difference in domain wall dynamics as it appears from fig. 3.9-a, we can look at how the system dissipates energy. We can extract the dissipated energy density at every micromagnetic cell, at every time step, following a formula for energy density dissipation 1.38 derived from LLG equation and save it every $N$ iterations as

$$
\Delta \mathcal{E}\left(\mathbf{r}, T_{k}\right)=\mu_{0} M_{s} \sum_{i=1}^{N} \Delta \mathbf{m}\left(\mathbf{r}, h_{i, k-1}\right) \cdot \mathbf{H}_{\mathrm{eff}}\left(\mathbf{r}, h_{i, k-1}\right)
$$

where $\Delta \mathbf{m}\left(\mathbf{r}, h_{i, k}\right)$ is the magnetization increment at time $h_{i, k}=i h+T_{k}$ provided by the numeric solver and $\mathbf{H}_{\text {eff }}\left(\mathbf{r}, h_{i, k}\right)$ the corresponding effective field. Dissipated energy distribution allows us to observe the areas that are more affected by energy dissipation and any relevant localized event of energy dissipation that takes place during the dynamics. In fig. 3.10 the spatial distribution of dissipated energy density is plotted for subsequent instants of time and a sudden burst of intense energy dissipation, starting at $t=37.3 \mathrm{~ns}$ is shown. These bursts will be better described in the next section. For the moment we term them critical events.

The critical events start at a localized position along the domain wall and are followed by the emission of spin waves in every direction. If we integrate $\Delta \mathcal{E}(\mathbf{r})$ along $y$ and $z$ direction and divide by the time interval, we obtain the linear dissipated power density along the $x$ direction. The time evolution of this linear dissipated 


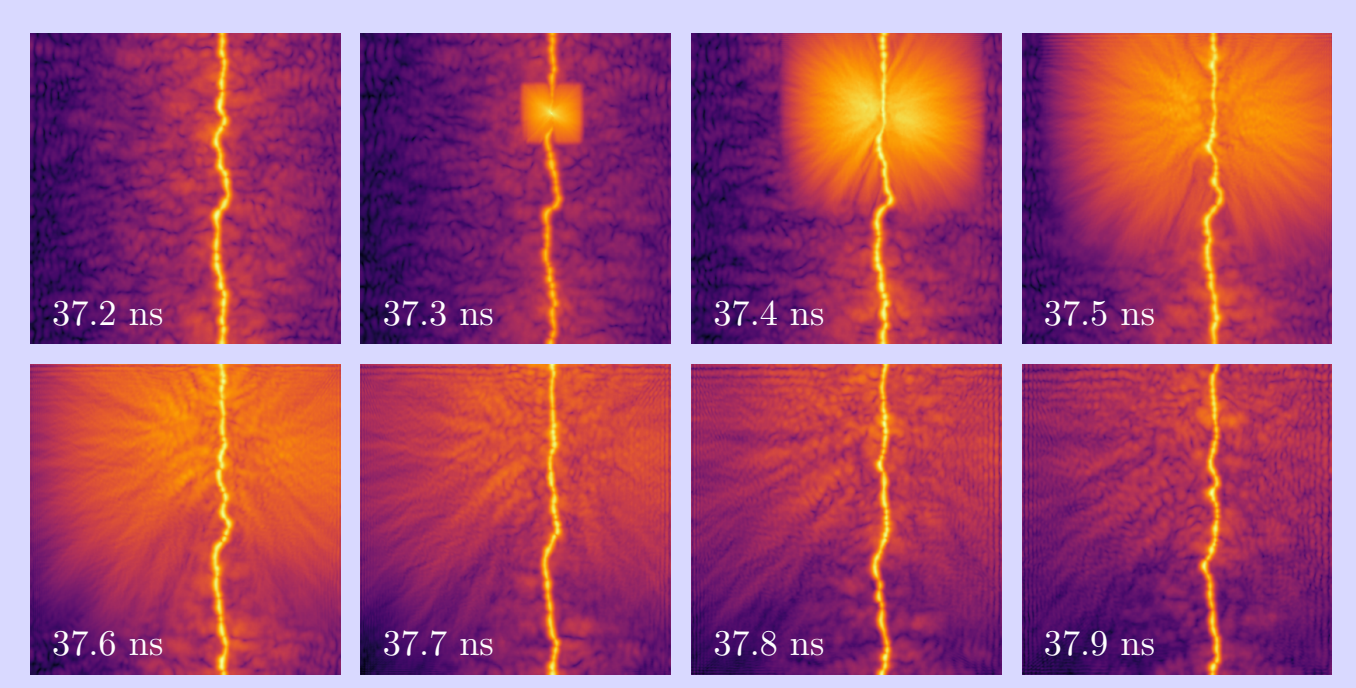

FIGURE 3.10: Successive snapshots representing the instantaneous dissipated energy density distribution. A critical event takes place at $t=37.3 \mathrm{~ns}$ with subsequent emision of spin waves into the two domains.

power density is plotted in fig. 3.9-b. Most of the power is dissipated by the precession of the spins inside the wall, as highlighted by the dark central region that is gradually displaced to the right, due to the fact that the wall is moving, but horizontal fringes are noticeable, corresponding to the energy dissipated through the emission of spin wave packets that originate at the wall and propagate forwards and backwards (the fringe marked with a red star corresponding to the event described in fig. 3.10). Note that the horizontal fringes have only a slight positive vertical component, indicating that the spin waves propagate at much higher velocity than the DW. This effect had already been observed in permalloy strips [60, 61], and interpreted as an extrinsic effective damping: an additional means for the system to dissipate energy through the emission of spin waves at the many DW local critical events, leading to a faster relaxation of the system and to a faster DW motion.

We find that disorder has a large influence on DW motion, not only at low fields but also in the plateau region, in contrast with what has been claimed elsewhere [56, 51]. We can introduce material disorder in micromagnetic simulations in a realistic way, supported by the experimental evidence of grain structure and defects in thin films of annealed $\mathrm{CoFeB}[9,80,81]$, while keeping the intrinsic damping parameter value measured experimentally. In this way, we can obtain DW velocity values in the flow regime close to the ones measured experimentally, and, at the same time, preserve the internal DW dynamics that would be suppressed with the use of a large damping parameter. Moreover, we can tune the description of disorder by changing the disorder characteristics such as grain size and local variation of the parameters. 

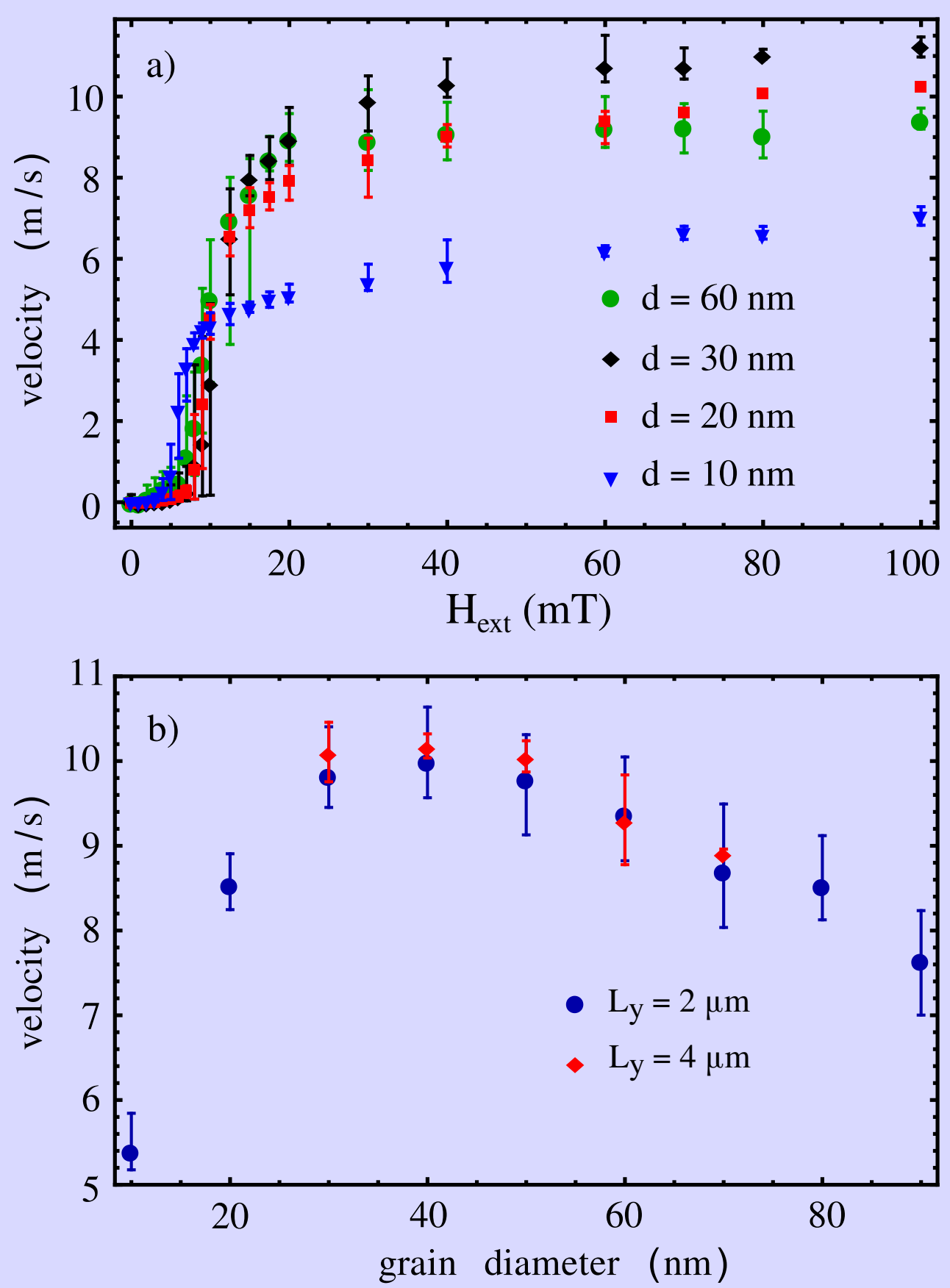

FIGURE 3.11: a. DW velocity as a function of the applied field for various grain size at $T=300 \mathrm{~K}$. b. DW velocity at $\mathbf{H}_{\text {ext }}=40$ $\mathrm{mT}$ and $0 \mathrm{~K}$ as a function of average grain diameter $d$.

\subsubsection{Domain wall velocity and variation of grain diameter}

Once shown that disorder has a strong impact on DW motion in the flow regime, it is useful to know how the different parameters that characterize this disorder affect DW motion. In our model, disorder is determined by a grain structure of size $d$ and dispersion $\sigma$. The effect of dispersion has already been investigated in other numerical works $[60,61,77]$ and it appears clear that an increase in dispersion gives rise 

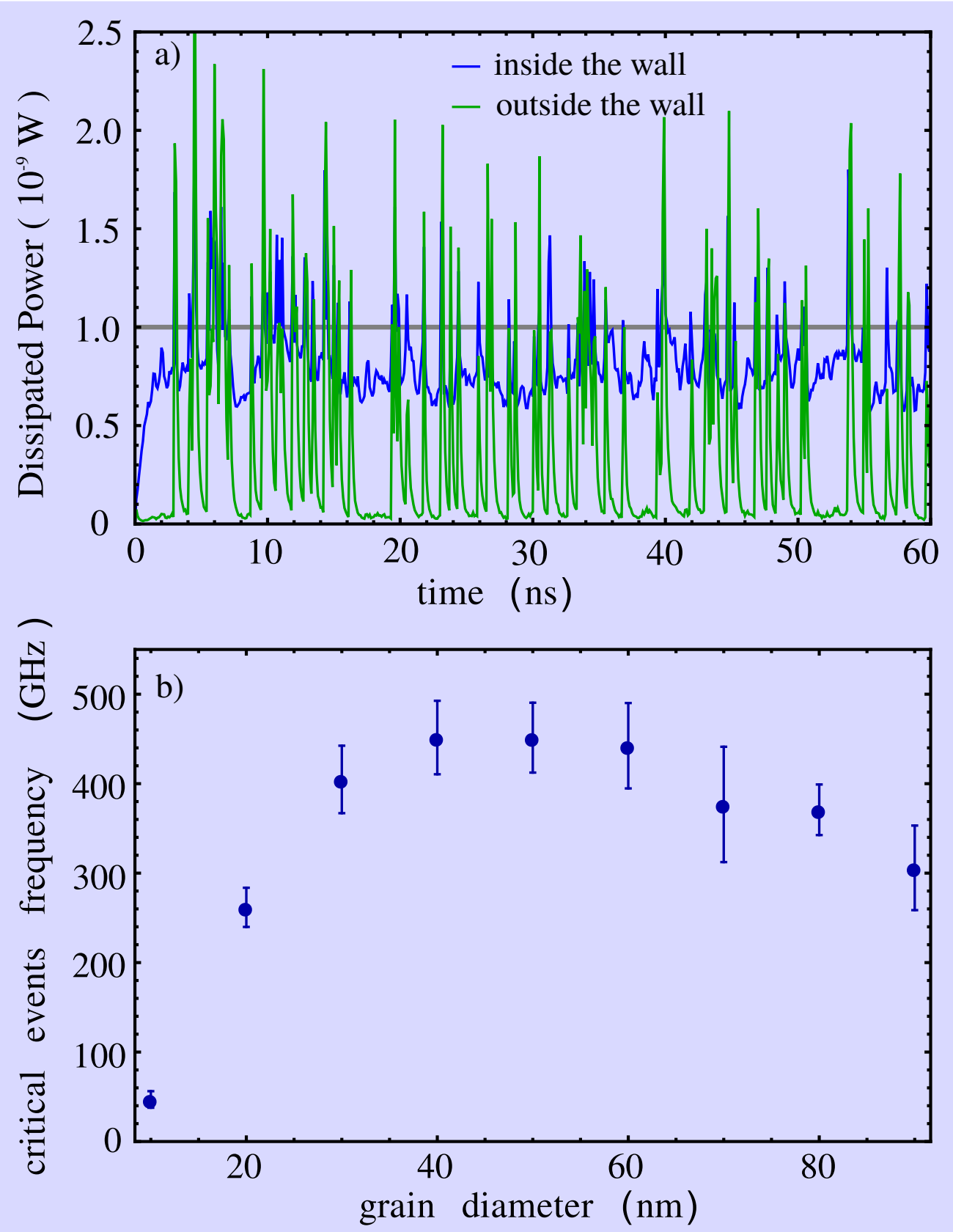

FIGURE 3.12: a. Time evolution of the dissipated power inside and outside the wall for a sample with grain size $d=20 \mathrm{~nm}$. b. Frequency of critical events exceeding a fixed threshold as a function of grain size. Error bars mark standard deviation.

to higher energy barriers between grains, leading to a stronger pinning, therefore to higher propagation fields. In the flow regime, on the other hand, the augmented disorder causes a higher saturation velocity, since it increases the frequency of local critical events and the subsequent dissipation of energy. However, the spatial variation of material parameters with a certain dispersion $\sigma$ is subordinate to a partition of the system in grains. The presence of grains introduces a different dynamic behaviour for the DW and their size is expected to play a role in determining this 
behaviour.

The effect of grain size on DW motion has been investigated before in in-plane magnetized strips in the linear regime below Walker breakdown [61] and it has been found that increasing grain size leads to an increase in extrinsic damping. In our system the competition between the grain size $d$ and the DW characteristic length plays an important role in determining the DW propagation. Moreover, grain size might be controlled experimentally to a certain extent, since in CoFeB the crystalline structure appears upon annealing and is induced by the overlying oxide layer, whose crystalline structure acts as a template for the structure of CoFeB [80, 81].

In what follows we will study how the DW velocity changes with grain size $d$ keeping the same material parameters specified above and $\sigma=5 \%$. The results are presented in figure 3.11-a, where it can be observed that both the propagation field and the DW velocity in the flow regime depend on grain size. In particular, the saturation velocity in the plateau region seems to increase up to a grain size $d=30 \mathrm{~nm}$ and then decrease for $d=60 \mathrm{~nm}$. To further investigate this effect, we compute the DW velocity as a function of the grain size for a fixed external applied field $\mathbf{H}_{\text {ext }}=40 \mathrm{mT}$. In this case the simulations are carried out at $T=0 \mathrm{~K}$, since it is observed that the effect of thermal fluctuations on the DW velocity is negligible. For every grain size ten different samples are generated and simulations are run with stopping criterion $\left\langle m_{z}\right\rangle=0.8$. The results, presented in figure 3.11-b, show that the DW velocity rapidly increases with grain size up to a value $d=40 \mathrm{~nm}$ above which it slowly decreases. Very similar curves (not shown) are observed for other values of $\mathbf{H}_{\text {ext }}$ in the flow regime.

Once we have identified the occurrence of local critical events followed by spin wave emission as the most noticeable mechanism induced by disorder on DW motion in the flow regime, we can try to understand the behaviour shown in figures 3.11-a and 3.11-b in terms of this mechanism. As shown in fig. 3.9-b, spin wave emission can be easily identified by monitoring the energy dissipated in the system. To isolate the spin wave emission events from the other relevant dissipation mechanism, precessional motion of the spins inside the wall, we integrate in space the dissipated power density dividing the computational area into two regions: a band $60 \mathrm{~nm}$ wide centred on the DW and the two remaining domains to the left and right.

Fig. 3.12-a shows the time evolution of the dissipated power in the wall region (blue line) and outside it (green line) for a sample with grains of $20 \mathrm{~nm}$ of diameter under the application of a $40 \mathrm{mT}$ external field. Inside the wall the energy is dissipated at a well defined rate with minor fluctuations, whereas outside the wall the dissipated power consists of a distribution of very pronounced peaks that correspond to critical events. Therefore, by setting a power threshold we can readily count the number of such events and check whether there is any dependence on grain size. The results are shown in figure 3.12-b, where the frequency of events in which the dissipated power exceeds $1 \mathrm{nW}$ is plotted as a function of grain size. As can be observed by comparison with figure 3.11-b, a perfect correlation between 
DW velocity and frequency of critical events is found. It was checked that choosing a different threshold changes the frequency values but their dependence on grain size remains the same. Therefore, it can be concluded that DW velocity in the flow regime is directly related to the frequency of critical events.

After having identified the mechanism behind the DW velocity dependence with grain size, several open questions are left to answer: why the maximum DW velocity is found for a grain size of $40 \mathrm{~nm}$ ? What causes the critical events and how can these be described in terms of magnetization dynamics? How can we account for this additional dynamics introduced by disorder in an analytical model? The next section is dedicated to tanswer to such questions.

\subsection{Influence of disorder at high fields}

As can be observed in fig. 3.7, micromagnetic simulations in an extended film with no disorder are much closer to the 1D model prediction than to experimental data or to micromagnetic simulations with disorder. An important fact that can be noted, when comparing the two numerical results and the analytical model, is that in the higher field regime, above $40 \mathrm{mT}$, the slope of the curves tend to equalize and the micromagnetic simulations without disorder asymptotically tend to the one dimensional prediction.

\subsubsection{Gilbert damping and disorder}

To shed more light on the role played by disorder in increasing domain wall velocity in the flow regime, we move to high applied fields. In this way, we exploit the agreement of standard micromagnetic simulations with the analytical model and decouple the contribution of disorder to DW velocity from the precessional dynamics described by the one dimensional model. We generate different disordered samples, varying grain size between 5 and $30 \mathrm{~nm}$ in diameter and apply external fields up to $360 \mathrm{mT}$. Here, due to the high applied fields, no relevant changes are observed in DW velocity for different realizations of disorder, so that a single disordered thin film is generated for each grain size.

DW velocity measurements from micromagnetic simulations are shown in fig. 3.13a for samples of width $W=2 \mu \mathrm{m}$ with various grain sizes and $\alpha=0.015$, together with the 1D model calculations. As can be observed, for applied fields well above Walker field, the analytical model predicts a non-linear precessional periodic regime of period $T$ for which the following expression for the average velocity 

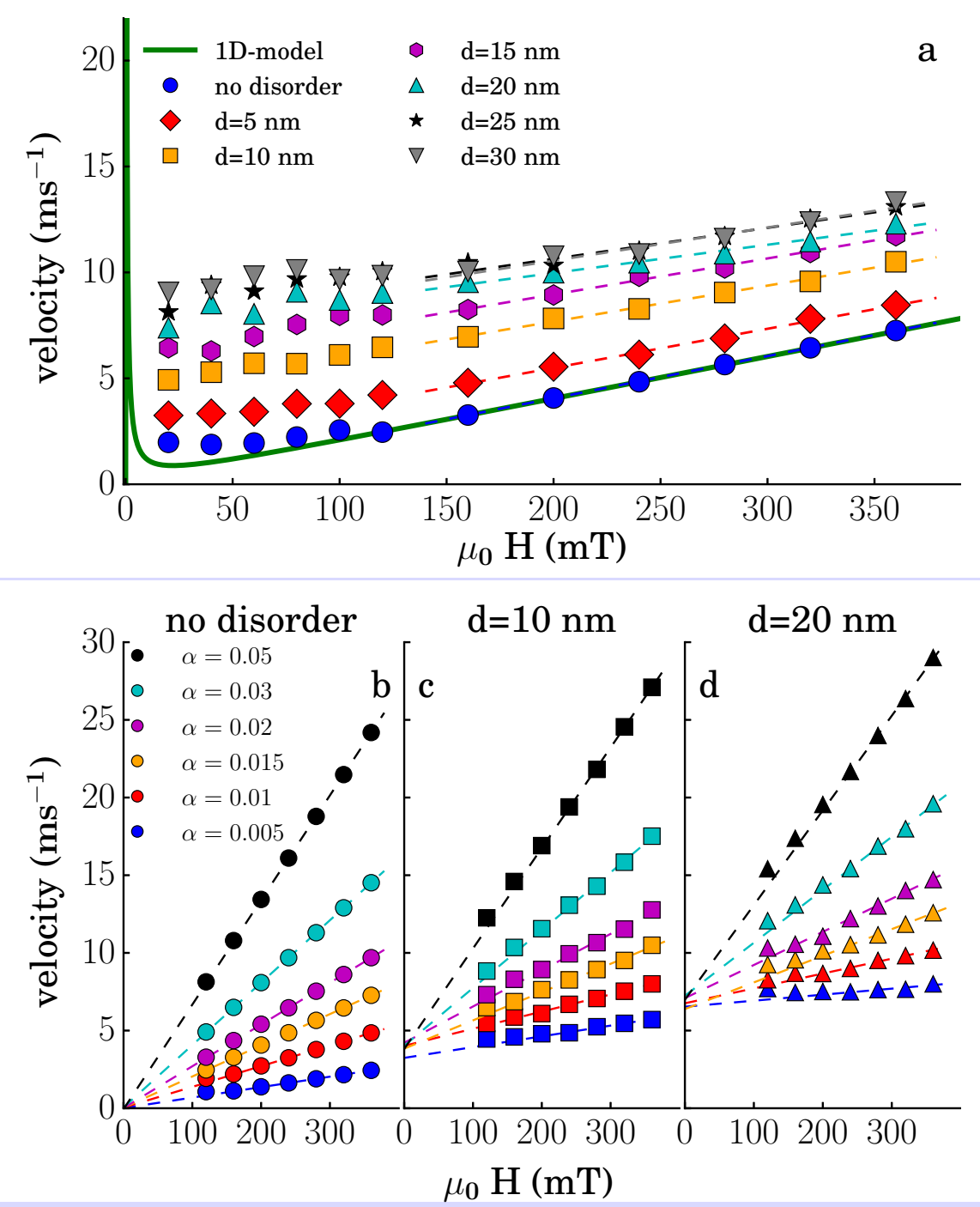

FIGURE 3.13: a. DW velocity as function of applied field from micromagnetic simulations with Gilbert's damping $\alpha=0.015$ and various grain diameters, together with simulations with no disorder and 1D model analytical calculations (green line). b,c,d. DW velocity at high field for various values of $\alpha$ and a fixed grain size, showing the shift in the zero field intercept with introduction of disorder. Dashed lines are linear fittings to the 1D linear precessional regime.

$\langle v\rangle=T^{-1} \int_{T} v \mathrm{~d} t$ (1.76) can be obtained [13]:

$$
\begin{aligned}
v(t) & =\frac{\gamma_{0} \Delta}{1+\alpha^{2}}\left(\alpha H_{a}+H_{K} \frac{\sin 2 \phi}{2}\right) \\
\langle v\rangle & =\frac{\alpha \gamma_{0} \Delta}{1+\alpha^{2}} H_{a}+\frac{\gamma_{0} \Delta}{\alpha\left(1+\alpha^{2}\right)} \underbrace{\left[H_{a}-\sqrt{H_{a}^{2}-H_{W}^{2}}\right]}_{\rightarrow 0 \text { for } H_{a} \gg H_{W}} .
\end{aligned}
$$


On the other hand, a plateau for the velocity is found in simulations of wide wires $[9,50]$. As discussed in the previous section, the existence of this velocity plateau and the deviation from one dimensional model has its origin in the non one dimensional character of the system and the consequent non uniform magnetization along the DW length, generated by non uniformities in the stray field along the DW, which yields a saturation velocity [50]. At higher fields, this stray field inhomogeneity becomes less and less important and a good agreement with the 1D model is found for the sample with no disorder (blue circles), where the spins inside the DW precess coherently. This means that for $H_{a} \gg H_{W}$, the DW in an ideally perfect sample behaves in a quasi-1D fashion, precessing uniformly and showing the linear dependence of velocity on field predicted by eq. (3.4), $\langle v\rangle=\gamma_{0} \Delta H_{a}\left(\alpha+\alpha^{-1}\right)^{-1}$. The introduction of disorder, however, causes a general shift in the velocity both in the plateau and in the precessional linear regime. Velocity is increased by an amount that grows with grain size and saturates at around $25-30 \mathrm{~nm}$ but it is weakly dependent on the applied field. Variation of $\alpha$ parameter affects the dynamics of our system changing the slope of the linear field-velocity relationship, as prescribed by eq. (3.4). Fig. 3.13b-d presents DW velocity measurements for the sample without disorder (b) and samples with grain size $d=10 \mathrm{~nm}$ (c) and $20 \mathrm{~nm}(\mathrm{~d})$, where the velocity values at lower fields have been omitted to make the zero field intercept of the linear fitting clearly visible. It appears that the introduction of disorder gives rise to an additional term of the velocity in the precessional linear regime, changing the velocity expression in this regime to

$$
\langle v\rangle \simeq \frac{\alpha \gamma_{0} \Delta}{1+\alpha^{2}} H_{a}+v_{0}(d)
$$

It is clear that, in the high field linear regime investigated here, a characterization of the effect of disorder as a variation in the damping parameter as done in [57, $61,60]$ does not apply, since disorder does not affect the DW mobility $\mu=v / H$, but introduces a shift in the velocity. Rather, one would tend to think, at least phenomenologically, that the effect of disorder is decoupled from the contribution due to damping.

To get further insight into the effect of disorder on DW dynamics, we consider separately the two different ways in which disorder is introduced in our model, namely the dispersion in $K_{u}$ and $\hat{u}$.

Fig. 3.14-a shows simulation results for a sample with grains of $10 \mathrm{~nm}$ diameter together with the perfect film and simulations of that very sample where the only parameter varying in space is either $K_{u}$ (spades symbol $\mathbf{p}$ ) or $\hat{u}$ (clubs symbol $\mathbf{p}$ ). As can be seen, the influence on DW velocity of the tilting of $\hat{u}$ is striking, while $K_{u}$ spatial variation doesn't seem to play a role. Although variation of both parameters is relevant when studying DW depinning and low field dynamics [57, 61, 77, 82], at high fields the local variation of $K_{u}$ has negligible effects. Our interpretation for this is the fact that the value of $K_{u}$ influences the width and linear energy density of a 


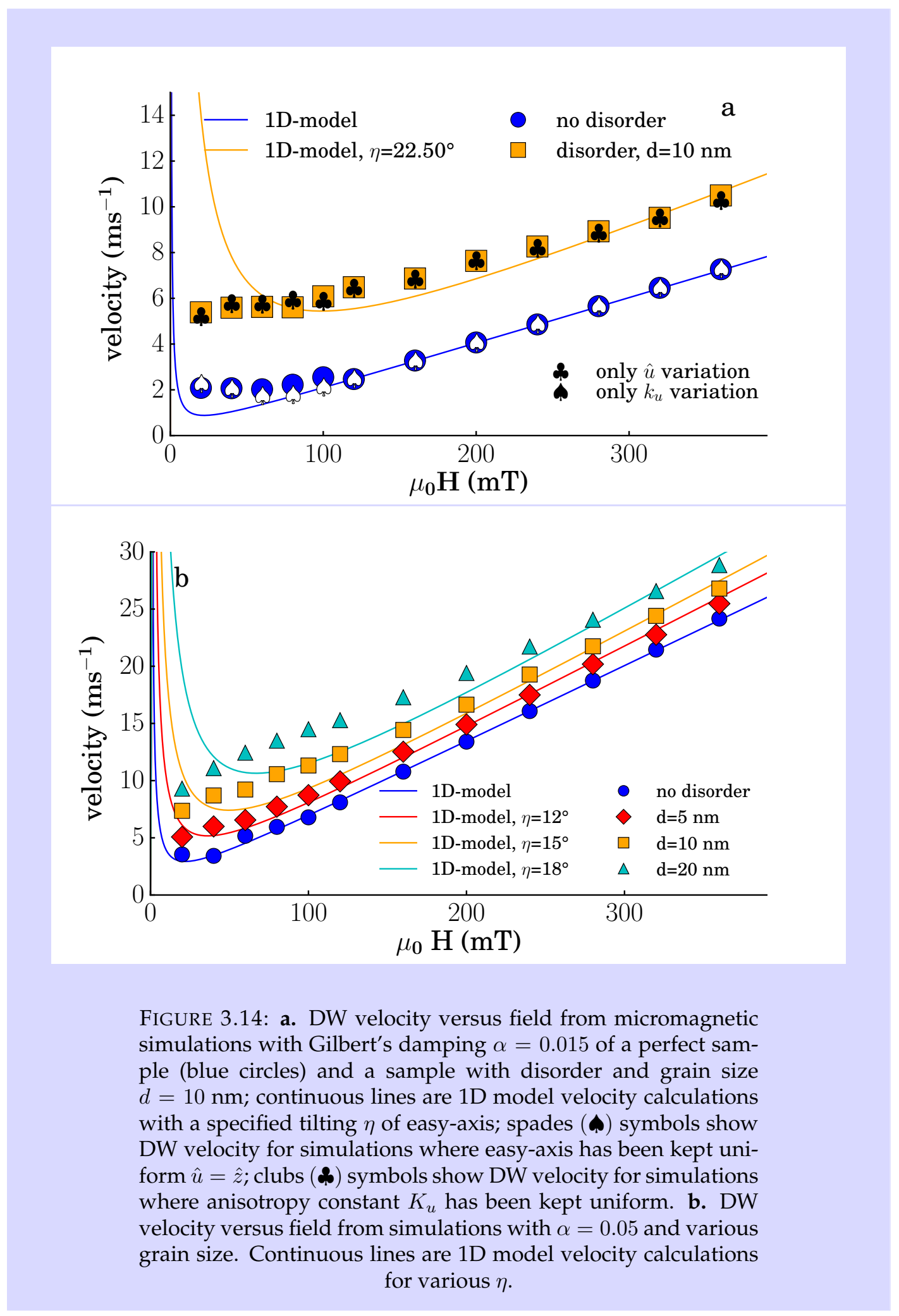

$\mathrm{DW}$, hence playing a bigger role in the statics. A deviation of $\hat{u}$ from the normal to plane, on the other hand, introduces directly an in-plane transverse anisotropy. The effect of in-plane anisotropy on the DW dynamics can be investigated by including it in the 1D model of Slonczewski [50]. The equations of motion for an up-down 
domain wall, eq. (1.65) and (1.66), in case of a tilted anisotropy axis $\hat{u}=\left(u_{x}, u_{y}, u_{z}\right)=$ ( $\sin \eta \cos \zeta, \sin \eta \sin \zeta, \cos \eta$ ) become:

$$
\begin{aligned}
v=\dot{q} & =\frac{\gamma_{0} \Delta}{1+\alpha^{2}}\left(\alpha H_{a}+\frac{H_{K}}{2} \sin 2 \phi-H_{K}^{\prime \prime} \cos 2 \phi\right) \\
& =\frac{\gamma_{0} \Delta H_{W}}{1+\alpha^{2}}\left(\alpha h_{1}+\frac{1}{\alpha}\left(\sin 2 \phi-h_{2} \cos 2 \phi\right)\right) \\
\dot{\phi} & =\frac{\gamma_{0}}{1+\alpha^{2}}\left(H_{a}-\frac{\alpha H_{K}}{2} \sin 2 \phi+\alpha H_{K}^{\prime \prime} \cos 2 \phi\right) \\
& =\frac{\gamma_{0} H_{W}}{1+\alpha^{2}}\left(h_{1}-\sin 2 \phi+h_{2} \cos 2 \phi\right)
\end{aligned}
$$

where $q$ is the 1D model DW position along the strip and

$$
\begin{aligned}
K & =\frac{1}{2} \mu_{0} M_{s}^{2}\left(N_{y}-N_{x}\right)-K_{u}\left(u_{y}^{2}-u_{x}^{2}\right), \\
H_{K}^{\prime \prime} & =\frac{2 K_{u} u_{x} u_{y}}{\mu_{0} M s}, \quad h_{1}=\frac{H_{a}}{H_{W}}, \quad h_{2}=\frac{2 H_{K}^{\prime \prime}}{H_{K}},
\end{aligned}
$$

$N_{x}$ and $N_{y}$ being the DW demagnetizing factors along $x$ and $y$. For fields above Walker breakdown, $h_{1} \geq \sqrt{1+h_{2}^{2}}$, we find again a periodic motion and an average DW velocity

$$
\langle v\rangle=\frac{\gamma_{0} \Delta H_{W}}{1+\alpha^{2}}\left[\alpha h_{1}+\frac{1}{\alpha}\left(h_{1}-\sqrt{h_{1}^{2}-1-h_{2}^{2}}\right)\right]
$$

Eq. (3.10) describes DW velocity in the precessional regime for a DW propagating in one dimensional system with a uniformly tilted anisotropy axis and it is exactly equivalent to (3.4) in the case $\hat{u}=\hat{z}$.

There are several differences between the 1D system described by (3.10) and the system studied in micromagnetic simulations: the easy-axis does not have a uniform direction over the sample, but is randomly tilted from the out of plane direction following a normal distribution of mean $\hat{z}$ and standard deviation $\sigma=5 \%$. This gives a Rayleigh distribution for the polar angle $\eta$ of $\hat{u}$ and a uniform distribution in $[0,2 \pi)$ for its azimuthal angle $\zeta$ as shown in fig.3.5-a and b. A fixed polar and azimuthal angle are required for the 1D model prediction. Since the choice of azimuthal angle $\zeta$ highly affects the dynamics and all orientations have the same probability (see inset of fig.3.5-b), we choose only the polar angle $\eta$ and calculate the velocity as a mean of the values obtained varying $\zeta$ over all the interval $[0,2 \pi)$. 1D model calculations compared to simulations are shown in figure 3.14-a and 3.14-b for values of $\alpha=0.015$ and 0.05 respectively. One readily checks that the tilted polar angle value needs to be much bigger than the actual tilt of $\hat{u}$ in order to reproduce the desired velocity. Moreover, a relationship between grain size and tilt of the easy-axis is lacking. In the next section we focus on these two aspects, bringing in the discussion two features not considered by standard 1D models: internal DW dynamics and energy dissipation outside the wall. 


\subsubsection{Vertical Bloch line dynamics}

A similar saturation velocity in the flow regime as the one of fig. 3.7 and 3.11 has been observed in systems with high DMI $[59,58]$, where spin wave emission takes place upon vertical Bloch lines (VBLs) annihilation in the DW caused by the different propagation velocity of VBLs of same chirality and opposite charge induced by DMI. In that case, the dissipation process has been shown to be independent on the grain structure. Here, on the other hand, we see that for systems without DMI the reason behind the velocity plateau and shift can be found in the interaction of DW with the grain structure.

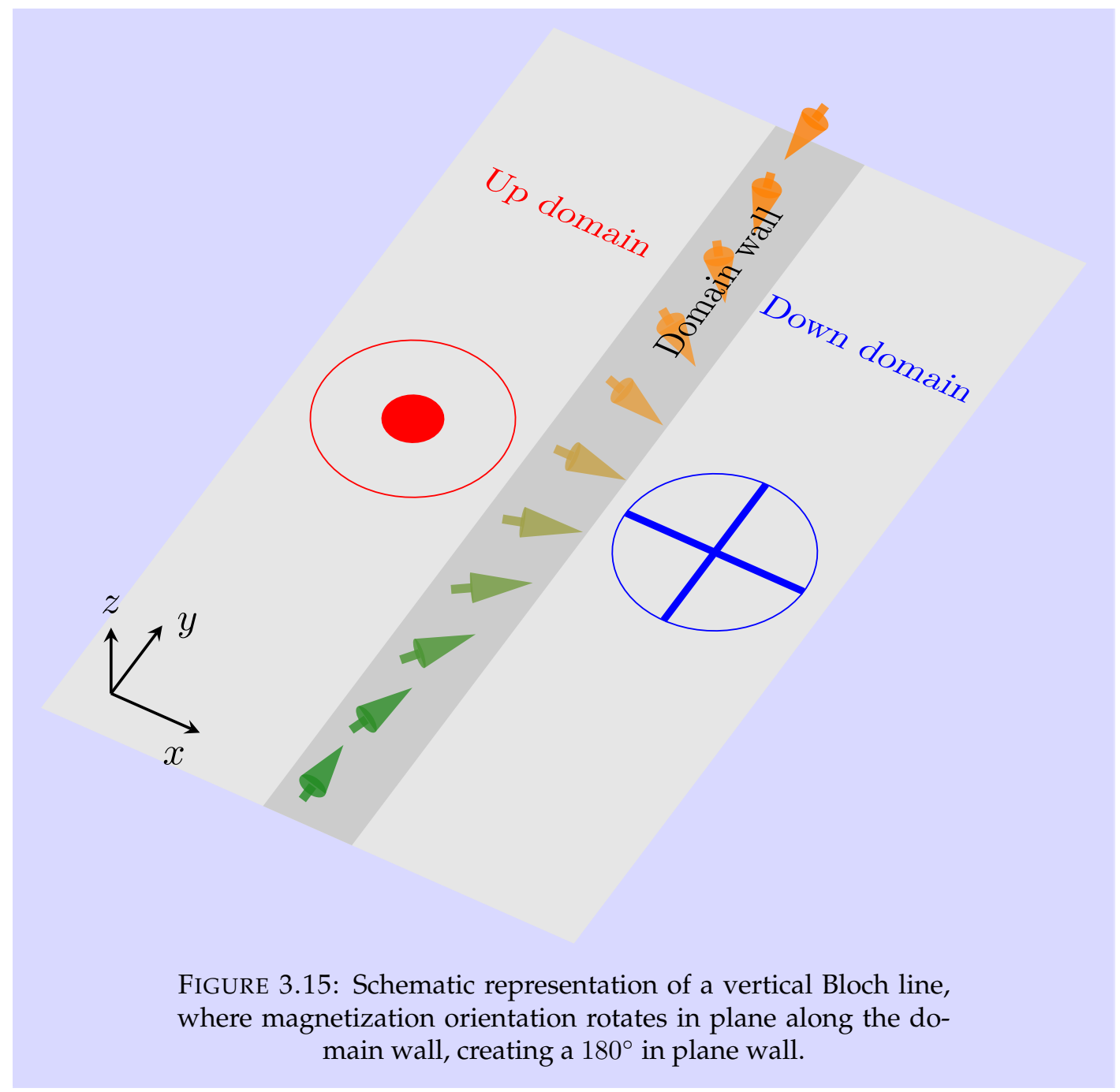

Vertical Bloch lines form when the magnetization rotates along the domain wall length [77], as shown in fig. 3.15. Such magnetic texture is not energetically favourable in a thin film so that VBL will in general not appear at rest. However, in the precessional regime VBL tend to form and propagate along the DW even in absence of disorder (see fig. 3.9-a). They have been studied in the past in thicker films [50], where it was shown analytically that their presence caused a saturation of DW velocity setting a lower bound for domain wall velocity in the precessional regime. 
Vertical Bloch lines, due to their chiral conformation, have a topological number, also named topological charge, defined by the sense of rotation of the magnetization, clockwise or counter-clockwise. This charge $w$ is computed as [83]

$$
w(t)=\frac{1}{4 \pi} \int_{X} \int_{Y} \mathbf{m}(t) \cdot\left(\frac{\partial \mathbf{m}(t)}{\partial x} \times \frac{\partial \mathbf{m}(t)}{\partial y}\right) \mathrm{d} y \mathrm{~d} x .
$$

Moreover, since magnetization deviates from the magnetostatically favourable Bloch state, VBLs have a magnetic charge, positive or negative, whose polarity is defined by the orientation of their core. In an extended domain wall, for an applied field above Walker's field, the presence of defects or surface magnetostatic charges at the edges will produce a non-uniform effective field, DW in-plane rotation becomes then incoherent, since at certain points precession will be easier than at others. Couples of VBLs are nucleated at some position along the domain wall, with same magnetic charge and opposite chirality, and propagate along the DW in opposite direction, as shown in fig. 3.16, pushed by a gyrotropic force that depends on the chirality as [50, $58]$

$$
\mathbf{F}_{\mathrm{VBL}}=\frac{4 \pi M_{s} w}{\gamma} \hat{\mathbf{z}} \times \mathbf{v}_{\mathrm{DW}}
$$

where $\mathbf{v}_{\text {DW }}$ is the local DW velocity. The next couple of VBL will have same magnetic charge, opposite to the ones before, and opposite chiralities, with the VBL moving up having same chirality of its predecessor.

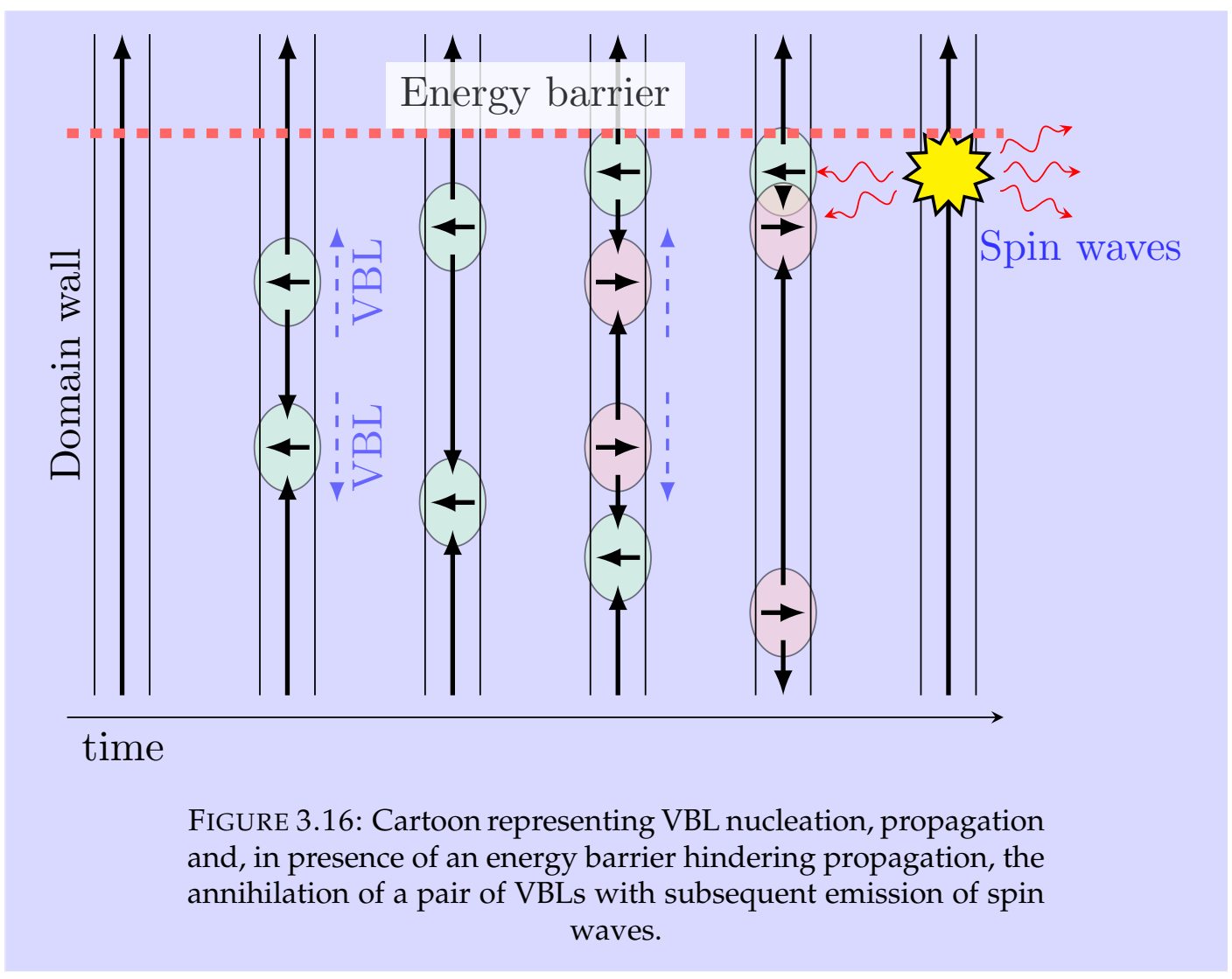




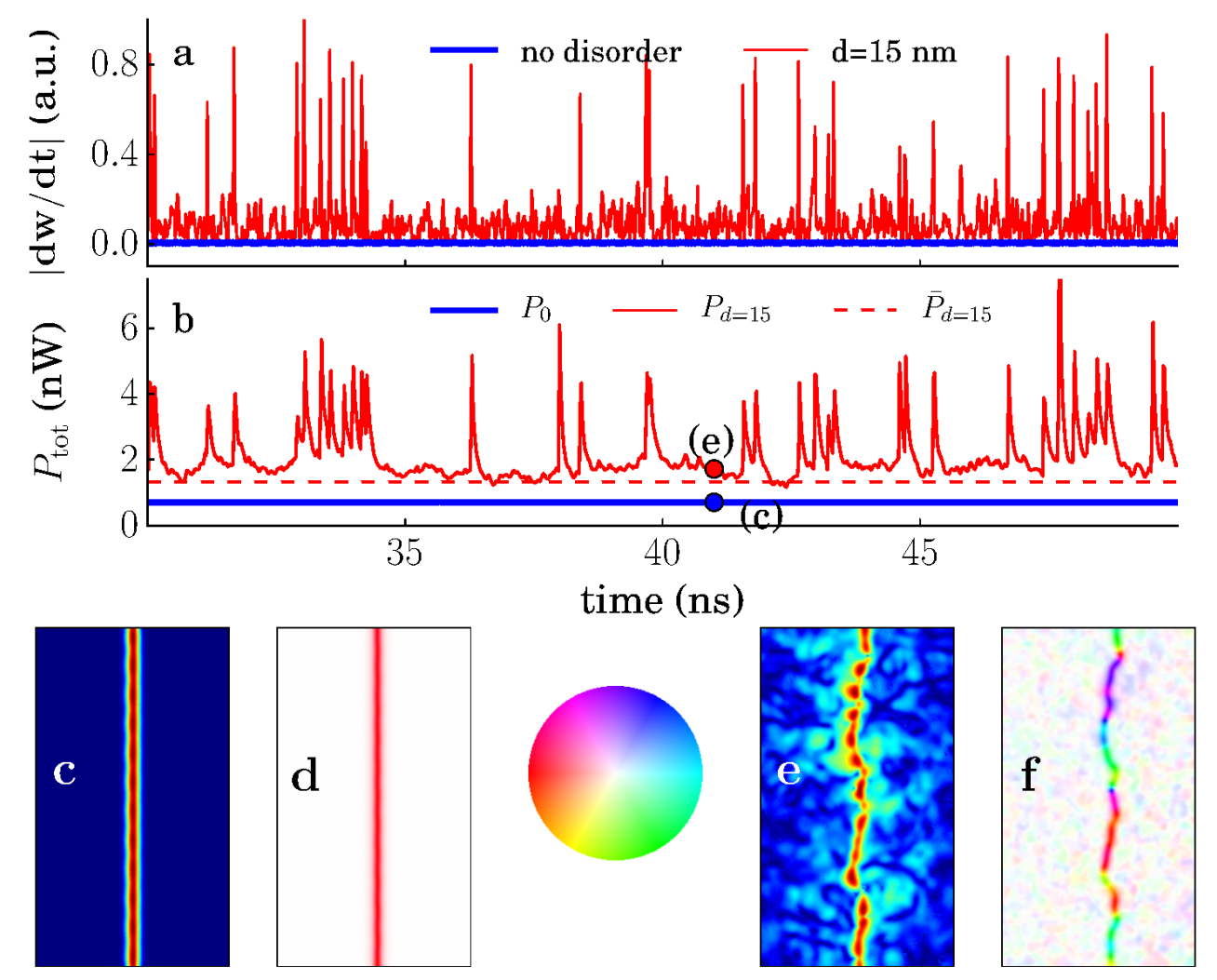

FIGURE 3.17: a. Time evolution of absolute topological charge variation calculated using equation (3.11) for a sample with no disorder (blue line) and another with grain size $d=15 \mathrm{~nm}$ (red line), extracted from simulations with $\alpha=0.015, \mu_{0} \mathrm{H}=140 \mathrm{mT}$ and shown over a time window of $20 \mathrm{~ns}$. b. Time evolution of the total dissipated power calculated using equation (3.3) for the same simulations as in (a), displayed over the same time window. It appears clear the correlation between peaks in dissipated energy and change in topological charge in the case with disorder. The dotted line marks the average dissipation rate $\bar{P}_{d}=\left\langle P_{d}-P_{0}\right\rangle$. c-d Snapshots of dissipated power and magnetization around the DW of the perfect sample at the instant of time indicated in (b) by the blue circle. e-f Snapshots showing the same features for the disordered sample at the instant of time indicated in (b) by the red circle. Hot-cold color gradient represents the amount of local dissipated power in logarithmic scale, in-plane magnetization angle is represented by a color as in the color wheel.

We already mentioned the fact that the extended DW length allows for twisting of the magnetization along the DW. At high fields, this is not the case in a sample without disorder: as can be seen in the snapshot of magnetization in fig. 3.17-d the DW is uniformly magnetized and all the spins are precessing synchronously. This one dimensional-like behaviour is the reason for the good agreement of the analytical model with simulations without disorder observed in fig. 3.13. The introduction 
of disorder, however, yields a much more complex dynamics for the DW, as can be observed in the snapshot of fig. 3.17-f, taken at the same instant and under the same applied field as in fig. 3.17-d. Here the wall appears corrugated and the magnetization twists along the DW length, generating several VBLs.

In the study of Yoshimura and co-authors [59], DMI introduces an in-plane field acting on the wall, affecting the propagation of VBLs by giving different velocities to VBLs of different magnetic charge and causing them to annihilate in couples of same chirality and opposite magnetic charge. This feature is observed also in our simulations, where acceleration, deceleration and annihilation of VBLs are caused by the different energy that VBLs have over grains with different in-plane anisotropy while propagating along a DW, which is also able to curve its profile during dynamics [77, 58]. In addition, the dynamics internal to the DW yields a much higher energy dissipation compared to that of a non-disordered sample: the in-plane anisotropy field, inhomogeneous along the DW length, creates a rich and varied landscape for the VBLs propagating inside the DW and for the DW in general. This results in oscillations and local breathing phenomena of the DW of complicated nature that, overall, generate a constant irradiation of spin waves from the DW. This constant additional dissipated power is absent in films with easy-axis uniformly perpendicular to plane.

Since VBLs have a topological charge, their complex dynamics in DWs propagating in disordered samples can be studied quantitatively by monitoring the time variation of the total topological charge $w$ of our sample.

The absolute value of the variation of topological charge $|d w / d t|$ is shown in fig. 3.17-a as the thick blue curve for a perfect sample and as the thin red curve for a disordered sample with grain size $15 \mathrm{~nm}$. As can be observed, the topological charge is conserved for DW dynamics in a perfect sample, while for samples with grains $\left|\frac{\mathrm{d} w}{\mathrm{~d} t}\right|$ shows peaks corresponding to events of annihilation of couples of VBLs of same topological charge. This is because VBLs are nucleated in couples of opposite topological charge $\pm 1 / 2$ that move in opposite direction, while annihilation takes place between VBLs of same topological charge, so that a net change in $w$ of 1 unit is expected upon annihilation. Such particular dynamics has its origin in the different energy state of a VBL depending on its orientation and the in-plane orientation of the easy-axis of the grain over which it is laying. This grain dependent in-plane anisotropy gives to VBLs grain dependent energy states and velocities, causing acceleration, deceleration and even blocking of VBLs at grain boundaries where a sufficiently high energy barrier is found, generating annihilation events.

The thick blue line in fig. 3.17-b shows the power dissipation over time for the simulations on the perfect sample calculated using (3.3). As can be seen, a constant and low rate of energy dissipation is found, the reason being the 1D-like behaviour of the system. The snapshot of fig. 3.17-c, showing the spatial distribution of the dissipated energy, confirms that energy is being dissipated exclusively inside the DW and homogeneously distributed along its length, since the DW is the only region in which spins are precessing. 
On the other hand, in disordered samples more energy is dissipated during DW motion: the thin red curve in fig. 3.17-b shows the dissipated power in a disordered system with a DW propagating under the same applied field. The red curve describes a non-constant dissipation of power, with peaks corresponding to VBL annihilation events, as confirmed by the agreement with the variation in the topological charge in fig. 3.17-a. It is important to note the offset of the red curve, at about 1.5 $\mathrm{nW}$, making clear the contribution that the complex dynamics internal to the DW, triggered by local non-zero in-plane anisotropy, has on energy dissipation. An additional check of this observation comes from the comparison of the distribution of energy dissipation between a perfect and a disordered sample. Fig. 3.17-e shows that the dissipation of energy in disordered samples takes place not only inside the DW but also in the nearby domains, where spins are precessing due to spin wave emission even when no annihilation event is observed.

The fact that irradiation of energy from the DW to the domains and its subsequent faster dissipation is relevant in DW dynamics, appears also by looking at the variation of tilting angle $\eta$ required by the $1 \mathrm{D}$ model to fit the data when changing damping constant: $\alpha=0.015$ in fig. 3.14-a requires larger $\eta$ to fit simulations for $d=10 \mathrm{~nm}$ compared to $\alpha=0.05$ in fig. 3.14-b, although the samples are exactly the same with identical distribution of $\hat{u}$ and $k_{u}$. Increasing Gilbert's damping, we are increasing the contribution of the applied field to DW velocity, reducing the effect of spin waves and making the 1D approximation more accurate at reproducing the micromagnetic simulation.

We are now able to answer to one of the questions posed at the end of section 3.3, regarding the dependence of DW velocity (fig. 3.11-b) and frequency of annihilation events (fig. 3.12-b) on grain size $d$. Since the distribution of $\hat{u}$ values over grains is aleatory, the intensity and orientation of the in-plane anisotropy in neighbouring grains is uncorrelated. DW motion takes place, as it has been shown, in an incoherent fashion. The non-uniform precession means precession via VBLs propagation, i.e. spins inside the DW precess by $\pi$ sweeps when a VBL passes past them. VBLs have a finite width $\Lambda=\sqrt{A / K}=28.9 \mathrm{~nm}$, which varies depending on the local orientation of $\hat{u}$. This means that the maximum size for coherent precession over which the 1D precessional motion with a uniform and tilted easy-axis can be applied is $\sim 30 \mathrm{~nm}$. When grain size is smaller than this value, the effective tilting of $\hat{u}$ is an average over $\Lambda$ of the different values over grains. Being the disorder uncorrelated, the local average results in a decrease of the in-plane component. Subsequently, VBL will have a more homogeneous propagation and less annihilation events when grain size is smaller than their size $(\sim 30 \mathrm{~nm})$. On the other hand, when grain size is larger than $50 \mathrm{~nm}$, the Bloch line width is smaller than most of the grains. Abrupt changes in local anisotropy become less frequent and the surface density of energy barriers for the propagation of Bloch lines decreases. Consequently, the frequency of VBL annihilation decreases as well with grain size above this value. This explains the 
peak in annihilation events and DW velocity for $d$ around to $30-40 \mathrm{~nm}$. This argument also explains the lower dissipated energy at small $d$ and very high fields, as we will see in next section; VBLs propagate through an effectively more homogeneous medium and reduced effects of breathing and acceleration-deceleration with subsequent annihilation are observed.

\subsubsection{Augmented one dimensional model}

In the analytical model that we have been considering, the system is allowed to dissipate energy only through the displacement and coherent precession of the domain wall, described by the time variation of the two degrees of freedom $q(t)$ and $\phi(t)$, respectively. An additional degree of freedom can be introduced as variation of DW width $\Delta(t)$, but the results do not change considerably because the periodic widening and narrowing of the DW during the dynamics, the so-called breathing, affects the instantaneous velocity $\dot{q}$ and, at high fields and very short precession period, this variation becomes negligible. On the other hand, an important consequence of internal DW dynamics is the emission of spin waves from the wall, as discussed in 3.3. This process transfers energy from the DW to the domains, where it is quickly dissipated. Such mechanism, neglected by the 1D model of Walker and Slonczewski, has been investigated numerically in one dimensional systems, where it has been shown that spin waves emission from the DW can contribute to DW motion when a strong hard-axis anisotropy is present in the system $[84,85,86]$ and also in in-plane magnetized permalloy strips in presence of various forms of disorder [60,87]. In our system, both disorder and the fact that the wall is extended in length play a role.

In what follows we will try to include in the 1D model the additional energy dissipated by the DW in those dynamic process that can not be described by a 1D model. Starting with conservation of energy from LLG equation, eq (1.71):

$$
\frac{\mathrm{d} E}{\mathrm{~d} t}=-\frac{\alpha \mu_{0} M_{s}}{\gamma_{0}} \int_{V}\left(\frac{\mathrm{d} \mathbf{m}}{\mathrm{d} t}\right)^{2} \mathrm{~d} V
$$

we can obtain its one dimensional counterpart that takes into account easy-axis tilting, using eq. (3.6), (3.7) and the DW linear energy $\sigma$ (1.59), where anisotropy constant is described as (3.8), yielding

$$
\begin{gathered}
-2 \mu_{0} M_{s} H_{a} \dot{q}+2 \Delta\left(K \sin 2 \phi-2 K_{u} u_{1} u_{2} \cos 2 \phi\right) \dot{\phi}= \\
=\frac{-2 \alpha \mu_{0} M_{s}}{\gamma_{0}}\left[\frac{\dot{q}^{2}}{\Delta}+\Delta \dot{\phi}^{2}\right] .
\end{gathered}
$$

Within the 1D model framework, we are assuming that the only variation of internal energy comes from the displacement and the rotation of the DW spins and this is balanced by the work done by the viscous forces. Any dynamics taking place in the two domains of our system is neglected. To account for the energy dissipation 
that we observe in simulations with grains and tilted easy-axis, we consider an average constant rate of energy dissipated into the domains rising from the complex dynamics of the incoherently precessing DW. To quantify it, we take the average dissipated power of our simulations with disorder, $\left\langle P_{d}\right\rangle$, corresponding to the average value of the red line in fig. 3.17-b, and we assume that in micromagnetic simulations of the perfect sample energy is dissipated exactly as prescribed by the 1D model. This is a safe assumption at high fields, since we have seen how in that case energy is dissipated exactly as prescribed by the one dimensional model. Then, the term that we need to add to the 1D model equation of conservation of energy is

$$
\bar{P}_{d}=\left\langle P_{d}\right\rangle-\left\langle P_{0}\right\rangle
$$

This value, marked as the dashed red line in fig. 3.17-b, represents the power dissipated by the system in other ways than simple precession of the spins inside the DW. It is dependent on $H_{a}$, since it relies on the dynamics triggered by the precession of the spins inside the DW and needs to be extracted from micromagnetic simulations for every applied field.

Including this term in the energy conservation equation as done elsewhere [88], we have

$$
\begin{aligned}
-2 \mu_{0} M_{s} H_{a} \dot{q}+2 \Delta & \left(K \sin (2 \phi)-2 K_{u} u_{1} u_{2} \cos 2 \phi\right) \dot{\phi}= \\
= & \frac{-2 \alpha \mu_{0} M_{s}}{\gamma_{0}}\left[\frac{\dot{q}^{2}}{\Delta}+\Delta \dot{\phi}^{2}\right]+\frac{\bar{P}_{d}}{t W}
\end{aligned}
$$

Averaging this equation over a period of precession after using expressions (3.6) and (3.7) for $\dot{q}^{2}$ and $\dot{\phi}^{2}$ respectively, we obtain

$$
\begin{array}{r}
\langle v\rangle=\frac{\gamma_{0} \Delta H_{W}}{1+\alpha^{2}}\left[\alpha h_{1}+\frac{1}{\alpha}\left(h_{1}-\sqrt{h_{1}^{2}-1-h_{2}^{2}}\right)\right]+ \\
+\frac{\bar{P}_{d}}{2 \mu_{0} M_{s} H_{a} t W}
\end{array}
$$

Extracting the value of $\bar{P}_{d}$ from micromagnetic simulations with a given grain size $d$ and certain $\alpha$, the augmented model reproduces extremely well the DW velocity of the simulations also for other values of $\alpha$, as can be observed in figure 3.18, proving that the contribution to DW velocity introduced by disorder corresponds to the additional energy dissipated in the domains in form of spin waves.

\subsection{Conclusions}

In this chapter it has been shown how it is possible to reproduce the experimental field-driven DW dynamics in a $\mathrm{CoFeB}$ thin film using the intrinsic Gilbert damping parameter measured experimentally and introducing a realistic crystalline structure 


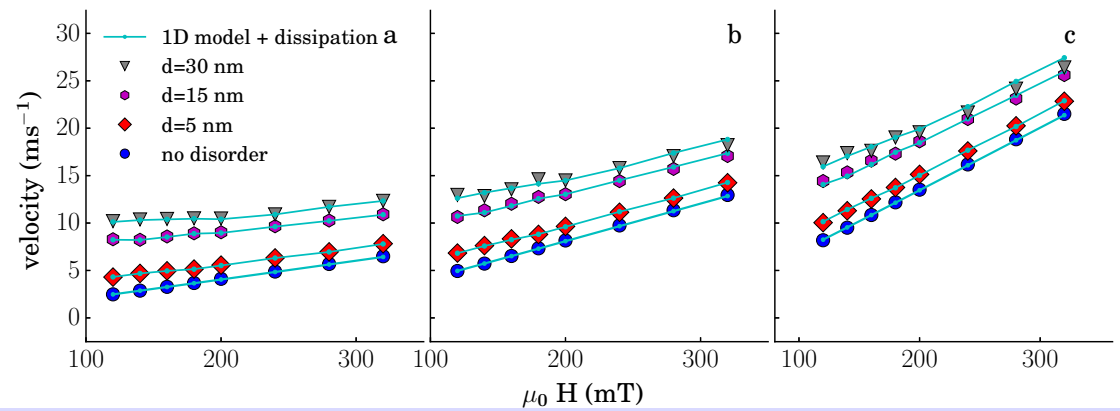

FIGURE 3.18: a. DW velocity versus applied field from simulations with $\alpha=0.015$ without disorder (blue circles) and with different grain sizes $(d=5 \mathrm{~nm}$ red diamonds, $d=15 \mathrm{~nm}$ magenta hexagones, $\mathrm{d}=30 \mathrm{~nm}$ gray down-triangles); Cyan dots show DW velocity computed from $1 \mathrm{D}$ model(3.15) with a realistic $\eta=4.5^{\circ}$ plus dissipation contribution extracted from simulations. Same results for $\alpha=0.03$ and $\alpha=0.05$ are shown in $\mathbf{b}$. and c. respectively, where the dissipation contribution is the one extracted from the simulations in (a) with $\alpha=0.015$

into the system, showing that DW dynamics is qualitatively and quantitatively different from the one of a system with uniform parameters.

We show that the velocity plateau and the viscous regime of DWs in thin films can be attributed to an enhanced energy dissipation in the system generated by extrinsic features, such as defects and granular structure rather than an increased intrinsic damping parameter, representing a dissipation of energy to the lattice. This observation points out the importance of including such features in micromagnetic simulations that aim to realistically reproduce the dynamics of these systems.

We studied the effects of such granular structure in thin films, varying the characteristic size of grains. An optimum diameter of 30-40 nm is found, for which the dissipated energy is maximized and so it is the DW velocity. This optimum size is related to the material parameters through the width of the so-called vertical Bloch lines.

Introducing disorder in simulations gives higher DW velocities in the high field regime while not considerably changing the mobility, and we identified the feature responsible for this velocity increase in the local variation of anisotropy easy-axis orientation. We addressed this problem first introducing an in-plane deviation of the anisotropy easy-axis in the 1D model, proving that this feature was capable of qualitatively reproducing the shift. Secondly, we compared the dissipated power from micromagnetic simulations in a system with disorder with that of a perfectly homogeneous system. We found that the local in-plane anisotropy field is the responsible for oscillations, local breathing phenomena of the DW and VBL annihilations, that overall generate an irradiation of power from the DW to the domains. This irradiated power is constant over time and dependent on system characteristics, such as 
the typical grain size, and independent on the intrinsic Gilbert damping. 


\section{Chapter 4}

\section{Domain wall oscillator as a spin-wave emitter}

\subsection{Introduction}

A spin wave (SW) is a propagating perturbation in the spin texture of a magnetic material in the form of a phase-coherent precession of the magnetic moments [15, $89,90]$. The quanta of spin waves are called magnons and the field investigating the transmission and processing of information mediated by spin waves is termed magnonics. Magnonics offers a promising new route for computing technology because it may overcome the limitations of complementary metal oxide semiconductor (CMOS) technology in terms of scalability and power consumption via a particle-less transmission of information $[7,91,92,93,94,95]$ and by introducing new degrees of freedom encoded in spin waves' transport of angular momentum. Spin waves have short wavelengths at the technologically relevant $\mathrm{GHz}$ - low $\mathrm{THz}$ frequencies, allowing for integration with microwave electronics at the nanoscale [89].

The classical technique used to excite spin waves is via the Oersted field induced around a wire placed on top of the ferromagnet from an ac current flowing through it $[94,92,96]$. This approach allows control of the frequency and wavelength of injected magnons, with the main drawback that the antenna width sets a lower bound for wavelength and limits the scalability of the device. An alternative way of inducing linear excitation of short-wavelength spin waves is through the uniform microwave excitation of a inhomogeneous magnetization texture $[97,98]$. The conversion of electron-carried angular momentum into magnons [99, 100, 101] and vice versa $[102,96]$ allows for the exploitation of spintronics phenomena for the generation and detection of spin waves at the nanoscale and the embedding of magnonic circuitry in electronic-based devices. This novel field is called magnon-spintronics. Here, spin wave generation can be achieved by various localized excitations, such as electric field control of the magnetostrictive properties of materials [103, 104, 105] and spin transfer torque $[106,107,108]$ (STT), generated either by a spin-polarized current flowing through a nanocontact $[99,100]$ or via the spin current originated by the flow of charge current through an adjacent non-magnetic metal with large spin-orbit coupling [101, 109, 110, 111]. 
It is known that a domain wall can emit spin waves during its motion $[112,84,85$, 113,114 ] or under microwave linear excitation [98]; the use of an oscillating domain wall as a tuneable spin wave emitter excited by an alternate current has been proposed by Van de Wiele and colleagues [115]. In their work, a strong pinning of the DW is achieved via ferromagnetic-ferroelectric coupling, and an ac current is used to generate DW oscillations that excite propagation of SWs in adjacent domains at an angle of $45^{\circ}$ with respect to the magnetization orientation. The use of a DW permits SW excitation at wavelengths much shorter than can be achieved with common antennas and the change in ac frequency can, to some extent, regulate the emitted frequency. However, the realization of such a device presents some limitations: high current densities are required, the fabrication of hybrid ferromagnetic-ferroelectric structures is difficult, and the propagation of SWs in the $45^{\circ}$ magnetized domains is non-trivial.

It was shown both analytically and numerically [116, 117, 118, 119] that a DW pinned at a constriction in a perpendicularly magnetized nanowire could be led to self-sustained full in-plane rotation by the STT exerted on it by the injection of a low in-plane dc current while remaining pinned at a localized pinning site, thus creating a DW-based oscillator with frequencies in the GHz range, tuneable via the applied current intensity.

In this chapter we reconsider the DW oscillator set-up, schematically represented in Fig. 4.9-a, and investigate, using micromagnetic simulations, the emission of SWs generated by such localized magnetization precession in a nanowire. By selecting an adequate wire width and constriction geometry, we can achieve a wide operating window in which we observe DW rotation at a current-dependent frequency $\left(f_{D W}\right)$ that leads to unidirectional emission of SWs in the direction of electrons' flow at odd harmonics of $f_{D W}$. Because the SW frequency is a multiple of $f_{D W}$, the frequency of the emitted SWs can also be tuned by changing current intensity. Moreover, through the application of an external field opposing the force exerted on the DW by the current, the device operating window is extended and, at the same time, the symmetric dispersion relation for SWs in the two antiparallel domains is naturally split, which allows us to selectively propagate different harmonics along each domain. We identify the DW's in-plane stray field as the main factor responsible for SW excitation, whereas the unidirectionality is due to the asymmetric position of the DW below the geometrical constriction. This new concept of SW emitter has the attractive features of high coherence, a tuneable frequency up to tens of $\mathrm{GHz}$ and low power consumption (typical current of a few $\mu \mathrm{A}$ ) by simply exploiting the stray field induced by geometrical patterning.

In section 4.2 the main tools used for this work are introduced, namely, micromagnetic model for current driven magnetization dynamics, its one dimensional model equivalent, where equations of pinned domain wall dynamics are derived, and the fundamentals of dipolar-exchange spin waves propagation dynamics.

In section 4.3 the path towards an efficient spin wave emitter is described: the 
computation of non-uniform current distribution in the ferromagnetic wire, the effect of wire cross section and notch shape on domain wall precession and the effects of geometry on the domain wall dynamics. Finally, in section 4.4 a spin wave emitter setup is studied thoroughly: the emission of spin waves and its origin are analysed and their dependence on the applied current is studied as well as the effect of the application of a static external field.

\subsection{Theoretical background}

\subsubsection{Current induced domain wall motion}

When a current density $\mathbf{j}_{\mathrm{c}}$ crosses a $180^{\circ}$ domain wall in a metallic ferromagnet, the spin $\mathbf{s}$ of the conduction electron exerts an exchange torque on the localized spins of the wall [106]. More generally, a number of effects rise from the interplay between spin dependent transport properties and magnetization dynamics in ferromagnets [108]. The underlying cause is the torque generated by exchange interaction between conducting electrons and and magnetization electrons, the so-called s-d exchange torque [106]. The torque exerted by a current density $\mathbf{j}_{\mathrm{c}}$ on the magnetization $\mathbf{M}$ is described by the so-called spin transfer torque (STT)

$$
\tau_{\mathrm{STT}}=(\mathbf{u} \cdot \nabla) \mathbf{m}+\beta \mathbf{m} \times[(\mathbf{u} \cdot \nabla) \mathbf{m}],
$$

where the spin-drift velocity $\mathbf{u}$ is

$$
\mathbf{u} \stackrel{\text { def }}{=} \frac{\mathbf{j}_{c}}{1+\beta^{2}} \frac{P \mu_{B}}{e M_{s}}
$$

Here, $e$ is the negative electron charge, $\mu_{B}$ is the Bohr magneton, $\gamma_{0}=2.21 \times$

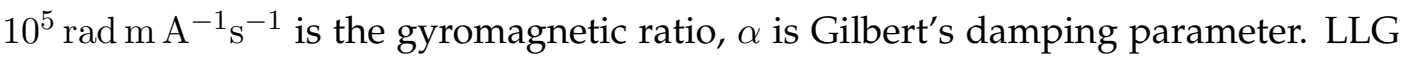
equation of magnetization dynamics under the effect of a flowing current density reads

$$
\frac{\mathrm{d} \mathbf{m}}{\mathrm{d} t}=-\gamma_{0} \mathbf{m} \times \mathbf{H}_{\mathrm{eff}}+\alpha \mathbf{m} \times \frac{\mathrm{d} \mathbf{m}}{\mathrm{d} t}-(\mathbf{u} \cdot \nabla) \mathbf{m}+\beta \mathbf{m} \times[(\mathbf{u} \cdot \nabla) \mathbf{m}],
$$

and its explicit form is

$$
\begin{aligned}
\frac{\mathrm{d} \mathbf{m}}{\mathrm{d} t}= & -\frac{\gamma_{0}}{1+\alpha^{2}}\left[\mathbf{m} \times \mathbf{H}_{\mathrm{eff}}+\alpha \mathbf{m} \times\left(\mathbf{m} \times \mathbf{H}_{\mathrm{eff}}\right)\right] \\
& -\frac{1+\alpha \beta}{1+\alpha^{2}}(\mathbf{u} \cdot \nabla) \mathbf{m}+\frac{\beta-\alpha}{1+\alpha^{2}} \mathbf{m} \times[(\mathbf{u} \cdot \nabla) \mathbf{m}] .
\end{aligned}
$$


The one dimensional model equations of motion derived from LLG equation (4.3) are now

$$
\begin{aligned}
\dot{\phi}+\alpha \frac{\dot{q}}{\Delta} & =\gamma_{0} H_{a}+\beta \frac{u}{\Delta} \\
\frac{\dot{q}}{\Delta}-\alpha \dot{\phi} & =\gamma_{0} \frac{H_{K}}{2} \sin 2 \phi+\frac{u}{\Delta}
\end{aligned}
$$

In their explicit form they read:

$$
\begin{aligned}
\dot{q} & =\frac{\gamma_{0} \Delta}{1+\alpha^{2}}\left(\alpha H_{a}+\frac{H_{K}}{2} \sin 2 \phi\right)+\frac{1+\alpha \beta}{1+\alpha^{2}} u \\
\dot{\phi} & =\frac{\gamma_{0}}{1+\alpha^{2}}\left(H_{a}-\alpha \frac{H_{K}}{2} \sin 2 \phi\right)+\frac{\beta-\alpha}{1+\alpha^{2}} \frac{u}{\Delta}
\end{aligned}
$$

where we are neglecting the dynamical variation of DW width $\Delta$.

$$
\dot{\Delta}=\frac{12 \gamma_{0}}{\pi^{2} \alpha M_{s}}\left[\frac{A}{\Delta}-\Delta\left(K_{0}+K \sin ^{2} \phi\right)\right]
$$

It is useful to schematically represent the contribution to the magnetization dynamics of the various terms in equations (4.7),(4.8). In order to do so, we recall that in the 1D model formulation, our magnetization has the profile (1.31).

$$
\theta(x, t)=2 \arctan \left[\exp \left(\frac{x-q(t)}{\Delta}\right)\right]
$$

which yields

$$
\dot{\theta}=-\sin \theta \frac{\dot{q}}{\Delta} .
$$

This means that at the DW center, where the magnetic spin is in-plane $(\theta=\pi / 2)$, the out of plane torque reads:

$$
\dot{\theta}=\tau_{\theta}=-\frac{\dot{q}}{\Delta} \hat{\mathbf{z}}
$$

and, of course, the in-plane torque is $\dot{\varphi}=\tau_{\varphi}=\dot{\phi}$.

Since $\theta=0$ when $\mathbf{m} \| \hat{\mathbf{z}}$, the torque $\tau_{\theta}$ that brings the spins from in-plane to positive out-of-plane, giving a positive velocity to the $\mathrm{DW}$, gives a negative variation of $\theta$. We are assuming a Bloch wall configuration, for which $H_{K}=M_{s}\left(N_{y}-N_{x}\right)<0$, and $u \geq 0$.

The contributions to the out of plane torque acting on the DW, originating from the application of an external field, the shape anisotropy and the STT from an external current are shown in table 4.1, while the contributions of the same interactions to the in-plane torque are presented in table 4.2. As we can observe, the applied field and the external current have a fixed contribution, independent on the DW orientation, while the shape anisotropy torques change sign when changing quadrant, giving rise to back-and-forth oscillations of the DW and to a non-uniform in-plane rotation. Such features of domain wall dynamics, introduced by shape anisotropy, appear also in the pinned rotation regime that we will study in detail in next section. 


\begin{tabular}{c|c|c|c} 
quadrant & $\alpha \gamma_{0} H_{a}$ & $\frac{1}{2} \gamma_{0} H_{K} \sin 2 \phi$ & $(1+\alpha \beta) \frac{u}{\Delta}$ \\
\hline I & $\operatorname{sign}\left(H_{a}\right) \hat{\mathbf{z}}$ & $-\hat{\mathbf{z}}$ & $\hat{\mathbf{z}}$ \\
II & $\operatorname{sign}\left(H_{a}\right) \hat{\mathbf{z}}$ & $\hat{\mathbf{z}}$ & $\hat{\mathbf{z}}$ \\
III & $\operatorname{sign}\left(H_{a}\right) \hat{\mathbf{z}}$ & $-\hat{\mathbf{z}}$ & $\hat{\mathbf{z}}$ \\
IV & $\operatorname{sign}\left(H_{a}\right) \hat{\mathbf{z}}$ & $\hat{\mathbf{z}}$ & $\hat{\mathbf{z}}$ \\
\hline
\end{tabular}

TABLE 4.1: Contribution to the out-of-plane torque $-\left(1+\alpha^{2}\right) \dot{\theta}$ at the center of the DW where $\theta=\pi / 2$, depending on the in-plane orientation of the DW.

\begin{tabular}{c|c|c|c} 
quadrant & $\gamma_{0} H_{a}$ & $-\frac{1}{2} \alpha \gamma_{0} H_{K} \sin 2 \phi$ & $(\beta-\alpha) \frac{u}{\Delta}$ \\
\hline I & $\operatorname{sign}\left(H_{a}\right)$ & $-\operatorname{sign} H_{K}$ & $\operatorname{sign}(\beta-\alpha)$ \\
II & $\operatorname{sign}\left(H_{a}\right)$ & $\operatorname{sign} H_{K}$ & $\operatorname{sign}(\beta-\alpha)$ \\
III & $\operatorname{sign}\left(H_{a}\right)$ & $-\operatorname{sign} H_{K}$ & $\operatorname{sign}(\beta-\alpha)$ \\
IV & $\operatorname{sign}\left(H_{a}\right)$ & $\operatorname{sign} H_{K}$ & $\operatorname{sign}(\beta-\alpha)$ \\
\hline
\end{tabular}

TABLE 4.2: Sign, $1=$ Anti-clockwise (ACW) and -1=Clockwise (CW), of the in-plane torque $\dot{\phi}$ at the center of the DW, depending on the in-plane orientation.

In a freely propagating DW driven by current, with $u>0$ and $H_{a}=0$, we have

$$
\left(1+\alpha^{2}\right) \dot{\phi}=-\frac{\alpha \gamma_{0} H_{K}}{2} \sin 2 \phi+(\beta-\alpha) \frac{u}{\Delta}
$$

and a stationary DW orientation is attained when $\dot{\phi}=0$, in which case we have

$$
\begin{aligned}
(\beta-\alpha) \frac{u}{\Delta} & =\frac{\alpha \gamma_{0} H_{K}}{2} \sin 2 \phi \\
u & =\frac{\alpha \Delta \gamma_{0} H_{K}}{2(\beta-\alpha)} \sin 2 \phi \\
u & \leq \frac{\alpha \Delta \gamma_{0}\left|H_{K}\right|}{2|\beta-\alpha|}=\frac{\gamma_{0} \Delta H_{W}}{|\beta-\alpha|}
\end{aligned}
$$

This threshold defines a Walker current

$$
j_{W}=\frac{e M_{s}}{P \mu_{B}} \frac{\gamma_{0} \Delta H_{W}}{|\beta-\alpha|},
$$

which is the equivalent of the Walker field for field driven dynamics, separating a rigid regime from a precessional regime, depending on the competition between the restoring torque due to shape anisotropy and the precession of the DW induced by the application of an in-plane current. However, differences exist with field driven dynamics. By looking at tables 4.1 and 4.2, we can see that the competition of STT with the shape anisotropy torque can be constructive or destructive, depending on the sign of $\beta-\alpha$. In the purely adiabatic case, $\beta=0$, starting with a relaxed Bloch configuration for the DW, for $j<j_{W}$ the domain wall reaches in a finite time an equilibrium configuration where STT and shape anisotropy torques balance each other and no motion takes place. This feature of adiabatic STT-driven DW motion is called intrinsic pinning and depends directly on shape anisotropy field $H_{K}$. In 
the case of $\beta=\alpha$, there is no contribution to the in-plane torque due to current and $\dot{q}=u, \dot{\phi}=0$ for any value of $u$.

Since we are interested in the DW precession frequency, a non-adiabaticity $\beta=$ $2 \alpha$ is considered.

From equation (4.8) without external field contribution:

$$
\dot{\phi}=-\frac{\alpha \gamma_{0} H_{K}}{2\left(1+\alpha^{2}\right)} \sin 2 \phi+\frac{\beta-\alpha}{1+\alpha^{2}} \frac{u}{\Delta}
$$

In the case of a low shape anisotropy $H_{K} \simeq 0$, perfectly adiabatic torque $\beta=0$, and $j \gg j_{W}$ we have

$$
\dot{\phi}=-\frac{\alpha}{1+\alpha^{2}} \frac{u}{\Delta},
$$

which means that the rotation of a propagating DW takes place with a frequency proportional to $\alpha$. In the next section we will see that a pinned domain wall can rotate at much higher frequencies.

\subsubsection{One dimensional theory of pinned domain wall oscillator}

A rigorous approach to introduce pinning in the one dimensional model is found in [118]; there, pinning is introduced in the system as a localised parabolic potential well with stiffness $k\left(\mathrm{~J} \mathrm{~m}^{-2}\right)$ and width $l$ :

$$
V_{p}(q)= \begin{cases}\frac{k}{2}(q-p)^{2} & \text { if }|q-p|<l \\ 0 & \text { else. }\end{cases}
$$

The effective field due to the geometrical constriction is then

$$
H_{p}(q)=-\left(2 \mu_{0} M_{s} L_{y} L_{z}\right)^{-1} \partial V_{p}(q) / \partial q=-\frac{k(q-p)}{2 \mu_{0} M_{s} L_{y} L_{z}}
$$

as long as $|p-q|<l$.

The general dynamic equations (4.7) and (4.8) with this new contribution are now

$$
\begin{aligned}
& \dot{q}=\frac{\gamma_{0} \Delta}{1+\alpha^{2}}\left(\alpha\left(H_{a}+H_{p}(q)\right)+\frac{H_{K}}{2} \sin 2 \phi\right)+\frac{1+\alpha \beta}{1+\alpha^{2}} u \\
& \dot{\phi}=\frac{\gamma_{0}}{1+\alpha^{2}}\left(H_{a}+H_{p}(q)-\alpha \frac{H_{K}}{2} \sin 2 \phi\right)+\frac{\beta-\alpha}{1+\alpha^{2}} \frac{u}{\Delta} .
\end{aligned}
$$

The torque exerted on the DW by the geometrical confinement is mostly dependent on the position $q$ with respect to the pinning center $p$ and only weakly dependent on the DW in-plane orientation. Oscillation is then attained as a competition between the shape anisotropy and constriction torque, one dependent on DW position the other on DW orientation, in a non-equilibrium configuration introduced by the applied current. 
From eq. (4.17), we see that for

$$
u<\frac{\gamma_{0} \Delta}{2(1+\alpha \beta)} H_{p}^{\max }=u_{\mathrm{dep}}
$$

there exists a position $\bar{q}$ such that $\gamma_{0} \Delta \alpha H_{p}(\bar{q})=-(1+\alpha \beta) u$ and equation (4.17) reduces to

$$
\dot{q}=\frac{\gamma_{0} \Delta}{1+\alpha^{2}} \frac{H_{K}}{2} \sin 2 \phi
$$

which describes a back and forth oscillation of the DW below the pinning site if $\bar{q}<l-\gamma_{0} \Delta\left|H_{K}\right|\left(1+\alpha^{2}\right)^{-1}$ is sufficiently away from the edge of the potential well. However, this condition does not guarantee an equilibrium in the in plane rotation. In fact, substituting the same condition for $q=\bar{q}$ in (4.18), we obtain

$$
\dot{\phi}=-\frac{u}{\alpha \Delta}-\frac{\alpha \gamma_{0}}{2\left(1+\alpha^{2}\right)} H_{K} \sin 2 \phi
$$

which describes a uniform precession with the superposition of a weak ellipticity given by the shape anisotropy.

The system can reach an equilibrium configuration when both (4.17) and (4.18) equal zero. If we select a current such that $u<u_{\text {dep }}$, we can assume that the DW will stay pinned and move around a position $\bar{q}$ where pinning strength and STT equal each other.

$$
\begin{aligned}
\frac{\gamma_{0} \Delta}{1+\alpha^{2}}\left(\alpha H_{p}(\bar{q})+\frac{H_{K}}{2} \sin 2 \phi\right)+\frac{1+\alpha \beta}{1+\alpha^{2}} u=0, \\
\frac{\gamma_{0}}{1+\alpha^{2}}\left(H_{p}(\bar{q})-\alpha \frac{H_{K}}{2} \sin 2 \phi\right)+\frac{\beta-\alpha}{1+\alpha^{2}} \frac{u}{\Delta}=0 .
\end{aligned}
$$

We multiply equation (4.22) by $\alpha \Delta$ and subtract it from equation (4.21) to obtain

$$
\frac{\gamma_{0} \Delta H_{K}}{2\left(1+\alpha^{2}\right)} \sin 2 \phi\left(1+\alpha^{2}\right)+\frac{1+\alpha \beta}{1+\alpha^{2}} u-\frac{\alpha \Delta(\beta-\alpha) u}{\left(1+\alpha^{2}\right) \Delta}=0
$$

yielding the condition:

$$
u=-\frac{\gamma_{0} \Delta H_{K}}{2} \sin 2 \phi
$$

This means that equilibrium is attained $(\dot{q}=\dot{\phi}=0)$ if $\sin 2 \phi^{*}=-\frac{2 u}{\gamma_{0} \Delta H_{K}}$, which means

$$
\phi^{*}=\frac{1}{2} \arcsin \left(-\frac{2 u}{\gamma_{0} \Delta H_{K}}\right)
$$

only possible if $|u| \leq\left|\frac{\gamma_{0} \Delta H_{K}}{2}\right|=u_{\text {rot }}$.

Within this framework, pinned DW rotation is attained for velocities $u$ in the range:

$$
u_{\mathrm{rot}}=\frac{\gamma_{0} \Delta H_{K}}{2}<J_{c}<\frac{\alpha \gamma_{0} \Delta H_{p}^{\max }}{1+\alpha \beta}=u_{\mathrm{dep}}
$$


equivalent to a range of applied current density $J_{c}$ :

$$
J_{\mathrm{rot}}=\frac{e M_{s}}{P \mu_{B}} \frac{\gamma_{0} \Delta H_{K}}{2}<J_{c}<\frac{e M_{s}}{P \mu_{B}} \frac{\alpha \gamma_{0} \Delta H_{p}^{\max }}{1+\alpha \beta}=J_{\mathrm{dep}} .
$$

\subsubsection{Dipolar-exchange spin wave theory}

The second fundamental process over which we base our study is spin wave (SW) emission and propagation.

The first theoretical tool that we need is the spin waves dispersion relation for our system, linking the admitted wave number and frequencies for propagating SW. The most direct way to obtain this equation is assuming small plane wave perturbation of a uniform magnetic state and linearise LLG equation of magnetization dynamics neglecting dissipation.

Let's assume that we have a thin magnetic film of thickness $t$ and lateral dimensions $L_{x}, L_{y}>t$. The film has a strong out-of-plane anisotropy, of interfacial or crystalline nature, such that it is uniformly magnetized out of plane. We introduce a perturbation in the magnetization in form of a plane wave

$$
\begin{aligned}
\mathbf{m} & =\mathbf{m}_{0}+\delta \mathbf{m} \\
\mathbf{m}_{0} & =\hat{\mathbf{z}} \\
\delta \mathbf{m} & =e^{i(\mathbf{k} \cdot \mathbf{x}-\omega t)}\left(\delta m_{x} \hat{\mathbf{x}}+\delta m_{y} \hat{\mathbf{y}}\right)
\end{aligned}
$$

where we are including the effect of the dipolar field in the effective anisotropy $k_{\mathrm{eff}}=$ $k_{u}-\frac{\mu_{0} M_{s}^{2}}{2}$ and we assume that the perturbation along $\hat{\mathbf{z}}$ is negligible.

We consider the LLG equation (1.6) without damping

$$
\dot{\mathbf{m}}=-\gamma_{0} \mathbf{m} \times \mathbf{H}_{\text {eff }}
$$

where we are using $\dot{\mathbf{m}}$ for the time derivative of $\mathbf{m}$, and we write the effective field as the sum of the various contributions:

$$
\mathbf{H}_{\mathrm{eff}}=\mathbf{H}_{\mathrm{Z}}+\mathbf{H}_{\mathrm{ex}}+\mathbf{H}_{\mathrm{an}, \|}+\mathbf{H}_{\mathrm{an}, \perp}
$$

where

$$
\begin{aligned}
\mathbf{H}_{\mathrm{Z}} & =H \hat{\mathbf{z}} \\
\mathbf{H}_{\mathrm{ex}} & =\frac{2 A}{\mu_{0} M_{s}} \nabla^{2} \mathbf{m}=\frac{2 A}{\mu_{0} M_{s}} \nabla^{2} \delta \mathbf{m}=-\frac{2 A}{\mu_{0} M_{s}}|\mathbf{k}|^{2} \delta \mathbf{m} \\
\mathbf{H}_{\mathrm{an}, \|} & =\frac{2 k_{\mathrm{eff}}}{\mu_{0} M_{s}} \mathbf{u}(\mathbf{m} \cdot \mathbf{u})=\frac{2 k_{\mathrm{eff}}}{\mu_{0} M_{s}} \hat{\mathbf{z}} \\
\mathbf{H}_{\mathrm{an}, \perp} & =\frac{2 k_{\perp}}{\mu_{0} M_{s}} \mathbf{v}(\mathbf{m} \cdot \mathbf{v})=\frac{2 k_{\perp}}{\mu_{0} M_{s}} \delta \mathbf{m}_{y} \hat{\mathbf{y}} .
\end{aligned}
$$


The effective field (1.10) reads then

$$
\begin{aligned}
\mathbf{H}_{\mathrm{eff}}= & -\frac{2 A}{\mu_{0} M_{s}}|\mathbf{k}|^{2} \delta m_{x} e^{i(\mathbf{k} \cdot \mathbf{x}-\omega t)} \hat{\mathbf{x}}+\left(-\frac{2 A}{\mu_{0} M_{s}}|\mathbf{k}|^{2}+\frac{2 k_{\perp}}{\mu_{0} M_{s}}\right) \delta m_{y} e^{i(\mathbf{k} \cdot \mathbf{x}-\omega t)} \hat{\mathbf{y}} \\
& +\left(\frac{2 k_{\mathrm{eff}}}{\mu_{0} M_{s}}+H\right) \hat{\mathbf{z}} .
\end{aligned}
$$

Note that we are including in the system the existence of a transverse shape anisotropy $k_{\perp}=\frac{\mu_{0} M_{s}^{2}}{2}\left(N_{y}-N_{x}\right)$ in the $\hat{\mathbf{y}}$ direction in order to consider the case of a narrow strip $L_{y}<<L_{x}$. We have:

$\mathbf{m} \times \mathbf{H}_{\text {eff }}=\left|\begin{array}{ccc}\mathbf{x} & \mathbf{y} & \mathbf{z} \\ \delta m_{x} e^{i(\mathbf{k} \cdot \mathbf{x}-\omega t)} & \delta m_{y} e^{i(\mathbf{k} \cdot \mathbf{x}-\omega t)} & 1 \\ -\frac{2 A}{\mu_{0} M_{s}}|\mathbf{k}|^{2} \delta m_{x} e^{i(\mathbf{k} \cdot \mathbf{x}-\omega t)} & \left(-\frac{2 A}{\mu_{0} M_{s}}|\mathbf{k}|^{2}+\frac{2 k_{\perp}}{\mu_{0} M_{s}}\right) \delta m_{y} e^{i(\mathbf{k} \cdot \mathbf{x}-\omega t)} & \frac{2 k_{\mathrm{eff}}}{\mu_{0} M_{s}}+H\end{array}\right|$

whereas, deriving eq. (4.25), and using (4.26) and (4.27), we get

$$
\dot{\mathbf{m}}=-i \omega \delta \mathbf{m} .
$$

The linearised LLG equation reads:

$$
\begin{aligned}
& -i \omega e^{i(\mathbf{k} \cdot \mathbf{x}-\omega t)} \delta m_{x}=-\gamma_{0} e^{i(\mathbf{k} \cdot \mathbf{x}-\omega t)} \delta m_{y}\left(B(\mathbf{k})-\frac{2 k_{\perp}}{\mu_{0} M_{s}}\right) \\
& -i \omega e^{i(\mathbf{k} \cdot \mathbf{x}-\omega t)} \delta m_{y}=\gamma_{0} e^{i(\mathbf{k} \cdot \mathbf{x}-\omega t)} \delta m_{x} B(\mathbf{k})
\end{aligned}
$$

where

$$
B(\mathbf{k})=\frac{2}{\mu_{0} M_{s}}\left(k_{\mathrm{eff}}+A|\mathbf{k}|^{2}\right)+H,
$$

and we are assuming there is no variation in the $\mathbf{z}$ component. Therefore

$$
\begin{aligned}
-i \omega \delta m_{x}+\gamma_{0} \delta m_{y}\left(B-\frac{2 k_{\perp}}{\mu_{0} M_{s}}\right) & =0 \\
-i \omega \delta m_{y}-\gamma_{0} \delta m_{x} B & =0
\end{aligned}
$$

This homogeneous linear system has non-trivial solutions if and only if the determinant of its associated matrix is zero, i.e.:

$$
\left|\begin{array}{cc}
i \omega & \gamma_{0}\left(B-\frac{2 k_{\perp}}{\mu_{0} M_{s}}\right) \\
\gamma_{0} B & i \omega
\end{array}\right|=-\omega^{2}+\gamma_{0}^{2} B(\mathbf{k})\left(B(\mathbf{k})-\frac{2 k_{\perp}}{\mu_{0} M_{s}}\right)=0,
$$

which gives the dispersion relation:

$$
\omega=\left|\gamma_{0}\right| \sqrt{B(\mathbf{k})\left(B(\mathbf{k})-\frac{2 k_{\perp}}{\mu_{0} M_{s}}\right)} .
$$


Dropping the weak shape anisotropy term we have the simplified version of the dispersion relation:

$$
\omega=\gamma_{0}\left[\frac{2}{\mu_{0} M_{s}}\left(k_{\mathrm{eff}}+A|\mathbf{k}|^{2}\right)+H\right]
$$

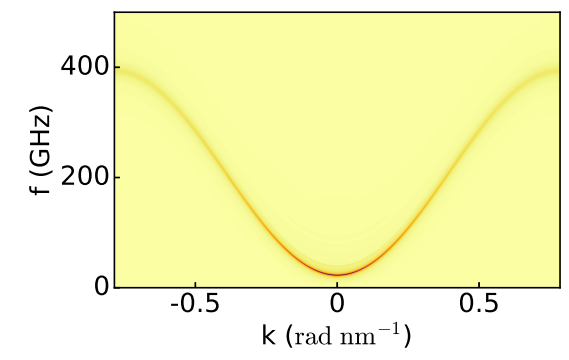

a

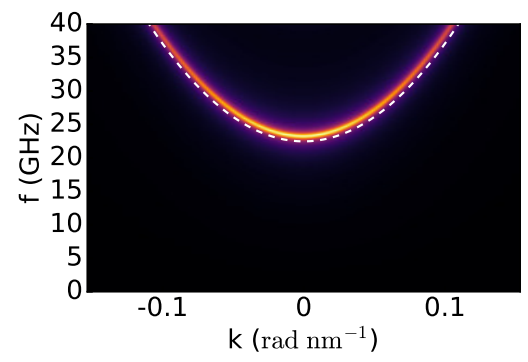

b

FIGURE 4.1: a. $f-k$ diagram extracted from micromagnetic simulations where spin waves are excited with a sinc pulse (4.43). b. Detail of the diagram with analytical dispersion relation (4.41) superimposed as dashed line. Material parameters used are those of CoFeB studied in chapter 3.3, with Gilbert damping parameter $\alpha=0$.

The dispersion relation for SW in our system (4.41) is the main analytical tool to understand our results on SW emission from micromagnetic simulation. To establish a correspondence between our system and the theory, we extract the spectrum of SW propagation along the $\hat{\mathbf{x}}$ direction in a strip of dimensions $8192 \times 60 \times 1 \mathrm{~nm}$. We excite all modes applying a space and time varying external field along the $\hat{\mathbf{y}}$ direction as:

$$
\mathbf{H}_{\mathrm{ext}}=F(x, y, t) \hat{\mathbf{y}}
$$

with

$$
F(x, t)=\mathcal{F} \frac{\sin \left(2 \pi f_{c} t^{\prime}\right)}{2 \pi f_{c} t^{\prime}} \frac{\sin \left(k_{c} x^{\prime}\right)}{k_{c} x^{\prime}}
$$

where $\mu_{0} \mathcal{F}=0.01 \mathrm{~T}, x^{\prime}=x-x_{0}, t^{\prime}=t-t_{0}$ and $\left(x_{0}, y_{0}\right)$ was the center of the stripe, $t_{0}=50 \mathrm{ps}$. The cutoff values $f_{c}=500 \mathrm{GHz}$ and $k_{c}=\pi / 4 \times 10^{9} \mathrm{rad} \cdot \mathrm{m}^{-1}$ are determined by the time-step and spatial discretization. Gilbert's damping is set to zero throughout the whole sample and absorbing boundary conditions are applied at the x-edges of the strip to avoid reflection. Material parameters of $\mathrm{CoFeB}$ thin films studied in chapter 3 ( 3.3) are used. The 2D fourier transform of the values of magnetization taken at the center of the strip over a time of $5 \mathrm{~ns}$ with $\Delta t=1 \mathrm{ps}$ and a spatial range of $2048 \mathrm{~nm}$ discretized in cells of $\Delta x=4 \mathrm{~nm}$ produces the $f-k$ diagram in figure 4.1 .

The use of a sinc pulse (4.43) is a common technique in micromagnetic simulations $[120,121,122,123]$ to obtain rapidly a large frequency spectrum of spin wave 


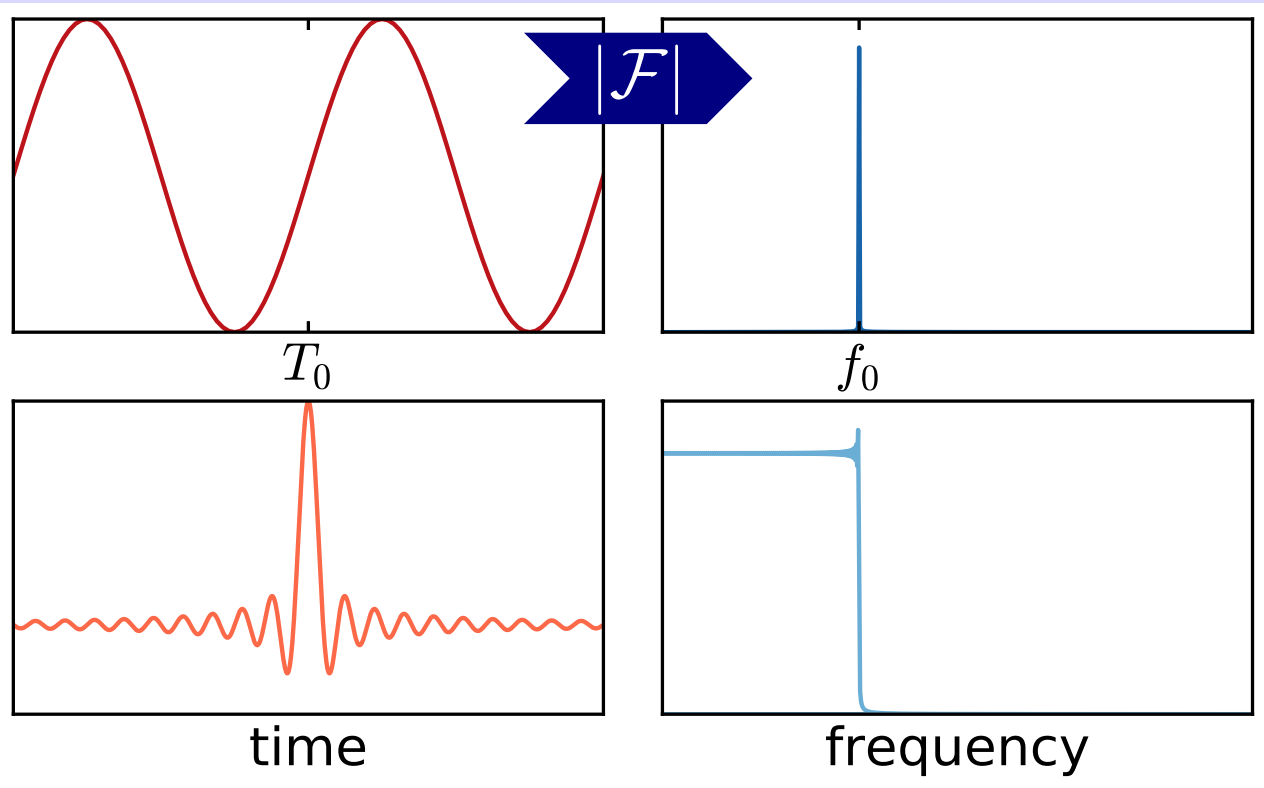

FIGURE 4.2: Difference in the frequency spectrum between an harmonic and a sinc excitation.

emission. In fact, while an harmonic excitation $\sin \left(2 \pi f_{0} t\right)$ excites exclusively the frequency $f_{0}$, the Fourier transform of $\frac{\sin \left(2 \pi f_{c} t\right)}{2 \pi f_{c} t}$ is a step function, non-zero between 0 and $f_{0}$ (see fig. 4.2), which means that we are exciting equally all frequencies up to $f_{0}$.

As can be observed in fig 4.1, the analytical expression for the dispersion relation in our system (4.41) describes well the principal branch of the diagram for $k \leq 1.5 \times 10^{8}$, with a slight underestimation of the frequencies. This corresponds to frequencies of up to $50 \mathrm{GHz}$ and it is sufficient for our study.

\subsection{Towards spin wave emitter}

In a domain wall oscillator where the pinning is obtained via a geometrical constriction, current density is expected to be highly non-uniform around the notch. Since STT is proportional to current density (4.1), it is important to properly evaluate the current density spatial distribution to describe accurately the current driven magnetization dynamics. The space varying current distribution adds complexity to the study, making the driving force position-dependent. This feature adds to the position-dependent confinement force of the geometrical pinning.

\subsubsection{Non-uniform current distribution}

The planar current distribution is computed before resolving micromagnetic dynamics and kept constant throughout the micromagnetic simulation. In the following we 
briefly describe the tool used to numerically compute the spatial distribution of current density.

Two regions of the sample are defined as the input and output contacts. In these regions a scalar potential $\rho$ is set constant to the two different values $\rho^{+}$and $\rho^{-}$. After initialization the scalar potential is diffused over the sample outside the contacts

$$
\frac{\partial \rho}{\partial t}-\nabla^{2} \rho=0
$$

using a successive-over-relaxation algorithm (SOR) implemented with finite difference until homogeneity is achieved with an error $\epsilon<10^{-9}$.

$$
\begin{aligned}
\rho^{k+1} & =\omega \tilde{\rho}^{k+1}+(1-\omega) \rho^{k}, \\
\tilde{\rho}^{k+1} & =\nabla^{2} \rho^{k}, \\
\frac{\partial \rho^{k}}{\partial \mathbf{n}} & =0, \quad \text { at boundaries. }
\end{aligned}
$$

The parameter $\omega$ is called the over-relaxation parameter that speeds up the convergence of Gauss-Seidel method and is set to 1.7 or 1.8 .

The current density is obtained as the inverse of the gradient of the potential at each cell

$$
\mathbf{J}_{\mathrm{c}}=-\nabla \rho .
$$

Choosing the values of $\rho^{-}=0, \rho^{+}=d$ where $d$ is the linear distance between the contacts, we obtain a normalized value of current density, where nominal current density corresponds to the value $|\mathbf{J}|=1$. This current distribution is then used as a mask and scaled by the scalar value of the nominal current density applied in each simulation over a given sample, avoiding the computation of current distribution for each current value over the same shape.

As an example, in fig 4.3 the current distribution in an elliptical $1 \mu \mathrm{m}$ long platelet is computed, the current density direction is described by the arrows, while the intensity of current is shown in blue to red color scale.

\subsubsection{Effects of geometrical constriction on domain wall pinned dynamic}

An important step in the design of the device is the choice of a constriction that can maximize the precession window (4.24). An accurate study of this problem involves the use of a space dependent current density along the wire to reproduce the effect of the constriction on the electrons' flow. Several degrees of freedom appear at this point of our study: the constriction depth, width and shape, the width of the wire at the constriction and its nominal width. A selection needs to be made among the parameters to vary and this study is not intended to be exhaustive. As we will see later, numerous different situations can arise.

To explore the effect of geometrical constriction on the depinning threshold current, we perform a systematic study. We fix the dimension of the central channel to 


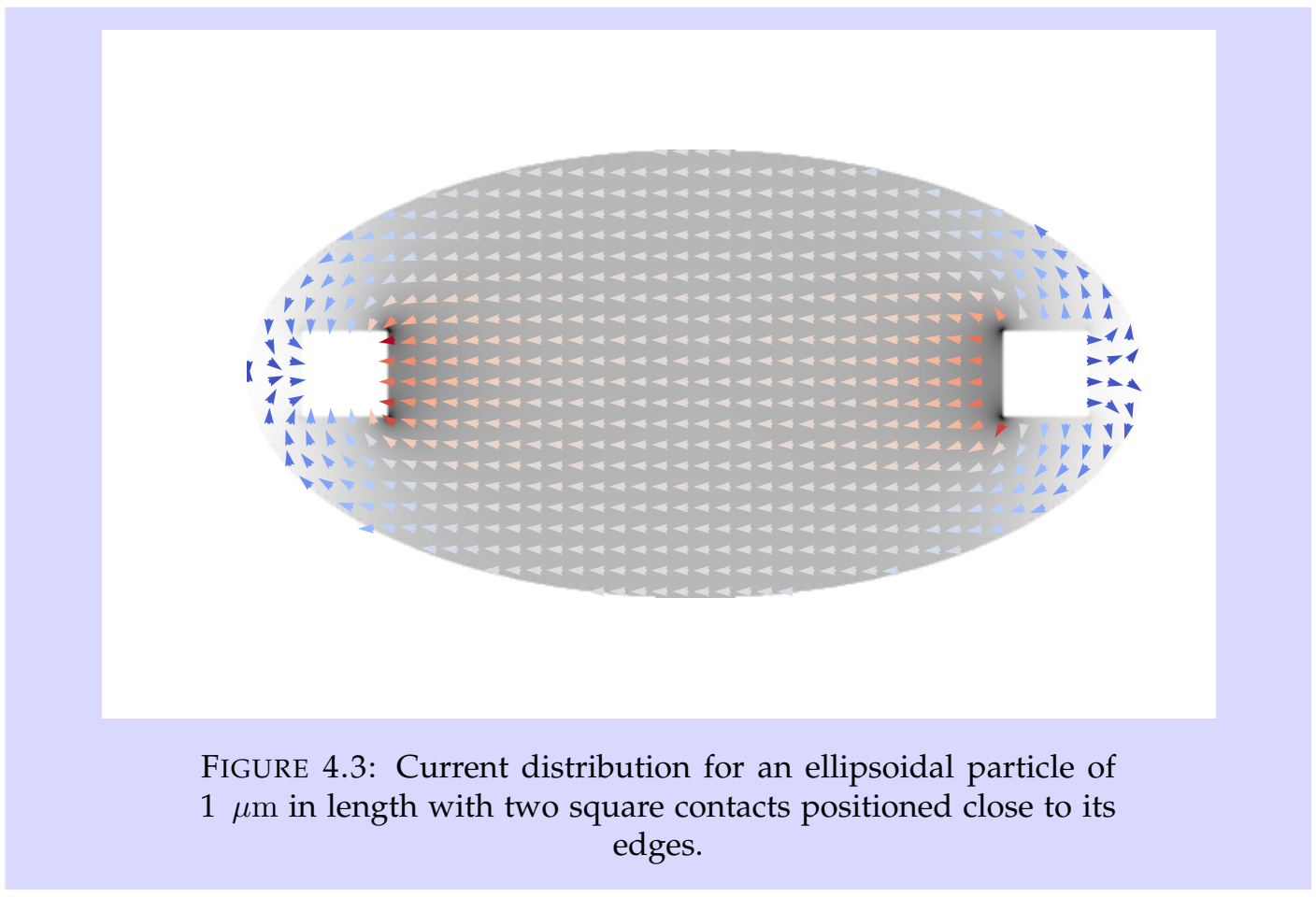

$20 \mathrm{~nm}$ and then vary the width of the ferromagnetic strip $L_{y}$ between 40 and $160 \mathrm{~nm}$; fixing the notch shape as symmetric triangular with three different slopes: $30^{\circ}, 45^{\circ}$ and $60^{\circ}$ as schematically presented in fig. 4.4. The thickness of the ferromagnet is fixed to $1 \mathrm{~nm}$ and material parameters of $\mathrm{CoFeB}$ are used for this study, with Gilbert's damping $\alpha=0.015$. In fig. 4.5, we show the current density distribution computed at three different geometrical constrictions along the wire. As expected, current flow increases at the constriction, where current density gradient is different for different constriction shapes.

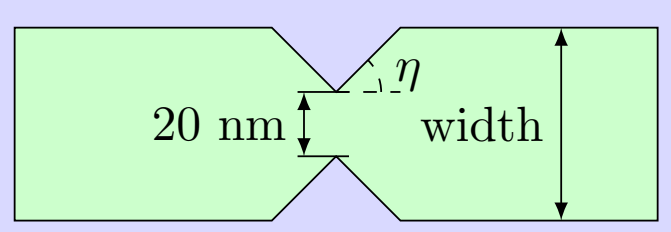

FIGURE 4.4: Geometrical specifications for depinning threshold study. The width at the centre of the notch is fixed to $20 \mathrm{~nm}$, while the width of the strip vary between 40 and $160 \mathrm{~nm}$. The slope of the notch takes three different values $\eta=30^{\circ}, 45^{\circ}, 60^{\circ}$.

The threshold current for depinning is found applying a given current density during 60 ns starting from a relaxed state with the DW pinned and looking at the DW velocity during the last $15 \mathrm{~ns}$. If the average velocity was larger than $0.05 \mathrm{~ms}^{-1}$ the DW was considered pinned. A bisection method algorithm was used, to reach an accuracy of $10^{8} \mathrm{Am}^{-2}$. 


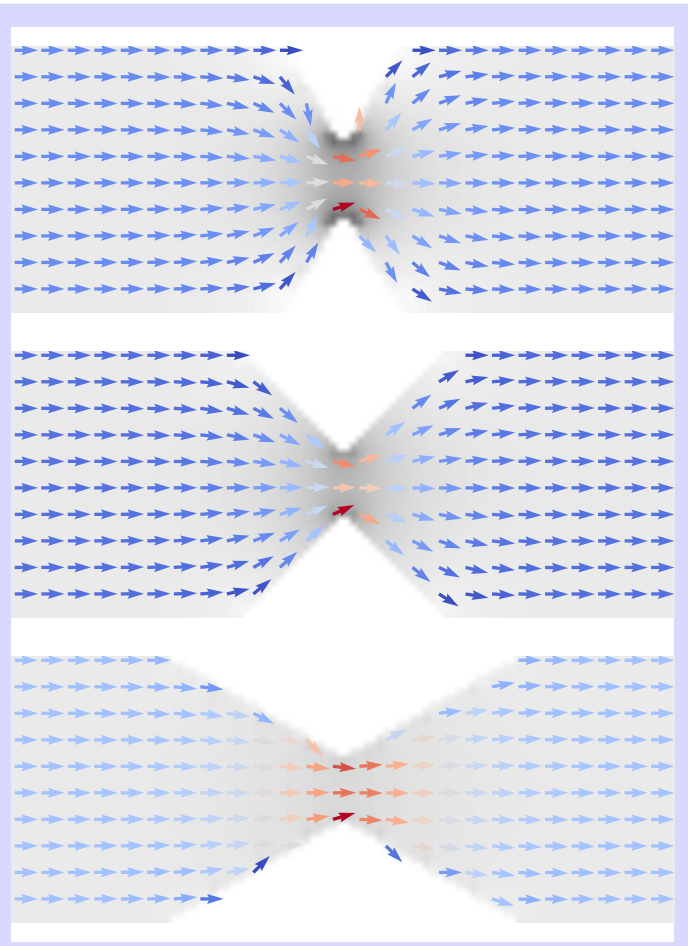

FIGURE 4.5: Current distribution for three different notch shapes, with edge slopes of 60,45 and 30 degrees from horizontal respectively. Contacts are placed at the endpoints of the wire, outside the figure. Arrows represent the direction of the electrons flow and the intensity is represented by the cold-hot color scale.

Figure 4.6-a,b show the depinning current density as function of wire width when measured along the strip cross section and at the notch, respectively. The plot showing the effective current density gives more information, since it takes into account the change in current density occurring at the notch due to the constriction and its variation with $L_{y}$. We can observe some particular features:

1 A plateau appears in fig. $4.6-\mathrm{b}$ for $L_{y} \geq 80 \mathrm{~nm}$ in the $30^{\circ}$ and $60^{\circ}$ cases. The steady position for the domain wall does not depend on the strip width any more: the confinement strength is equilibrated by the space-varying STT, whose strength decreases with distance from the channel. The general shape profile loses importance for wide wires and only the local values of current density and confinement-shape anisotropy need to balance. This happens always at the same distance from the centre of the notch (see fig 4.7-a,b). The nominal depinning current density on the other hand, needs to decrease with the width $L_{y}$ as $\propto L_{c} / L_{y}$, where $L_{c}$ is the channel width.

2 There is a jump in the critical current values for notch with slope of $45^{\circ}$. This is due to the existence of two possible points for a dynamical equilibrium between confinement and STT along the potential well created by the notch. 
Again, the reason for this effect lies in the space varying current density and space varying confinement force.

3 At the higher pinned-precession regime found for $45^{\circ}$ slope, the DW is no longer $1 \mathrm{D}$ and its precession is not uniform (see fig. $4.7-\mathrm{c}, \mathrm{d}$ ).

4 The initial increase of depinning current with strip width, more evident in the case of $60^{\circ}$ slope, is due to the ratio between decrease of current density away from the notch, which occurs more rapidly in the $60^{\circ}$ notch (see fig. 4.5), and also to the pinning potential strength, which is larger for the $60^{\circ}$ geometrical constriction.
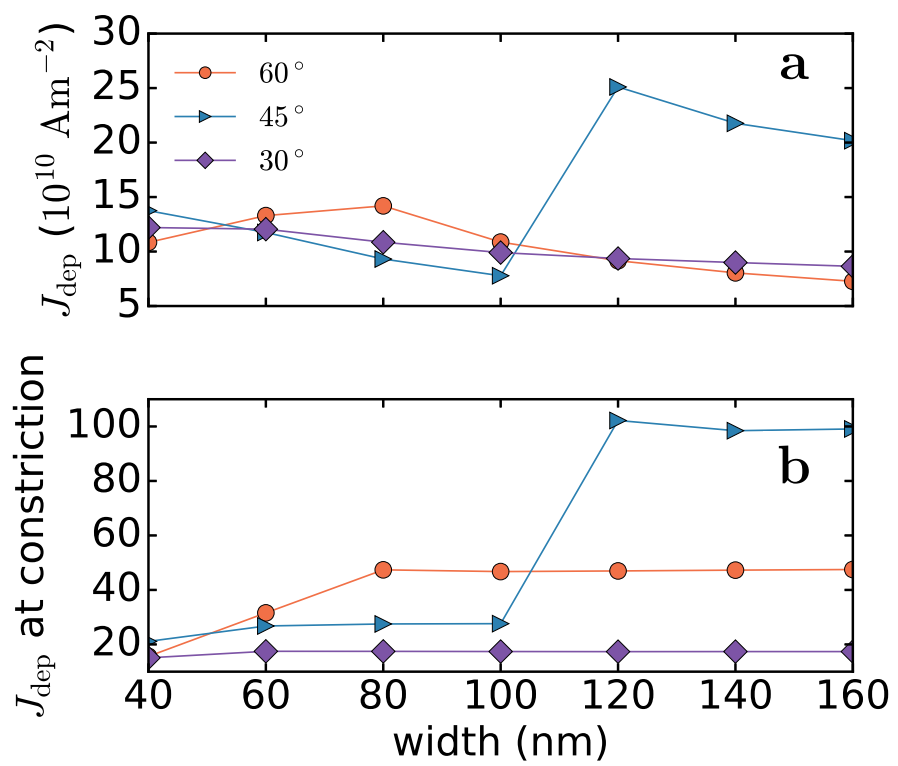

FIGURE 4.6: a. Nominal depinning current density (measured as flowing in the non-constricted strip) as function of strip width for three different notch slopes. b. Depinning current density (measured at the notch) as function of strip width for three different notch slopes.

For the study of spin wave emission from a pinned rotating domain wall we will select a wire that is $60 \mathrm{~nm}$ wide with a triangular notch with $45^{\circ}$ slope, resulting in a central channel $20 \mathrm{~nm}$ wide. This will assure us high depinning current and guarantee that the pinned DW precesses coherently over the whole range, while the computational effort for the spin waves propagation study will remain affordable. However, a wire of $20 \mathrm{~nm}$ in width will favour a Néel DW configuration and a quite strong shape anisotropy will have to be overcome to achieve DW rotation. 

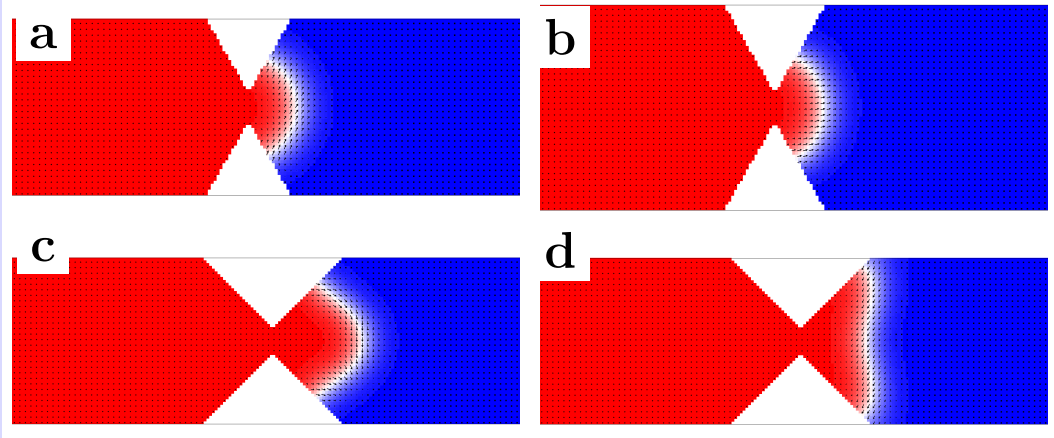

FIGURE 4.7: a.,b. Snapshots of magnetization at the limit current for precession with a notch of angle $60^{\circ}$ in wires of $120,140 \mathrm{~nm}$ wide respectively. The equilibrium position for DW precession is the same and does not depend on the wire width. c.,d. Snapshots of two different instant of time for DW pinned precession in a 120 nm wide strip with $45^{\circ}$ sloped notch. Here we are in the higher equilibrium point, the current is higher,and the DW precession is no longer coherent but presents internal modes and pronounced wobbling oscillation.

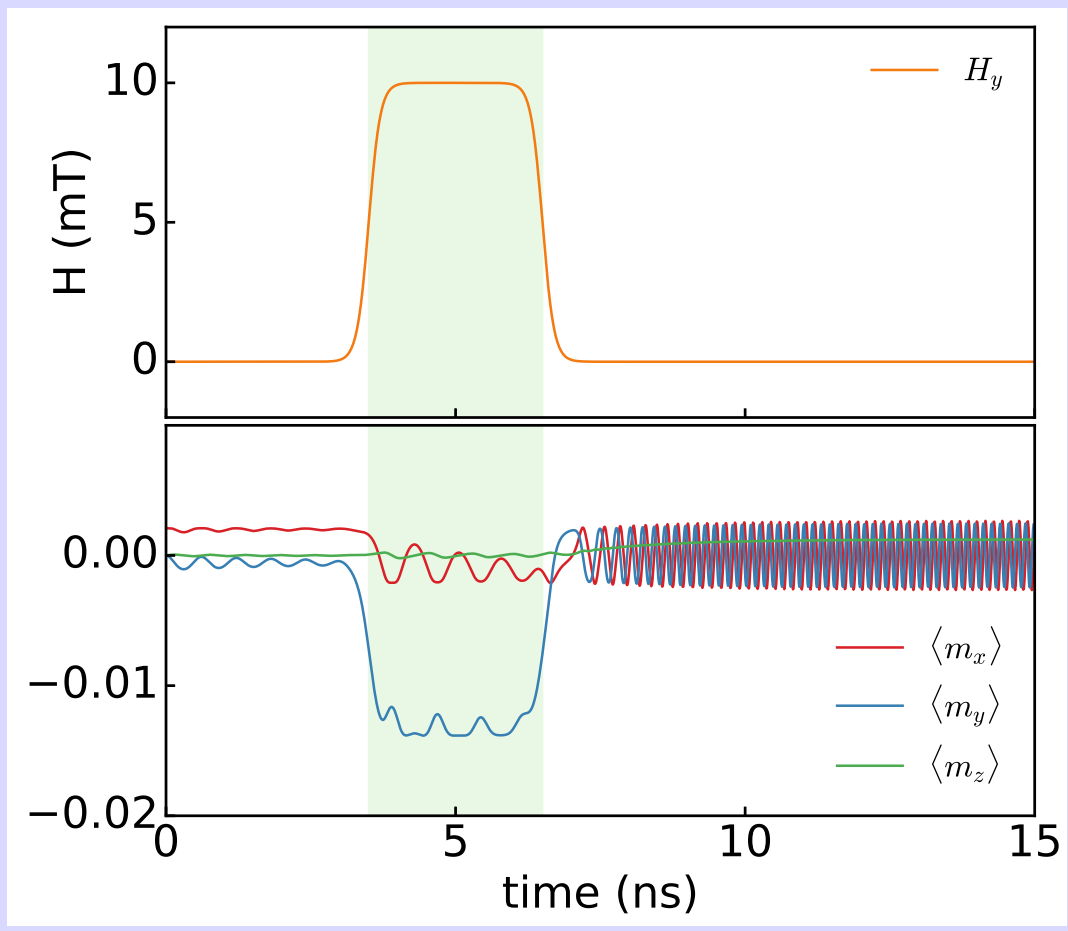

FIGURE 4.8: Application of an external field along the $-y$ direction to trigger domain wall pinned precession while a current density of $6.5 \times 10^{10} \mathrm{~A} \mathrm{~m}^{-2}$ is flowing along the wire.

\subsubsection{Out of equilibrium initial condition}

As it has been shown in section 4.2, the working window of the DW oscillator is bounded by the maximum pinning current at the top and by the shape anisotropy 
field at the bottom (4.24). Shape anisotropy torque opposes domain wall rotation, via the so-called intrinsic pinning in the purely adiabatic case $\beta=0$; however, in the presence of a pinning potential, we saw that shape anisotropy opposes initial rotation independently of the non-adiabatic coefficient. In particular, for a device with a central channel of $20 \mathrm{~nm}$, the domain wall minimum energy configuration is the Néel state and the threshold Walker current to induce precession from such initial state is high. However, the perturbation of this state via an external field pulse can overcome the intrinsic pinning and trigger the pinned precession state, as shown in fig. 4.8. Here, starting from an equilibrium Néel DW state, no rotation takes place upon application of an external current. The application of an external field pulse of $10 \mathrm{mT}$ along the $-y$-direction, is used to orient the DW so that the inplane shape anisotropy torque ( with $H_{K}$ positive if Néel configuration is favoured) is now concordant with the STT one (4.2). With this technique we can achieve DW pinned precession at very low current densities, overcoming the barrier of shape anisotropy.

In the following systematic micromagnetic simulations we will start with an equilibrium Bloch configuration, which is metastable due to symmetry of the system and equivalent to the situation of the system right after the pulse.

\subsection{Spin wave emission}

\subsubsection{Specifications}

In this study, we integrate numerically, the Landau-Lifschitz-Gilbert (LLG) equation of the magnetization dynamics (4.4) that includes the contribution of the spin transfer torque (4.1) caused by the flowing of an in-plane charge current density $\mathbf{J}_{c}$. Material parameters of annealed $1 \mathrm{~nm}$ thick $\mathrm{Co}_{20} \mathrm{Fe}_{60} \mathrm{~B}_{20}$ as in [52] were chosen: saturation magnetization $M_{s}=8.84 \times 10^{5} \mathrm{~A} \mathrm{~m}^{-1}$, uniaxial anisotropy constant $K_{u}=8.35 \times 10^{5} \mathrm{~J} \mathrm{~m}^{-3}$, exchange stiffness $A_{\mathrm{ex}}=23 \times 10^{-12} \mathrm{~J} \mathrm{~m}^{-1}$, Gilbert's damping $\alpha=0.015$. The degree of non-adiabaticity of the spin transfer torque was chosen as $\beta=2 \alpha$ and the polarization coefficient as $P=0.5$. The CoFeB strip under study is divided into square cells of $4 \mathrm{~nm}$ in side and $1 \mathrm{~nm}$ thick, with all dimensions below the Bloch length $\sqrt{A / k_{u}}=5.25 \mathrm{~nm}$. To avoid reflection of the SWs and simulate propagation in a much longer nanowire, absorbing boundary conditions are applied at the wire ends in the form of a smoothly augmented damping profile [124]. The spatial configuration of current density $\mathbf{J}_{c}$ is computed numerically by integrating Laplace's equation as discussed in 4.3.1. The LLG equation was integrated using a Runge-Kutta, Dormand-Prince predictor-corrector algorithm [38] with embedded error control presented in (2.1.6). Starting with a pinned Bloch DW configuration, simulations were run for $15 \mathrm{~ns}$ without saving output to skip the initial turbulent dynamics. Afterwards, simulations were run for 40 ns. The output was written every 5 ps. 


\subsubsection{Spin Wave Emission}

We consider a DW trapped at a symmetric constriction in a narrow wire and a dc current flowing through it, as shown in Fig. 4.9-a. The constriction acts as a pinning site for the DW and therefore, a minimum threshold current $J_{\text {dep }}(4.19)$ is required to depin the DW and propagate it through the nanowire. For current densities below this threshold, the DW remains pinned at a position where the restoring pinning force balances the driving STT force that pushes the DW away from the notch. However, a zero net driving force does not imply balance of the in-plane torques acting on the DW. In particular, if a current density is above a certain value $J_{\text {rot }}(4.24)$, the in-plane component of the STT overcomes the shape anisotropy field torque [125], leading to sustained full in-plane rotation of the spins inside the DW [117, 118].

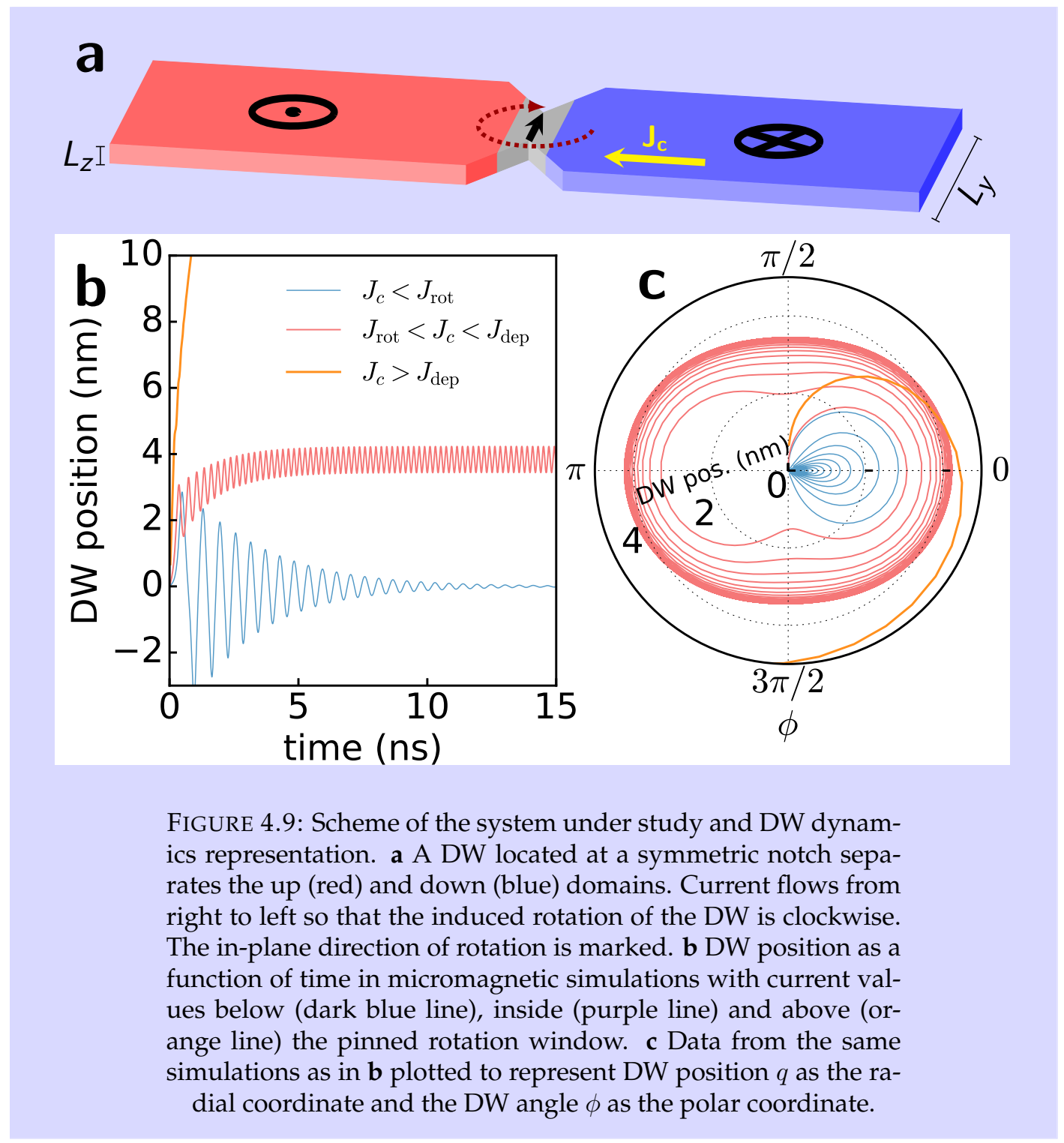

Data from micromagnetic simulations realized for three different cases, $J_{c, 1}<$ $J_{\text {rot }}<J_{c, 2}<J_{\text {dep }}<J_{c, 3}$ are shown in Fig. 4.9-b, where the DW position is plotted 
as a function of time, and in Fig. 4.9-c, where the position and in-plane DW angle are shown in polar coordinates. As can be observed, the DW reaches an equilibrium position for $J_{c}<J_{\text {rot }}$ after a few nanoseconds, whereas for $J_{c}>J_{\text {dep }}$, the DW rapidly depins from the notch. For $J_{\text {rot }}<J_{c}<J_{\text {dep }}$, however, the DW moves a few nanometres towards the right and slightly oscillates back and forth around this position while rotating in-plane.

To obtain a large operating window of the device, we tune the wire width and notch shape to obtain a low threshold current for the DW pinned rotation $J_{\text {rot }}$ and a high threshold current for DW depinning $J_{\text {dep }}$. Because the current density value is not uniform in space and increases at the constriction, throughout the chapter, we refer to its value as the nominal one away from the geometrical constriction. We select a wire width $L_{y}=60 \mathrm{~nm}$, thickness $L_{z}=1 \mathrm{~nm}$ and notch depth of $20 \mathrm{~nm}$, which gives us $J_{\text {rot }}=10^{10} \mathrm{~A} \mathrm{~m}^{-2}$ and $J_{\text {dep }}=12.75 \times 10^{10} \mathrm{~A} \mathrm{~m}^{-2}$, with the latter corresponding to a maximum current intensity of $7.6 \mu \mathrm{A}$ in the nanowire. The working window of such a device has the desirable quality of lying in a low-current density range, which allows us to avoid Joule heating effects and significant temperature gradients in proximity of the constriction [126].

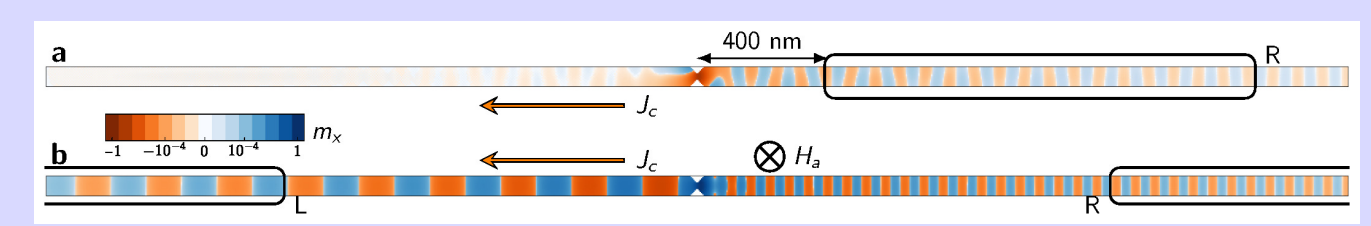

FIGURE 4.10: a. Snapshot of the magnetization dynamics representing $m_{x}=M_{x} / M_{s}$ value in color scale as indicated in the color bar. A current density of $6.5 \times 10^{10} \mathrm{~A} \mathrm{~m}^{-2}$ is injected. The region $R$ on the right where $m_{x}$ is sampled is enclosed by a rectangle. Unidirectional SW propagation towards the right can be observed. $\mathbf{b}$ Snapshot of the magnetization dynamics under the concurrent action of an in-plane current of $24 \times 10^{10} \mathrm{~A} \mathrm{~m}^{-2}$ and an external field of $300 \mathrm{mT}$ directed inside the plane to oppose DW depinning. A wire twice as long as in a is considered. Emission of SWs is observed both towards the right and the left at different frequencies and wavelengths; the sampled regions on the left $(L)$ and right $(R)$ are enclosed by black rectangles, and they extend $2 \mu \mathrm{m}$.

Upon the application of a current $J_{\text {rot }}<J_{c}<J_{\text {dep }}$ through the wire, the domain wall is driven towards the right and, after a transient time of a few ns (see Fig. 4.9-b), it reaches a stationary position below the notch, where it slightly oscillates back and forth (see Fig. 4.9-b) while its spins rotate clockwise in the strip plane, as shown in Fig. 4.9-c. Because of the reduced lateral dimension of the wire, DW rotation is coherent and its spins rotate synchronously. Examining the normalized $x$-component of magnetization $m_{x}=M_{x} / M_{s}$, as shown in Fig. 4.10-a for $J_{c}=6.5 \times 10^{10} \mathrm{~A} \mathrm{~m}^{-2}$, we observe the presence of the characteristic pattern of SWs propagating to the right side of the strip, whereas a much weaker propagation is observed to the left. 

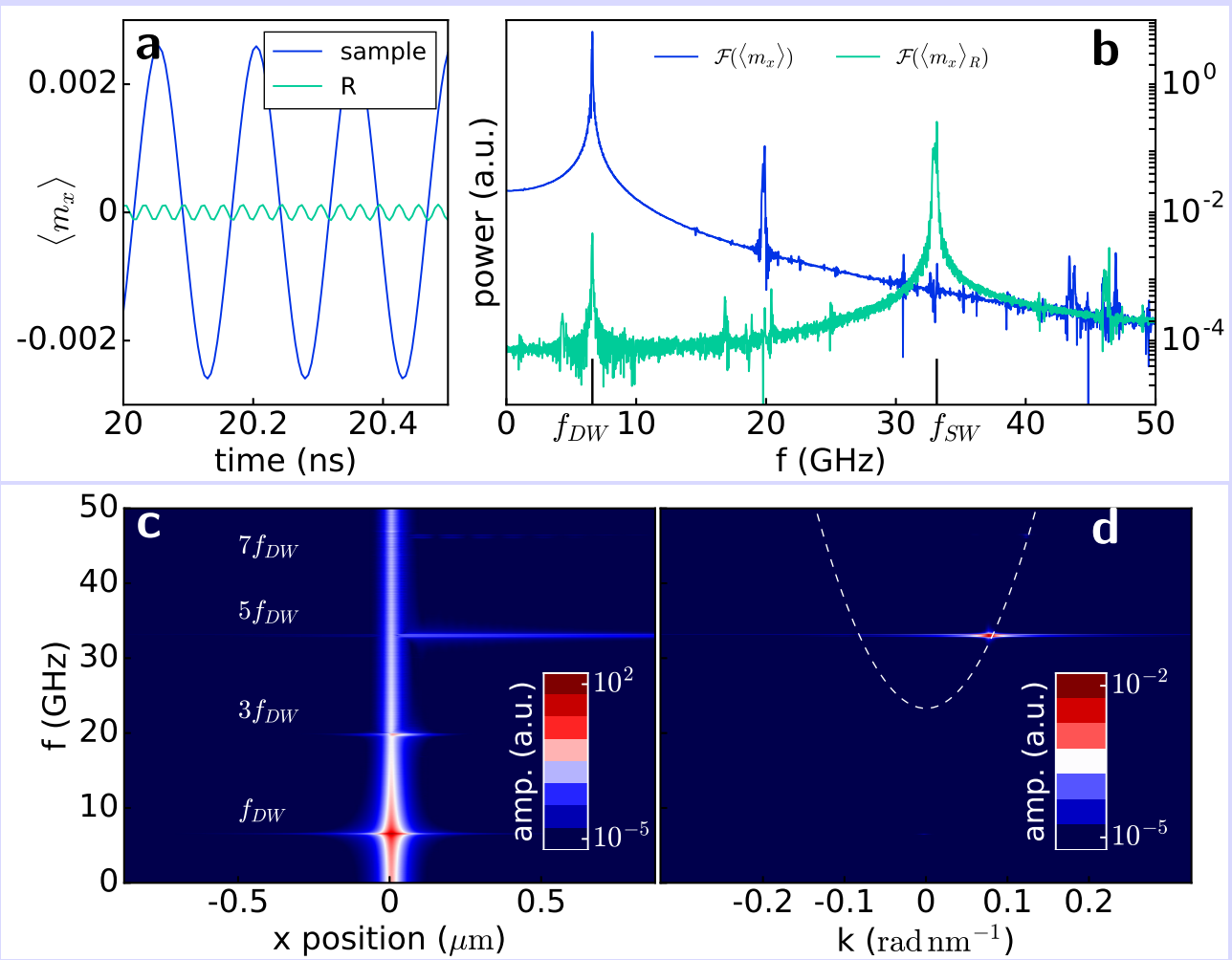

FIGURE 4.11: Study of DW rotation frequency and SW emission frequency. a Evolution of the averaged $x$ - component of the normalized magnetization during a time window of $0.5 \mathrm{~ns}$. The dark blue line shows averaging over the whole sample and the light blue line shows the averaging over the region $R$ away from the DW. Different periodicities can be observed. $\mathbf{b}$ Fourier transform of the time signals shown in a: the dark blue line shows the main peak at the frequency of rotation of the DW $f_{D W}$, and the light blue line has the principal peak at the SW propagation frequency $f_{S W}=5 f_{D W}$. c Frequency spectrum of $m_{x}(t)$ as a function of $x-$ position at the center of the strip width. Peaks centred at the DW position with an odd multiple frequency of $f_{D W}$ are marked.d $f-k$ diagram extracted from the central line in region $R$ shows a pronounced peak lying over the right principal branch of the analytical dispersion relation marked as a dashed line.

In Fig. 4.11-a we monitored the value of the $x$-component of magnetization averaged over the whole strip, $\left\langle m_{x}\right\rangle$ (dark blue line) and observed its value oscillate around zero at the frequency $f_{D W}=6.6 \mathrm{GHz}$. If we examine the average over a $1.35 \mu \mathrm{m}$ long region $R$, located $400 \mathrm{~nm}$ to the right of the DW (light green line), we observe a smaller oscillation at higher frequency. By taking the Fourier transform of these two signals (Fig. 4.11-b), we observe a peak at $f_{D W}=6.6 \mathrm{GHz}$ for the signal averaged over the whole strip, while the main signal from the region $R$ represents the SW frequency $f_{S W}=33.0 \mathrm{GHz}$. The secondary peak in the global signal, corresponding to $3 f_{D W}$, represents an odd higher harmonic. To gain more insight into the magnetization dynamics, we examine the frequency signal distribution over space 
(Fig. 4.11-c) by taking the Fourier transform of $m_{x}(t, \mathbf{r})$ at every cell situated along the $x$-central axis of the strip. A large amplitude signal can be observed at the centre of the strip where the DW rotates, remaining pinned below the notch, with the largest amplitude at the frequency $f_{D W}=6.6 \mathrm{GHz}$, at which the DW rotates fully in-plane. Additional peaks at odd multiples of $f_{D W}$ can be seen with a propagating branch corresponding to the fifth harmonic, indicating a definite propagation of the SW towards the right. Taking the Fourier transform in space and time of $m_{x}(t, \mathbf{r})$ in the same central row of cells restricted to region $R$, we obtain the $f-k$ diagram showing a single focused spot corresponding to $f_{S W}$ (Fig. 4.11-d). The analytical dispersion relation for exchange spin waves in our sample (4.42) is also shown in the figure:

$$
\omega(\mathbf{k})=\omega_{0}+\omega_{M} \lambda_{\text {ex }}^{2} \mathbf{k}^{2},
$$

where $\omega_{0}=\gamma_{0}\left(H_{\mathrm{k}, \mathrm{eff}}+H_{a}\right), H_{\mathrm{k}, \mathrm{eff}}$ is the effective out-of-plane anisotropy, $\omega_{M}=$ $\gamma_{0} M_{s}$ and $\lambda_{\text {ex }}^{2}=\frac{2 A}{\mu_{0} M_{s}^{2}}$. Indeed, $f_{S W}=5 f_{D W}=33.0 \mathrm{GHz}$ is the first odd harmonic that is allowed to propagate in the system, being above the threshold frequency $f_{0}=\omega_{0} / 2 \pi=23.3 \mathrm{GHz}$. This emission of SWs has the remarkable property of being unidirectional, coherent and directly dependent on the applied current density as we will discuss below.

Varying the applied current intensity between $J_{\text {rot }}$ and $J_{\text {dep }}$ leads to different DW rotation frequencies extracted from $\mathcal{F}\left(\left\langle m_{x}\right\rangle\right)$, as shown in Fig. 4.12-a and b. In addition, the amplitude of the emitted signal increases with current density, as denoted by the size of the hexagons in Fig. 4.12-b. This observation is due to the fact that $\left\langle m_{x}\right\rangle$ oscillation increases with $J_{c}$ because the DW moves further away from the centre of the notch and, therefore, its length increases and so does the number of spins precessing.

It is predicted by the analytical model [116] that a linear relationship exists between $f_{D W}$ and the applied current (4.20). However, the spatial variation of current density introduces additional complexity to the problem. In fact, local current density at the DW is maximum when it is located at the centre of the notch and it decreases as the DW is pushed away from the centre, as shown in Fig. 4.5. This is clarified by looking at both DW rotation frequency and average position as function of the current density at the DW position as it is shown in Fig. 4.13-b. As the current density increases, the DW moves further away from the notch where current density is lower. As can be observed, if we take the data from Fig. 4.13-a (same as Fig.4.12-b) and plot them against the current $J_{c}$ flowing at the DW position, we first obtain a linear increase of $f_{D W}$ with $J_{c}$, as predicted by the analytical model (4.20). Above $8.5 \times 10^{10} \mathrm{~A} \mathrm{~m}^{-2}$, however, the further displacement of the DW from the centre of the notch and concurrent reduction of the local current density yields a stabilization of the effective current density flowing at the DW, yielding an almost constant $f_{D W}$. Average DW displacement is computed from micromagnetic simulations as $\Delta x_{D W}=-\frac{L_{c}}{2}+\sqrt{\frac{L_{c}^{2}}{4}+A_{t o t}\left\langle m_{z}\right\rangle}$, representing the width of the trapezoidal region 

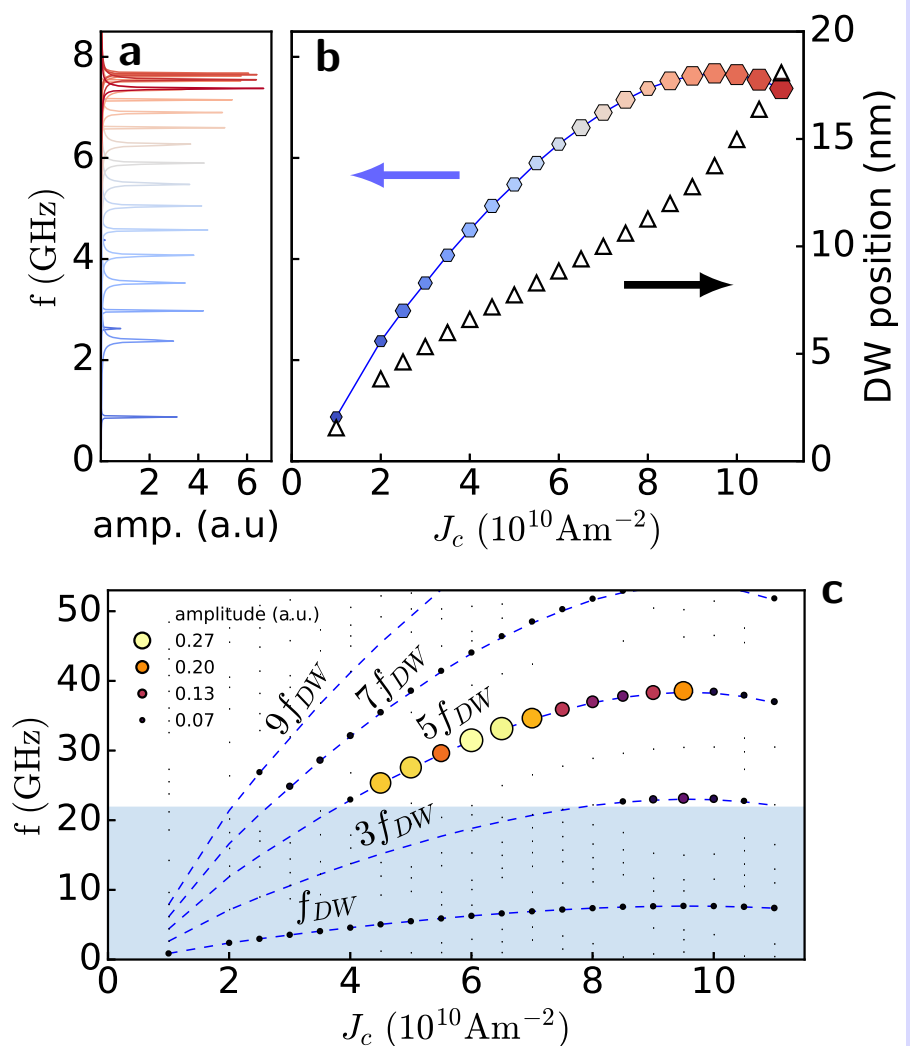

FIGURE 4.12: Frequency analysis of DW rotation and SW emission. a Frequency spectra of $\left\langle m_{x}\right\rangle$ showing the peaks from which $f_{D W}$ is extracted. b DW frequency (full hexagons) as a function of applied current extracted from the peaks in a; the size of the hexagons is proportional to the height of the peaks. Average DW position in the pinned rotation regime for the corresponding current (triangles). c Principal peaks in the frequency spectrum extracted from region $R$ away from the DW. The peak height is denoted by the circle size and the colour scale, (dark to bright). The dashed lines denote $f_{D W}$ as in $\mathbf{b}$ and its odd multiples. The shaded region denotes the non-propagating frequency gap $f<f_{0}$.

that reversed magnetization, assuming the DW as a straight line, as schematically shown in inset in Fig. 4.13-a, $A_{t o t}$ being the total upper surface of our sample.

If we now examine the frequency spectrum away from the notch, we observe a different distribution of amplitude peaks with $J_{c}$. In Fig. 4.12-c, peaks in the frequency signal sampled over region $R$ are plotted against the applied current density with different colours and sizes to mark their amplitude. Dashed blue lines denote the frequency-current density curve of the rotating DW and its odd higher harmonics. As can be observed, all peaks lie on odd harmonics of $f_{D W}$, and their amplitude is maximum when SW can actually propagate, i.e., above the threshold frequency $f_{0}$. The frequency gap region where propagation is forbidden is shaded 


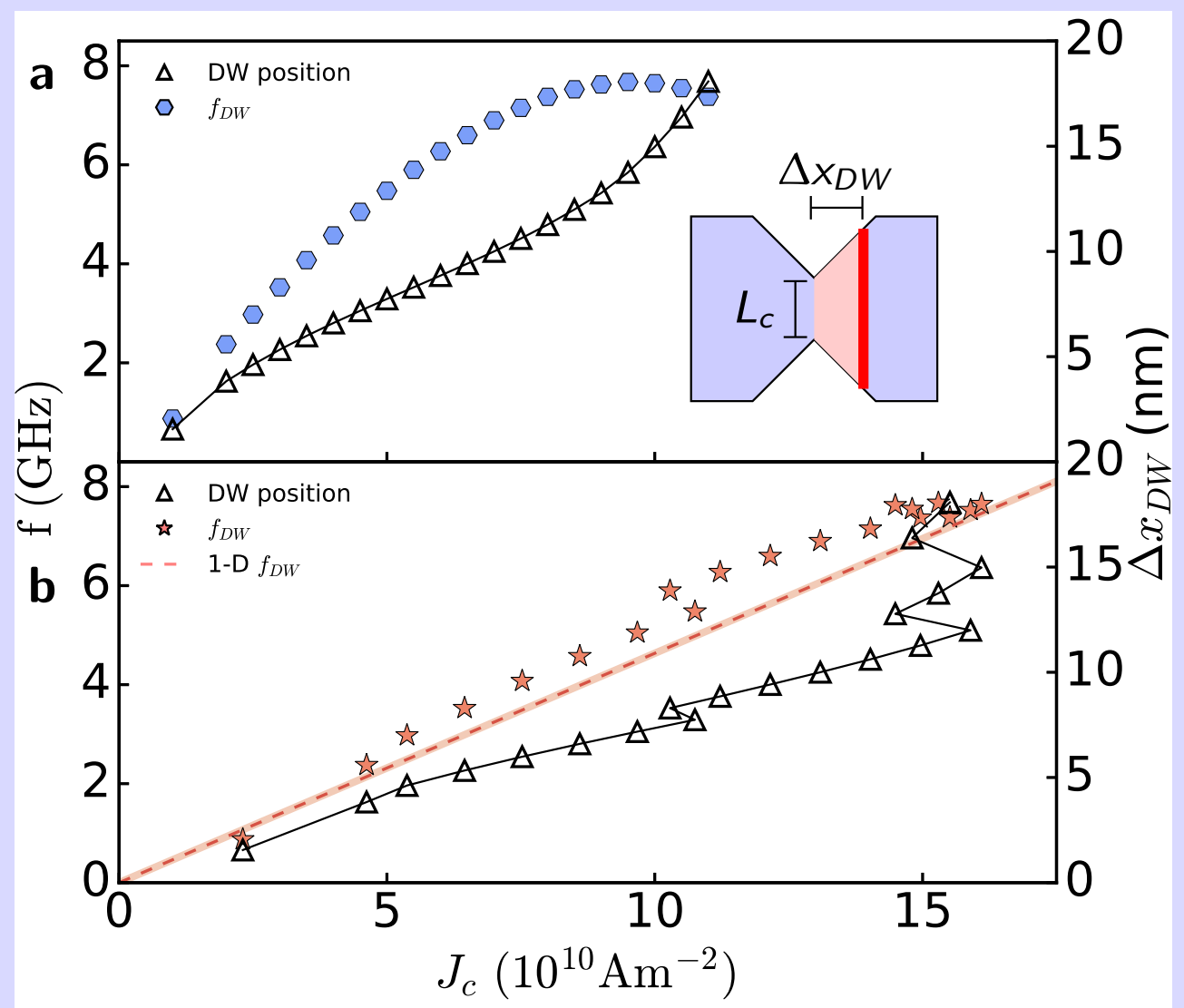

FIGURE 4.13: a DW rotation frequency and position as function of nominal current density. Inset: schematic showing how $\Delta x_{D W}$ is evaluated. b DW rotation frequency and position as function of the current density measured at the centre of the DW.

Dashed line is the analytical prediction from equation (4.20).

in blue. The highest emission intensity is achieved for current densities between 4.5 and $7 \times 10^{10} \mathrm{~A} \mathrm{~m}^{-2}$ on the fifth harmonic, which is the first branch largely above the propagation threshold $f_{0}$. Emission is highly coherent with linewidths below 150 MHz.

\subsubsection{Application of external field}

To extend the operating window of the device, we apply an external field opposing the driving force exerted by the STT. In our situation this means applying an external field $H_{a}$ pointing into the plane along the $-\hat{\mathbf{z}}$ direction. We can estimate such an effect by means of the 1D model: the external field together with the pinning effective field must balance the STT so that we have a linear dependence of the depinning current on the external field:

$$
J_{\mathrm{dep}}\left(H_{a}\right)=\frac{e M_{s}}{P \mu_{B}} \frac{\alpha \gamma_{0} \Delta}{1+\alpha \beta}\left(H_{p}(\bar{q})+H_{a}\right)=J_{\mathrm{dep}}^{0}+\frac{e M_{s}}{P \mu_{B}} \frac{\alpha \gamma_{0} \Delta}{1+\alpha \beta} H_{a},
$$


where $J_{\text {dep }}^{0}$ is the threshold current for depinning without an applied field from equation (4.24).

The increase in the depinning current in the presence of an external field is shown in Fig. 4.14-a together with the analytical prediction (4.50) (dashed line). Threshold current $J_{\text {dep }}$ increases almost linearly with the applied field for a wide range of fields, with $120 \mathrm{mT}$ giving a $100 \%$ increment of the depinning current at zero field. In this manner, we can extend the current density window for DW rotation and achieve higher DW rotation frequencies (up to $15.7 \mathrm{GHz}$ at $J_{c}=22 \mathrm{~A} \mathrm{~m}^{-} 2$ for an applied field of $300 \mathrm{mT}$ ) as shown in Fig. 4.14-c. An interesting consequence of the application of the field is its antisymmetric contribution to the effective field in the two magnetic domains in which our strip is divided, which leads to the vertical displacement of the left and right propagating branches (Fig. 4.14-b), depending on the relative orientation with the magnetization.

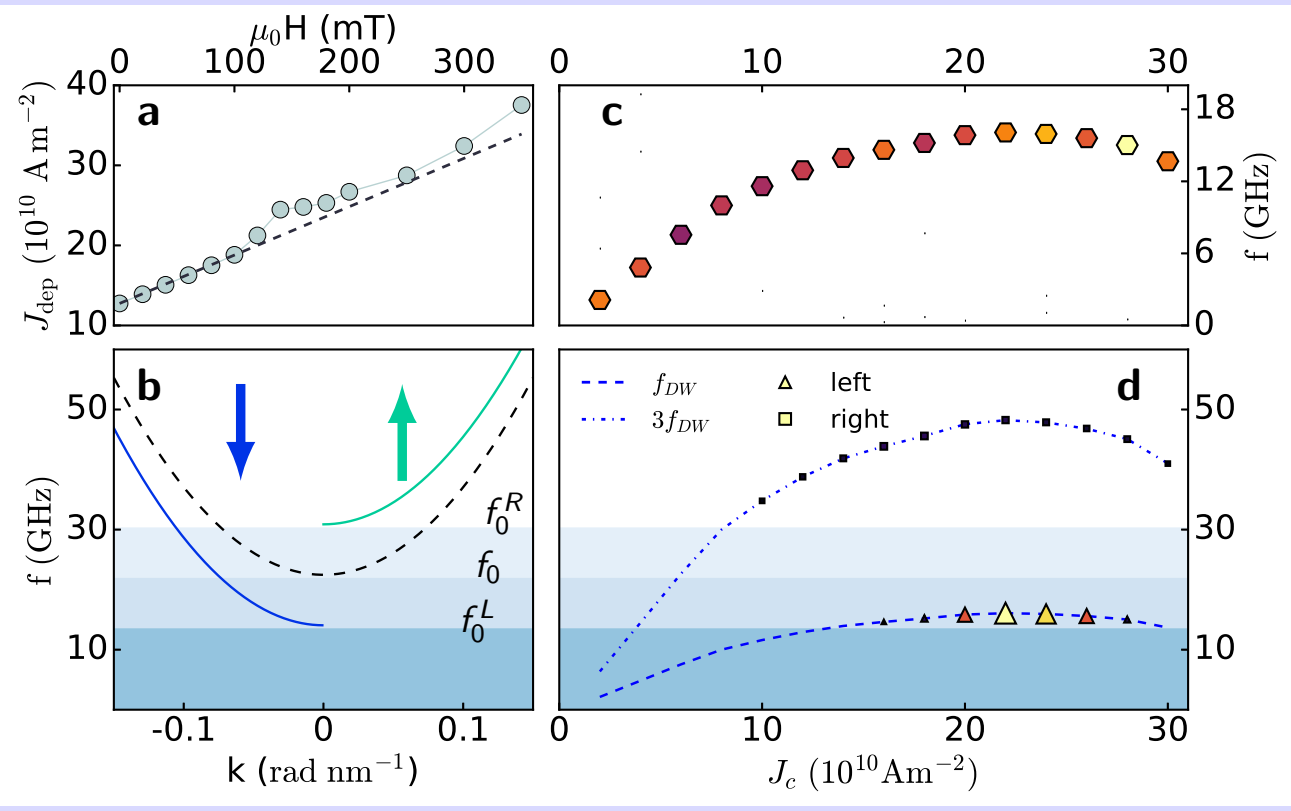

FIGURE 4.14: DW rotation and SWs dynamics in the presence of an external field. a Depinning current $J_{\text {dep }}$ as a function of a counter-acting external field applied along the $-\hat{\mathbf{z}}$ direction. The dashed line represents the theoretical prediction made using the one-dimensional model (4.50). b Splitting of the dispersion relation branches in the positive and negative directions with respect to the DW caused by the application of an external field, as prescribed by equation (4.49). c DW rotation frequency as a function of the applied current when an external field of $300 \mathrm{mT}$ is applied. $\mathbf{d}$ SW propagation observed in the regions distant from the DW on the left (triangles) and on the right (squares), as indicated in Fig. 4.10-b. The size of the symbols express the $\mathrm{SW}$ amplitude. The SW propagates to the left at $f_{D W}$, whereas it propagate to the right at $3 f_{D W}$. 
possibility of SW propagation in the left domain. In fact, for an applied field of $300 \mathrm{mT}, f_{0}=23.3 \mathrm{GHz}$ becomes $f_{0}^{R}=31.4 \mathrm{GHz}$ and $f_{0}^{L}=14.6 \mathrm{GHz}$ for the right and left domains, respectively, with the latter being below $f_{D W}$ for a wide range of $J_{c}$ (see Fig. 4.14-c and d). We perform simulations with an external applied field $-H_{a} \hat{\mathbf{z}}$ with $\mu_{0} H_{a}=300 \mathrm{mT}$ and we monitor the magnetization in two regions $L$ and $R$, both of them $2 \mu \mathrm{m}$ long and situated $1.296 \mu \mathrm{m}$ from the centre of the strip, as shown in Fig. 4.10-b. The DW rotation frequency is extracted as usual as $\mathcal{F}\left(\left\langle m_{x}\right\rangle\right)$ and is plotted in Fig. 4.14-c. In Fig. 4.14-d, the peaks in frequency of $m_{x}(t)$ in the two sampled regions are plotted, where the size of each symbol represents the peak's amplitude. In the left domain (triangles), we have SW propagation towards the left at the DW rotation frequency when this exceeds the propagation threshold frequency $f_{0}^{L}$, and no higher harmonic excitation is observed. On the right side, the third harmonic is now accessible for SW propagation via the increased DW rotation frequency and is the one at which SWs propagate. This result adds an important feature to this spin wave emitter because spin wave propagation can be tuned in two different aspects: the propagation frequency can be regulated by changing the applied current, while bidirectional or unidirectional emission from the DW and additional frequency regulation can be selectively chosen via the application of an external field.

\subsubsection{Role of dipolar field and unidirectionality}

This novel scheme for tuneable and short-wavelength SW emission suggests new directions in low-power magnonic devices. Unidirectional and asymmetric spin wave propagation is a peculiar feature of this system. The intrinsically asymmetric nature of the Dzyaloshinskii-Moriya interaction (DMI) has been exploited to obtain unidirectional propagation of SWs along nanowires [127] and focusing of SWs in thin films [128]. However, in our system, the effect of DMI is negligible, and the origin of such an effect is purely geometric, as will be shown below. The precession of the domain wall's spins is the source of spin wave excitation, and SW propagation at odd higher harmonic of the DW rotation frequency is the signature of a periodic and non-linear excitation [129, 113]. If the simple oscillation of the DW below the notch or its change in width when passing from Neel to Bloch configuration were the main mechanism of excitation, we would observe emission at $2 f_{D W}$ and its harmonics. However, the absence in the frequency spectrum of the amplitude peaks at even multiples of $f_{D W}$ makes us disregard this hypothesis.

To shed more light on the excitation mechanism, we focus our attention on the role played by the stray field of the rotating DW. Because of the reduced width of the strip, precession of the spins in the DW occurs in a very coherent fashion, making the DW appear as a dipole rotating in the strip plane, as represented schematically in Fig. 4.15-a. The stray field generated by such a dipole has a strong in-plane component and it rotates at $f=f_{D W}$. To verify that the DW behaves as a rotating dipole and to show that this is the main mechanism that excites propagating SWs, we proceed in two steps. First, we examine the SW emission induced in a 
uniformly magnetized thin film via the external field generated by a point dipole $\mathbf{B}(\mathbf{r})=\frac{\mu_{0}}{4 \pi}\left(\frac{3 \mathbf{r}(\mathbf{m} \cdot \mathbf{r})}{r^{5}}-\frac{\mathbf{m}}{r^{3}}\right)$ rotating in-plane at $f=5 \mathrm{GHz}$. After a period of transient turbulent dynamics with incoherent emission of SWs, when a stationary regime is reached, we observe an isotropic and rather weak emission of spin waves in all directions (Fig.4.15-b). When 2-dimensional Fourier transform is performed on $m_{x}(t)$ along a $1.25 \mu \mathrm{m}$ long line starting $400 \mathrm{~nm}$ from the centre of the square (rectangular region in fig. 4.15-b), we see in the $f-k$ diagram a spot at $5 \mathrm{GHz}$ and $k=0$ corresponding to the non-propagating oscillation induced directly by the external dipolar field. The principal branch of the propagating SW is also marked, showing a peak at $25 \mathrm{GHz}$; i.e., emission is stronger at a frequency 5 times larger than the driving rotation rate. In other words, the rotating dipolar field is responsible for excitation of SWs at odd harmonics and such excitation is weak, comparable to the one also observed in the left domain in Fig. 4.10-a.

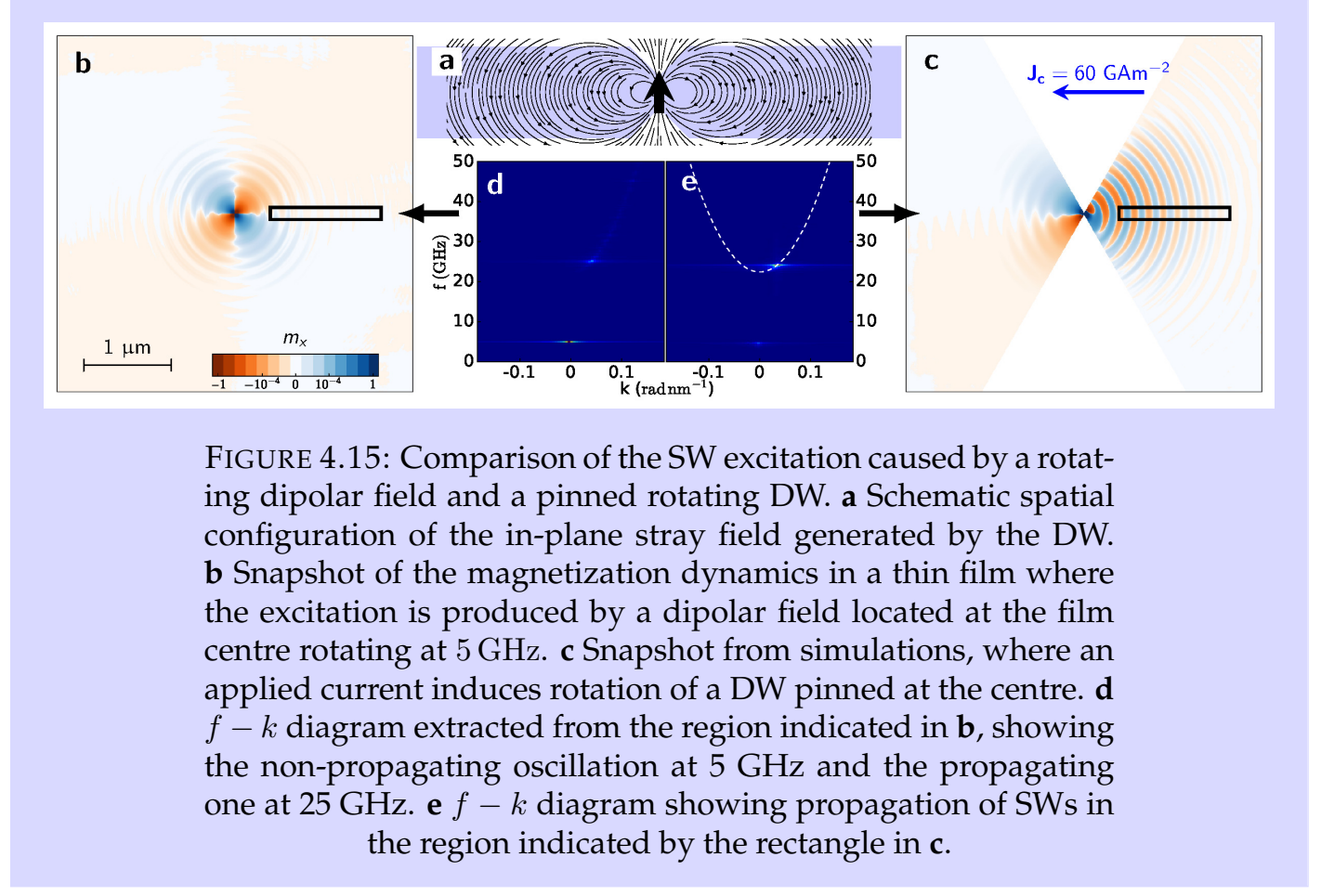

In order to highlight the analogy with the case of the rotating DW, we carve a very deep symmetric notch in the square film to obtain a $20-\mathrm{nm}$ channel in the middle, as in the nano wires under examination. We then set an up-down magnetization configuration with the DW pinned at the channel and apply a current of $6 \mathrm{GA} \mathrm{m}^{-2}$ (uniform for simplicity) that yields a DW rotation frequency $f_{D W} \sim 4.8 \mathrm{GHz}$. In Fig. 4.15-c, a snapshot representing $m_{x}$ during the stationary dynamics shows a strong SW emission to the right, whereas the perturbation in the left domain is much weaker and is not capable of exciting SWs. Extracting the $f-k$ diagram from the same spatial region and over the same time span as in the rotating dipole case (Fig. 4.15-e), we find a spot at $f_{D W}$ and $k=0$, while the spot on the dispersion 
relation branch is exactly at $24 \mathrm{GHz}=5 f_{D W}$ with no additional SW emission along the branch.

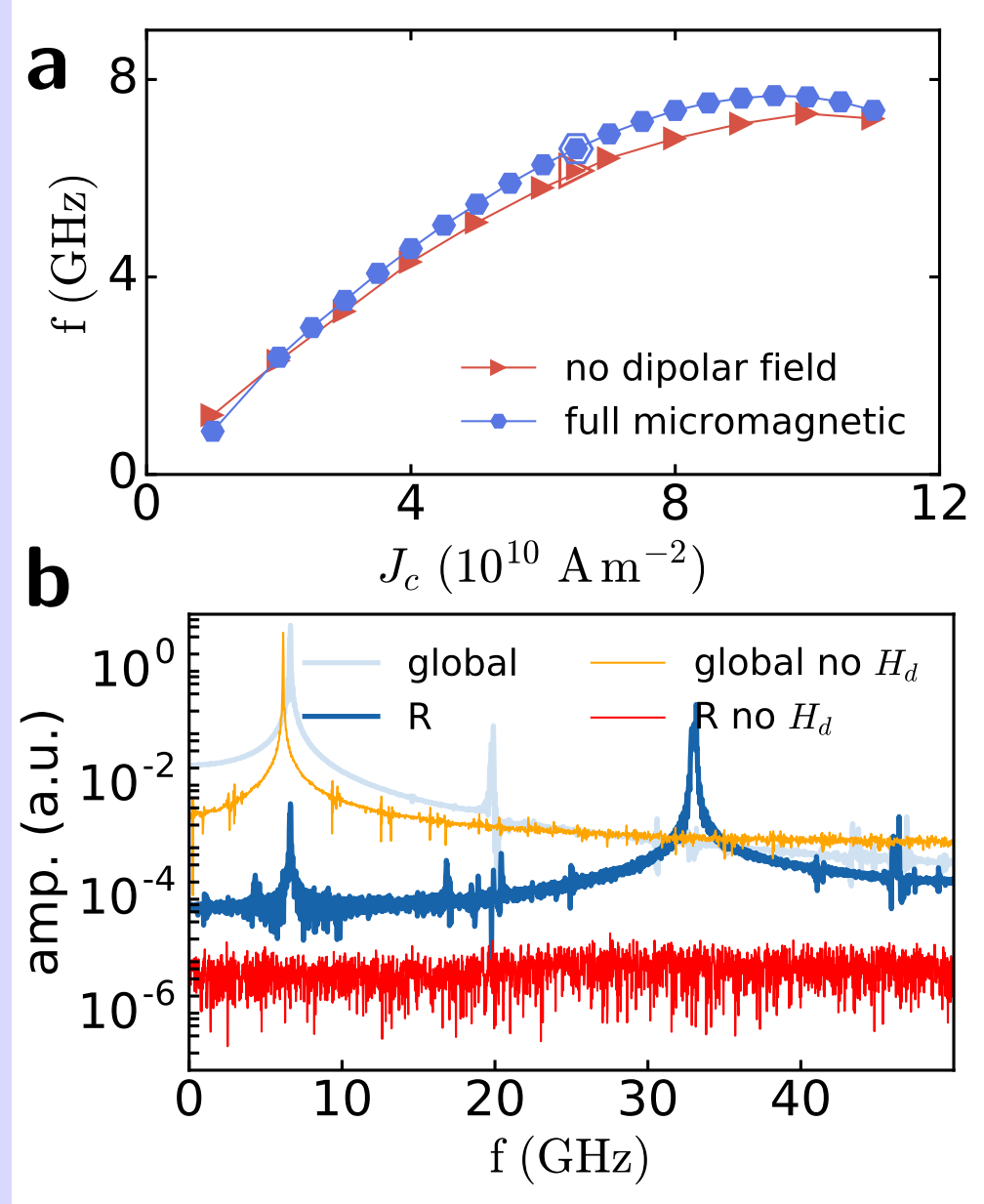

FIGURE 4.16: Role of non-local stray field in exciting SWs. a DW rotation frequency as a function of current for full micromagnetic simulations (blue hexagons) compared with simulations without the non-local effect of the magnetostatic field (orange triangles). b Fourier transform of $m_{x}(t)$ averaged over the whole sample and in the region $R 400 \mathrm{~nm}$ from the notch, as in Fig. 4.10-a (light and dark blue lines), under the application of $6.5 \times 10^{10} \mathrm{~A} \mathrm{~m}^{-2}$. Simulations without computation of the dipolar fields (orange and red lines) show a single peak at the DW frequency and no signal away from the DW.

To further prove the essential role of the DW's stray field in exciting SWs, we run micromagnetic simulations without considering the long-range dipolar interaction; i.e., considering an anisotropy parameter $K_{\text {eff }}=K_{u}-\frac{1}{2} \mu_{0} M_{s}^{2}$ that includes the local demagnetizing effect of the dipolar field, we can achieve DW pinned rotation with a behaviour and a rotation frequency very similar to those observed in full simulations (Fig. 4.16-a). However, only a weak dissipative radiation of exchange SWs is present $[112,114]$, and no coherent SW emission at a well defined 
frequency is observed, as shown in Fig. 4.16-b, where the frequency spectrum at $J_{c}=6.5 \times 10^{10} \mathrm{~A} \mathrm{~m}^{-2}$ is compared with the standard simulations that take into account dipolar interaction. From these observations, we can conclude that the dipolar field of the DW is responsible for the coherent excitation of SWs, behaving as a rotating antenna. However, additional contributions from the wire edges must be considered to explain the unidirectionality. When neglecting dipolar interaction, the DW rotation excites circular oscillations in the spins close to the DW only via exchange interaction passing from Bloch to Néel configuration at $f_{D W}$ frequency. Such perturbation is very strong close to the DW and decays exponentially with distance. On the other hand, the magnitude of the DW dipolar field decays as $|\mathbf{r}|^{-3}$ from the DW. In Fig. 4.17-a and b, the magnitude of the in-plane component of both exchange $\left(H_{e x}^{\mathrm{ip}}\right)$ and dipolar $\left(H_{d}^{\mathrm{ip}}\right)$ fields is shown in dark to bright colour scale before applying the external current, when the DW is pinned at the centre of the notch pointing upwards and no propagating SW perturbs the configuration. Moreover, when the DW is set into rotation by the application of a current, the in-plane component of the two fields rotates in opposite directions: clockwise $H_{e x}^{\mathrm{ip}}$ following the DW rotation and anticlockwise $H_{d}^{\mathrm{ip}}$, so there is competition between the excitation of the spins due to the exchange interaction close to the DW and that due to dipolar interaction further away from the DW. Their combined effect results in a strongly elliptical excitation of the magnetization in the region where the two fields have a similar magnitude, as shown in Fig. $4.17-\mathrm{c}$, which produces the non-linear excitation of SW and the generation of higher harmonics. Furthermore, the fact that such excitation is periodic, leads to the cancellation of the excited modes of even order, allowing propagation of the odd higher modes exclusively.

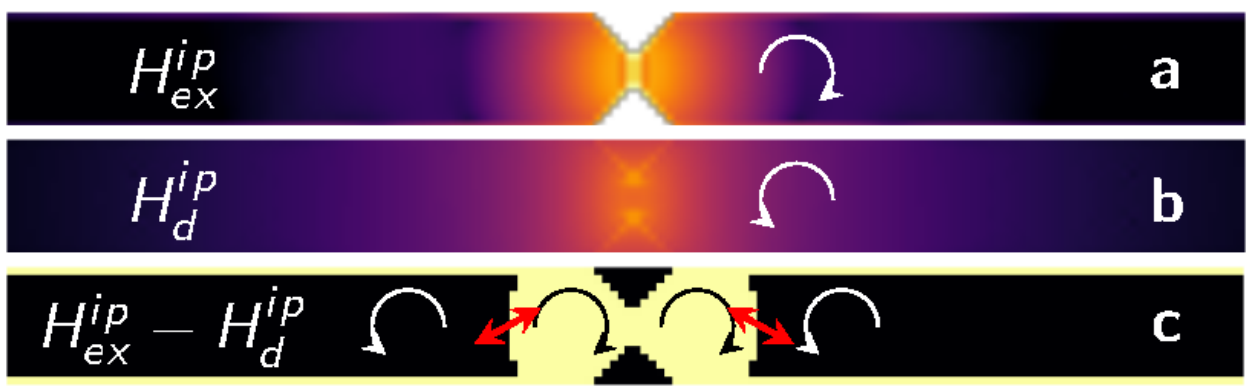

FIGURE 4.17: Competition between the in-plane components of exchange and dipolar field is the origin of SW excitation. The intensity of the in-plane component of the exchange field (a) and the dipolar field (b) for a DW placed at the center of the wire at rest is plotted in dark to bright colour scale. The arrow indicates the direction of rotation of the in-plane component of the field when the DW is led to rotation via an applied current. c The sign of $H_{e x}^{i p}-H_{d}^{i p}$ marked as bright (dark) for positive (negative). Arrows indicate the direction of rotation of the combined in-plane excitation. 


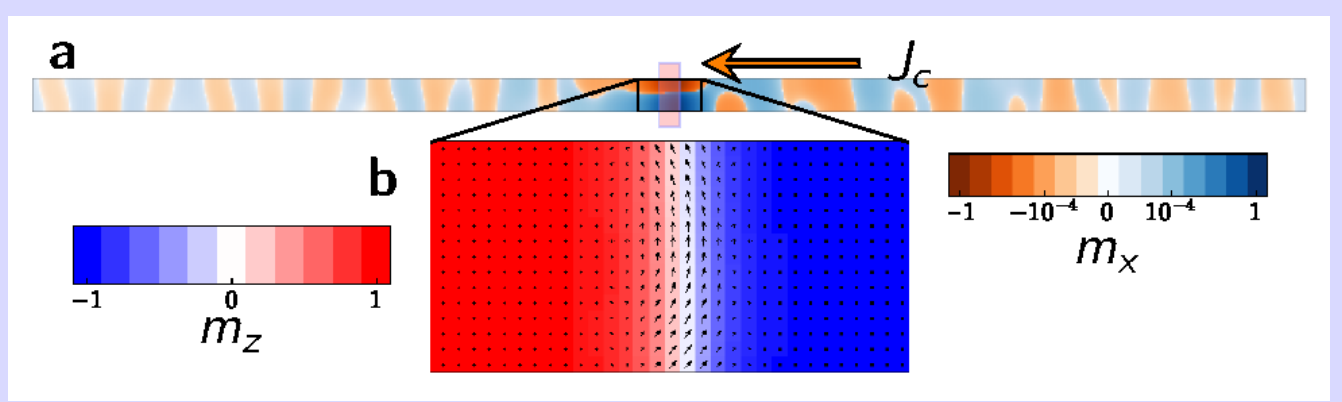

FIGURE 4.18: Non-geometrical constriction produces bidirectional SW emission. a Snapshot of the magnetization dynamics showing the $x$ - component of the magnetization when a DW is forced to rotate via the application of a current density $J_{c}=9 \times 10^{10} \mathrm{~A} \mathrm{~m}^{-2}$. The pinning is realized via a decrease of $20 \%$ in uniaxial anisotropy constant $K_{u}$ in the shaded region. b Close view of DW magnetization distribution. The DW stretches across the whole wire width and its rotation is no longer uniform, causing a more irregular SW excitation and waveform.

At the wire edges, where in-plane tilt of the spins produces surface charges and an additional stray field component, this effect is strengthened. The fact that the DW is pushed by the STT from the centre of the notch towards the right makes the excitations at the edges much weaker on the left side, where both fields have small in-plane components, so that their combined action is not capable of exciting higher harmonics. To prove this point, simulations with a different DW pinning strategy have been performed. A $20 \%$ lower uniaxial anisotropy constant $K_{u}$ in a 30-nm wide band at the centre of the nanowire (indicated by the blue rectangle in Fig. 4.18a) creates an energetically favourable position for the DW, giving rise to a strong and localized potential well for the DW without changing the local geometry. Applying a current $J_{c}=9 \times 10^{10} \mathrm{~A} \mathrm{~m}^{-2}$ produces SW emission in both directions (as shown in Fig. 4.18-a). This result also proves that the small non-adiabatic torque we use does not play a role in suppressing spin waves that propagate against electron flow, as it has been argued in the literature [130]. Therefore, we conclude that the dipolar field of the rotating DW is responsible for the higher harmonic SW emission, and because this excitation has a geometrical dipolar origin, the displacement of the DW on the right side of the pinning site causes the screening of the emission towards the left side. Such emission is recovered when $f_{D W}>f_{0}$, and the simple DW rotation can excite the SW towards the left (see Fig. 4.10-b and 4.15-d).

In conclusion, we have presented a novel paradigm to excite spin waves via the spin transfer torque-induced rotation of a domain wall pinned at a geometrical constriction in a narrow wire. We showed that, by selecting the notch shape and the wire cross section, spin wave emission in the direction of electrons' flow can be achieved for extremely low current densities. Spin wave emission occurs at an odd multiple of the DW rotation frequency up to $40 \mathrm{GHz}$, without any external applied field. Such 
spin wave emission is very coherent and of short wavelength down to $60 \mathrm{~nm}$, typical of exchange spin waves. The application of an external field opposing the STT has the twofold effect of, on one hand, extending the operation window of the DW pinned rotation regime leading to higher DW rotation frequencies and,on the other, affecting anti-symmetrically the dispersion relation in the two domains, thus modulating the SW emission in the direction of electrons flow and allowing propagation in the opposite direction. In other words, the SW emitter can work as unidirectional or asymmetric bidirectional SW emitter depending on the application of an adequate external field. The dipolar field of the rotating DW is the main cause of periodic non-linear excitation of SWs that propagate at higher harmonics of the DW rotation frequency in the system. The displacement of the DW on one side of the notch enhances the excitation on that side and weakens it on the other, giving rise to the unidirectionality. 


\section{Chapter 5}

\section{Conclusions and Outlook}

This thesis has been devoted to the study of magnetic domain wall dynamics in nanostructures such as thin films and nanowires with strong perpendicular magnetic anisotropy. In particular, we have worked on two aspects: the effect of disorder on field driven domain wall dynamics in thin films and a SW emitter based on the pinned rotation of a domain wall at a constriction along a nanowire. Two theoretical tools have been used for this purpose.

On one hand, the one dimensional model simplified the system under study, allowing us to get an understanding on which are the most relevant forces involved in domain wall motion and how they act. By comparing the predictions of this model with experimental data, we have been able to isolate those aspects of DW dynamics that needed clarification, focusing on the characteristics of a system responsible for deviations from the analytical predictions.

On the other, full micromagnetic simulations granted an accurate quantitative description of the dynamics, unravelling the complex magnetization dynamics of large magnetic systems, where the different energy terms compete over different length scales. The development of a customized micromagnetic code made it possible to include in simulations different features needed for the studies performed: spatially varying material parameters to describe disorder, accurate monitoring of energy dissipation at a local level, spatially varying current density and applied fields. Importantly, parallel programming on GPUs granted fast integration times, opening the possibility of systematic studies of domain wall dynamics in large systems.

Advanced micromagnetic simulations and 1D model analytical description of domain wall dynamics have been used in diverse projects within the WALL network, enhancing the interconnection between experimental observations and the underlying theory behind it.

- In the experimental measurement of the efficiency of pure spin current in depinning a DW pinned at a geometrical constriction in a non-local spin valve geometry, the preliminary understanding of the field-driven depinning of the DW was crucial to understand experimental observations. [A. Pfeiffer, 2017] 
- Multi-turn counter sensors based on DW propagation have been on the market for years, but the number of turns that they can count is limited by their geometrical design [5]. They could achieve a potentially unlimited count of turns with a different geometrical design that would prevent their failure. Extensive micromagnetic simulations have been used to optimize the sensor geometry and guide the realization of a potentially infinite turn counter. [B. Borie, 2017]

- Ion irradiation is a novel and promising technique to achieve local control of material properties [131]. In particular, the degree disorder in the material can be tuned via irradiation, leading to different DW dynamics in the creep, depinning and flow regimes. The accurate reproduction of disorder features in micromagnetic simulations is a very useful tool that gives insight into the effects of ion irradiation on DW dynamics, leading to the understanding of the effects of disorder on DW dynamics and on the exploitation of ion irradiation for future applications. [L. Herrera-Diez, in preparation]

- Conventionally, the depinning field is considered independent on the Gilbert damping since it is assumed to be the field at which the Zeeman energy equals the pinning energy barrier (both damping independent). However, the analysis of the domain wall depinning field as a function of the Gilbert damping in a system with perpendicular magnetic anisotropy, performed via full micromagnetic simulations, shows a dependence on the Gilbert damping. In particular, depinning field strongly decreases for small damping parameters. We explain this dependence with the 1D model and we show that the reduction of the depinning field is related to the finite size of the pinning barriers and to the domain wall internal dynamics, which is enhanced for low damping values. [S. Moretti, 2017]

- Understanding the interaction of adjacent domain walls in presence of DMI is an important step in understanding the interaction of neighbouring magnetic bubble and skyrmions. The field driven annihilation of domain walls in hall-cross geometry is used to experimentally investigate the effect of DMI on stabilizing neighbouring homochiral domain walls and prevent their annihilation. 1D model and micromagnetic simulations are used to understand the competition of DMI and dipolar fields in preventing or favouring DW annihilation. [G. V. Kahrnad, in preparation]

Although the work on these projects is intimately related to the development of this thesis and it served as an inspiration and as a stimulus for the research presented, for the sake of conciseness it is not covered in the thesis. The results of such collaborations are presented at page 117 in the list of scientific papers that have been published or that are in preparation. 


\subsection{Domain wall dynamics in disordered systems}

In chapter 3, the need for an understanding of the experimental work on field driven domain wall propagation in $\mathrm{CoFeB}$ thin films led us to study the effect of disorder in these systems and to go beyond the "extrinsic damping" contribution. This explanation, often found in the literature to globally address all energy dissipation mechanisms, does not give insight into the phenomena that lie behind the discrepancy between the dissipated energy in the real system and the prediciton given by analytical models describing DW dynamics. Our explanation involves the internal dynamics of the domain wall, taking place in the precessional regime. In this regime, domain wall velocity drops because the energy injected in the system is not directly converted in domain wall displacement but is stored as kinetic energy of the precessing spins of the domain wall and slowly dissipated. We showed how disorder triggers different spin dynamics that lead to a release of energy from the domain wall into the domains. This transfer of energy to the domains permits a faster energy dissipation to the lattice, since more spins are led to precession in the system and more dissipation channels are accessed.

This result suggests that the estimation of system's parameters from the fitting of one dimensional model to experimental measurements of domain wall motion need to be treated with caution. In fact, as it was shown in chapter 3, the features introduced by disorder cannot simply be included in the existing one dimensional model parameters.

Via micromagnetic simulations we have been able to isolate the contribution of disorder to domain wall velocity in the flow regime and we have shown how that is independent of intrinsic damping parameter and on the applied field. However, an analytical description of these processes is lacking, and an explicit dependence of the additional dissipated power as a function of the external field is missing.

A further step to be taken in this direction would be, on one hand, to describe in a simplified system how the component of dissipated power generated by disorder is not dependent on Gilbert's damping and at least sub-linear with external field. On the other hand, to test this approach experimentally on micron-wide wires; systems that permit field driven domain wall motion at large fields and still allow internal domain wall dynamics.

The study of disordered systems via micromagnetic simulations faces at least two big challenges: on the one hand, disorder in materials shows over many different length scales, from sub-nanometre imperfections such as atomic vacancies and lattice dislocations to surface and thickness inhomogeneities to granular structure, grain boundaries and external impurities. An accurate description of disorder would require a multi-scale software able to resolve the sub-nanometre features while simulating a large system where domain wall related magnetization dynamics can be observed. On the other hand, a statistically significant study of disordered systems requires several realizations of disorder and the introduction of thermal 
agitation increases the number of realizations required for the study. These two requirements cannot be satisfied simultaneously by nowadays simulation tools and computational power, so that a choice need to be made to balance the accuracy of simulations and the reliability of the obtained results. An important help in defining disorder in simulations comes from the experimental insight on the material structure, through which we can determine what feature of disorder is most likely dominant in the magnetic system.

The choice that has been made in our study, that focused on the flow regime in annealed $\mathrm{CoFeB}$, relied on the granular structure and on the dispersion of anisotropy constant and easy axis. However, space dependent damping, reduced exchange constant at the grain boundaries and modulation of saturation magnetization $M_{s}$ to simulate thickness variations could have added accuracy to the study. In particular, the choice of time integration window did not allowed for an accurate description of the creep and depinning regime of domain wall dynamics in the system, which require extremely long time windows [79].

Experimental measurements on the effects of disorder involve the study of DW dynamics in systems exposed to ion irradiation [52]. The intensity of irradiation is, to certain extent, proportional to the amount of disorder introduced in the system. On the other hand, material parameters such as $M_{s}, K_{u}$, damping and DMI constant can be tuned via ion irradiation, allowing a spatial control of material properties that can find applications in DW based memories and sensors.

\subsection{Domain Wall-based Spin Wave emitter}

The second part of the thesis has been devoted to the proposal and study of a new concept of spin wave emitter. In chapter 4 we have presented this concept and proved its functioning and characteristics via micromagnetic simulations in an ideal and optimized system. The study was composed of two steps: first, the one dimensional model was used to understand the pinned precessional dynamics for a domain wall oscillator and lay down the basic geometrical requirements for the working window of the spin waves emitter; the second step involved the systematic study of the dependence of spin wave emission from the injected current.

The systematic micromagnetic study of this kind of problem requires a great computational effort. The analysis of frequency and wave vector spectra require a high time- and space- resolution to explore the high frequency spin wave emission. On the other hand, the self-oscillatory state of a pinned domain wall stabilizes after tens of nanoseconds, requiring long integration time. CUDA-based parallel computing revealed to be fundamental both in the micromagnetic simulation and in the post-processing step.

From the point of view of applications, our work presents three remarkable results

1. Very low current density of operation of the device 
2. The possibility of modulating the signal by changing the applied voltage and by applying an external field

3. The high frequency and coherence of the emitted signal

From a more theoretical point of view, we have explored how coherent spin wave emission is generated from the domain wall by the competition between the shortrange exchange interaction and the long-range dipolar field generated by the rotating domain wall and how this non-linear and periodic interaction leads to spin wave emission at higher odd harmonics of domain wall rotation.

The choice of a very common material for our study on the domain wall spin waves emitter and the relatively simple device structure call for experimental verification of our predictions. Although $\mathrm{CoFeB}$ with low damping was used, recent studies on ultra-low damping [132] CoFe alloy make a good candidate for such system. On the other hand, the detection of domain wall oscillation via anisotropicmagneto-resistance (AMR) signal can be difficult due to the low signal-noise ratio of such small domain wall rotating in a long wire. Detection techniques for spin waves such as micro Brillouin-Light-Scattering ( $\mu \mathrm{BLS}$ ) and indirect current measurements such as inverse spin-hall effect generated by spin pumping in a heavy metal substrate are viable alternative solutions [90].

An important aspect to be considered is disorder: edge roughness deriving from patterning does not affect spin waves propagation. Intrinsic material defects, on the other hand, can oppose domain wall rotation and limit the working window of the device.

Further exploration of the capabilities of this concept of spin wave emitter involves multiple emitters on the same wire, with the objective of amplifying the emitted signal. This can be achieved either by applying a current through a series of pinned domain walls and looking for the superposition of the emitted signals, or by applying the current antiparallel through two notches, looking for a constructive interference in the central region separating them. 



\section{Appendix A}

\section{Voronoi tessellation code}

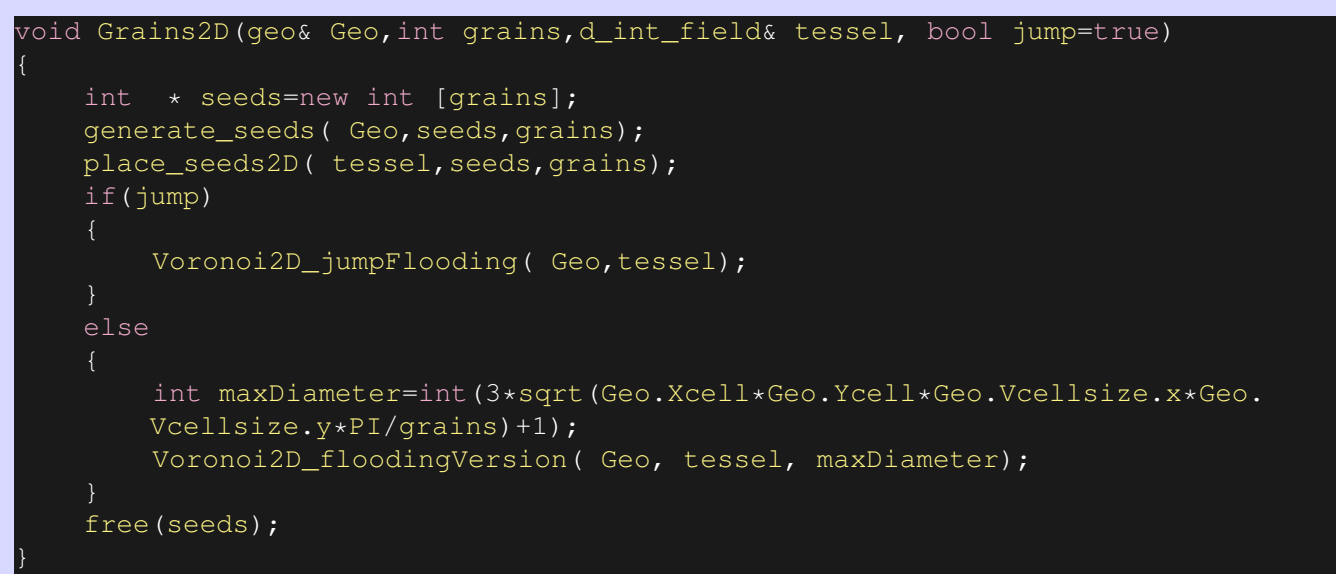

CODE A.1: Function that generates the 2D tessellation of an array of cells.

To generate a Voronoi tessellation of plane, we have to compute the number of grains we want to generate. According to the surface of our sample and the average grain radius $r$ that we desire, the number of grains will be $N_{\text {seeds }}=$ grains $=\frac{S}{\pi r^{2}}$, where $S$ is the surface that we want to tessellate. We generate then $N_{\text {seeds }}$ random numbers between 0 and $N_{\text {cells }}-1$, where $N_{\text {cells }}$ is the total number of mesh points of the discretized sample A.2.

$$
0 \leq s_{i} \leq N_{\text {cells }}-1, \quad i=1, \ldots, N_{\text {seeds }}
$$

We then position the seeds over the sample at the position corresponding to their own value A.3, we do so filling a new array tessel initialized with zeros, and think of the labelling of this array as of the colouring. For now we are colouring with a different color each seed position, leaving blank the rest of the sample.

$$
\text { tessel[seeds[i]]=seeds[i], } i=0, \ldots, N_{\text {seeds }} .
$$

Afterwards, we select the algorithm to use to propagate the colouring throughout the sample, if the choice of the flooding algorithm is made A.5, at every iteration 


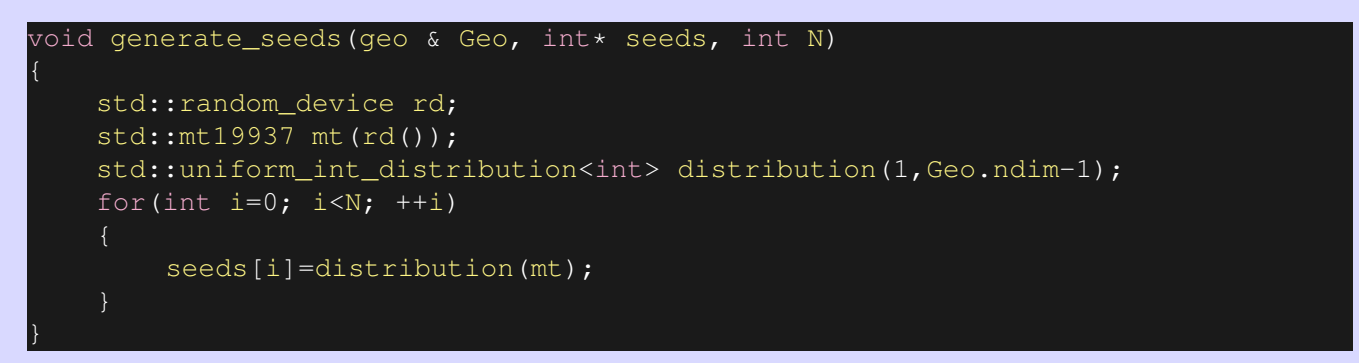

CODE A.2: Generate N random seed positions between cell \#0 and cell \#Geo.ndim-1.

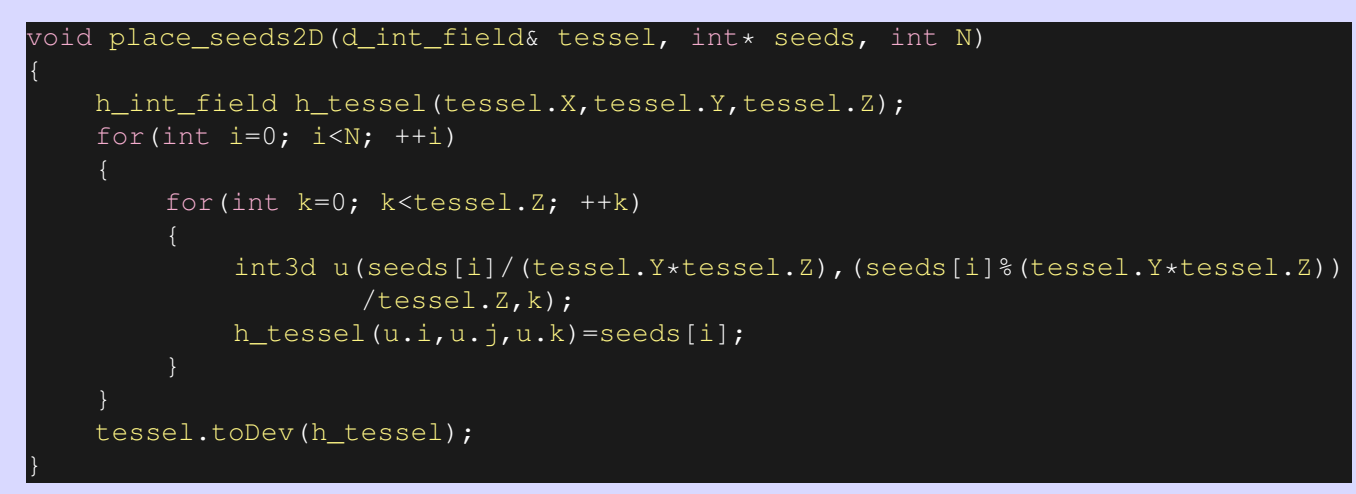

CODE A.3: Place seeds at their mesh grid position in 2D space.

Cells that are seeds are labelled with the number of their position, all other cells are labelled with zero.

the algorithm looks at all cells, if a cell is coloured already, it will look at its neighbours and if a neighbour does not have a color yet, will assign the cell's color to it. If a neighbour cell is coloured, then the colour tells us the position of the seed, the algorithm then measures to which of the two seeds the cell in question is closer, using the function A.4, and updates its color accordingly. The jump-flooding algorithm A.6 works similarly, except that, once a coloured cell is found, instead of looking at its adjacent cells, the algorithm jumps at a distance xJump or y Jump and there repeats the color comparison procedure. This distance is large at the beginning at is reduced by half at every iteration.

In the presented code, the tessellation is performed in the two planar dimensions, a similar code that expands the colouring also along the $z$-direction can be used to generate 3D grains in bulk samples. 


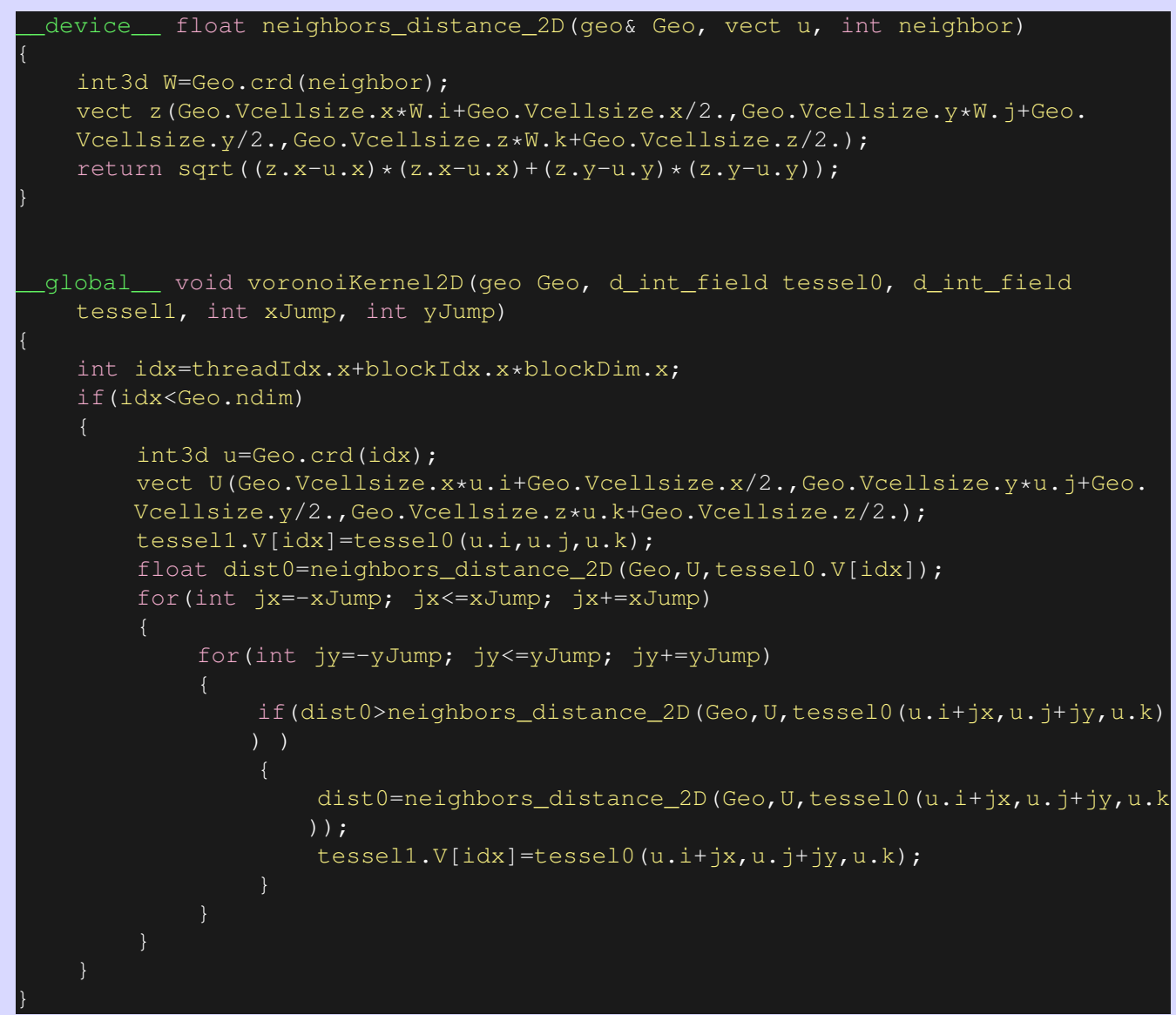

CODE A.4: Kernel function that checks the four cells at distance $\pm \mathrm{xJump}, \pm \mathrm{y}$ Jump from each target cell. The target cell is re-labelled with the label of the closest seed between the four labels checked.

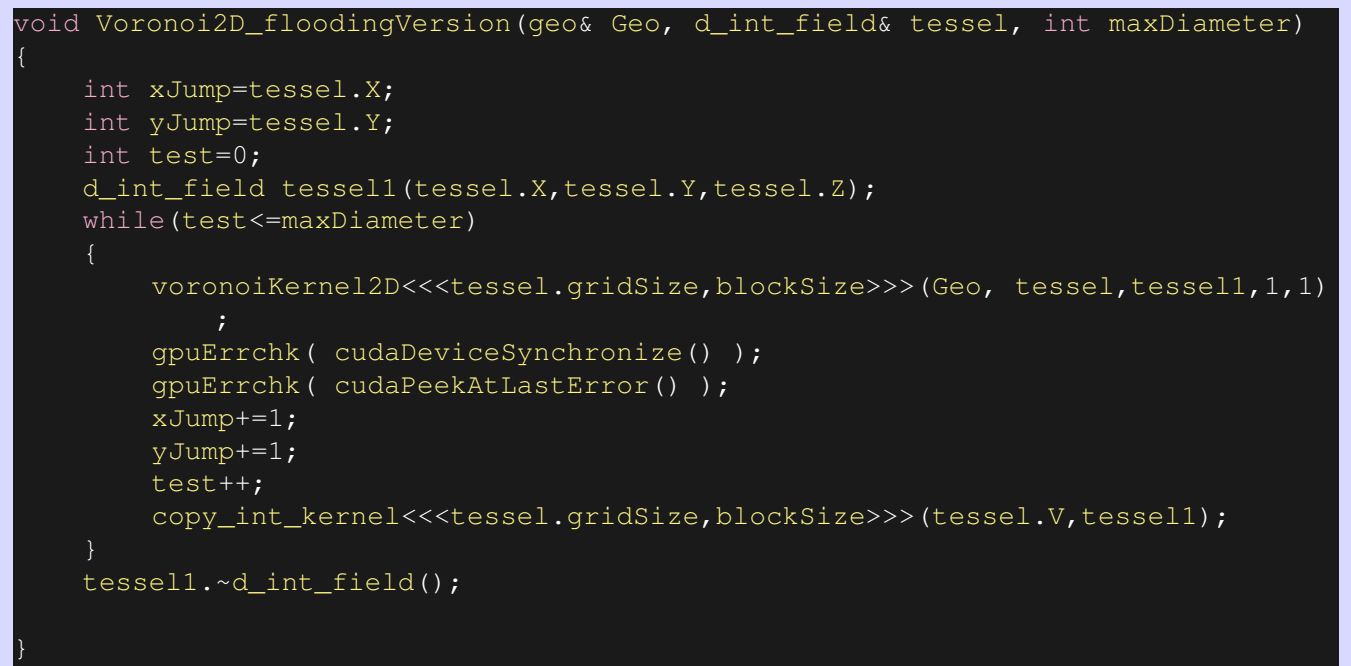

CODE A.5: Flooding algorithm. Each cell accesses the four neighboring ones. 


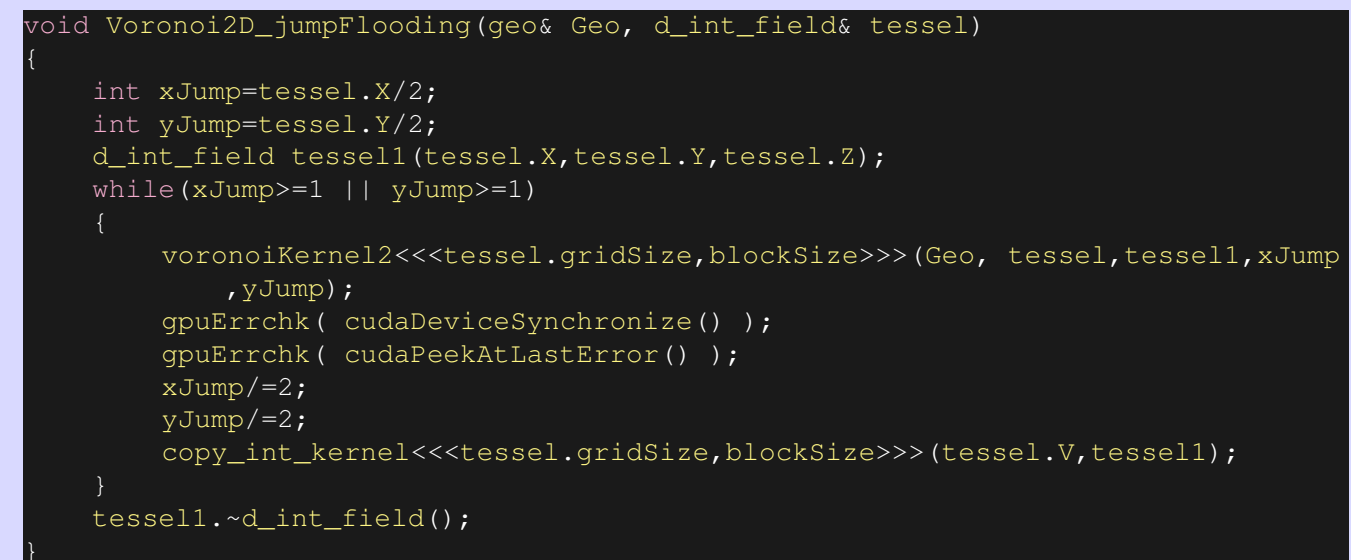

CODE A.6: Jump-flooding algorithm. Each cell is compared with four cells distant from it half of the total size, then one quarter and so on. 


\section{List of publications}

- M. Voto, L. Lopez-Diaz, and L. Torres, "Effects of grain size and disorder on domain wall propagation in CoFeB thin films," J. Phys. D. Appl. Phys., vol. 49, no. 18, p. 185001, May 2016.

- M. Voto, L. Lopez-Diaz, L. Torres, and S. Moretti, "Disorder-induced domain wall velocity shift at high fields in perpendicularly magnetized thin films," Phys. Rev. B, vol. 94, no. 17, p. 174438, Nov. 2016.

- M. Voto, L. Lopez-Diaz, and E. Martinez, "Pinned domain wall oscillator as a tuneable direct current spin wave emitter," Sci. Rep., vol. 7, no. 1, p. 13559, Oct. 2017.

- A. Pfeiffer, R. M. Reeve, M. Voto, W. Savero-Torres, N. Richter, L. Vila, J.-P. Attane, L. Lopez-Diaz, and M. Kläui, "Geometrical control of pure spin current induced domain wall depinning," J. Phys. Condens. Matter, vol. 29, no. 8, p. 85802, Mar. 2017.

- S. Moretti, M. Voto, and E. Martinez, "Dynamical depinning of chiral domain walls," Phys. Rev. B, vol. 96, no. 5, p. 54433, Aug. 2017.

- B. Borie, M. Voto, L. Lopez-Diaz, H. Grimm, M. Diegel, M. Kläui, and R. Mattheis, "Reliable Propagation of Magnetic Domain Walls in Cross Structures for Advanced Multiturn Sensors," Phys. Rev. Appl., vol. 8, no. 4, p. 44004, Oct. 2017.

- L. Herrera Diez, G. Durin, V. Jeudy, Y. Liu, B. Sarma, M. Voto, G. Agnus, D. Bouville, L. Vila, J. Langer, B. Ocker, L. Lopez-Diaz, and D. Ravelosona, “Creep motion of magnetic domain walls in $\mathrm{CoFeB} / \mathrm{MgO}$ microstructures," In preparation.

- L. Herrera Diez, M. Voto, M. Belmeguenai, A. Casiraghi, G. Durin, V. Jeudy, V. Sluka, A. D. Kent, T. Devolder, A. Lamperti, R. Mantovan, G. Agnus, J. Langer, B. Ocker, Lopez- Dias and D. Ravelosona, "Magnetic domain wall motion beyond creep: defects and interface engineering," In preparation.

- Gurucharan V. Karnad, Eduardo Martinez, M. Voto, Tomek Schulz, and Mathias Kläui, "Study of energetics of $360^{\circ}$ domain walls through annihilation," In preparation. 



\section{Bibliography}

[1] S. Blundell and D. Thouless. Magnetism in Condensed Matter. Vol. 71. 1. Oxford University press, 2003, pp. 94-95. DOI: $10.1119 / 1.1522704$ (cit. on pp. 1, $5-7,11,12)$.

[2] W. Brown. Micromagnetics. Interscience, 1963 (cit. on pp. 1, 11, 35, 40).

[3] D. Awschalom. Handbook of Spintronics. Ed. by Y. Xu, D. D. Awschalom, and J. Nitta. Dordrecht: Springer Netherlands, 2016. DOI: 10 . 1007 / $978-94-$ 007-6892-5 (cit. on p. 1).

[4] J. Akerman. "APPLIED PHYSICS: Toward a Universal Memory". Science 308.5721 (2005), pp. 508-510. DOI: $10.1126 /$ science. 1110549 (cit. on p. 2).

[5] R. Weiss, R. Mattheis, and G. Reiss. "Advanced giant magnetoresistance technology for measurement applications". Measurement Science and Technology 24 (2013), p. 082001. DOI: 10 .1088/0957-0233/24/8/082001 (cit. on pp. 2, $47,108)$.

[6] S. Parkin and S.-H. Yang. "Memory on the racetrack". Nature Nanotechnology 10.3 (2015), pp. 195-198. DOI: 10.1038 /nnano. 2015.41 (cit. on p. 2).

[7] R. L. Stamps et al. "The 2014 Magnetism Roadmap". Journal of Physics D: Applied Physics 47 (2014), p. 333001. DOI: 10 . 1088 / 0022 - 3727 / 47 / 33 / 333001 . arXiv: 1410.6404 (cit. on pp. 2,77).

[8] D. A. Allwood et al. "Magnetic domain-wall logic." Science 309.2005 (2005), pp. 1688-1692. DOI: $10.1126 /$ science. 1108813 (cit. on pp. 2, 47).

[9] C. Burrowes et al. "Low depinning fields in Ta-CoFeB-MgO ultrathin films with perpendicular magnetic anisotropy". Applied Physics Letters 103.18 (2013), p. 182401. DOI: $10.1063 / 1.4826439$ (cit. on pp. 2, 47-49, 51, 52, 54, 56, 59, $65)$.

[10] A. Hrabec et al. "Measuring and tailoring the Dzyaloshinskii-Moriya interaction in perpendicularly magnetized thin films". Physical Review B 90.2 (July 2014), p. 020402. DOI: 10.1103 /PhysRevB. 90.020402 (cit. on p. 2).

[11] S. Lemerle et al. "Domain Wall Creep in an Ising Ultrathin Magnetic Film". Physical Review Letters 80.4 (1998), pp. 849-852. DOI: 10 . 1103 / PhysRevLett . 80.849 (cit. on pp. $2,48,51,56$ ). 
[12] V. Jeudy et al. "Universal Pinning Energy Barrier for Driven Domain Walls in Thin Ferromagnetic Films". Physical Review Letters 117.5 (2016), pp. 1-5. DOI: 10.1103 / PhysRevLett . 117 .057201. arXiv: arXiv: 1603.01674v1 (cit. on pp. 2, 50, 51).

[13] B. Hillebrands and K. Ounadjela, eds. Spin Dynamics in Confined Magnetic Structures I. Vol. 83. Topics in Applied Physics. Berlin, Heidelberg: Springer Berlin Heidelberg, 2002. DOI: 10 . 1007/3-540-40907-6 (cit. on pp. 2, 5, $47,64)$.

[14] L Lopez-Diaz et al. "Micromagnetic simulations using Graphics Processing Units". Journal of Physics D: Applied Physics 45.32 (2012), p. 323001. DOI: 10 . $1088 / 0022-3727 / 45 / 32 / 323001$ (cit. on pp. 2,31,36, 44).

[15] D. Stancil and A. Prabhakar. Spin Waves. Boston, MA: Springer US, 2009, p. 364. DOI: $10.1007 / 978-0-387-77865-5$ (cit. on pp. 5,77 ).

[16] G. Bertotti. Hysteresis in Magnetism: For Physicists, Materials Scientists, and Engineers. Academic Press, 1998, p. 558 (cit. on pp. 5, 9).

[17] A. Hubert and R. Schäfer. 1.1 What are Magnetic Domains? Springer Verlag, 2009, p. 441. DOI: 10 .1007/978-3-540-85054-0 (cit. on pp. 5, 22, 31).

[18] G. Albuquerque. "Precession de l'aimantation en geometrie confinee: aspects physiques et numeriques". 2002 (cit. on p. 11).

[19] A. Aharoni and A. Arrott. Introduction to the Theory of Ferromagnetism. Vol. 50. 9. 1997, pp. 66-68. DOI: $10.1063 / 1.881916$ (cit. on pp. 11, 19, 31).

[20] K. Nakamura et al. "Role of an interfacial FeO layer in the electric-fielddriven switching of magnetocrystalline anisotropy at the Fe/MgO interface". Physical Review B - Condensed Matter and Materials Physics 81.22 (2010), pp. 14. DOI: 10.1103 /PhysRevB. 81.220409 (cit. on p. 13).

[21] S. Ikeda et al. "A perpendicular-anisotropy CoFeB-MgO magnetic tunnel junction". Nature Materials 9.9 (2010), pp. 721-724. DOI: 10 . 1038 / nmat2804 (cit. on pp. 13, 47).

[22] A. Thiaville et al. "Dynamics of Dzyaloshinskii domain walls in ultrathin magnetic films". Europhysics Letters 100.5 (2012), p. 57002. DOI: 10 . 1209 / 0295-5075/100/57002 (cit. on p. 15).

[23] S. Rohart and a. Thiaville. "Skyrmion confinement in ultrathin film nanostructures in the presence of Dzyaloshinskii-Moriya interaction". Physical Review B - Condensed Matter and Materials Physics 88.18 (2013), pp. 1-8. DOI: 10.1103/PhysRevB. 88.184422 . arXiv: 1310.0666 (cit. on p. 16).

[24] B. Hillebrands and A. Thiaville, eds. Spin Dynamics in Confined Magnetic Structures III. Vol. 101. Topics in Applied Physics. Springer Berlin Heidelberg, 2006. DOI: $10.1007 /$ b12462 (cit. on p. 28). 
[25] W. Press et al. Numerical Recipes: The Art of Scientific Computing. Vol. 29. 4. 1987, p. 501. DOI: $10.2307 / 1269484$. arXiv: $1011.1669 v 3$ (cit. on pp. 31, 35).

[26] J. E. Miltat and M. J. Donahue. "Numerical Micromagnetics: Finite Difference Methods". Handbook of Magnetism and Advanced Magnetic Materials. Chichester, UK: John Wiley \& Sons, Ltd, 2007. DOI: 10 . 1002 / 9780470022184 . hmm202 (cit. on p. 31).

[27] M. J. Donahue and D. G. Porter. OOMMF User's Guide, Version 1.0. 1999. DOI: 10. 4231 /D3XS5JJ23 (cit. on pp. 31, 39, 41).

[28] A. Vansteenkiste et al. "The design and verification of MuMax3". AIP Advances 4.10 (2014), pp. 0-22. DOI: $10.1063 / 1.4899186$. arXiv: 1406.7635 (cit. on pp. 31, 39).

[29] GPMagnet, http://www.goparallel.net (cit. on p. 31).

[30] C. Johnson. Numerical solutions of partial differential equations by the finite element method. 1987. DOI: 10 .1016/S0167-5648(08)70020-0 (cit. on p. 31).

[31] Magpar, http://www.magpar.net/ (cit. on p. 31).

[32] Nmag, URL: http://nmag.soton.ac.uk/nmag/(cit. on p. 31).

[33] A. J. Newell, W. Williams, and D. J. Dunlop. "A generalization of the demagnetizing tensor for nonuniform magnetization". Journal of Geophysical Research 98.B6 (1993), p. 9551. DOI: 10 . 1029/93 JB0 0694 (cit. on p. 35).

[34] W. F. Brown. "Thermal fluctuations of a single-domain particle". Physical Review 130.5 (1963), pp. 1677-1686. DOI: 10.1103 / PhysRev . 130 . 1677. arXiv: arXiv:1011.1669v3 (cit. on p.36).

[35] E. Martínez. "On the interpretations of Langevin stochastic equation in different coordinate systems". Physica B: Condensed Matter 343.1-4 (2004), pp. 252256. DOI: $10.1016 / j$.physb. 2003.08 .103 (cit. on p. 36).

[36] R. L. Burden and J. D. Faires. Numerical Analysis. 2011, p. 888. DOI: $10.1017 /$ CB09781107415324.004. arXiv: arXiv:1011.1669v3 (cit. on p. 39).

[37] J. Dormand and P. Prince. "A family of embedded Runge-Kutta formulae". Journal of Computational and Applied Mathematics 6.1 (1980), pp. 19-26. DOI: 10.1016/0771-050X (80) 90013-3 (cit. on p. 39).

[38] J. R. Dormand and P. J. Prince. "A reconsideration of some embedded formulae". Journal of Computational and Applied Mathematics 15 (1986), pp. 203-211. DOI: $10.1016 / 0377-0427$ (86) 90027-0 (cit. on pp. 39, 93).

[39] J. C. Butcher. Numerical methods for ordinary differential equations. John Wiley \& Sons, 2008 (cit. on p. 39).

[40] CUDA C porgramming guide (cit. on p. 42). 
[41] H. Sutter. "The Free lLunch Is Over". C/C++ Users Journal (2005) (cit. on p. 43).

[42] K. Wong and H. A. Müller. An Efficient Implementation of Fortune's Plane-Sweep Algorithm for Voronoi Diagrams (cit. on p. 44).

[43] G. Rong and T.-s. Tan. "Jump flooding in GPU with applications to Voronoi diagram and distance transform". in Studies in Logical Theory, American Philosophical Quarterly Monograph 2 (2006), pp. 109-116. DOI: 10 . 1145/1111411. 1111431 (cit. on p. 45).

[44] G. Rong et al. "GPU-assisted computation of centroidal voronoi tessellation". IEEE Transactions on Visualization and Computer Graphics 17.3 (2011), pp. 345356. DOI: $10.1109 /$ TVCG. 2010.53 (cit. on p. 45).

[45] S. Parkin, M. Hayashi, and L. Thomas. "Magnetic Domain-Wall Racetrack Memory". Science 320.5873 (2008), pp. 190-194. DOI: 10 . 1126 / science . 1145799 (cit. on p. 47).

[46] M. Hayashi et al. “Current-Controlled Magnetic Domain-Wall Nanowire Shift Register". Science 320.5873 (2008), pp. 209-211. DOI: 10.1126 / science. 1154587 (cit. on p. 47).

[47] A. V. Khvalkovskiy et al. "Basic principles of STT-MRAM cell operation in memory arrays". Journal of Physics D: Applied Physics 46.7 (2013), p. 074001. DOI: $10.1088 / 0022-3727 / 46 / 7 / 074001$ (cit. on p. 47).

[48] C. Bilzer et al. "Study of the dynamic magnetic properties of soft CoFeB films". Journal of Applied Physics 100.5 (2006), p. 053903. DOI: 10 . 1063 / 1. 2337165 (cit. on p. 47).

[49] N. L. Schryer and L. R. Walker. "The motion of $180^{\circ}$ domain walls in uniform dc magnetic fields". Journal of Applied Physics 45.12 (1974), pp. 5406-5421. DOI: $10.1063 / 1.1663252$ (cit. on p. 47).

[50] A. Malozemoff and J. Slonczewski. Magnetic domain walls in bubble materials. 1979. DOI: $10.1016 /$ B978-0-12-002951-8.50014-5 (cit. on pp. 47, 48, $65,66,68,69)$.

[51] P. J. Metaxas et al. "Creep and Flow Regimes of Magnetic Domain-Wall Motion in Ultrathin $\mathrm{Pt} / \mathrm{Co} / \mathrm{Pt}$ Films with Perpendicular Anisotropy". Physical Review Letters 99.21 (2007), p. 217208. DOI: 10 . 1103 / PhysRevLett . 99 . 217208 (cit. on pp. 47, 48, 56, 59).

[52] L. Herrera Diez et al. "Controlling magnetic domain wall motion in the creep regime in $\mathrm{He}+$-irradiated $\mathrm{CoFeB} / \mathrm{MgO}$ films with perpendicular anisotropy". Applied Physics Letters 107.3 (2015), p. 032401. DOI: 10 . 1063 / 1 . 4927204 (cit. on pp. 48, 49, 54-56, 93, 110).

[53] P. Chauve, T. Giamarchi, and P. Le Doussal. "Creep and depinning in disordered media". Physical Review B 62.10 (2000), pp. 6241-6267. DOI: 10.1103 / PhysRevB. 62.6241 (cit. on pp. 48, 56). 
[54] K.-J. Kim et al. "Interdimensional universality of dynamic interfaces." Nature 458.7239 (2009), pp. 740-2. DOI: 10.1038 / nature 07874 (cit. on p. 48).

[55] A. Larkin and A. Varlamov. "Theory of Fluctuations in Superconductors." Journal of Statistical Physics 123.2 (2006), pp. 497-498. DOI: 10 . 1007 / s 10955 005-9007-5 (cit. on pp. 48, 51).

[56] K. Yamada et al. "Influence of Instabilities on High-Field Magnetic Domain Wall Velocity in (Co/Ni) Nanostrips". Applied Physics Express 4.11 (2011), p. 113001. DOI: 10.1143/APEX. 4.113001 (cit. on pp. 48, 49, 56, 59).

[57] T. Weindler et al. "Magnetic Damping: Domain Wall Dynamics versus Local Ferromagnetic Resonance". Physical Review Letters 113.23 (2014), p. 237204. DOI: 10.1103 /PhysRevLett.113.237204 (cit. on pp. 49,65).

[58] K. Yamada and Y. Nakatani. "Excitation of magnetic domain wall velocity in (Co/Ni) nanowires induced by blocking the motion of vertical Bloch lines". Applied Physics Express 8.9 (2015), p. 093004. DOI: 10.7567 /APEX. 8.093004 (cit. on pp. 49, 68, 69, 71).

[59] Y. Yoshimura et al. "Soliton-like magnetic domain wall motion induced by the interfacial Dzyaloshinskii-Moriya interaction". Nature Physics 12.2 (2015), pp. 157-161. DOI: $10.1038 /$ nphys 3535. arXiv: 1508.00197 (cit. on pp. 49, $68,71)$.

[60] H. Min et al. "Effects of Disorder and Internal Dynamics on Vortex Wall Propagation". Physical Review Letters 104.21 (2010), p. 217201. DOI: 10.1103 / PhysRevLett. 104.217201 (cit. on pp. 49, 52, 59, 60, 65, 73).

[61] J. Leliaert et al. “Current-driven domain wall mobility in polycrystalline Permalloy nanowires: A numerical study". Journal of Applied Physics 115.23 (2014), p. 233903. DOI: $10.1063 / 1$. 4883297 (cit. on pp. 49, 52, 59, 60, 62, 65).

[62] G. Blatter et al. "Vortices in high-temperature superconductors". Reviews of Modern Physics 66.4 (1994), pp. 1125-1388. DOI: 10 . 1103 / RevModPhys . 66.1125 (cit. on p. 51).

[63] P. J. Metaxas. "Creep and Flow Dynamics of Magnetic Domain Walls". Solid State Physics - Advances in Research and Applications. Ed. by C. \&. Stamps. Vol. 62. 2010, pp. 75-162. DOI: 10.1016/B978-0-12-374293-3.00002-X (cit. on pp. 51, 56).

[64] J. Gorchon et al. "Pinning-dependent field-driven domain wall dynamics and thermal scaling in an ultrathin $\mathrm{Pt} / \mathrm{Co} / \mathrm{Pt}$ magnetic film". Physical Review Letters 113.2 (2014), pp. 1-5. DOI: 10.1103 / PhysRevLett. 113.027205. arXiv: 1407.7781 (cit. on p. 51).

[65] B. Van De Wiele, L. Laurson, and G. Durin. "Effect of disorder on transverse domain wall dynamics in magnetic nanostrips". Physical Review B - Condensed Matter and Materials Physics 86.14 (2012), pp. 1-5. DOI: 10.1103 / PhysRevB . 86.144415. arXiv: 1209.5274 (cit. on p. 52). 
[66] B. Van De Wiele, L. Laurson, and G. Durin. "The role of disorder in the domain wall dynamics of magnetic nanostrips". European Physical Journal B 86.3 (2013). DOI: $10.1140 /$ epjb/e2012-30674-0 (cit. on p. 52).

[67] J. Leliaert et al. "A numerical approach to incorporate intrinsic material defects in micromagnetic simulations". Journal of Applied Physics 115.17 (2014), p. 17D102. DOI: $10.1063 / 1.4854956$ (cit. on p. 52).

[68] J. Leliaert et al. "Influence of material defects on current-driven vortex domain wall mobility". Physical Review B 89.6 (2014), p. 064419. DOI: 10.1103 / PhysRevB. 89.064419 (cit. on p. 52).

[69] R. A. Khan et al. "Effect of annealing on the interfacial Dzyaloshinskii-Moriya interaction in Ta/CoFeB/MgO trilayers". Applied Physics Letters 109.13 (2016). DOI: $10.1063 / 1.4963731$. arXiv: 1607.06405 (cit. on p. 52).

[70] T. Takeuchi et al. "Crystallization of amorphous CoFeB ferromagnetic layers in $\mathrm{CoFeB} / \mathrm{MgO} / \mathrm{CoFeB}$ magnetic tunnel junctions". Japanese Journal of Applied Physics, Part 2: Letters 46.25-28 (2007). DOI: 10 . 1143 / JJAP . 46 . L623 (cit. on p. 52).

[71] Y. T. Chen and C. C. Chang. "Effect of grain size on magnetic and nanomechanical properties of Co60Fe20B20 thin films". Journal of Alloys and Compounds 498.2 (2010), pp. 113-117. DOI: $10.1016 /$ j. jallcom. 2010.03 . 141 (cit. on p. 52).

[72] H. Meng et al. "Annealing effects on CoFeB-MgO magnetic tunnel junctions with perpendicular anisotropy". Journal of Applied Physics 110.3 (2011), p. 033904. DOI: $10.1063 / 1.3611426$ (cit. on p. 52).

[73] J. W. S. Rayleigh. "The Problem of the Random Walk". Nature 72 (1905), p. 318. DOI: $10.1038 / 072318 \mathrm{a} 0$ (cit. on p. 54).

[74] R. Lo Conte et al. "Role of B diffusion in the interfacial Dzyaloshinskii-Moriya interaction in $\mathrm{Ta} / \mathrm{Co}_{20} \mathrm{Fe}_{60} \mathrm{~B}_{20} / \mathrm{MgO}$ nanowires". Physical Review B 91.1 (Jan. 2015), p. 014433. DOI: 10.1103 / PhysRevB. 91.014433 (cit. on p. 54).

[75] J.-P. Tetienne et al. "The nature of domain walls in ultrathin ferromagnets revealed by scanning nanomagnetometry". Nature Communications 6 (2015), p. 6733. DOI: 10.1038 /ncomms 7733. arXiv: 1401.6722 (cit. on p. 54).

[76] A. Mougin et al. "Domain wall mobility, stability and Walker breakdown in magnetic nanowires". Europhysics Letters (EPL) 78.5 (2007), p. 57007. DOI: 10 . 1209/0295-5075/78/57007 (cit. on p. 56).

[77] T. Herranen and L. Laurson. "Domain walls within domain walls in wide ferromagnetic strips". Physical Review B 92.10 (2015), p. 100405. DOI: 10.1103 / PhysRevB. 92.100405 (cit. on pp. 56, 57, 60, 65, 68, 71).

[78] J. Żebrowski and A. Sukiennicki. J. Appl. Phys. 52.6 (1981), pp. 4176-4180. DOI: http://dx.doi.org/10.1063/1.329230 (cit. on p.56). 
[79] J. Leliaert et al. "Creep turns linear in narrow ferromagnetic nanostrips." Scientific reports 6 (2016), p. 20472. DOI: 10 . 1038 / srep20 472 (cit. on pp. 56, 110).

[80] D.-T. Ngo et al. "Perpendicular magnetic anisotropy and the magnetization process in $\mathrm{CoFeB} / \mathrm{Pd}$ multilayer films". Journal of Physics D: Applied Physics 47.44 (2014), p. 445001. DOI: $10.1088 / 0022-3727 / 47 / 44 / 445001$ (cit. on pp. 59, 62).

[81] C. Park et al. "Influence of Boron Diffusion on Transport and Magnetic Properties in $\mathrm{CoFeB} / \mathrm{MgO} / \mathrm{CoFeB}$ Magnetic Tunnel Junction". IEEE Transactions on Magnetics 45.10 (2009), pp. 3457-3459. DOI: 10 . 1109 / TMAG . 2009 . 2022495 (cit. on pp. 59, 62).

[82] M. Voto, L. Lopez-Diaz, and L. Torres. "Effects of grain size and disorder on domain wall propagation in CoFeB thin films". Journal of Physics D: Applied Physics 49.18 (2016), p. 185001. DOI: $10.1088 / 0022-3727 / 49 / 18 / 185001$ (cit. on p. 65).

[83] H.-B. Braun. "Topological effects in nanomagnetism: from superparamagnetism to chiral quantum solitons". Advances in Physics 61.1 (2012), pp. 1116. DOI: $10.1080 / 00018732.2012 .663070$ (cit. on p. 69).

[84] R. Wieser, E. Y. Vedmedenko, and R. Wiesendanger. "Domain wall motion damped by the emission of spin waves". Physical Review B 81.2 (2010), p. 024405. DOI: 10.1103 /PhysRevB. 81.024405 (cit. on pp. 73, 78).

[85] X. S. Wang et al. "Domain wall propagation through spin wave emission". Physical Review Letters 109.16 (2012), pp. 1-4. DOI: 10.1103 / Phys RevLett . 109.167209. arXiv: arXiv:1206.6244v1 (cit. on pp. 73, 78).

[86] X. S. Wang and X. R. Wang. "Spin wave emission in field-driven domain wall motion". Physical Review B - Condensed Matter and Materials Physics 90.18 (2014), pp. 1-6. DOI: 10 . 1103 / PhysRevB . 90 . 184415 . arXiv: arXiv : $1409.3286 \mathrm{v} 2$ (cit. on p. 73).

[87] Y. Nakatani, A. Thiaville, and J. Miltat. "Faster magnetic walls in rough wires." Nature materials 2.8 (2003), pp. 521-523. DOI: 10 . 1038 / nmat 931 (cit. on p. 73).

[88] X. R. Wang, P. Yan, and J. Lu. "Field-induced domain wall propagation velocity in magnetic nanowires". Europhysics Letters (EPL) 86.67001 (2009), pp. 1-5. DOI: $10.1209 / 0295-5075 / 86 / 67001$. arXiv: 0903.0431 (cit. on p. 74).

[89] V. V. Kruglyak, S. O. Demokritov, and D Grundler. "Magnonics". Journal of Physics D: Applied Physics 43 (2010), p. 264001. DOI: 10.1088 / $0022-3727$ / 43/26/264001. arXiv: /arxiv.org/abs/1101.0479 [http:] (cit. on p. 77).

[90] A. V. Chumak et al. “Magnon spintronics”. Nature Physics 11.6 (2015), pp. 453461. DOI: $10.1038 /$ nphys 3347 (cit. on pp. 77,111 ). 
[91] A. V. Chumak, A. a. Serga, and B. Hillebrands. "Magnon transistor for allmagnon data processing". Nature Communications 5.May 2013 (2014), p. 4700. DOI: $10.1038 /$ ncomms 5700 (cit. on p. 77).

[92] K. Vogt et al. "Realization of a spin-wave multiplexer". Nature Communications 5 (2014), pp. 1-5. DOI: $10.1038 /$ ncomms 4727 (cit. on p. 77).

[93] A. Khitun, M. Bao, and K. L. Wang. "Magnonic logic circuits". Journal of Physics D: Applied Physics 43.26 (2010), p. 264005 (cit. on p. 77).

[94] T. Schneider et al. "Realization of spin-wave logic gates". Applied Physics Letters 92.2 (2008). DOI: $10.1063 / 1.2834714$. arXiv: 0711.4720 (cit. on p. 77).

[95] S. Klingler et al. "Spin-wave logic devices based on isotropic forward volume magnetostatic waves". Applied Physics Letters 106.21 (2015). DOI: 10 . 1063 / 1.4921850. arXiv: arXiv:1503.04101v1 (cit. on p. 77).

[96] A. V. Chumak et al. "Direct detection of magnon spin transport by the inverse spin Hall effect". Applied Physics Letters 100.8 (2012), pp. 1-4. DOI: 10.1063 / 1.3689787. arXiv: 1112.4969 (cit. on p. 77).

[97] F. B. Mushenok et al. "Broadband conversion of microwaves into propagating spin waves in patterned magnetic structures". Applied Physics Letters 111.4 (2017), p. 042404. DOI: $10.1063 / 1.4995991$ (cit. on p. 77).

[98] N. J. Whitehead et al. "Theory of linear spin wave emission from a Bloch domain wall". Physical Review B 96.6 (2017), p. 064415. DOI: 10 . 1103 / Phy sRevB . 96.064415. arXiv: 1705.01852 (cit. on pp. 77, 78).

[99] V. E. Demidov, S. Urazhdin, and S. O. Demokritov. "Direct observation and mapping of spin waves emitted by spin-torque nano-oscillators." Nature materials 9.12 (2010), pp. 984-988. DOI: 10.1038 /nmat 2882 (cit. on p. 77).

[100] M Madami et al. "Direct observation of a propagating spin wave induced by spin-transfer torque". Nat Nanotechnol 6.10 (2011), pp. 635-638. DOI: 10 . 1038/nnano.2011.140 (cit. on p. 77).

[101] V. E. Demidov et al. "Magnetic nano-oscillator driven by pure spin current". Nature Materials 11.12 (2012), pp. 1028-1031. DOI: 10 . 1038 / nmat 3459 (cit. on p. 77).

[102] Y. Kajiwara et al. "Transmission of electrical signals by spin-wave interconversion in a magnetic insulator". Nature 464.7286 (2010), pp. 262-266. DOI: $10.1038 /$ nature08876. arXiv: 1302.1352 (cit. on p. 77).

[103] P. Rovillain et al. "Electric-field control of spin waves at room temperature in multiferroic BiFeO3". Nature Materials 9.12 (2010), pp. 975-979. DOI: 10 . 1038 /nmat2899. arXiv: 1010.2678 (cit. on p. 77). 
[104] M. Weiler et al. "Elastically driven ferromagnetic resonance in nickel thin films". Physical Review Letters 106.11 (2011), pp. 1-4. DOI: 10 . 1103/Phy sRevLett. 106.117601. arXiv: arXiv:1009.5798v2 (cit. on p. 77).

[105] S. Cherepov et al. "Electric-field-induced spin wave generation using multiferroic magnetoelectric cells". Applied Physics Letters 104.8 (2014). DOI: 10 . $1063 / 1.4865916$ (cit. on p. 77).

[106] L. Berger. "Possible existence of a Josephson effect in ferromagnets". Physical Review B 33.3 (1986), pp. 1572-1578. DOI: 10 . 1103 / PhysRevB . 33.1572 (cit. on pp. 77, 79).

[107] J. Slonczewski. "Current-driven excitation of magnetic multilayers". Journal of Magnetism and Magnetic Materials 159.1-2 (1996), pp. L1-L7. DOI: 10.1016 / $0304-8853$ (96) $00062-5$ (cit. on p. 77).

[108] S. Zhang and Z. Li. "Roles of nonequilibrium conduction electrons on the magnetization dynamics of ferromagnets". Physical Review Letters 93.12 (2004), pp.1-4. DOI: 10.1103/PhysRevLett.93.127204.arXiv: 0407174 [cond-mat] (cit. on pp. 77, 79).

[109] R. H. Liu, W. L. Lim, and S. Urazhdin. "Spectral characteristics of the microwave emission by the spin hall nano-oscillator". Physical Review Letters 110.14 (2013), pp. 1-5. DOI: 10.1103/PhysRevLett.110.147601. arXiv: 1210.2758 (cit. on p. 77).

[110] A. Hamadeh et al. "Full control of the spin-wave damping in a magnetic insulator using spin-orbit torque". Physical Review Letters 113.19 (2014), pp. 15. DOI: 10.1103 / PhysRevLett.113.197203. arXiv: 1405.7415 (cit. on p. 77).

[111] A. A. Awad et al. "Long-range mutual synchronization of spin Hall nanooscillators". Nature Physics 1.November (2016), pp. 1-4. DOI: 10 . 1038 / nphys 3927 (cit. on p. 77).

[112] D. Bouzidi and H. Suhl. "Motion of a Bloch domain wall". Physical Review Letters 65.20 (1990), pp. 2587-2590. DOI: 10.1103 / Phys RevLett . 65.2587 (cit. on pp. 78, 103).

[113] H. Xia et al. "Doppler effect in a solid medium: Spin wave emission by a precessing domain wall drifting in spin current". Physical Review B - Condensed Matter and Materials Physics 93.14 (2016), pp. 1-5. DOI: 10 . 1103 /PhysRevB . 93.140410 (cit. on pp. 78, 101).

[114] Y. Le Maho, J. V. Kim, and G. Tatara. "Spin-wave contributions to currentinduced domain wall dynamics". Physical Review B - Condensed Matter and Materials Physics 79.17 (2009). DOI: 10.1103/PhysRevB. 79.174404 . arXiv: 0812.4084 (cit. on pp. 78, 103). 
[115] B. Van de Wiele et al. "Tunable short-wavelength spin wave excitation from pinned magnetic domain walls". Scientific Reports 6.August 2015 (2016), p. 21330. DOI: $10.1038 /$ srep21330 (cit. on p. 78).

[116] T. Ono and Y. Nakatani. "Magnetic domain wall oscillator". Applied Physics Express 1.6 (2008), pp. 0613011-0613013. DOI: 10 . 1143 / APEX . 1.061301 (cit. on pp. 78,97$)$.

[117] A. Bisig et al. "Tunable steady-state domain wall oscillator with perpendicular magnetic anisotropy". Applied Physics Letters 95.16 (2009). DOI: 10 . 1063 / 1.3238314. arXiv: 0909.4733 (cit. on pp. 78, 94).

[118] E. Martinez, L. Torres, and L. Lopez-Diaz. "Oscillator based on pinned domain walls driven by direct current". Physical Review B 83.17 (2011), p. 174444. DOI: $10.1103 /$ PhysRevB. 83.174444 (cit. on pp. 78, 82, 94).

[119] B. Ghosh and G. Solanki. "Domain wall oscillators". Applied Physics A: Materials Science and Processing 118.1 (2014), pp. 101-106. DOI: 10.1007 / s 00339 014-8768-6 (cit. on p. 78).

[120] D. S. Han et al. "Magnetic domain-wall motion by propagating spin waves". Applied Physics Letters 94.11 (2009). DOI: 10 .1063/1. 309840 9. arXiv: 0902. 0220 (cit. on p. 86).

[121] K. S. Lee, D. S. Han, and S. K. Kim. "Physical origin and generic control of magnonic band gaps of dipole-exchange spin waves in width-modulated nanostrip waveguides". Physical Review Letters 102.12 (2009), pp. 1-4. DOI: 10.1103/PhysRevLett.102.127202. arXiv: 0811.0411 (cit. on p. 86).

[122] S.-K. Kim. "Micromagnetic computer simulations of spin waves in nanometrescale patterned magnetic elements". Journal of Physics D: Applied Physics 43.26 (2010), p. 264004. DOI: 10 . 1088/0022-3727/43/26/264004 (cit. on p. 86).

[123] K. Wagner et al. "Magnetic domain walls as reconfigurable spin-wave nanochannels". Nature Nanotechnology February (2016), pp. 1-6. DOI: 10 . 1038 / nnano . 2015.339 (cit. on p. 86).

[124] G. Consolo et al. "Boundary conditions for spin-wave absorption based on different site-dependent damping functions". IEEE Transactions on Magnetics 43.6 (2007), pp. 2974-2976. DOI: 10 . 1109 / TMAG . 2007 . 893124 (cit. on p. 93).

[125] T. Koyama et al. "Observation of the intrinsic pinning of a magnetic domain wall in a ferromagnetic nanowire". Nature Materials 10.3 (2011), pp. 194-197. DOI: $10.1038 /$ nmat 2961 (cit. on p. 94).

[126] S. Moretti, V. Raposo, and E. Martinez. "Influence of Joule heating on currentinduced domain wall depinning". Journal of Applied Physics 119.21 (2016). DOI: $10.1063 / 1.4953008$. arXiv: 1604.00522 (cit. on p. 95). 
[127] T. Brächer et al. "Creation of unidirectional spin-wave emitters by utilizing interfacial Dzyaloshinskii-Moriya interaction". Physical Review B 95.6 (2017), p. 064429. DOI: 10.1103 /PhysRevB.95.064429. arXiv: 1611.07841 (cit. on p. 101).

[128] J. V. Kim, R. L. Stamps, and R. E. Camley. "Spin Wave Power Flow and Caustics in Ultrathin Ferromagnets with the Dzyaloshinskii-Moriya Interaction". Physical Review Letters 117.19 (2016), pp. 1-5. DOI: 10 . 1103 / PhysRevLett. 117.197204. arXiv: 1606.08159 (cit. on p. 101).

[129] W. Wang et al. "Magnon-driven domain-wall motion with the DzyaloshinskiiMoriya interaction". Physical Review Letters 114.8 (2015), pp. 1-5. DOI: 10 . 1103/PhysRevLett.114.087203. arXiv: 1406.5997 (cit. on p. 101).

[130] S. M. Seo et al. "Current-induced control of spin-wave attenuation". Physical Review Letters 102.14 (2009), pp. 100-103. DOI: 10 . 1103 / PhysRevLett . 102.147202 (cit. on p. 105).

[131] A. L. Balk et al. "Simultaneous control of the Dzyaloshinskii-Moriya interaction and magnetic anisotropy in nanomagnetic trilayers". Physical Review Letters 119.7 (2017), p. 077205. DOI: 10.1103 / PhysRevLett. 119.077205 (cit. on p. 108).

[132] M. A. W. Schoen et al. "Ultra-low magnetic damping of a metallic ferromagnet". Nature Physics 12.9 (2016), pp. 839-842. DOI: 10 . 1038 / nphys3770. arXiv: 1512.03610 (cit. on p. 111). 

Come ogni fior languisce e giovinezza cede a vecchiaia, anche la vita in tutti $i$ gradi suoi fiorisce, insieme ad ogni senno e virtù, né può durare eterna. Quando la vita chiama, il cuore sia pronto a partire ed a ricominciare, per offrirsi sereno e valoroso ad altri, nuovi vincoli e legami. Ogni inizio contiene una magia che ci protegge e a vivere ci aiuta.

Dobbiamo attraversare spazi e spazi senza fermare in alcun d'essi il piede, lo spirto universal non vuol legarci ma su di grado in grado sollevarci. Appena ci avvezziamo ad una sede rischiamo di infiacchire nell'ignavia; sol chi è disposto a muoversi e partire vince la consuetudine inceppante.

Forse il momento stesso della morte ci farà andare incontro a nuovi spazi; della vita il richiamo non ha fine... Su, cuore mio, congedati e guarisci! 